\title{
Unraveling the way acetaldehyde is formed from acetylene: a study based on DFT
}

Uzma Habib*, Mahum Riaz ${ }^{\ddagger}$ and Matthias Hofmann ${ }^{\S}$

* Research Center for Modeling \& Simulation (RCMS), National University of Sciences \& Technology (NUST), Islamabad, Pakistan.

§Anorganisch Chemisches Institüt, Ruprecht-Karls-Universität, Heidelberg, Germany

\section{Figures}

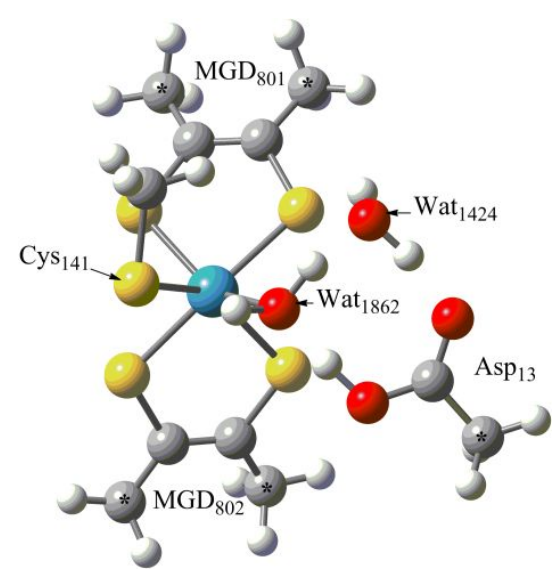

Figure S-1: Optimized reduced active site small model of AH.

Atoms labeled $(*)$ were kept fixed at their X-ray crystal structure positions.

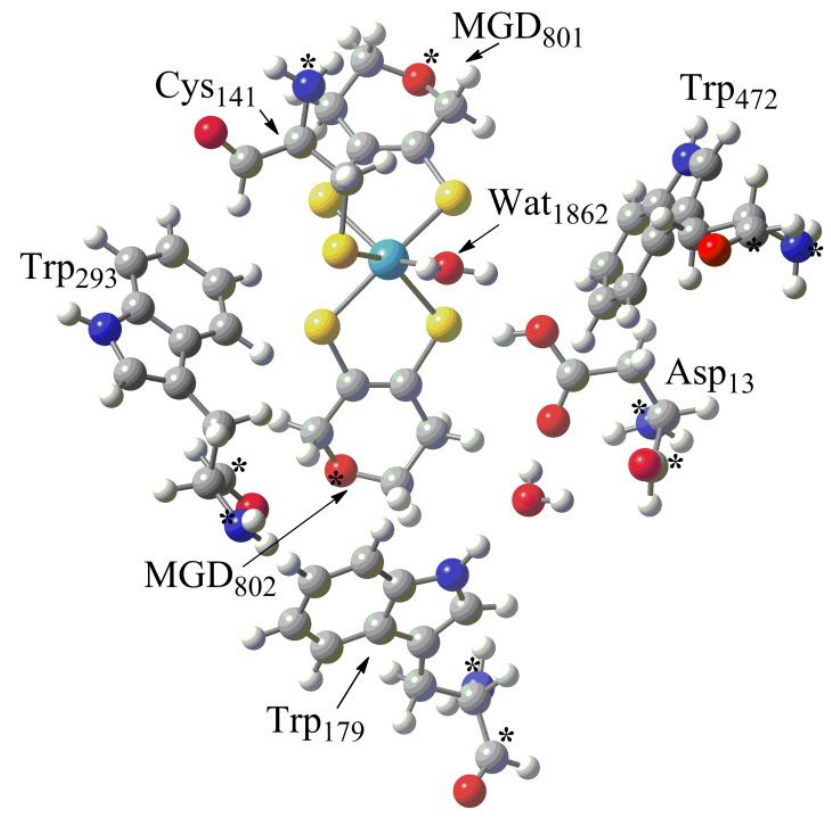

Figure S-2: Optimized reduced active site large model of AH.

Atoms labeled $(*)$ were kept fixed at their X-ray crystal structure positions. 


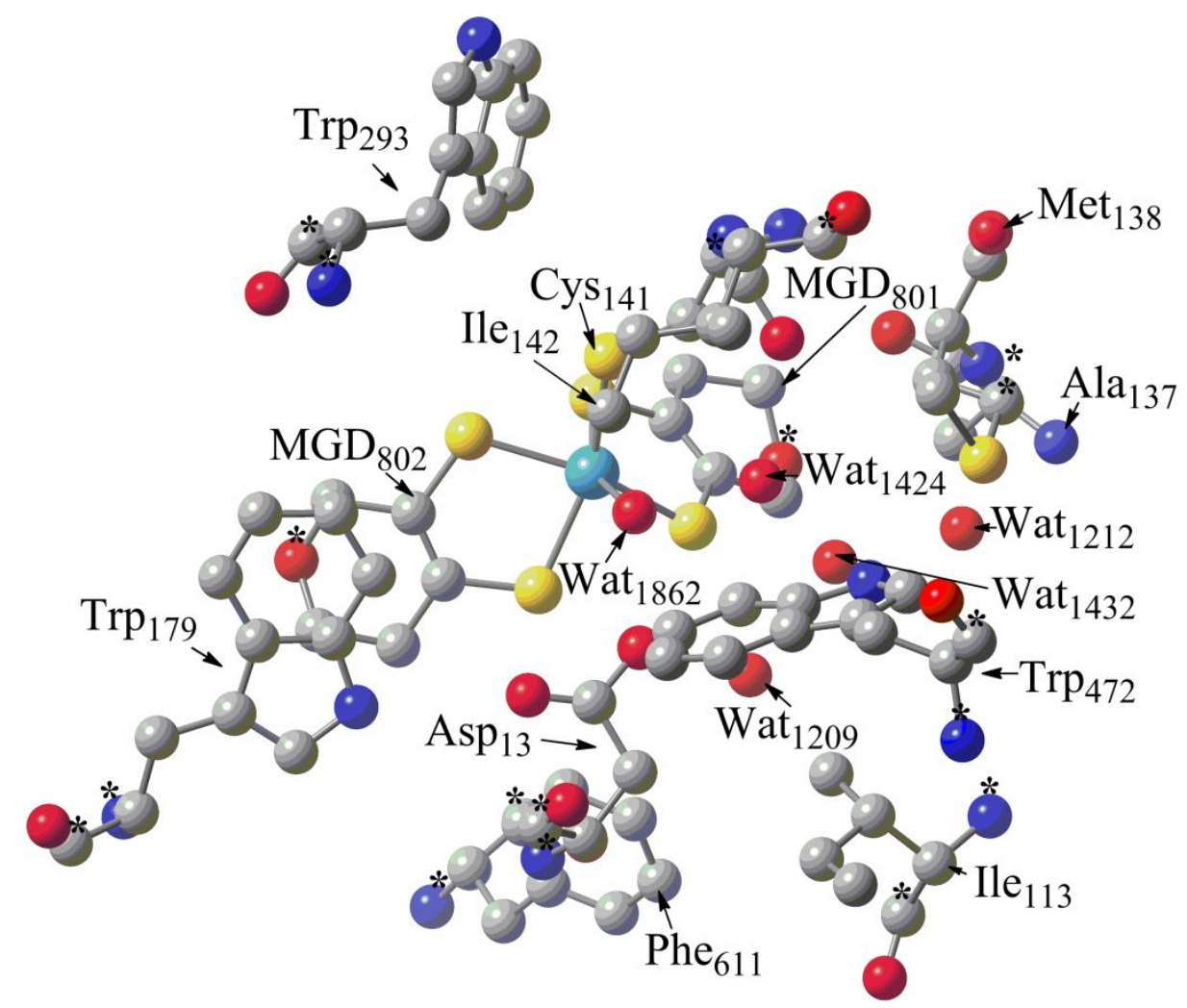

Figure S-3: Optimized reduced active site large model (water containing) complex of AH.

Atoms labeled $\left(^{*}\right)$ were kept fixed at their X-ray crystal structure positions. Hydrogen atoms were excluded to get the clear view of the selected active site model.

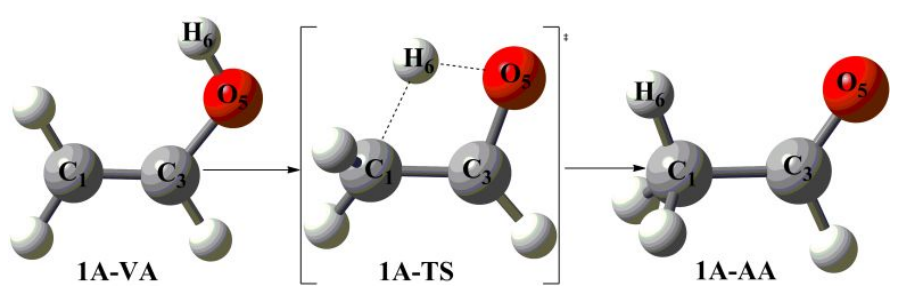

Figure S-4: Optimized geometries for the intramolecular tautomerization of vinyl alcohol to acetaldehyde.

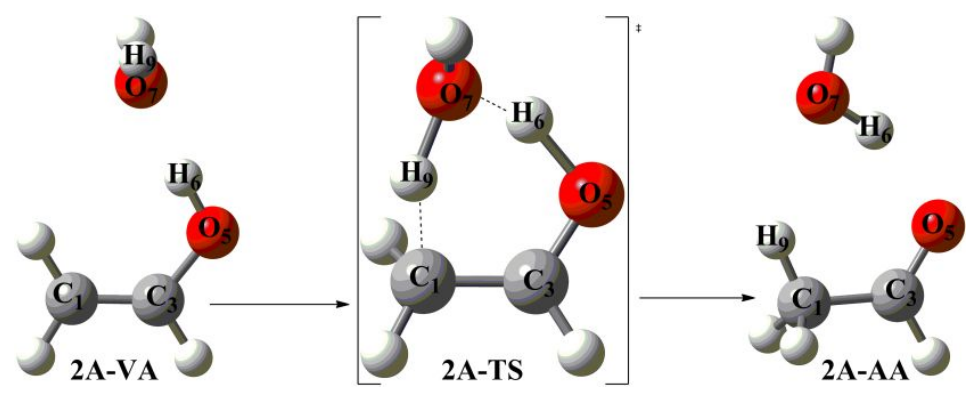

Figure S-5: Optimized geometries for the single water molecule catalyzed, intermolecular tautomerization of vinyl alcohol to acetaldehyde. 


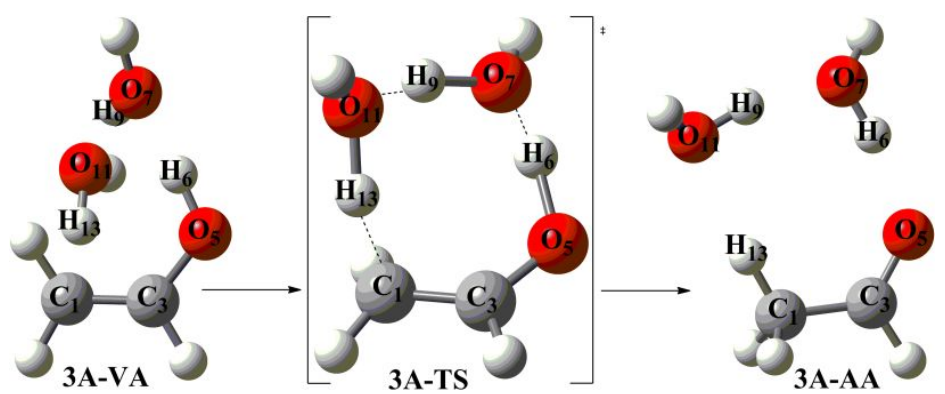

Figure S-6: Optimized geometries for the intermolecular tautomerization of vinyl alcohol to acetaldehyde catalyzed by two water molecules.

\section{Characterization of transition structures}

Contribution of relevant negative eigenvalue followed in the optimization procedure for model complexes.

\begin{tabular}{|c|c|}
\hline & Eigenvalue \\
\hline SE-TS & -0.01325 \\
\hline SN-TS & -0.10263 \\
\hline LE-TS & -0.01978 \\
\hline LN-TS & -0.02349 \\
\hline XE-TS & -0.02178 \\
\hline XN-TS & -0.03256 \\
\hline MoN-TS & -0.01845 \\
\hline
\end{tabular}

Cartesian coordinates of structures optimized at B3LYP/Lanl2DZ* and electronic energies ( $E$ in a.u.) from single energy point calculations at B3LYP/SDDp

SE-E, $E=-852.6729833$

$\begin{array}{llll}\mathrm{C} & -5.23658240 & -1.91402715 & -0.91500007 \\ \mathrm{C} & 2.88226722 & -2.54888919 & -2.74626821 \\ \mathrm{C} & 4.65402835 & -0.36371603 & -1.91262315 \\ \mathrm{C} & -1.25242709 & 4.81533937 & -0.13309501 \\ \mathrm{C} & -2.38366518 & 3.26073125 & -2.30836617 \\ \mathrm{C} & -3.93093730 & -2.15194916 & -0.17802701 \\ \mathrm{O} & -3.52630727 & -1.11604908 & 0.56556604 \\ \mathrm{O} & -3.29921025 & -3.26379025 & -0.31892002 \\ \mathrm{C} & 1.95872315 & -1.38274210 & 3.07485023 \\ \mathrm{~S} & 0.78952206 & 0.13132001 & 2.96817622 \\ \mathrm{C} & 2.47808719 & -1.57391612 & -1.67924413\end{array}$




\begin{tabular}{lccc}
$\mathrm{S}$ & 0.73457106 & -1.62909213 & -1.12419509 \\
$\mathrm{C}$ & 3.29158025 & -0.56227104 & -1.27119610 \\
$\mathrm{~S}$ & 2.69882421 & 0.63833005 & 0.01331900 \\
$\mathrm{C}$ & -1.00361107 & 3.33218425 & -0.22617602 \\
$\mathrm{~S}$ & 0.22890802 & 2.57378020 & 0.88981107 \\
$\mathrm{C}$ & -1.58594812 & 2.58849820 & -1.21186509 \\
$\mathrm{~S}$ & -1.34290910 & 0.77830606 & -1.26477910 \\
$\mathrm{~W}$ & 0.36241003 & 0.22698702 & 0.38307703 \\
$\mathrm{H}$ & 2.13991216 & -3.36135126 & -2.85405322 \\
$\mathrm{H}$ & 2.97258823 & -2.05693616 & -3.73886329 \\
$\mathrm{H}$ & 3.86557830 & -3.01377223 & -2.52686719 \\
$\mathrm{H}$ & 4.55068335 & -0.14937201 & -2.99637323 \\
$\mathrm{H}$ & 5.18986840 & 0.48635104 & -1.45110911 \\
$\mathrm{H}$ & 5.29517939 & -1.26422810 & -1.81271914 \\
$\mathrm{H}$ & -3.25334125 & 3.82180229 & -1.90601215 \\
$\mathrm{H}$ & -1.75574213 & 3.98623530 & -2.86591622 \\
$\mathrm{H}$ & -2.76809321 & 2.52042419 & -3.03404423 \\
$\mathrm{H}$ & -2.33159518 & 5.03727039 & -0.00075900 \\
$\mathrm{H}$ & -0.70967805 & 5.25837840 & 0.72231005 \\
$\mathrm{H}$ & -0.92029707 & 5.35027341 & -1.04877408 \\
$\mathrm{H}$ & 1.57756712 & -2.21941917 & 2.46489619 \\
$\mathrm{H}$ & 2.94590822 & -1.07668608 & 2.69413421 \\
$\mathrm{H}$ & 2.02423015 & -1.68519613 & 4.13574731 \\
$\mathrm{H}$ & -5.02248238 & -1.28160410 & -1.79659114 \\
$\mathrm{H}$ & -5.66724841 & -2.87031022 & -1.24877209 \\
$\mathrm{H}$ & -5.94462846 & -1.36306910 & -0.27406202 \\
$\mathrm{O}$ & -1.15468909 & -1.17307309 & 1.34745210 \\
$\mathrm{H}$ & -2.48028219 & -1.08314008 & 0.93370107 \\
$\mathrm{H}$ & -0.96356307 & -2.27675417 & 1.12567309 \\
$\mathrm{H}$ & -0.88599707 & -0.91141607 & 2.31845418 \\
$\mathrm{O}$ & -0.95634907 & -3.50621127 & 0.65102105 \\
$\mathrm{H}$ & -1.91061814 & -3.56537827 & 0.22686102 \\
$\mathrm{H}$ & -0.24562402 & -3.48389826 & -0.05074900 \\
& & & \\
\hline & & &
\end{tabular}

SE-ES, $E=-929.9958319$

$\begin{array}{lrrr}\mathrm{C} & 1.86417200 & 5.03817300 & 1.40529800 \\ \mathrm{C} & 1.21467800 & -3.07048900 & 3.27613900 \\ \mathrm{C} & -0.90394500 & -4.61840000 & 1.96294200 \\ \mathrm{C} & -4.90309800 & 1.76529400 & -0.88849300 \\ \mathrm{C} & -3.76230700 & 2.76474300 & 1.58698800 \\ \mathrm{C} & 2.10650300 & 3.69267300 & 0.74965700 \\ \mathrm{O} & 1.25205300 & 3.40921600 & -0.24460700 \\ \mathrm{O} & 3.04312700 & 2.92059500 & 1.16739500 \\ \mathrm{C} & 1.44814800 & -2.15276900 & -2.66642200 \\ \mathrm{~S} & 0.11352100 & -0.79456700 & -2.87886500 \\ \mathrm{C} & 0.54770700 & -2.58391800 & 2.02323800 \\ \mathrm{~S} & 0.90390200 & -0.86379500 & 1.50836300 \\ \mathrm{C} & -0.42703600 & -3.29390000 & 1.39333700 \\ \mathrm{~S} & -1.24074100 & -2.60070100 & -0.12400900 \\ \mathrm{C} & -3.51251300 & 1.36585100 & -0.46898100 \\ & & & \mathrm{~S} 4\end{array}$




$\begin{array}{lccc}\mathrm{S} & -2.66104200 & 0.04045000 & -1.39319000 \\ \mathrm{C} & -2.94863800 & 1.88740800 & 0.65979800 \\ \mathrm{~S} & -1.22899600 & 1.46110600 & 1.10745100 \\ \mathrm{~W} & -0.51394100 & -0.33041300 & -0.37642200 \\ \mathrm{H} & 2.05705800 & -2.41613000 & 3.56705800 \\ \mathrm{H} & 0.50876100 & -3.09350900 & 4.13421300 \\ \mathrm{H} & 1.61030300 & -4.09994300 & 3.15660300 \\ \mathrm{H} & -1.33176900 & -4.47857900 & 2.97691800 \\ \mathrm{H} & -1.68845000 & -5.06587800 & 1.32506300 \\ \mathrm{H} & -0.07721400 & -5.35359200 & 2.05045100 \\ \mathrm{H} & -4.12971800 & 3.68035300 & 1.07759300 \\ \mathrm{H} & -4.65239100 & 2.22038400 & 1.96454600 \\ \mathrm{H} & -3.16596500 & 3.08318800 & 2.46177700 \\ \mathrm{H} & -4.97627300 & 2.85966000 & -1.05570500 \\ \mathrm{H} & -5.19831500 & 1.26005000 & -1.82654300 \\ \mathrm{H} & -5.66009700 & 1.50242100 & -0.11871200 \\ \mathrm{H} & 2.14746000 & -1.89558300 & -1.85296600 \\ \mathrm{H} & 0.93522300 & -3.09493800 & -2.41779100 \\ \mathrm{H} & 1.99434900 & -2.24279000 & -3.62236000 \\ \mathrm{H} & 0.99884300 & 4.93697000 & 2.08646000 \\ \mathrm{H} & 2.74870800 & 5.34567500 & 1.98306300 \\ \mathrm{H} & 1.60423000 & 5.79458400 & 0.64648300 \\ \mathrm{O} & 1.25265100 & 1.00396100 & -1.00104800 \\ \mathrm{C} & 7.25537400 & -0.37878700 & -1.22440300 \\ \mathrm{C} & 6.11702000 & -0.11393000 & -0.82333500 \\ \mathrm{H} & 1.19997800 & 2.38290200 & -0.60607200 \\ \mathrm{H} & 2.20180400 & 0.70496600 & -0.56265600 \\ \mathrm{H} & 8.24375800 & -0.61155100 & -1.57831800 \\ \mathrm{H} & 5.10817100 & 0.12040100 & -0.46425700 \\ \mathrm{H} & 1.17362100 & 0.71640800 & -2.00721100 \\ \mathrm{O} & 3.35931300 & 0.55621800 & 0.26452400 \\ \mathrm{H} & 3.34509700 & 1.50828900 & 0.70085100 \\ \mathrm{H} & 3.01465200 & -0.12469800 & 0.91603600\end{array}$

SE-TS, $E=-929.947802$

$\begin{array}{llcc}\mathrm{C} & -3.31969700 & 4.40011700 & -0.83160100 \\ \mathrm{C} & -3.15998800 & 2.98514300 & -0.30537300 \\ \mathrm{O} & -2.20281700 & 2.87623600 & 0.63655800 \\ \mathrm{O} & -3.86547000 & 2.02948800 & -0.75888800 \\ \mathrm{C} & -0.31092300 & -2.36156200 & 3.01184700 \\ \mathrm{~S} & 0.59791300 & -0.67631900 & 2.94457300 \\ \mathrm{C} & -1.13000100 & -3.37270700 & -2.94364100 \\ \mathrm{C} & -0.36775100 & -2.70555100 & -1.83550600 \\ \mathrm{~S} & -1.01840000 & -1.11597100 & -1.21324500 \\ \mathrm{C} & 0.84703700 & -3.15079100 & -1.41350100 \\ \mathrm{~S} & 1.73928700 & -2.25217100 & -0.05673800 \\ \mathrm{C} & 1.50562700 & -4.33819000 & -2.08901300 \\ \mathrm{C} & 4.34041100 & 2.88699800 & 0.06415200 \\ \mathrm{C} & 3.03327500 & 2.15362800 & -0.09321600 \\ \mathrm{~S} & 2.71838200 & 0.68952800 & 0.94775200\end{array}$




\begin{tabular}{lrrr}
$\mathrm{C}$ & 2.15176800 & 2.50141000 & -1.07664200 \\
$\mathrm{~S}$ & 0.52268000 & 1.67312200 & -1.17590900 \\
$\mathrm{C}$ & 2.54556100 & 3.51925100 & -2.12835300 \\
$\mathrm{~W}$ & 0.55742400 & -0.21205000 & 0.36160500 \\
$\mathrm{H}$ & -2.14896000 & -2.95539600 & -3.04343900 \\
$\mathrm{H}$ & -0.62614000 & -3.24043400 & -3.92532200 \\
$\mathrm{H}$ & -1.22547200 & -4.46472200 & -2.77432800 \\
$\mathrm{H}$ & 1.68175900 & -4.13192200 & -3.16477000 \\
$\mathrm{H}$ & 2.48346900 & -4.56768900 & -1.62628000 \\
$\mathrm{H}$ & 0.87869500 & -5.25210800 & -2.02595600 \\
$\mathrm{H}$ & 2.78981500 & 4.50587800 & -1.68157000 \\
$\mathrm{H}$ & 3.44214100 & 3.18036500 & -2.68713400 \\
$\mathrm{H}$ & 1.73099400 & 3.67377600 & -2.85985500 \\
$\mathrm{H}$ & 4.17682300 & 3.96856100 & 0.24982000 \\
$\mathrm{H}$ & 4.92512800 & 2.48330000 & 0.91116200 \\
$\mathrm{H}$ & 4.97408300 & 2.80505500 & -0.84490700 \\
$\mathrm{H}$ & -1.17034700 & -2.37194500 & 2.31989600 \\
$\mathrm{H}$ & 0.40497300 & -3.13826600 & 2.70056800 \\
$\mathrm{H}$ & -0.64598000 & -2.53232800 & 4.05138400 \\
$\mathrm{H}$ & -2.50022300 & 4.59560200 & -1.54802400 \\
$\mathrm{H}$ & -4.28610600 & 4.50655200 & -1.34768000 \\
$\mathrm{H}$ & -3.22716900 & 5.13149900 & -0.01153200 \\
$\mathrm{O}$ & -1.39462100 & 0.57117500 & 1.33303400 \\
$\mathrm{C}$ & -3.55815100 & -0.96569000 & 1.84011900 \\
$\mathrm{C}$ & -4.49691100 & -1.22273500 & 1.02514100 \\
$\mathrm{H}$ & -1.86792400 & 1.89552800 & 0.90422800 \\
$\mathrm{H}$ & -2.25840200 & -0.08278000 & 1.33333600 \\
$\mathrm{H}$ & -3.30737100 & -1.12078400 & 2.88823000 \\
$\mathrm{H}$ & -5.41301400 & -1.57584400 & 0.57734900 \\
$\mathrm{H}$ & -0.97399000 & 0.45075700 & 2.29150800 \\
$\mathrm{O}$ & -4.18321500 & -0.56050200 & -0.95402800 \\
$\mathrm{H}$ & -4.09392000 & 0.45168100 & -0.84863900 \\
$\mathrm{H}$ & -3.26262800 & -0.92080100 & -1.13963100 \\
& & & \\
\hline
\end{tabular}

SE-EP1, $E=-930.0403595$

$\begin{array}{lrrr}\mathrm{C} & -2.67465400 & 4.83927800 & 0.29386600 \\ \mathrm{C} & -2.18204500 & -3.06402200 & -2.34573000 \\ \mathrm{C} & 0.43258200 & -4.39058300 & -2.23152300 \\ \mathrm{C} & 4.67528900 & 2.32545100 & -0.90119200 \\ \mathrm{C} & 2.46374500 & 3.26496200 & -2.53051600 \\ \mathrm{C} & -2.92590900 & 3.34681200 & 0.40470100 \\ \mathrm{O} & -2.34314600 & 2.61303700 & 1.25687900 \\ \mathrm{O} & -3.81321400 & 2.88634000 & -0.51669800 \\ \mathrm{C} & 0.13118500 & -2.41527000 & 3.10164200 \\ \mathrm{~S} & 1.34459700 & -0.95130500 & 2.87521100 \\ \mathrm{C} & -1.08355100 & -2.56020500 & -1.45530500 \\ \mathrm{~S} & -1.32695200 & -0.94972400 & -0.62004500 \\ \mathrm{C} & 0.13888000 & -3.15715900 & -1.39357400 \\ \mathrm{~S} & 1.48657400 & -2.42556700 & -0.35505200 \\ \mathrm{C} & 3.28525200 & 1.78310400 & -0.69329500\end{array}$




$\begin{array}{lccc}\mathrm{S} & 3.06979800 & 0.33404300 & 0.41030800 \\ \mathrm{C} & 2.23344300 & 2.26598900 & -1.41439400 \\ \mathrm{~S} & 0.53715800 & 1.65333800 & -1.08927600 \\ \mathrm{~W} & 0.74586900 & -0.25777100 & 0.42316500 \\ \mathrm{H} & -3.16650400 & -2.64135600 & -2.06846100 \\ \mathrm{H} & -2.00325300 & -2.79178700 & -3.40838000 \\ \mathrm{H} & -2.26786900 & -4.16804700 & -2.30350000 \\ \mathrm{H} & 0.32248700 & -4.16898900 & -3.31267700 \\ \mathrm{H} & 1.46785800 & -4.74064700 & -2.06383900 \\ \mathrm{H} & -0.25153600 & -5.23148900 & -1.99252300 \\ \mathrm{H} & 2.92360800 & 4.20585100 & -2.16018500 \\ \mathrm{H} & 3.14747200 & 2.84754100 & -3.29838500 \\ \mathrm{H} & 1.51457000 & 3.52915200 & -3.03252600 \\ \mathrm{H} & 4.71175700 & 3.42287800 & -0.74051700 \\ \mathrm{H} & 5.39435700 & 1.85778500 & -0.20322400 \\ \mathrm{H} & 5.04616800 & 2.13590200 & -1.93195400 \\ \mathrm{H} & -0.86143000 & -2.15810500 & 2.69530500 \\ \mathrm{H} & 0.53330200 & -3.28440800 & 2.55687600 \\ \mathrm{H} & 0.05851700 & -2.63130900 & 4.18352600 \\ \mathrm{H} & -1.81028400 & 4.98748300 & -0.38061100 \\ \mathrm{H} & -3.54733400 & 5.35692400 & -0.13401400 \\ \mathrm{H} & -2.41220400 & 5.25105600 & 1.28127600 \\ \mathrm{O} & -0.53010000 & 0.78416200 & 1.75423500 \\ \mathrm{C} & -5.12412500 & -1.60214700 & 0.38822600 \\ \mathrm{C} & -5.16338600 & -0.34287200 & -0.11681300 \\ \mathrm{H} & -1.24106400 & 1.47677300 & 1.56586600 \\ \mathrm{H} & -4.19602700 & -2.17952400 & 0.44852200 \\ \mathrm{H} & -6.04381800 & -2.05785600 & 0.76669400 \\ \mathrm{H} & -6.08012000 & 0.25607800 & -0.16490300 \\ \mathrm{H} & -0.21928400 & 0.60204800 & 2.70303000 \\ \mathrm{O} & -4.07948300 & 0.36511600 & -0.65187400 \\ \mathrm{H} & -3.92365700 & 1.83543800 & -0.54407100 \\ \mathrm{H} & -3.18151000 & -0.15815400 & -0.69080200 \\ & & & \\ & & & \end{array}$

SE-EP2, $E=-930.0403595$

$\begin{array}{llcc}\mathrm{C} & -1.89699900 & 5.25175100 & -0.47093400 \\ \mathrm{C} & -2.30262800 & -2.75557200 & -2.79268600 \\ \mathrm{C} & -0.01435800 & -4.48402000 & -2.17189900 \\ \mathrm{C} & 5.01009600 & 1.50879500 & -0.24408300 \\ \mathrm{C} & 3.31361400 & 2.70882900 & -2.27143900 \\ \mathrm{C} & -2.30738000 & 3.87284300 & 0.01814000 \\ \mathrm{O} & -1.66677500 & 3.28794500 & 0.95281100 \\ \mathrm{O} & -3.36128800 & 3.34539800 & -0.63258000 \\ \mathrm{C} & -0.86056600 & -2.22667800 & 3.00705100 \\ \mathrm{~S} & 0.62087400 & -1.02072500 & 2.92574000 \\ \mathrm{C} & -1.29210900 & -2.36930500 & -1.74872900 \\ \mathrm{~S} & -1.36196300 & -0.64846600 & -1.11438900 \\ \mathrm{C} & -0.23925000 & -3.17483800 & -1.43206600 \\ \mathrm{~S} & 1.01211600 & -2.62217400 & -0.18222400\end{array}$




\begin{tabular}{lrrr}
$\mathrm{C}$ & 3.53676500 & 1.21127400 & -0.28304200 \\
$\mathrm{~S}$ & 2.89339600 & -0.13007800 & 0.78696400 \\
$\mathrm{C}$ & 2.72436600 & 1.83110900 & -1.18536500 \\
$\mathrm{~S}$ & 0.91753600 & 1.54045900 & -1.14148800 \\
$\mathrm{~W}$ & 0.54065600 & -0.32838500 & 0.38662000 \\
$\mathrm{H}$ & -3.12386700 & -2.01765700 & -2.85117100 \\
$\mathrm{H}$ & -1.84585000 & -2.81368500 & -3.80426200 \\
$\mathrm{H}$ & -2.75042800 & -3.74974100 & -2.58521600 \\
$\mathrm{H}$ & 0.21795300 & -4.29418300 & -3.24039200 \\
$\mathrm{H}$ & 0.83446000 & -5.04353800 & -1.73750400 \\
$\mathrm{H}$ & -0.90959900 & -5.13884100 & -2.13950500 \\
$\mathrm{H}$ & 3.87973100 & 3.56763900 & -1.85263600 \\
$\mathrm{H}$ & 4.01728700 & 2.13074600 & -2.90535400 \\
$\mathrm{H}$ & 2.52301100 & 3.11514100 & -2.92910000 \\
$\mathrm{H}$ & 5.20268600 & 2.58977700 & -0.08295400 \\
$\mathrm{H}$ & 5.50897200 & 0.95236600 & 0.57114000 \\
$\mathrm{H}$ & 5.51771000 & 1.22731700 & -1.19231800 \\
$\mathrm{H}$ & -1.74615700 & -1.79573400 & 2.50667800 \\
$\mathrm{H}$ & -0.57355500 & -3.15954500 & 2.49568200 \\
$\mathrm{H}$ & -1.08642400 & -2.41890200 & 4.07215600 \\
$\mathrm{H}$ & -1.06214100 & 5.12145100 & -1.18488700 \\
$\mathrm{H}$ & -2.73110500 & 5.75543300 & -0.98319900 \\
$\mathrm{H}$ & -1.52729600 & 5.85347100 & 0.37503900 \\
$\mathrm{O}$ & -0.75823700 & 0.95641100 & 1.50383800 \\
$\mathrm{C}$ & -4.34288300 & -1.27207000 & 0.58138600 \\
$\mathrm{C}$ & -3.66126500 & 0.07266000 & 0.46802000 \\
$\mathrm{H}$ & -1.09837100 & 1.89520200 & 1.26034300 \\
$\mathrm{H}$ & -5.11458800 & -1.38596200 & -0.19763800 \\
$\mathrm{H}$ & -3.59855300 & -2.08240700 & 0.50808500 \\
$\mathrm{H}$ & -2.74264800 & 0.24593000 & 1.06156900 \\
$\mathrm{H}$ & -0.53373500 & 0.75266100 & 2.47941600 \\
$\mathrm{O}$ & -4.22559800 & 1.04159200 & -0.16189400 \\
$\mathrm{H}$ & -3.65892700 & 2.33553200 & -0.36943300 \\
$\mathrm{H}$ & -4.82766000 & -1.34561800 & 1.57840100 \\
& & & \\
\hline & & & \\
& & &
\end{tabular}

$\begin{array}{lrrr}\mathbf{S N}-\mathbf{E}, E= & -775.589761 & & \\ \mathrm{C} & -6.48150700 & -1.72750600 & -0.93201400 \\ \mathrm{C} & 3.04119700 & -3.36208000 & -1.97111000 \\ \mathrm{C} & 4.93442600 & -1.17126700 & -1.51293700 \\ \mathrm{C} & -0.67062100 & 4.60584500 & -0.98309300 \\ \mathrm{C} & -1.85271400 & 2.72382900 & -2.85095600 \\ \mathrm{C} & -5.34597900 & -1.61477700 & 0.18566400 \\ \mathrm{O} & -4.11251400 & -1.40107500 & -0.22369300 \\ \mathrm{O} & -5.76593600 & -1.74267000 & 1.40536900 \\ \mathrm{C} & 1.69356400 & 1.11596100 & 3.56054800 \\ \mathrm{~S} & 0.50491300 & -0.20146700 & 2.87001200 \\ \mathrm{C} & 2.65194000 & -2.19036200 & -1.12508900 \\ \mathrm{~S} & 0.87485800 & -2.12171100 & -0.69873100 \\ \mathrm{C} & 3.51680200 & -1.15792000 & -0.88929500\end{array}$




$\begin{array}{lccc}\mathrm{S} & 2.89345400 & 0.27462800 & 0.11388100 \\ \mathrm{C} & -0.50527700 & 3.10552000 & -0.79136700 \\ \mathrm{~S} & 0.49345200 & 2.40830100 & 0.59644400 \\ \mathrm{C} & -1.03994800 & 2.23679800 & -1.69633500 \\ \mathrm{~S} & -0.86248000 & 0.43795200 & -1.57818700 \\ \mathrm{~W} & 0.42354100 & -0.02196700 & 0.41078200 \\ \mathrm{H} & 2.33008300 & -4.20037500 & -1.84242700 \\ \mathrm{H} & 3.04811800 & -3.11217500 & -3.05687800 \\ \mathrm{H} & 4.05668700 & -3.73692000 & -1.72168900 \\ \mathrm{H} & 4.87175000 & -1.23749100 & -2.61889600 \\ \mathrm{H} & 5.47592400 & -0.24186600 & -1.25269900 \\ \mathrm{H} & 5.53632600 & -2.03386600 & -1.15453000 \\ \mathrm{H} & -2.76737000 & 3.26253500 & -2.51795000 \\ \mathrm{H} & -1.28447600 & 3.43145600 & -3.49574200 \\ \mathrm{H} & -2.18396700 & 1.88186000 & -3.48635800 \\ \mathrm{H} & -1.74319300 & 4.89611100 & -0.98810100 \\ \mathrm{H} & -0.17225400 & 5.16520600 & -0.16764700 \\ \mathrm{H} & -0.23280000 & 4.95059900 & -1.94566000 \\ \mathrm{H} & 2.67720600 & 0.96817700 & 3.08199200 \\ \mathrm{H} & 1.30332700 & 2.11468900 & 3.29870300 \\ \mathrm{H} & 1.76003900 & 0.99388100 & 4.65854000 \\ \mathrm{H} & -7.00936800 & -2.69219200 & -0.80312000 \\ \mathrm{H} & -7.21251100 & -0.91157700 & -0.76973000 \\ \mathrm{H} & -6.05227800 & -1.65536600 & -1.94732000 \\ \mathrm{O} & -1.57465400 & -0.91309200 & 0.96449800 \\ \mathrm{H} & -1.79908400 & -0.81706300 & 1.92231200 \\ \mathrm{H} & -2.43782300 & -1.09283600 & 0.42114300\end{array}$

$\begin{array}{lccc}\text { SN-ES, } E=-852.9095091 & \\ \mathrm{C} & -6.20648700 & -1.48584500 & -2.05468500 \\ \mathrm{C} & 3.32498800 & -3.37794500 & -2.12085900 \\ \mathrm{C} & 5.21038000 & -1.29354200 & -1.28751600 \\ \mathrm{C} & -0.26075100 & 4.62576000 & -0.96986200 \\ \mathrm{C} & -1.26789500 & 2.92428000 & -3.09562500 \\ \mathrm{C} & -6.16366000 & -1.17685900 & -0.56296500 \\ \mathrm{O} & -4.95803400 & -1.57758200 & -0.02731800 \\ \mathrm{O} & -7.09098800 & -0.62191700 & 0.09193400 \\ \mathrm{C} & 2.18620600 & 1.05098700 & 3.43516100 \\ \mathrm{~S} & 0.82494400 & -0.13936400 & 2.83481700 \\ \mathrm{C} & 2.88769300 & -2.24755600 & -1.22092100 \\ \mathrm{~S} & 1.10697200 & -2.14408700 & -0.82823200 \\ \mathrm{C} & 3.75672100 & -1.26533600 & -0.83498600 \\ \mathrm{~S} & 3.16929900 & 0.14285900 & 0.19901200 \\ \mathrm{C} & -0.15016900 & 3.12120200 & -0.86651400 \\ \mathrm{~S} & 0.77384700 & 2.40053200 & 0.55862800 \\ \mathrm{C} & -0.63625000 & 2.31913600 & -1.85758000 \\ \mathrm{~S} & -0.50241600 & 0.49612000 & -1.74797200 \\ \mathrm{~W} & 0.70408600 & -0.02784100 & 0.36582600 \\ \mathrm{H} & 2.56545200 & -4.18334900 & -2.13894900 \\ \mathrm{H} & 3.46936500 & -3.04124000 & -3.17291100\end{array}$




$\begin{array}{lrrr}\mathrm{H} & 4.28784200 & -3.82236400 & -1.78849700 \\ \mathrm{H} & 5.28991200 & -1.24386200 & -2.39545600 \\ \mathrm{H} & 5.75902500 & -0.42675600 & -0.87128700 \\ \mathrm{H} & 5.73935800 & -2.21772400 & -0.96260600 \\ \mathrm{H} & -2.17586600 & 3.52163700 & -2.85463000 \\ \mathrm{H} & -0.56366200 & 3.60557600 & -3.62252600 \\ \mathrm{H} & -1.56785600 & 2.13157800 & -3.80756800 \\ \mathrm{H} & -1.31385200 & 4.95111400 & -1.11709300 \\ \mathrm{H} & 0.11651700 & 5.11014800 & -0.04816000 \\ \mathrm{H} & 0.32602900 & 5.03578800 & -1.82390100 \\ \mathrm{H} & 3.14266700 & 0.75774200 & 2.96513100 \\ \mathrm{H} & 1.92732400 & 2.07784200 & 3.12058400 \\ \mathrm{H} & 2.24968400 & 0.99005300 & 4.53984500 \\ \mathrm{H} & -5.91182400 & -2.53476000 & -2.23462600 \\ \mathrm{H} & -7.21550900 & -1.29250600 & -2.45182000 \\ \mathrm{H} & -5.46778300 & -0.84831600 & -2.57437000 \\ \mathrm{O} & -1.04832000 & -0.97168300 & 0.95229200 \\ \mathrm{C} & -3.26514600 & -0.70071300 & 2.44602400 \\ \mathrm{C} & -4.42849700 & -0.69249600 & 2.89347700 \\ \mathrm{H} & -4.77335500 & -1.25486500 & 0.92331600 \\ \mathrm{H} & -1.46818700 & -1.40270800 & 0.16210400 \\ \mathrm{H} & -2.21841600 & -0.75559800 & 1.92738700 \\ \mathrm{H} & -5.40558400 & -0.63113400 & 3.34070900\end{array}$

SN-TS, $E=-852.8890319$

$\begin{array}{lrrr}\mathrm{C} & -6.37696200 & -1.07294400 & -1.79153100 \\ \mathrm{C} & 3.00046000 & -3.58875100 & -2.12009200 \\ \mathrm{C} & 5.03630100 & -1.60246100 & -1.39962100 \\ \mathrm{C} & -0.03353700 & 4.64717100 & -1.06153400 \\ \mathrm{C} & -1.20990900 & 2.96539200 & -3.11484200 \\ \mathrm{C} & -6.15428800 & -1.26251700 & -0.29383000 \\ \mathrm{O} & -5.71210500 & -0.10273300 & 0.29463800 \\ \mathrm{O} & -6.32325500 & -2.35902500 & 0.31346800 \\ \mathrm{C} & 2.36967800 & 1.11586100 & 3.33073800 \\ \mathrm{~S} & 0.89884600 & 0.00322100 & 2.85433200 \\ \mathrm{C} & 2.66460500 & -2.40682900 & -1.24207600 \\ \mathrm{~S} & 0.91187500 & -2.19054500 & -0.77727300 \\ \mathrm{C} & 3.60403300 & -1.47332300 & -0.90650500 \\ \mathrm{~S} & 3.14439600 & -0.01158100 & 0.11903800 \\ \mathrm{C} & -0.02999200 & 3.14291600 & -0.91539400 \\ \mathrm{~S} & 0.88942800 & 2.40994800 & 0.49753200 \\ \mathrm{C} & -0.59835700 & 2.34814200 & -1.86867500 \\ \mathrm{~S} & -0.58437700 & 0.52214900 & -1.69432500 \\ \mathrm{~W} & 0.68911800 & -0.03106400 & 0.37808700 \\ \mathrm{H} & 2.18950500 & -4.34188100 & -2.09238600 \\ \mathrm{H} & 3.13512300 & -3.29165700 & -3.18505900 \\ \mathrm{H} & 3.94164400 & -4.08637100 & -1.80140500 \\ \mathrm{H} & 5.08507900 & -1.58606800 & -2.51026700 \\ \mathrm{H} & 5.65058600 & -0.76182300 & -1.02342100 \\ \mathrm{H} & 5.51716000 & -2.54941300 & -1.06561800 \\ & & & \mathrm{~S} 10\end{array}$




$\begin{array}{lrrr}\mathrm{H} & -2.06626300 & 3.63472500 & -2.87531900 \\ \mathrm{H} & -0.46602400 & 3.57567100 & -3.67258800 \\ \mathrm{H} & -1.58181600 & 2.17744400 & -3.79738800 \\ \mathrm{H} & -1.06112600 & 5.04376700 & -1.21341400 \\ \mathrm{H} & 0.38571400 & 5.12977300 & -0.15741500 \\ \mathrm{H} & 0.57497500 & 4.98807600 & -1.93049000 \\ \mathrm{H} & 3.28195800 & 0.72318000 & 2.84593900 \\ \mathrm{H} & 2.17994700 & 2.14203800 & 2.96818200 \\ \mathrm{H} & 2.47260300 & 1.11059200 & 4.43400500 \\ \mathrm{H} & -5.38906400 & -1.04107400 & -2.28748100 \\ \mathrm{H} & -6.96570700 & -1.91064600 & -2.19942800 \\ \mathrm{H} & -6.87642400 & -0.10860600 & -1.98986400 \\ \mathrm{O} & -1.00884600 & -0.90810400 & 1.10088300 \\ \mathrm{C} & -2.91533200 & -0.09622800 & 2.11064000 \\ \mathrm{C} & -3.94881100 & -0.81599400 & 2.26249600 \\ \mathrm{H} & -5.14351800 & -0.25896700 & 1.18127900 \\ \mathrm{H} & -1.53837200 & -1.39901600 & 0.42141500 \\ \mathrm{H} & -2.28051000 & 0.77336400 & 1.99563700 \\ \mathrm{H} & -4.29658600 & -1.83918600 & 2.37726500\end{array}$

SN-EP1, $E=-852.952681$

$\begin{array}{lccc}\mathrm{C} & -6.37696700 & -0.61491800 & -1.34402800 \\ \mathrm{C} & 2.80824900 & -3.64944500 & -2.20436300 \\ \mathrm{C} & 4.98160200 & -1.78193700 & -1.56153400 \\ \mathrm{C} & 0.29196900 & 4.71518200 & -0.87323600 \\ \mathrm{C} & -1.07314000 & 3.13639700 & -2.89119300 \\ \mathrm{C} & -7.22891400 & -0.87781900 & -0.05123600 \\ \mathrm{O} & -6.57051000 & -0.83139500 & 1.08303900 \\ \mathrm{O} & -8.50357600 & -1.08032100 & -0.21569400 \\ \mathrm{C} & 2.46285200 & 0.90229300 & 3.40686300 \\ \mathrm{~S} & 0.95527100 & -0.10145400 & 2.82381300 \\ \mathrm{C} & 2.59559300 & -2.48857800 & -1.27453800 \\ \mathrm{~S} & 0.85893100 & -2.11592900 & -0.83779800 \\ \mathrm{C} & 3.59181100 & -1.61294400 & -0.96716200 \\ \mathrm{~S} & 3.24405200 & -0.13680000 & 0.10226800 \\ \mathrm{C} & 0.24653500 & 3.21253200 & -0.76934800 \\ \mathrm{~S} & 1.22926800 & 2.37872700 & 0.53349000 \\ \mathrm{C} & -0.44921200 & 2.47565500 & -1.68130600 \\ \mathrm{~S} & -0.57620800 & 0.66279600 & -1.49610300 \\ \mathrm{~W} & 0.83631400 & -0.00627900 & 0.37684200 \\ \mathrm{H} & 2.00559300 & -4.40318400 & -2.09267900 \\ \mathrm{H} & 2.80287100 & -3.32911200 & -3.26972900 \\ \mathrm{H} & 3.77859300 & -4.15395100 & -2.01946300 \\ \mathrm{H} & 4.95299100 & -1.70758900 & -2.66889000 \\ \mathrm{H} & 5.66305800 & -0.99316200 & -1.18968900 \\ \mathrm{H} & 5.43175100 & -2.76520500 & -1.30620900 \\ \mathrm{H} & -1.79060700 & 3.93524100 & -2.60704400 \\ \mathrm{H} & -0.29784200 & 3.60497500 & -3.53457400 \\ \mathrm{H} & -1.62216200 & 2.39897300 & -3.50531000 \\ \mathrm{H} & -0.72330200 & 5.15750700 & -0.79006100\end{array}$




$\begin{array}{lrrr}\mathrm{H} & 0.91524000 & 5.15147200 & -0.06947700 \\ \mathrm{H} & 0.71494500 & 5.05470300 & -1.84408700 \\ \mathrm{H} & 3.36430400 & 0.50935000 & 2.90405200 \\ \mathrm{H} & 2.31482800 & 1.95844500 & 3.12039600 \\ \mathrm{H} & 2.54522600 & 0.80593700 & 4.50577800 \\ \mathrm{H} & -5.37699100 & -1.07912600 & -1.25892200 \\ \mathrm{H} & -6.90122800 & -0.98738800 & -2.24434600 \\ \mathrm{H} & -6.22017500 & 0.47799600 & -1.45324200 \\ \mathrm{O} & -1.06486600 & -1.10278200 & 1.11025900 \\ \mathrm{C} & -2.39883700 & -0.59283900 & 1.29409400 \\ \mathrm{C} & -3.50736200 & -1.34522900 & 1.12318100 \\ \mathrm{H} & -4.54127800 & -0.95488500 & 1.22954500 \\ \mathrm{H} & -1.06181400 & -2.02817500 & 0.74986600 \\ \mathrm{H} & -2.34780800 & 0.45784000 & 1.58262100 \\ \mathrm{H} & -3.42791500 & -2.39761200 & 0.80643600\end{array}$

SN-EP2, $E=-852.995244$

$\begin{array}{lrrr}\mathrm{C} & -6.44933650 & -0.18870961 & -1.35606142 \\ \mathrm{C} & 2.56460639 & -3.64641036 & -2.40900604 \\ \mathrm{C} & 4.83356494 & -1.92052244 & -1.70375550 \\ \mathrm{C} & 0.48504033 & 4.77128443 & -0.70946964 \\ \mathrm{C} & -0.96891743 & 3.35072402 & -2.78222785 \\ \mathrm{C} & -7.11650937 & -0.90202462 & -0.12849280 \\ \mathrm{O} & -6.28143100 & -1.35020945 & 0.77696468 \\ \mathrm{O} & -8.41370564 & -0.98241277 & -0.11476697 \\ \mathrm{C} & 2.79558829 & 0.66894297 & 3.27823208 \\ \mathrm{~S} & 1.14841158 & -0.17286883 & 2.83601711 \\ \mathrm{C} & 2.39904434 & -2.48926355 & -1.45243447 \\ \mathrm{~S} & 0.71592803 & -2.14992610 & -0.84694833 \\ \mathrm{C} & 3.44593046 & -1.67042228 & -1.13317541 \\ \mathrm{~S} & 3.19682347 & -0.23622992 & -0.02349956 \\ \mathrm{C} & 0.36416082 & 3.26600370 & -0.66797355 \\ \mathrm{~S} & 1.18548501 & 2.35415402 & 0.68148217 \\ \mathrm{C} & -0.33359800 & 2.59479807 & -1.63232947 \\ \mathrm{~S} & -0.51138585 & 0.78848976 & -1.56548101 \\ \mathrm{~W} & 0.76051891 & -0.03713244 & 0.40119582 \\ \mathrm{H} & 1.68448199 & -4.31366743 & -2.37751138 \\ \mathrm{H} & 2.67581678 & -3.29884037 & -3.45858501 \\ \mathrm{H} & 3.46185432 & -4.25240165 & -2.16847952 \\ \mathrm{H} & 4.82660542 & -1.87169719 & -2.81222149 \\ \mathrm{H} & 5.54793402 & -1.16176102 & -1.33665920 \\ \mathrm{H} & 5.22394713 & -2.92075165 & -1.42053063 \\ \mathrm{H} & -1.69346832 & 4.11062831 & -2.42316832 \\ \mathrm{H} & -0.20534897 & 3.88481675 & -3.38567234 \\ \mathrm{H} & -1.51123167 & 2.66246165 & -3.45408660 \\ \mathrm{H} & -0.50922495 & 5.26166952 & -0.65126741 \\ \mathrm{H} & 1.09238200 & 5.14360018 & 0.13545251 \\ \mathrm{H} & 0.96530195 & 5.12124509 & -1.64768177 \\ \mathrm{H} & 3.60922174 & 0.17956783 & 2.71603226 \\ \mathrm{H} & 2.74396140 & 1.73407706 & 2.99516601\end{array}$




$\begin{array}{lrrr}\mathrm{H} & 2.94739052 & 0.56987619 & 4.36832132 \\ \mathrm{H} & -5.74058804 & -0.88240581 & -1.84809003 \\ \mathrm{H} & -7.20810460 & 0.15052533 & -2.08458439 \\ \mathrm{H} & -5.85722098 & 0.67794497 & -1.00328084 \\ \mathrm{O} & -1.01941680 & -0.69120476 & 1.03370181 \\ \mathrm{C} & -2.28105892 & -0.44261715 & 1.21280954 \\ \mathrm{C} & -3.26387149 & -1.48394512 & 1.64128062 \\ \mathrm{H} & -4.23353005 & -1.40757910 & 1.08640656 \\ \mathrm{H} & -2.61907041 & 0.59145027 & 1.02090113 \\ \mathrm{H} & -2.82972159 & -2.49304238 & 1.53751336 \\ \mathrm{H} & -3.54541868 & -1.33223150 & 2.70683638\end{array}$

LE-E, $E=-3328.055130$

$\begin{array}{lrrr}\mathrm{N} & -0.25783800 & 5.62668900 & -3.99734100 \\ \mathrm{C} & -1.03734100 & 5.98643200 & -2.79937100 \\ \mathrm{C} & -0.19164600 & 6.27597200 & -1.52516300 \\ \mathrm{O} & -0.66478100 & 6.52253300 & -0.39766600 \\ \mathrm{C} & -2.08437900 & 4.90075900 & -2.45705800 \\ \mathrm{C} & -1.43380800 & 3.72624900 & -1.73796100 \\ \mathrm{O} & -0.17919900 & 3.71947600 & -1.53255600 \\ \mathrm{O} & -2.26277200 & 2.78423200 & -1.32176100 \\ \mathrm{~N} & -3.35204900 & -6.14215000 & -0.29944900 \\ \mathrm{C} & -2.23493100 & -5.32623100 & 0.13893000 \\ \mathrm{C} & -2.06229800 & -5.45802200 & 1.64196900 \\ \mathrm{O} & -2.85539800 & -6.08592900 & 2.37479200 \\ \mathrm{C} & -2.15505277 & -3.77524047 & -0.09058901 \\ \mathrm{~S} & -0.24477122 & -3.86871556 & -0.27945675 \\ \mathrm{~N} & 7.78735300 & 5.98961900 & -3.20814200 \\ \mathrm{C} & 7.50109500 & 6.14567500 & -1.76031800 \\ \mathrm{C} & 8.37729200 & 7.31030400 & -1.27664200 \\ \mathrm{O} & 9.03963200 & 7.33974500 & -0.22525400 \\ \mathrm{C} & 7.62940200 & 4.87741100 & -0.86770500 \\ \mathrm{C} & 6.35979600 & 4.39241000 & -0.19813600 \\ \mathrm{C} & 5.03721900 & 4.77263600 & -0.39904100 \\ \mathrm{C} & 6.32890800 & 3.35864200 & 0.82054800 \\ \mathrm{~N} & 4.18756500 & 4.04074700 & 0.43607100 \\ \mathrm{C} & 4.95948700 & 3.16357100 & 1.19335200 \\ \mathrm{C} & 7.33215500 & 2.57922000 & 1.44663600 \\ \mathrm{C} & 4.57649000 & 2.21310600 & 2.16253200 \\ \mathrm{C} & 6.95334700 & 1.63501400 & 2.41062300 \\ \mathrm{C} & 5.58659500 & 1.44925300 & 2.76557800 \\ \mathrm{~N} & 4.22267900 & -0.98541200 & 7.75390400 \\ \mathrm{C} & 3.86611500 & -2.20789200 & 7.04666800 \\ \mathrm{C} & 4.32989400 & -2.11618800 & 5.60095100 \\ \mathrm{O} & 4.73197800 & -1.05460800 & 5.08445400 \\ \mathrm{C} & 2.31732500 & -2.54230900 & 7.06886500 \\ \mathrm{C} & 1.96774800 & -3.91363500 & 6.54626200 \\ \mathrm{C} & 1.79029600 & -5.05328700 & 7.31719900 \\ \mathrm{C} & 1.80768800 & -4.32572700 & 5.15853400 \\ \mathrm{~N} & 1.53895500 & -6.15304500 & 6.49147700 \\ & & & \mathrm{~S} 13 \\ \mathrm{~N} & & & \end{array}$




\begin{tabular}{|c|c|c|c|}
\hline $\mathrm{C}$ & 1.55039800 & -5.73357300 & 5.15856100 \\
\hline $\mathrm{C}$ & 1.85863900 & -3.64157900 & 3.91723800 \\
\hline $\mathrm{C}$ & 1.37003600 & -6.46883200 & 3.97148200 \\
\hline $\mathrm{C}$ & 1.68335500 & -4.36575500 & 2.73110600 \\
\hline $\mathrm{C}$ & 1.44677100 & -5.76824400 & 2.75928900 \\
\hline $\mathrm{N}$ & -8.85549400 & 6.06260100 & 3.46695500 \\
\hline $\mathrm{C}$ & -9.28101300 & 4.77683900 & 4.02906000 \\
\hline $\mathrm{C}$ & -9.17020300 & 4.98620200 & 5.52768500 \\
\hline $\mathrm{O}$ & -8.63387300 & 4.20100500 & 6.33148800 \\
\hline $\mathrm{C}$ & -8.54620100 & 3.50833100 & 3.52256400 \\
\hline $\mathrm{C}$ & -8.76016200 & 3.24447300 & 2.04916400 \\
\hline $\mathrm{C}$ & -9.97428200 & 3.06903600 & 1.39963100 \\
\hline $\mathrm{C}$ & -7.72696100 & 3.09579000 & 1.03715500 \\
\hline $\mathrm{N}$ & -9.75286400 & 2.82152900 & 0.03821400 \\
\hline $\mathrm{C}$ & -8.37850400 & 2.82898800 & -0.20923300 \\
\hline $\mathrm{C}$ & -6.31078500 & 3.14774300 & 1.07180200 \\
\hline $\mathrm{C}$ & -7.66351800 & 2.61379300 & -1.40389600 \\
\hline $\mathrm{C}$ & -5.59177800 & 2.93446000 & -0.11148400 \\
\hline $\mathrm{C}$ & -6.26401100 & 2.66866600 & -1.33755800 \\
\hline $\mathrm{C}$ & -3.10741100 & -3.17534200 & -5.79265000 \\
\hline $\mathrm{O}$ & -2.90296700 & -4.11753200 & -6.89275500 \\
\hline $\mathrm{C}$ & -2.10439700 & -3.38450500 & -4.67458400 \\
\hline $\mathrm{S}$ & -1.80497600 & -1.99390600 & -3.58939500 \\
\hline $\mathrm{C}$ & -1.36800500 & -4.52178000 & -4.56808400 \\
\hline $\mathrm{S}$ & -0.04898000 & -4.62599100 & -3.35044800 \\
\hline $\mathrm{C}$ & -1.51661400 & -5.67347100 & -5.55022200 \\
\hline $\mathrm{C}$ & -2.78466200 & -5.49337000 & -6.40839400 \\
\hline $\mathrm{C}$ & 4.65625100 & -1.18570500 & -2.17150300 \\
\hline $\mathrm{O}$ & 5.14339200 & 0.08297600 & -2.71723700 \\
\hline $\mathrm{C}$ & 3.13830900 & -1.24374400 & -2.15306100 \\
\hline $\mathrm{S}$ & 2.36512300 & -2.86313600 & -2.11323600 \\
\hline $\mathrm{C}$ & 2.38718700 & -0.12069100 & -2.28712000 \\
\hline $\mathrm{S}$ & 0.61278800 & -0.28351500 & -2.49409200 \\
\hline $\mathrm{C}$ & 2.99649300 & 1.26553500 & -2.41971800 \\
\hline $\mathrm{C}$ & 4.49580500 & 1.22493500 & -2.06653500 \\
\hline $\mathrm{W}$ & 0.02415000 & -2.56547300 & -2.20751000 \\
\hline $\mathrm{H}$ & -0.62770800 & -5.71122300 & -6.19668700 \\
\hline $\mathrm{H}$ & -1.56383600 & -6.63488000 & -5.01671300 \\
\hline $\mathrm{H}$ & -3.68277600 & -5.75376800 & -5.82016100 \\
\hline $\mathrm{H}$ & -2.75427500 & -6.11744100 & -7.30768100 \\
\hline $\mathrm{H}$ & -2.98820000 & -2.18064300 & -6.23449600 \\
\hline $\mathrm{H}$ & -4.14440300 & -3.26677900 & -5.41999800 \\
\hline $\mathrm{H}$ & 5.06812600 & -1.33050700 & -1.15723600 \\
\hline $\mathrm{H}$ & 5.06326300 & -1.96379800 & -2.82596200 \\
\hline $\mathrm{H}$ & 4.63386800 & 1.15601800 & -0.97656600 \\
\hline $\mathrm{H}$ & 5.02259000 & 2.11204800 & -2.43052100 \\
\hline $\mathrm{H}$ & 2.47993500 & 1.97941500 & -1.76203800 \\
\hline $\mathrm{H}$ & 2.85899400 & 1.62323800 & -3.45133500 \\
\hline $\mathrm{H}$ & -2.84438042 & -3.23960518 & 0.57544058 \\
\hline $\mathrm{H}$ & -2.42630472 & -3.55843385 & -1.1260956 \\
\hline
\end{tabular}




\begin{tabular}{|c|c|c|c|}
\hline $\mathrm{H}$ & -4.02455100 & -6.40860700 & 0.40904900 \\
\hline $\mathrm{H}$ & -3.68467700 & -6.01928200 & -1.24526100 \\
\hline $\mathrm{H}$ & -1.29455800 & -5.64782300 & -0.33844800 \\
\hline $\mathrm{H}$ & -9.17458400 & 6.23195200 & 2.51634800 \\
\hline $\mathrm{H}$ & -7.85719400 & 6.24308600 & 3.55784600 \\
\hline $\mathrm{H}$ & -10.35822200 & 4.67899700 & 3.81594200 \\
\hline $\mathrm{H}$ & -7.47439700 & 3.62158900 & 3.73053700 \\
\hline $\mathrm{H}$ & -8.87631500 & 2.65523100 & 4.13153900 \\
\hline $\mathrm{H}$ & -10.47053300 & 2.64038600 & -0.64817700 \\
\hline $\mathrm{H}$ & -10.97843000 & 3.07999000 & 1.80088200 \\
\hline $\mathrm{H}$ & -5.78334200 & 3.33646000 & 2.00433000 \\
\hline $\mathrm{H}$ & -4.50578600 & 2.95622200 & -0.11329600 \\
\hline $\mathrm{H}$ & -5.67150000 & 2.49186100 & -2.23159300 \\
\hline $\mathrm{H}$ & -8.17064100 & 2.40141300 & -2.34263800 \\
\hline $\mathrm{H}$ & 3.67680000 & -0.75429700 & 8.57446100 \\
\hline $\mathrm{H}$ & 4.47090300 & -0.20263300 & 7.15620900 \\
\hline $\mathrm{H}$ & 4.37122600 & -3.08675300 & 7.48328400 \\
\hline $\mathrm{H}$ & 1.78825400 & -1.75418000 & 6.51545800 \\
\hline $\mathrm{H}$ & 2.00408500 & -2.45898600 & 8.11924600 \\
\hline $\mathrm{H}$ & 2.02885100 & -2.56831100 & 3.87250300 \\
\hline $\mathrm{H}$ & 1.71703100 & -3.85540900 & 1.77268100 \\
\hline $\mathrm{H}$ & 1.32480900 & -6.30226800 & 1.82012800 \\
\hline $\mathrm{H}$ & 1.17347400 & -7.53835600 & 3.98701100 \\
\hline $\mathrm{H}$ & 1.35085700 & -7.09343000 & 6.80664500 \\
\hline $\mathrm{H}$ & 1.82250100 & -5.15913000 & 8.39267900 \\
\hline $\mathrm{H}$ & 5.32478900 & 0.69778900 & 3.50600700 \\
\hline $\mathrm{H}$ & 7.71046200 & 1.02363500 & 2.89730800 \\
\hline $\mathrm{H}$ & 8.38035500 & 2.70565200 & 1.18245400 \\
\hline $\mathrm{H}$ & 3.53302100 & 2.06486000 & 2.42905800 \\
\hline $\mathrm{H}$ & 3.16473300 & 4.13673600 & 0.49152700 \\
\hline $\mathrm{H}$ & 4.62457900 & 5.51049400 & -1.07338400 \\
\hline $\mathrm{H}$ & 7.03065500 & 5.56324300 & -3.73655200 \\
\hline $\mathrm{H}$ & 8.67925400 & 5.53876800 & -3.40302300 \\
\hline $\mathrm{H}$ & 6.47634500 & 6.54256000 & -1.69679000 \\
\hline $\mathrm{H}$ & 8.04608000 & 4.06727300 & -1.48659900 \\
\hline $\mathrm{H}$ & 8.37666600 & 5.08280600 & -0.08734000 \\
\hline $\mathrm{H}$ & -2.86335200 & 5.29785000 & -1.79627900 \\
\hline $\mathrm{H}$ & -2.56976600 & 4.55032600 & -3.37378100 \\
\hline $\mathrm{H}$ & 0.24607300 & 6.38265200 & -4.44868200 \\
\hline $\mathrm{H}$ & 0.27480800 & 4.76485300 & -3.91126500 \\
\hline $\mathrm{H}$ & -1.58821600 & 6.91223000 & -3.02551000 \\
\hline $\mathrm{H}$ & -9.59684100 & 5.95169700 & 5.86259800 \\
\hline $\mathrm{H}$ & 0.90254800 & 6.28865900 & -1.70798200 \\
\hline $\mathrm{H}$ & 8.38286700 & 8.16158000 & -1.98628900 \\
\hline $\mathrm{H}$ & 4.29497600 & -3.04965600 & 5.01962000 \\
\hline $\mathrm{O}$ & -1.16093287 & -1.67685182 & -0.50060315 \\
\hline $\mathrm{H}$ & -1.19555400 & -4.92574300 & 2.06655200 \\
\hline $\mathrm{H}$ & -0.97825164 & -0.76241832 & -0.20203631 \\
\hline $\mathrm{O}$ & 1.33775700 & 4.24405300 & 0.58681600 \\
\hline $\mathrm{H}$ & -1.94258224 & -2.22544449 & -0.22971366 \\
\hline
\end{tabular}




$\begin{array}{rrrr}\mathrm{H} & -1.81094500 & 1.93910200 & -0.78785500 \\ \mathrm{H} & 0.85155900 & 3.89885100 & -0.21441600 \\ \mathrm{H} & 0.92834000 & 5.09348300 & 0.85132400\end{array}$

LE-ES, $E=-3405.408248$

\begin{tabular}{|c|c|c|c|}
\hline & -0.25783800 & 5.62668900 & -3.99734100 \\
\hline $\mathrm{C}$ & -1.03734100 & 5.98643200 & -2.79937100 \\
\hline $\mathrm{C}$ & -0.19164600 & 6.27597200 & -1.52516300 \\
\hline $\mathrm{O}$ & -0.66478100 & 6.52253300 & -0.39766600 \\
\hline C & -2.08437900 & 4.90075900 & -2.45705800 \\
\hline $\mathrm{C}$ & -1.43380800 & 3.72624900 & -1.73796100 \\
\hline $\mathrm{O}$ & -0.17919900 & 3.71947600 & -1.53255600 \\
\hline $\mathrm{O}$ & -2.26277200 & 2.78423200 & -1.32176100 \\
\hline $\mathrm{N}$ & -3.35204900 & -6.14215000 & -0.29944900 \\
\hline $\mathrm{C}$ & -2.23493100 & -5.32623100 & 0.13893000 \\
\hline $\mathrm{C}$ & -2.06229800 & -5.45802200 & 1.64196900 \\
\hline $\mathrm{O}$ & -2.85539800 & -6.08592900 & 2.37479200 \\
\hline $\mathrm{C}$ & -2.40009700 & -3.80516600 & -0.21279800 \\
\hline S & -0.79864700 & -2.83857600 & -0.03114400 \\
\hline $\mathrm{N}$ & 7.78735300 & 5.98961900 & -3.20814200 \\
\hline $\mathrm{C}$ & 7.50109500 & 6.14567500 & -1.76031800 \\
\hline $\mathrm{C}$ & 8.37729200 & 7.31030400 & -1.27664200 \\
\hline $\mathrm{O}$ & 9.03963200 & 7.33974500 & -0.22525400 \\
\hline $\mathrm{C}$ & 7.62940200 & 4.87741100 & -0.86770500 \\
\hline $\mathrm{C}$ & 6.35979600 & 4.39241000 & -0.19813600 \\
\hline $\mathrm{C}$ & 5.03721900 & 4.77263600 & -0.39904100 \\
\hline $\mathrm{C}$ & 6.32890800 & 3.35864200 & 0.82054800 \\
\hline $\mathrm{N}$ & 4.18756500 & 4.04074700 & 0.43607100 \\
\hline $\mathrm{C}$ & 4.95948700 & 3.16357100 & 1.19335200 \\
\hline $\mathrm{C}$ & 7.33215500 & 2.57922000 & 1.44663600 \\
\hline $\mathrm{C}$ & 4.57649000 & 2.21310600 & 2.16253200 \\
\hline $\mathrm{C}$ & 6.95334700 & 1.63501400 & 2.41062300 \\
\hline $\mathrm{C}$ & 5.58659500 & 1.44925300 & 2.76557800 \\
\hline $\mathrm{N}$ & 4.22267900 & -0.98541200 & 7.75390400 \\
\hline $\mathrm{C}$ & 3.86611500 & -2.20789200 & 7.04666800 \\
\hline $\mathrm{C}$ & 4.32989400 & -2.11618800 & 5.60095100 \\
\hline $\mathrm{O}$ & 4.73197800 & -1.05460800 & 5.08445400 \\
\hline $\mathrm{C}$ & 2.31732500 & -2.54230900 & 7.06886500 \\
\hline $\mathrm{C}$ & 1.96774800 & -3.91363500 & 6.54626200 \\
\hline $\mathrm{C}$ & 1.79029600 & -5.05328700 & 7.31719900 \\
\hline $\mathrm{C}$ & 1.80768800 & -4.32572700 & 5.15853400 \\
\hline $\mathrm{N}$ & 1.53895500 & -6.15304500 & 6.49147700 \\
\hline $\mathrm{C}$ & 1.55039800 & -5.73357300 & 5.15856100 \\
\hline $\mathrm{C}$ & 1.85863900 & -3.64157900 & 3.91723800 \\
\hline $\mathrm{C}$ & 1.37003600 & -6.46883200 & 3.97148200 \\
\hline $\mathrm{C}$ & 1.68335500 & -4.36575500 & 2.73110600 \\
\hline $\mathrm{C}$ & 1.44677100 & -5.76824400 & 2.75928900 \\
\hline $\mathrm{N}$ & -8.85549400 & 6.06260100 & 3.46695500 \\
\hline $\mathrm{C}$ & -9.28101300 & 4.77683900 & 4.0290600 \\
\hline
\end{tabular}




\begin{tabular}{|c|c|c|c|}
\hline & -9.17020300 & 4.98620200 & 5.52768500 \\
\hline $\mathrm{O}$ & -8.63387300 & 4.20100500 & 6.33148800 \\
\hline $\mathrm{C}$ & -8.54620100 & 3.50833100 & 3.52256400 \\
\hline C & -8.76016200 & 3.24447300 & 2.04916400 \\
\hline$C$ & -9.97428200 & 3.06903600 & 1.39963100 \\
\hline & -7.72696100 & 3.09579000 & 1.03715500 \\
\hline $\mathrm{N}$ & -9.75286400 & 2.82152900 & 0.03821400 \\
\hline $\mathrm{C}$ & -8.37850400 & 2.82898800 & -0.20923300 \\
\hline $\mathrm{C}$ & -6.31078500 & 3.14774300 & 1.07180200 \\
\hline$C$ & -7.66351800 & 2.61379300 & -1.40389600 \\
\hline $\mathrm{C}$ & -5.59177800 & 2.93446000 & -0.11148400 \\
\hline $\mathrm{C}$ & -6.26401100 & 2.66866600 & -1.33755800 \\
\hline $\mathrm{C}$ & -3.10741100 & -3.17534200 & -5.79265000 \\
\hline $\mathrm{O}$ & -2.90296700 & -4.11753200 & -6.89275500 \\
\hline $\mathrm{C}$ & -2.10439700 & -3.38450500 & -4.67458400 \\
\hline $\mathrm{S}$ & -1.80497600 & -1.99390600 & -3.58939500 \\
\hline $\mathrm{C}$ & -1.36800500 & -4.52178000 & -4.56808400 \\
\hline $\mathrm{S}$ & -0.04898000 & -4.62599100 & -3.35044800 \\
\hline $\mathrm{C}$ & -1.51661400 & -5.67347100 & -5.55022200 \\
\hline $\mathrm{C}$ & -2.78466200 & -5.49337000 & -6.40839400 \\
\hline $\mathrm{C}$ & 4.65625100 & -1.18570500 & -2.17150300 \\
\hline $\mathrm{O}$ & 5.14339200 & 0.08297600 & -2.71723700 \\
\hline $\mathrm{C}$ & 3.13830900 & -1.24374400 & -2.15306100 \\
\hline $\mathrm{S}$ & 2.36512300 & -2.86313600 & -2.11323600 \\
\hline $\mathrm{C}$ & 2.38718700 & -0.12069100 & -2.28712000 \\
\hline $\mathrm{S}$ & 0.61278800 & -0.28351500 & -2.49409200 \\
\hline $\mathrm{C}$ & 2.99649300 & 1.26553500 & -2.41971800 \\
\hline $\mathrm{C}$ & 4.49580500 & 1.22493500 & -2.06653500 \\
\hline W & 0.02415000 & -2.56547300 & -2.20751000 \\
\hline $\mathrm{H}$ & -0.62770800 & -5.71122300 & -6.19668700 \\
\hline $\mathrm{H}$ & -1.56383600 & -6.63488000 & -5.01671300 \\
\hline $\mathrm{H}$ & -3.68277600 & -5.75376800 & -5.82016100 \\
\hline $\mathrm{H}$ & -2.75427500 & -6.11744100 & -7.30768100 \\
\hline $\mathrm{H}$ & -2.98820000 & -2.18064300 & -6.23449600 \\
\hline $\mathrm{H}$ & -4.14440300 & -3.26677900 & -5.41999800 \\
\hline $\mathrm{H}$ & 5.06812600 & -1.33050700 & -1.15723600 \\
\hline $\mathrm{H}$ & 5.06326300 & -1.96379800 & -2.82596200 \\
\hline $\mathrm{H}$ & 4.63386800 & 1.15601800 & -0.97656600 \\
\hline $\mathrm{H}$ & 5.02259000 & 2.11204800 & -2.43052100 \\
\hline $\mathrm{H}$ & 2.47993500 & 1.97941500 & -1.76203800 \\
\hline $\mathrm{H}$ & 2.85899400 & 1.62323800 & -3.45133500 \\
\hline $\mathrm{H}$ & -3.14993600 & -3.32801600 & 0.43198300 \\
\hline $\mathrm{H}$ & -2.72285700 & -3.71755600 & -1.25251300 \\
\hline $\mathrm{H}$ & -4.02455100 & -6.40860700 & 0.40904900 \\
\hline $\mathrm{H}$ & -3.68467700 & -6.01928200 & -1.24526100 \\
\hline $\mathrm{H}$ & -1.29455800 & -5.64782300 & -0.33844800 \\
\hline $\mathrm{H}$ & -9.17458400 & 6.23195200 & 2.51634800 \\
\hline $\mathrm{H}$ & -7.85719400 & 6.24308600 & 3.55784600 \\
\hline$H$ & -10.35822200 & 4.67899700 & 3.81594200 \\
\hline П & . & 3.62158900 & 3.73053700 \\
\hline
\end{tabular}




\begin{tabular}{|c|c|c|c|}
\hline $\mathrm{H}$ & -8.87631500 & 2.65523100 & 4.13153900 \\
\hline $\mathrm{H}$ & -10.47053300 & 2.64038600 & -0.64817700 \\
\hline $\mathrm{H}$ & -10.97843000 & 3.07999000 & 1.80088200 \\
\hline $\mathrm{H}$ & -5.78334200 & 3.33646000 & 2.00433000 \\
\hline $\mathrm{H}$ & -4.50578600 & 2.95622200 & -0.11329600 \\
\hline $\mathrm{H}$ & -5.67150000 & 2.49186100 & -2.23159300 \\
\hline $\mathrm{H}$ & -8.17064100 & 2.40141300 & -2.34263800 \\
\hline $\mathrm{H}$ & 3.67680000 & -0.75429700 & 8.57446100 \\
\hline $\mathrm{H}$ & 4.47090300 & -0.20263300 & 7.15620900 \\
\hline $\mathrm{H}$ & 4.37122600 & -3.08675300 & 7.48328400 \\
\hline $\mathrm{H}$ & 1.78825400 & -1.75418000 & 6.51545800 \\
\hline $\mathrm{H}$ & 2.00408500 & -2.45898600 & 8.11924600 \\
\hline $\mathrm{H}$ & 2.02885100 & -2.56831100 & 3.87250300 \\
\hline $\mathrm{H}$ & 1.71703100 & -3.85540900 & 1.77268100 \\
\hline $\mathrm{H}$ & 1.32480900 & -6.30226800 & 1.82012800 \\
\hline $\mathrm{H}$ & 1.17347400 & -7.53835600 & 3.98701100 \\
\hline $\mathrm{H}$ & 1.35085700 & -7.09343000 & 6.80664500 \\
\hline $\mathrm{H}$ & 1.82250100 & -5.15913000 & 8.39267900 \\
\hline $\mathrm{H}$ & 5.32478900 & 0.69778900 & 3.50600700 \\
\hline $\mathrm{H}$ & 7.71046200 & 1.02363500 & 2.89730800 \\
\hline $\mathrm{H}$ & 8.38035500 & 2.70565200 & 1.18245400 \\
\hline $\mathrm{H}$ & 3.53302100 & 2.06486000 & 2.42905800 \\
\hline $\mathrm{H}$ & 3.16473300 & 4.13673600 & 0.49152700 \\
\hline $\mathrm{H}$ & 4.62457900 & 5.51049400 & -1.07338400 \\
\hline $\mathrm{H}$ & 7.03065500 & 5.56324300 & -3.73655200 \\
\hline $\mathrm{H}$ & 8.67925400 & 5.53876800 & -3.40302300 \\
\hline $\mathrm{H}$ & 6.47634500 & 6.54256000 & -1.69679000 \\
\hline $\mathrm{H}$ & 8.04608000 & 4.06727300 & -1.48659900 \\
\hline $\mathrm{H}$ & 8.37666600 & 5.08280600 & -0.08734000 \\
\hline $\mathrm{H}$ & -2.86335200 & 5.29785000 & -1.79627900 \\
\hline $\mathrm{H}$ & -2.56976600 & 4.55032600 & -3.37378100 \\
\hline $\mathrm{H}$ & 0.24607300 & 6.38265200 & -4.44868200 \\
\hline $\mathrm{H}$ & 0.27480800 & 4.76485300 & -3.91126500 \\
\hline $\mathrm{H}$ & -1.58821600 & 6.91223000 & -3.02551000 \\
\hline $\mathrm{H}$ & -9.59684100 & 5.95169700 & 5.86259800 \\
\hline $\mathrm{H}$ & 0.90254800 & 6.28865900 & -1.70798200 \\
\hline $\mathrm{H}$ & 8.38286700 & 8.16158000 & -1.98628900 \\
\hline $\mathrm{H}$ & 4.29497600 & -3.04965600 & 5.01962000 \\
\hline $\mathrm{O}$ & -1.23435600 & 0.90917700 & -0.15195200 \\
\hline $\mathrm{H}$ & -1.19555400 & -4.92574300 & 2.06655200 \\
\hline $\mathrm{C}$ & -0.15567100 & 1.01520800 & 3.03231200 \\
\hline $\mathrm{C}$ & 0.36627900 & 1.95682100 & 2.44750700 \\
\hline $\mathrm{H}$ & -0.59295200 & 0.18925600 & 3.54955500 \\
\hline $\mathrm{H}$ & 0.81598500 & 2.78137500 & 1.91134200 \\
\hline $\mathrm{H}$ & -0.93183600 & 0.93158400 & 0.77900600 \\
\hline $\mathrm{O}$ & 1.33775700 & 4.24405300 & 0.58681600 \\
\hline $\mathrm{H}$ & -0.69711700 & 0.29560600 & -0.71782800 \\
\hline $\mathrm{H}$ & -1.81094500 & 1.93910200 & -0.78785500 \\
\hline $\mathrm{H}$ & 0.85155900 & 3.89885100 & -0.21441600 \\
\hline $\mathrm{H}$ & 0.92834000 & 5.09348300 & 0.85132400 \\
\hline
\end{tabular}




\begin{tabular}{|c|c|c|c|}
\hline \multicolumn{4}{|c|}{ LE-TS, $E=-3405.333228$} \\
\hline $\mathrm{N}$ & 0.24940900 & 4.93204900 & -4.24892300 \\
\hline $\mathrm{C}$ & -0.54264400 & 5.47350800 & -3.12963700 \\
\hline $\mathrm{C}$ & 0.26914100 & 5.77921700 & -1.83656600 \\
\hline $\mathrm{O}$ & -0.20736900 & 6.27425800 & -0.79365200 \\
\hline $\mathrm{C}$ & -1.71852400 & 4.53376300 & -2.77289600 \\
\hline $\mathrm{C}$ & -1.27413800 & 3.38243700 & -1.86896300 \\
\hline $\mathrm{O}$ & -0.03134700 & 3.35257800 & -1.51738000 \\
\hline $\mathrm{O}$ & -2.17773300 & 2.54609400 & -1.47251800 \\
\hline $\mathrm{N}$ & -3.51394600 & -6.34176000 & 0.27804500 \\
\hline $\mathrm{C}$ & -2.61961500 & -5.17707200 & 0.49924500 \\
\hline $\mathrm{C}$ & -2.90330000 & -4.60379900 & 1.88135900 \\
\hline $\mathrm{O}$ & -3.88393100 & -4.94300800 & 2.57533100 \\
\hline $\mathrm{C}$ & -2.69411200 & -3.97852800 & -0.55566100 \\
\hline $\mathrm{S}$ & -1.39177900 & -2.60711100 & -0.21767900 \\
\hline $\mathrm{N}$ & 8.27411100 & 4.99828000 & -3.21061900 \\
\hline $\mathrm{C}$ & 7.93768900 & 5.29641000 & -1.79590700 \\
\hline $\mathrm{C}$ & 8.86557300 & 6.44585200 & -1.37279400 \\
\hline $\mathrm{O}$ & 9.45222800 & 6.54660900 & -0.28162200 \\
\hline $\mathrm{C}$ & 7.96291400 & 4.11378600 & -0.78503100 \\
\hline $\mathrm{C}$ & 6.63731400 & 3.65548900 & -0.21153600 \\
\hline $\mathrm{C}$ & 5.33248700 & 3.98987300 & -0.55111300 \\
\hline $\mathrm{C}$ & 6.53816600 & 2.68660100 & 0.86623000 \\
\hline $\mathrm{N}$ & 4.42752000 & 3.28196700 & 0.25356600 \\
\hline $\mathrm{C}$ & 5.14855400 & 2.46773300 & 1.12752600 \\
\hline $\mathrm{C}$ & 7.50377000 & 1.98531500 & 1.62842400 \\
\hline $\mathrm{C}$ & 4.70788000 & 1.55615200 & 2.10943100 \\
\hline $\mathrm{C}$ & 7.06879100 & 1.09192200 & 2.61617400 \\
\hline $\mathrm{C}$ & 5.68212700 & 0.87359100 & 2.85292100 \\
\hline $\mathrm{N}$ & 4.04202000 & -0.88331900 & 8.14804900 \\
\hline $\mathrm{C}$ & 3.48992000 & -2.03823300 & 7.45337500 \\
\hline $\mathrm{C}$ & 4.16256000 & -2.19129000 & 6.09866400 \\
\hline $\mathrm{O}$ & 4.88261000 & -1.30538100 & 5.59361100 \\
\hline $\mathrm{C}$ & 1.92083500 & -1.96730700 & 7.24902000 \\
\hline $\mathrm{C}$ & 1.29940500 & -3.20956600 & 6.66304700 \\
\hline $\mathrm{C}$ & 0.75065700 & -4.26650600 & 7.37493100 \\
\hline $\mathrm{C}$ & 1.18527700 & -3.55643000 & 5.25400800 \\
\hline $\mathrm{N}$ & 0.31067600 & -5.25643200 & 6.49118200 \\
\hline $\mathrm{C}$ & 0.56877300 & -4.84506100 & 5.18039100 \\
\hline $\mathrm{C}$ & 1.54189100 & -2.89757200 & 4.04961300 \\
\hline $\mathrm{C}$ & 0.31973600 & -5.49311000 & 3.95512300 \\
\hline $\mathrm{C}$ & 1.29454600 & -3.53277100 & 2.82604500 \\
\hline $\mathrm{C}$ & 0.69439300 & -4.82164900 & 2.78241800 \\
\hline $\mathrm{N}$ & -8.54662500 & 6.36596200 & 2.85032400 \\
\hline $\mathrm{C}$ & -9.09194300 & 5.16885000 & 3.49837300 \\
\hline $\mathrm{C}$ & -8.97624200 & 5.47816000 & 4.97907200 \\
\hline $\mathrm{O}$ & -8.52681300 & 4.70803400 & 5.84826500 \\
\hline $\mathrm{C}$ & -8.47224200 & 3.80443600 & 3.09577000 \\
\hline $\mathrm{C}$ & -8.67747000 & 3.46833600 & 1.63596800 \\
\hline $\mathrm{C}$ & -9.88992700 & 3.31783100 & 0.97714900 \\
\hline
\end{tabular}




$\begin{array}{lccc}\mathrm{C} & -7.63862700 & 3.22147300 & 0.64882400 \\ \mathrm{~N} & -9.66231500 & 2.99371100 & -0.36708900 \\ \mathrm{C} & -8.28545100 & 2.92511200 & -0.59311600 \\ \mathrm{C} & -6.22198100 & 3.20709600 & 0.70329800 \\ \mathrm{C} & -7.56533000 & 2.62033100 & -1.76509500 \\ \mathrm{C} & -5.49724700 & 2.90360500 & -0.45655900 \\ \mathrm{C} & -6.16568800 & 2.61336400 & -1.67932700 \\ \mathrm{C} & -2.89438900 & -3.62975000 & -5.72359300 \\ \mathrm{O} & -2.76368100 & -4.88940300 & -6.43766400 \\ \mathrm{C} & -1.84785700 & -3.50698000 & -4.64536900 \\ \mathrm{~S} & -1.54767200 & -1.87886300 & -3.97322400 \\ \mathrm{C} & -1.10750300 & -4.57712000 & -4.24731900 \\ \mathrm{~S} & 0.15113200 & -4.35864500 & -3.00560300 \\ \mathrm{C} & -1.27958300 & -5.97055600 & -4.82745600 \\ \mathrm{C} & -2.62437900 & -6.04614300 & -5.56367200 \\ \mathrm{C} & 4.58366900 & -1.42477400 & -1.30499300 \\ \mathrm{O} & 5.33878700 & -0.72357500 & -2.33653300 \\ \mathrm{C} & 3.10288900 & -1.23902300 & -1.55241900 \\ \mathrm{~S} & 2.07124300 & -2.65365100 & -1.26830800 \\ \mathrm{C} & 2.62006100 & -0.14210200 & -2.19486100 \\ \mathrm{~S} & 0.89178300 & -0.07294700 & -2.69802500 \\ \mathrm{C} & 3.53346200 & 0.96326900 & -2.70299600 \\ \mathrm{C} & 5.01200200 & 0.70292900 & -2.31931600 \\ \mathrm{~W} & -0.04381100 & -2.17907300 & -2.19537000 \\ \mathrm{H} & -0.45853700 & -6.18786300 & -5.52603600 \\ \mathrm{H} & -1.23386600 & -6.72842500 & -4.03120900 \\ \mathrm{H} & -10.37827300 & 2.81175900 & -1.05496900 \\ \mathrm{H} & -10.89742400 & 3.39579700 & 1.36239000 \\ \mathrm{H} & -5.69839400 & 3.41294200 & 1.63437500 \\ \mathrm{H} & -4.41161000 & 2.87357100 & -0.44347600 \\ \mathrm{H} & -2.68771900 & -6.91945200 & -6.22121900 \\ \mathrm{H} & -3.91074700 & -2.84841700 & -6.48203100 \\ \mathrm{H} & 4.88886900 & -1.05684600 & -5.29636600 \\ \mathrm{H} & 4.86074600 & -2.47927000 & -0.30947900 \\ \mathrm{H} & 5.22343900 & 1.10366300 & -1.31756300 \\ \mathrm{H} & 5.69375400 & 1.16797500 & -3.03912200 \\ \mathrm{H} & 3.22405600 & 1.94223100 & -2.30706400 \\ \mathrm{H} & 3.42563300 & 1.01432300 & -3.79691200 \\ \mathrm{H} & -3.68588200 & -3.51019600 & -0.53555300 \\ \mathrm{H} & -2.49188600 & -4.36676700 & -1.55749300 \\ \mathrm{H} & -4.38794500 & -6.28529800 & 0.79475400 \\ \mathrm{H} & -3.59580400 & -6.65550300 & -0.68329400 \\ \mathrm{H} & -1.57057800 & -5.50793200 & 0.48978100 \\ \mathrm{H} & -8.83868200 & 6.48838200 & 1.88378700 \\ \mathrm{H} & -7.53643900 & 6.46021700 & 2.94209700 \\ \mathrm{H} & -7.39960700 & 3.82756500 & 3.32716900 \\ \mathrm{H} & -8.89869400 & 3.02931000 & 3.74747000 \\ \mathrm{H} & & & \\ \mathrm{H} & & & \end{array}$




$\begin{array}{lrrr}\mathrm{H} & -5.56954400 & 2.37023900 & -2.55525000 \\ \mathrm{H} & -8.06939400 & 2.38874400 & -2.70106000 \\ \mathrm{H} & 3.46014700 & -0.45512800 & 8.85723400 \\ \mathrm{H} & 4.56235700 & -0.23982200 & 7.55935400 \\ \mathrm{H} & 3.68344400 & -2.97312800 & 8.00710600 \\ \mathrm{H} & 1.70074000 & -1.08589400 & 6.63113000 \\ \mathrm{H} & 1.49584600 & -1.77299000 & 8.24410800 \\ \mathrm{H} & 1.99917300 & -1.91057400 & 4.06484300 \\ \mathrm{H} & 1.55258400 & -3.04491200 & 1.88981300 \\ \mathrm{H} & 0.52892700 & -5.28766800 & 1.81488300 \\ \mathrm{H} & -0.14964200 & -6.47336500 & 3.91276100 \\ \mathrm{H} & -0.15545000 & -6.11274700 & 6.75316600 \\ \mathrm{H} & 0.63626200 & -4.38232700 & 8.44376300 \\ \mathrm{H} & 5.38092100 & 0.15967300 & 3.61584600 \\ \mathrm{H} & 7.79583700 & 0.54347500 & 3.21086500 \\ \mathrm{H} & 8.56684000 & 2.13378400 & 1.45044200 \\ \mathrm{H} & 3.65069800 & 1.36256400 & 2.26753900 \\ \mathrm{H} & 3.41073100 & 3.34003900 & 0.21399000 \\ \mathrm{H} & 4.96610900 & 4.67206000 & -1.30569800 \\ \mathrm{H} & 7.51294900 & 4.58724200 & -3.74434600 \\ \mathrm{H} & 9.13935600 & 4.47435200 & -3.32561600 \\ \mathrm{H} & 6.93562400 & 5.75362900 & -1.80913600 \\ \mathrm{H} & 8.45520600 & 3.25905100 & -1.27524500 \\ \mathrm{H} & 8.61620000 & 4.40141200 & 0.05161600 \\ \mathrm{H} & -2.50150100 & 5.07817400 & -2.23191100 \\ \mathrm{H} & -2.16509300 & 4.13419200 & -3.68920900 \\ \mathrm{H} & 0.92076600 & 5.56764400 & -4.66832700 \\ \mathrm{H} & 0.61481600 & 3.99574900 & -4.08890100 \\ \mathrm{H} & -0.97494000 & 6.43152200 & -3.45792300 \\ \mathrm{H} & -9.31146700 & 6.50235800 & 5.23452400 \\ \mathrm{H} & 1.35748600 & 5.58662800 & -1.94087700 \\ \mathrm{H} & 8.98414300 & 7.21035300 & -2.16585600 \\ \mathrm{H} & 3.97315100 & -3.13656600 & 5.56743600 \\ \mathrm{H} & -1.61929100 & 0.83601800 & 0.15258500 \\ \mathrm{H} & -2.19733300 & -3.83216800 & 2.22630400 \\ \mathrm{C} & 0.07481600 & 1.50123500 & 2.05660600 \\ \mathrm{H} & 0.98997600 & 2.38868200 & 2.14603100 \\ \mathrm{H} & -0.20117000 & 0.79394800 & 2.83823400 \\ \mathrm{H} & 1.79161100 & 2.91603900 & 2.63109300 \\ \mathrm{H} & -1.67502000 & -0.12243800 & -0.06638500 \\ \mathrm{H} & 1.28040800 & 3.48328700 & 0.58291600 \\ \mathrm{H} & -1.871717000 & 1.13016500 & 0.89969300 \\ \mathrm{H} & 1.01667100 & 4.40613900 & 0.79655400\end{array}$

LE-EP1, $E=-3405.437405$

$\begin{array}{lrrr}\mathrm{N} & 0.43888400 & 5.36851300 & -4.26316100 \\ \mathrm{C} & -0.32967600 & 5.80642600 & -3.06926900 \\ \mathrm{C} & 0.55521900 & 6.12210300 & -1.82264500\end{array}$




\begin{tabular}{|c|c|c|c|}
\hline $\mathrm{O}$ & 0.54339600 & 5.46567500 & -0.76577300 \\
\hline $\mathrm{C}$ & -1.45595800 & 4.80383800 & -2.73488100 \\
\hline $\mathrm{C}$ & -1.05480000 & 3.41292700 & -2.25555400 \\
\hline $\mathrm{O}$ & 0.00439300 & 2.87172200 & -2.93552000 \\
\hline $\mathrm{O}$ & -1.67844000 & 2.80323100 & -1.37309200 \\
\hline $\mathrm{N}$ & -3.96132700 & -5.80431400 & -0.07256100 \\
\hline $\mathrm{C}$ & -2.86312000 & -4.94115000 & 0.34158700 \\
\hline $\mathrm{C}$ & -2.83856300 & -4.84317300 & 1.85933000 \\
\hline $\mathrm{O}$ & -3.71753400 & -5.34112500 & 2.59287500 \\
\hline $\mathrm{C}$ & -2.93694600 & -3.48911000 & -0.25648400 \\
\hline S & -1.34577000 & -2.51604000 & 0.01074200 \\
\hline $\mathrm{N}$ & 8.46819400 & 4.88568500 & -3.38262100 \\
\hline $\mathrm{C}$ & 8.20277800 & 5.12355900 & -1.94246700 \\
\hline $\mathrm{C}$ & 9.18351800 & 6.22314000 & -1.50580900 \\
\hline $\mathrm{O}$ & 9.88656200 & 6.20897100 & -0.48119200 \\
\hline $\mathrm{C}$ & 8.22171300 & 3.87891700 & -1.00976000 \\
\hline $\mathrm{C}$ & 6.96425100 & 3.62049100 & -0.20525300 \\
\hline $\mathrm{C}$ & 5.73672300 & 4.27325000 & -0.22113600 \\
\hline $\mathrm{C}$ & 6.83675900 & 2.55061600 & 0.76731800 \\
\hline $\mathrm{N}$ & 4.85400500 & 3.67144600 & 0.68109900 \\
\hline $\mathrm{C}$ & 5.50806700 & 2.60882800 & 1.29931800 \\
\hline $\mathrm{C}$ & 7.72361300 & 1.54911900 & 1.23216400 \\
\hline $\mathrm{C}$ & 5.05323000 & 1.69291400 & 2.27029700 \\
\hline $\mathrm{C}$ & 7.27367600 & 0.64148900 & 2.20079600 \\
\hline $\mathrm{C}$ & 5.94808300 & 0.70949900 & 2.71656500 \\
\hline $\mathrm{N}$ & 4.06631500 & -1.13473400 & 7.83794700 \\
\hline $\mathrm{C}$ & 3.53234900 & -2.30980100 & 7.16232500 \\
\hline $\mathrm{C}$ & 4.06668200 & -2.37033500 & 5.74066700 \\
\hline $\mathrm{O}$ & 4.68101300 & -1.42555800 & 5.20486600 \\
\hline $\mathrm{C}$ & 1.94982600 & -2.37142700 & 7.12193600 \\
\hline $\mathrm{C}$ & 1.38858500 & -3.67506400 & 6.61104600 \\
\hline $\mathrm{C}$ & 1.00248600 & -4.75602900 & 7.39013700 \\
\hline $\mathrm{C}$ & 1.19022000 & -4.07396200 & 5.22472900 \\
\hline $\mathrm{N}$ & 0.58518300 & -5.80893900 & 6.57028900 \\
\hline $\mathrm{C}$ & 0.69526500 & -5.41646700 & 5.23339300 \\
\hline $\mathrm{C}$ & 1.38107100 & -3.42393700 & 3.97905200 \\
\hline $\mathrm{C}$ & 0.41163300 & -6.12426500 & 4.04971800 \\
\hline $\mathrm{C}$ & 1.10323500 & -4.12107000 & 2.79642100 \\
\hline $\mathrm{C}$ & 0.62784500 & -5.46132500 & 2.83298300 \\
\hline $\mathrm{N}$ & -8.12126300 & 7.09277900 & 3.05643100 \\
\hline $\mathrm{C}$ & -8.67344600 & 5.87883800 & 3.67356400 \\
\hline $\mathrm{C}$ & -8.56972600 & 6.15526700 & 5.15982900 \\
\hline $\mathrm{O}$ & -8.17537800 & 5.35035400 & 6.02363100 \\
\hline $\mathrm{C}$ & -8.05122300 & 4.52851000 & 3.25288100 \\
\hline $\mathrm{C}$ & -8.29568600 & 4.16574500 & 1.80658700 \\
\hline $\mathrm{C}$ & -9.37893600 & 4.50127300 & 1.00654300 \\
\hline $\mathrm{C}$ & -7.42529300 & 3.33059400 & 0.99595700 \\
\hline $\mathrm{N}$ & -9.22846400 & 3.91840200 & -0.26127200 \\
\hline $\mathrm{C}$ & -8.03286900 & 3.19336000 & -0.29084200 \\
\hline $\mathrm{C}$ & -6.18672000 & 2.68904100 & 1.24587200 \\
\hline
\end{tabular}




\begin{tabular}{|c|c|c|c|}
\hline & -7.44022300 & 2.43984400 & -1.32330300 \\
\hline 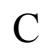 & -5.58388400 & 1.93857200 & 0.22739400 \\
\hline $\mathrm{C}$ & -6.21243600 & 1.81901600 & -1.04516100 \\
\hline $\mathrm{C}$ & -3.24877600 & -3.00135300 & -5.83866500 \\
\hline U & -3.23691300 & -4.15300100 & -6.74499500 \\
\hline $\mathrm{C}$ & -2.33121700 & -3.19888400 & -4.64592100 \\
\hline S & -1.83412600 & -1.73178200 & -3.73752100 \\
\hline C & -1.82772800 & -4.41922800 & -4.32243200 \\
\hline S & -0.59682100 & -4.54962600 & -3.02539300 \\
\hline $\mathrm{C}$ & -2.16408200 & -5.67238500 & -5.11582600 \\
\hline $\mathrm{C}$ & -3.38061600 & -5.41824500 & -6.02562300 \\
\hline $\mathrm{C}$ & 4.50296800 & -1.81755200 & -2.06382000 \\
\hline $\mathrm{O}$ & 5.18701200 & -0.66662100 & -2.65769400 \\
\hline $\mathrm{C}$ & 2.99921800 & -1.60554400 & -1.99673900 \\
\hline $\mathrm{S}$ & 1.97421000 & -3.07052600 & -1.89748300 \\
\hline $\mathrm{C}$ & 2.44888200 & -0.36939600 & -2.11565200 \\
\hline $\mathrm{S}$ & 0.66380500 & -0.23636400 & -2.25185200 \\
\hline $\mathrm{C}$ & 3.29202800 & 0.88608200 & -2.27391100 \\
\hline $\mathrm{C}$ & 4.77935000 & 0.57789600 & -1.99826000 \\
\hline W & -0.28960200 & -2.41064300 & -2.08912300 \\
\hline $\mathrm{H}$ & -1.29509300 & -5.96200600 & -5.72433100 \\
\hline $\mathrm{H}$ & -2.37456200 & -6.51406500 & -4.43892200 \\
\hline $\mathrm{H}$ & -4.30830800 & -5.39965300 & -5.42645900 \\
\hline $\mathrm{H}$ & -3.47254300 & -6.18509600 & -6.80183900 \\
\hline $\mathrm{H}$ & -2.91640700 & -2.15228300 & -6.44548000 \\
\hline $\mathrm{H}$ & -4.28562500 & -2.80584900 & -5.50969300 \\
\hline $\mathrm{H}$ & 4.91968300 & -2.01325900 & -1.06021500 \\
\hline $\mathrm{H}$ & 4.74051800 & -2.66823700 & -2.71109300 \\
\hline $\mathrm{H}$ & 4.95866700 & 0.49621400 & -0.91505700 \\
\hline $\mathrm{H}$ & 5.43213000 & 1.35669200 & -2.40406400 \\
\hline $\mathrm{H}$ & 2.95490500 & 1.67016600 & -1.57958900 \\
\hline $\mathrm{H}$ & 3.17075000 & 1.27903900 & -3.29502600 \\
\hline $\mathrm{H}$ & -3.75309000 & -2.91416400 & 0.19932900 \\
\hline $\mathrm{H}$ & -3.10812700 & -3.56022800 & -1.33286900 \\
\hline $\mathrm{H}$ & -4.70675500 & -5.92455400 & 0.60382900 \\
\hline $\mathrm{H}$ & -4.22118600 & -5.78750600 & -1.04955500 \\
\hline $\mathrm{H}$ & -1.89347400 & -5.35271500 & 0.01709100 \\
\hline $\mathrm{H}$ & -8.37625200 & 7.20942200 & 2.07814400 \\
\hline $\mathrm{H}$ & -7.11314200 & 7.18323200 & 3.17777800 \\
\hline $\mathrm{H}$ & -9.75338400 & 5.86997300 & 3.44922000 \\
\hline $\mathrm{H}$ & -6.96962800 & 4.56814900 & 3.44552100 \\
\hline $\mathrm{H}$ & -8.43730900 & 3.74516400 & 3.92149800 \\
\hline $\mathrm{H}$ & -9.87980800 & 4.00681600 & -1.02697700 \\
\hline $\mathrm{H}$ & -10.25442000 & 5.09376500 & 1.23449700 \\
\hline $\mathrm{H}$ & -5.70177700 & 2.77072000 & 2.21616400 \\
\hline $\mathrm{H}$ & -4.62671400 & 1.44673800 & 0.39866200 \\
\hline $\mathrm{H}$ & -5.72109000 & 1.23026200 & -1.81666900 \\
\hline $\mathrm{H}$ & -7.91070200 & 2.33882900 & -2.29947600 \\
\hline П & 3.52547800 & -0.76627500 & 8.61038100 \\
\hline П & 319200 & -0.44125500 & 7.22314700 \\
\hline
\end{tabular}




\begin{tabular}{lrrr} 
& & & \\
$\mathrm{H}$ & 3.85787100 & -3.24117900 & 7.65755900 \\
$\mathrm{H}$ & 1.58761500 & -1.52124600 & 6.52796400 \\
$\mathrm{H}$ & 1.61357100 & -2.20457000 & 8.15512200 \\
$\mathrm{H}$ & 1.72930200 & -2.39486000 & 3.92944000 \\
$\mathrm{H}$ & 1.23796200 & -3.63533500 & 1.83416800 \\
$\mathrm{H}$ & 0.43046800 & -5.97657800 & 1.89613600 \\
$\mathrm{H}$ & 0.03219200 & -7.14327100 & 4.07102000 \\
$\mathrm{H}$ & 0.22582100 & -6.69608600 & 6.89099700 \\
$\mathrm{H}$ & 0.99047900 & -4.84947000 & 8.46719000 \\
$\mathrm{H}$ & 5.62356000 & -0.01349000 & 3.46063500 \\
$\mathrm{H}$ & 7.94128600 & -0.13567000 & 2.56684600 \\
$\mathrm{H}$ & 8.73801400 & 1.48125800 & 0.84385900 \\
$\mathrm{H}$ & 4.03779700 & 1.73316300 & 2.65557000 \\
$\mathrm{H}$ & 3.89092700 & 3.96819100 & 0.87106700 \\
$\mathrm{H}$ & 5.41383400 & 5.12861300 & -0.79813900 \\
$\mathrm{H}$ & 7.67579500 & 4.50585900 & -3.89353600 \\
$\mathrm{H}$ & 9.32041000 & 4.35898100 & -3.56341400 \\
$\mathrm{H}$ & 7.21621300 & 5.60866900 & -1.90097400 \\
$\mathrm{H}$ & 8.43616100 & 2.99478300 & -1.62963500 \\
$\mathrm{H}$ & 9.06828000 & 3.98153700 & -0.31463100 \\
$\mathrm{H}$ & -2.11648600 & 5.21313700 & -1.96492500 \\
$\mathrm{H}$ & -2.04547500 & 4.66687000 & -3.65079800 \\
$\mathrm{H}$ & 1.06998100 & 6.04701900 & -4.67677000 \\
$\mathrm{H}$ & 0.78506900 & 4.41321700 & -4.19576500 \\
$\mathrm{H}$ & -0.81663100 & 6.75896100 & -3.33279000 \\
$\mathrm{H}$ & -8.86468000 & 7.18877000 & 5.42781600 \\
$\mathrm{H}$ & 1.27627300 & 6.95374900 & -1.96051100 \\
$\mathrm{H}$ & 9.23091500 & 7.06342100 & -2.22688300 \\
$\mathrm{O}$ & 3.88247200 & -3.30672000 & 5.19293100 \\
$\mathrm{H}$ & -2.58969500 & 0.80759100 & 0.42751800 \\
$\mathrm{C}$ & -1.99947300 & -4.26845500 & 2.28343400 \\
$\mathrm{C}$ & 0.14063700 & 2.94663200 & 1.81674600 \\
$\mathrm{H}$ & 1.39079700 & 3.39462500 & 2.03520600 \\
$\mathrm{H}$ & -0.29243000 & 2.19559000 & 2.46794300 \\
$\mathrm{H}$ & 2.01602900 & 3.05672400 & 2.85610300 \\
$\mathrm{O}$ & -2.16814600 & -0.06739300 & 0.29413200 \\
$\mathrm{H}$ & 2.07503700 & 4.34627400 & 1.25907400 \\
$\mathrm{H}$ & -0.46885500 & 3.27823300 & 0.98070600 \\
& -2.21940600 & 1.46479300 & -0.20181500 \\
& 0.19325500 & 1.90299100 & -2.70338900 \\
\hline & 1.49614100 & 4.77050300 & 0.5783060
\end{tabular}

\begin{tabular}{lrrr}
\multicolumn{4}{l}{$\mathbf{L N}-\mathbf{E}, E=-3251.053917$} \\
$\mathrm{~N}$ & 2.98658500 & 3.92058500 & -4.51201500 \\
$\mathrm{C}$ & 1.91877500 & 4.40375200 & -3.58104000 \\
$\mathrm{C}$ & 2.46328400 & 5.14628900 & -2.33010000 \\
$\mathrm{O}$ & 2.31977200 & 6.37808200 & -2.15987300 \\
$\mathrm{C}$ & 1.03130600 & 3.21278600 & -3.14456500 \\
$\mathrm{C}$ & 1.67354400 & 2.32612500 & -2.05266200 \\
$\mathrm{O}$ & 2.96541700 & 2.23577300 & -2.06521600
\end{tabular}




\begin{tabular}{|c|c|c|c|}
\hline $\mathrm{O}$ & 0.89045500 & 1.77580500 & -1.18917900 \\
\hline $\mathrm{N}$ & -5.78364200 & -4.37396100 & -0.27169400 \\
\hline $\mathrm{C}$ & -4.62722400 & -3.68698400 & 0.30945500 \\
\hline $\mathrm{C}$ & -4.96878700 & -3.19199200 & 1.69830400 \\
\hline $\mathrm{O}$ & -6.11961300 & -3.24430800 & 2.19268300 \\
\hline $\mathrm{C}$ & -4.09381500 & -2.46706900 & -0.5307630 \\
\hline $\mathrm{S}$ & -2.42601400 & -1.88135100 & 0.07298200 \\
\hline $\mathrm{N}$ & 9.86397000 & 1.36303100 & -1.67400900 \\
\hline $\mathrm{C}$ & 9.36702400 & 2.10137900 & -0.4805580 \\
\hline $\mathrm{C}$ & 10.57718700 & 2.81437300 & 0.116986 \\
\hline $\mathrm{O}$ & 10.80615900 & 2.99963400 & 1.326545 \\
\hline $\mathrm{C}$ & 8.56176200 & 1.31178800 & 0.58529200 \\
\hline $\mathrm{C}$ & 7.05758500 & 1.39806200 & 0.4664820 \\
\hline $\mathrm{C}$ & 6.27766400 & 1.84531500 & -0.5985370 \\
\hline $\mathrm{C}$ & 6.12626400 & 0.98592500 & $1.4981940 \mathrm{C}$ \\
\hline $\mathrm{N}$ & 4.92563600 & 1.74266400 & -0.29144400 \\
\hline $\mathrm{C}$ & 4.80494400 & 1.20857600 & 0.9850780 \\
\hline $\mathrm{C}$ & 6.27849000 & 0.44048800 & 2.79804500 \\
\hline $\mathrm{C}$ & 3.64644500 & 0.88006100 & 1.72032200 \\
\hline $\mathrm{C}$ & 5.13055100 & 0.12259200 & 3.53695300 \\
\hline $\mathrm{C}$ & 3.82777900 & 0.33557800 & 2.99928200 \\
\hline $\mathrm{N}$ & 1.52754800 & -0.58553100 & 8.72937800 \\
\hline $\mathrm{C}$ & 0.76683000 & -1.65329800 & 8.08761000 \\
\hline $\mathrm{C}$ & 1.55281500 & -2.19282600 & 6.90148700 \\
\hline $\mathrm{O}$ & 2.61629900 & -1.67366700 & 6.50551700 \\
\hline $\mathrm{C}$ & -0.67849400 & -1.22177900 & 7.62155100 \\
\hline $\mathrm{C}$ & -1.53267700 & -2.36262600 & 7.12836600 \\
\hline $\mathrm{C}$ & -2.41029500 & -3.11968200 & 7.89103100 \\
\hline $\mathrm{C}$ & -1.56213900 & -2.92037100 & 5.7839680 \\
\hline $\mathrm{N}$ & -2.97704400 & -4.12589300 & 7.1007010 \\
\hline $\mathrm{C}$ & -2.46916800 & -4.02471200 & 5.8001290 \\
\hline $\mathrm{C}$ & -0.91376400 & -2.58178000 & 4.5683590 \\
\hline $\mathrm{C}$ & -2.72885700 & -4.80325900 & 4.6553300 \\
\hline $\mathrm{C}$ & -1.16949600 & -3.34579100 & 3.42364200 \\
\hline $\mathrm{C}$ & -2.06558200 & -4.45003600 & 3.47151000 \\
\hline $\mathrm{N}$ & -6.03026400 & 9.53159800 & -0.1933580 \\
\hline $\mathrm{C}$ & -6.42912000 & 8.47544200 & 0.75528000 \\
\hline $\mathrm{C}$ & -7.18288500 & 9.23761800 & 1.82876400 \\
\hline $\mathrm{O}$ & -7.04251600 & 9.10761800 & 3.05920300 \\
\hline $\mathrm{C}$ & -5.31672100 & 7.55557500 & 1.3051300 \\
\hline $\mathrm{C}$ & -4.82173100 & 6.50979700 & 0.33072300 \\
\hline $\mathrm{C}$ & -5.46949600 & 5.98927100 & -0.7821280 \\
\hline $\mathrm{C}$ & -3.57218000 & 5.77711200 & 0.4591140 \\
\hline $\mathrm{N}$ & -4.68980400 & 4.97379200 & $-1.350942 C$ \\
\hline $\mathrm{C}$ & -3.51978500 & 4.82079800 & -0.6028870 \\
\hline $\mathrm{C}$ & -2.49634900 & 5.83563500 & 1.3797080 \\
\hline $\mathrm{C}$ & -2.44201100 & 3.92847600 & -0.7675210 \\
\hline $\mathrm{C}$ & -1.41785100 & 4.95582200 & 1.2163340 \\
\hline $\mathrm{C}$ & -1.38791800 & 4.00810400 & 0.1539810 \\
\hline $\mathrm{C}$ & -3.13820100 & -3.12477600 & -6.1133980 \\
\hline
\end{tabular}




\begin{tabular}{|c|c|c|c|}
\hline $\mathrm{O}$ & -3.20477600 & -4.47864500 & -6.68347300 \\
\hline $\mathrm{C}$ & -2.57163800 & -3.11098600 & -4.70637800 \\
\hline $\mathrm{S}$ & -1.86041700 & -1.58017700 & -4.12690600 \\
\hline$C$ & -2.45782000 & -4.24816900 & -3.96396000 \\
\hline $\mathrm{S}$ & -1.62531100 & -4.21710500 & -2.38039100 \\
\hline $\mathrm{C}$ & -2.92351100 & -5.59835700 & -4.49065500 \\
\hline $\mathrm{C}$ & -3.80432300 & -5.42389900 & -5.74160900 \\
\hline $\mathrm{C}$ & 3.61257100 & -2.87094000 & -0.31241900 \\
\hline 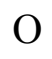 & 4.86798000 & -2.73393400 & -1.06139200 \\
\hline $\mathrm{C}$ & 2.40588800 & -2.41866200 & -1.11494600 \\
\hline $\mathrm{S}$ & 0.79145100 & -2.99134100 & -0.60617300 \\
\hline $\mathrm{C}$ & 2.53347300 & -1.65514500 & -2.23528200 \\
\hline $\mathrm{S}$ & 1.10159100 & -1.14695100 & -3.15468800 \\
\hline $\mathrm{C}$ & 3.89177800 & -1.22500600 & -2.77038700 \\
\hline $\mathrm{C}$ & 4.98223400 & -1.42533900 & -1.70350100 \\
\hline $\mathrm{W}$ & -0.83473200 & -1.99914500 & -2.01157000 \\
\hline $\mathrm{H}$ & -2.04381900 & -6.21258100 & -4.73698200 \\
\hline $\mathrm{H}$ & -3.48438500 & -6.14316200 & -3.71520800 \\
\hline $\mathrm{H}$ & -4.80881400 & -5.06433800 & -5.45083800 \\
\hline $\mathrm{H}$ & -3.90929700 & -6.36290400 & -6.29829200 \\
\hline $\mathrm{H}$ & -2.49167600 & -2.55781500 & -6.79180700 \\
\hline $\mathrm{H}$ & -4.15030900 & -2.67805200 & -6.14338600 \\
\hline $\mathrm{H}$ & 3.69224800 & -2.29321700 & 0.62521900 \\
\hline $\mathrm{H}$ & 3.54065700 & -3.93500100 & -0.05950900 \\
\hline $\mathrm{H}$ & 4.91116900 & -0.64148900 & -0.94121200 \\
\hline $\mathrm{H}$ & 5.98707500 & -1.40709500 & -2.14264600 \\
\hline $\mathrm{H}$ & 3.85537300 & -0.16510000 & -3.05799000 \\
\hline $\mathrm{H}$ & 4.12948900 & -1.80870200 & -3.67364800 \\
\hline $\mathrm{H}$ & -4.81787300 & -1.64010900 & -0.49900600 \\
\hline $\mathrm{H}$ & -3.98246200 & -2.80111100 & -1.56635800 \\
\hline $\mathrm{H}$ & -6.67596000 & -4.09275600 & 0.12526100 \\
\hline $\mathrm{H}$ & -5.76509600 & -4.45599000 & -1.28231400 \\
\hline $\mathrm{H}$ & -3.76967300 & -4.37200200 & 0.39402000 \\
\hline $\mathrm{H}$ & -5.82227300 & 9.18559000 & -1.12789800 \\
\hline $\mathrm{H}$ & -5.26359700 & 10.10762500 & 0.15273500 \\
\hline $\mathrm{H}$ & -7.19159600 & 7.86313200 & 0.24636200 \\
\hline $\mathrm{H}$ & -4.47642400 & 8.18481100 & 1.63623600 \\
\hline $\mathrm{H}$ & -5.69339200 & 7.07009600 & 2.21792400 \\
\hline $\mathrm{H}$ & -4.93967500 & 4.41981300 & -2.15725300 \\
\hline $\mathrm{H}$ & -6.42924600 & 6.24304300 & -1.21089500 \\
\hline $\mathrm{H}$ & -2.50569000 & 6.54757700 & 2.20293900 \\
\hline $\mathrm{H}$ & -0.58166000 & 4.98879400 & 1.91078900 \\
\hline $\mathrm{H}$ & -0.54205700 & 3.33277300 & 0.04475000 \\
\hline $\mathrm{H}$ & -2.41798200 & 3.19307000 & -1.56816400 \\
\hline $\mathrm{H}$ & 0.98254700 & 0.15369900 & 9.15786800 \\
\hline $\mathrm{H}$ & 2.32650600 & -0.26697900 & 8.18616600 \\
\hline $\mathrm{H}$ & 0.61953900 & -2.50894500 & 8.77088200 \\
\hline $\mathrm{H}$ & -0.57664400 & -0.44404800 & 6.85256400 \\
\hline $\mathrm{H}$ & -1.15433500 & -0.74993500 & 8.49378500 \\
\hline 1 & -0.23770400 & -1.73182900 & 4.50873500 \\
\hline
\end{tabular}




$\begin{array}{cccc}\mathrm{H} & -0.70742000 & -3.08813700 & 2.47532600 \\ \mathrm{H} & -2.24024600 & -5.01766900 & 2.56146000 \\ \mathrm{H} & -3.42801200 & -5.63646100 & 4.67705500 \\ \mathrm{H} & -3.67925900 & -4.78516500 & 7.40174500 \\ \mathrm{H} & -2.67513500 & -3.01294100 & 8.93402100 \\ \mathrm{H} & 2.96123400 & 0.05090400 & 3.59195100 \\ \mathrm{H} & 5.22213700 & -0.31087500 & 4.53090900 \\ \mathrm{H} & 7.26840500 & 0.25849300 & 3.21420900 \\ \mathrm{H} & 2.65740800 & 1.02204900 & 1.29153000 \\ \mathrm{H} & 4.12861600 & 1.91382300 & -0.94818700 \\ \mathrm{H} & 6.58251800 & 2.22060800 & -1.56661500 \\ \mathrm{H} & 9.14592300 & 1.21424900 & -2.38016600 \\ \mathrm{H} & 10.31184900 & 0.48020700 & -1.42924100 \\ \mathrm{H} & 8.73507600 & 2.92069200 & -0.86246600 \\ \mathrm{H} & 8.88787700 & 0.25796700 & 0.55168500 \\ \mathrm{H} & 8.86237400 & 1.68570100 & 1.57464600 \\ \mathrm{H} & 0.06708500 & 3.57176600 & -2.76921900 \\ \mathrm{H} & 0.83862100 & 2.58421100 & -4.02474300 \\ \mathrm{H} & 3.62170300 & 4.65791300 & -4.81806700 \\ \mathrm{H} & 3.47645700 & 3.12708500 & -4.09086000 \\ \mathrm{H} & 1.30138700 & 5.12996600 & -4.12694400 \\ \mathrm{H} & -7.88681500 & 9.98259900 & 1.40741700 \\ \mathrm{H} & 3.03767900 & 4.53322500 & -1.61706300 \\ \mathrm{H} & 11.28201700 & 3.17550400 & -0.66015900 \\ \mathrm{H} & 1.13887000 & -3.08400000 & 6.40647700 \\ \mathrm{O} & -1.06423100 & 0.11465700 & -1.43664500 \\ \mathrm{H} & -0.28224900 & 0.77244500 & -1.40992400 \\ \mathrm{H} & -1.67759900 & 0.16452200 & -0.66445900 \\ \mathrm{H} & -4.13272600 & -2.72967200 & 2.24553000\end{array}$

LN-ES, $E=-3328.3696645$

$\begin{array}{llcc}\mathrm{N} & 3.05917800 & 4.19386700 & -4.28129100 \\ \mathrm{C} & 1.94928200 & 4.62616200 & -3.37594300 \\ \mathrm{C} & 2.43087000 & 5.32376800 & -2.07536700 \\ \mathrm{O} & 2.25397800 & 6.54419900 & -1.85919900 \\ \mathrm{C} & 1.06170000 & 3.40917200 & -3.02389100 \\ \mathrm{C} & 1.67257400 & 2.47072300 & -1.95700900 \\ \mathrm{O} & 2.96523300 & 2.43124500 & -1.88434400 \\ \mathrm{O} & 0.85727100 & 1.82538400 & -1.19707900 \\ \mathrm{~N} & -5.75891700 & -4.37284600 & -0.73872500 \\ \mathrm{C} & -4.63969000 & -3.66935800 & -0.10985600 \\ \mathrm{C} & -5.13605200 & -2.85360900 & 1.06644500 \\ \mathrm{O} & -6.34827100 & -2.69660300 & 1.33942000 \\ \mathrm{C} & -3.84624200 & -2.72807200 & -1.08466500 \\ \mathrm{~S} & -2.23575900 & -2.17891400 & -0.33016200 \\ \mathrm{~N} & 9.85097600 & 1.60853000 & -1.26781800 \\ \mathrm{C} & 9.29734900 & 2.28917600 & -0.06571500 \\ \mathrm{C} & 10.47083100 & 2.99491700 & 0.60744300 \\ \mathrm{O} & 10.65190500 & 3.12991500 & 1.83169200 \\ \mathrm{C} & 8.46711900 & 1.44319000 & 0.93443300 \\ & & & \mathrm{~S} 27\end{array}$




\begin{tabular}{|c|c|c|c|}
\hline $\mathrm{C}$ & 6.96757700 & 1.52302900 & 0.77092300 \\
\hline $\mathrm{C}$ & 6.21520400 & 2.00699200 & -0.29789500 \\
\hline $\mathrm{C}$ & 6.01152600 & 1.06006600 & 1.75643000 \\
\hline $\mathrm{N}$ & 4.85547600 & 1.87780800 & -0.03534400 \\
\hline $\mathrm{C}$ & 4.70337400 & 1.29202400 & 1.21534400 \\
\hline $\mathrm{C}$ & 6.13442500 & 0.46413200 & 3.03720600 \\
\hline $\mathrm{C}$ & 3.52932500 & 0.92980100 & 1.90828400 \\
\hline $\mathrm{C}$ & 4.97000200 & 0.10829200 & 3.73150600 \\
\hline $\mathrm{C}$ & 3.68028500 & 0.33493600 & 3.16857800 \\
\hline $\mathrm{N}$ & 1.12813700 & -0.86187300 & 8.69874000 \\
\hline $\mathrm{C}$ & 0.45165300 & -1.97004700 & 8.03600600 \\
\hline $\mathrm{C}$ & 1.24986500 & -2.39346600 & 6.81069800 \\
\hline $\mathrm{O}$ & 2.29817500 & -1.81854000 & 6.45676300 \\
\hline $\mathrm{C}$ & -1.04047200 & -1.65448200 & 7.64075100 \\
\hline $\mathrm{C}$ & -1.83569400 & -2.85978200 & 7.20389300 \\
\hline $\mathrm{C}$ & -2.50404100 & -3.74336300 & 8.03893400 \\
\hline $\mathrm{C}$ & -2.02824300 & -3.34454400 & 5.84466500 \\
\hline $\mathrm{N}$ & -3.09825800 & -4.75681100 & 7.27747500 \\
\hline $\mathrm{C}$ & -2.82078400 & -4.53248500 & 5.92405400 \\
\hline $\mathrm{C}$ & -1.61567400 & -2.87659800 & 4.57150900 \\
\hline $\mathrm{C}$ & -3.20100000 & -5.26276600 & 4.78153000 \\
\hline $\mathrm{C}$ & -1.99234400 & -3.59467500 & 3.42908500 \\
\hline $\mathrm{C}$ & -2.77527000 & -4.77777700 & 3.53612500 \\
\hline $\mathrm{N}$ & -6.20262300 & 9.51403700 & -0.11558900 \\
\hline $\mathrm{C}$ & -6.63045000 & 8.41466500 & 0.76918100 \\
\hline $\mathrm{C}$ & -7.43203900 & 9.12440000 & 1.84427200 \\
\hline $\mathrm{O}$ & -7.33673500 & 8.94344100 & 3.07256300 \\
\hline $\mathrm{C}$ & -5.53295400 & 7.47776900 & 1.32020400 \\
\hline $\mathrm{C}$ & -4.98560500 & 6.48863400 & 0.31496900 \\
\hline $\mathrm{C}$ & -5.56776200 & 6.04482800 & -0.86521800 \\
\hline $\mathrm{C}$ & -3.74866500 & 5.73939100 & 0.47026100 \\
\hline $\mathrm{N}$ & -4.75960700 & 5.06144200 & -1.45057300 \\
\hline $\mathrm{C}$ & -3.63706000 & 4.85193400 & -0.64554300 \\
\hline $\mathrm{C}$ & -2.72888700 & 5.73029000 & 1.45439400 \\
\hline $\mathrm{C}$ & -2.55570500 & 3.96246000 & -0.80205400 \\
\hline $\mathrm{C}$ & -1.64751400 & 4.85213200 & 1.30020800 \\
\hline $\mathrm{C}$ & -1.55889400 & 3.97222900 & 0.18405500 \\
\hline $\mathrm{C}$ & -2.91450200 & -2.89250400 & -6.36465500 \\
\hline $\mathrm{O}$ & -2.92135800 & -4.18632100 & -7.03844400 \\
\hline $\mathrm{C}$ & -2.24114400 & -2.98304100 & -5.01774300 \\
\hline S & -1.50594100 & -1.49193200 & -4.39655700 \\
\hline $\mathrm{C}$ & -2.05159200 & -4.17797700 & -4.38773100 \\
\hline S & -1.11772700 & -4.26257900 & -2.86585200 \\
\hline $\mathrm{C}$ & -2.52881500 & -5.49380400 & -4.98429000 \\
\hline $\mathrm{C}$ & -3.46115600 & -5.23721600 & -6.18067700 \\
\hline $\mathrm{C}$ & 3.69933600 & -2.55114400 & -0.20037500 \\
\hline $\mathrm{O}$ & 4.89204400 & -2.57114300 & -1.02468300 \\
\hline $\mathrm{C}$ & 2.54118400 & -2.13853900 & -1.06371000 \\
\hline S & 0.99003900 & -2.93815800 & -0.75780700 \\
\hline 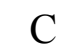 & 2.73911500 & -1.36482600 & -2.16864800 \\
\hline
\end{tabular}




\begin{tabular}{|c|c|c|c|}
\hline $\mathrm{S}$ & 1.40598000 & -1.00867400 & -3.27893700 \\
\hline $\mathrm{C}$ & 4.10116100 & -0.83138800 & -2.58368700 \\
\hline $\mathrm{C}$ & 5.18678800 & -1.25551300 & -1.57651300 \\
\hline W & -0.50851900 & -2.04090300 & -2.31445900 \\
\hline $\mathrm{H}$ & -1.65455500 & -6.07860700 & -5.30940800 \\
\hline $\mathrm{H}$ & -3.05035000 & -6.09775100 & -4.22546400 \\
\hline $\mathrm{H}$ & -4.46571900 & -4.94096800 & -5.82533200 \\
\hline $\mathrm{H}$ & -3.55648600 & -6.12151200 & -6.82192400 \\
\hline $\mathrm{H}$ & -2.36289900 & -2.21712300 & -7.02709100 \\
\hline $\mathrm{H}$ & -3.95471700 & -2.52288700 & -6.27757500 \\
\hline $\mathrm{H}$ & 3.84930300 & -1.87455300 & 0.65905100 \\
\hline $\mathrm{H}$ & 3.56885000 & -3.57206800 & 0.17311000 \\
\hline $\mathrm{H}$ & 5.25936100 & -0.53072400 & -0.75651500 \\
\hline $\mathrm{H}$ & 6.16840100 & -1.35020000 & -2.05591100 \\
\hline $\mathrm{H}$ & 4.06220500 & 0.26378300 & -2.65728300 \\
\hline $\mathrm{H}$ & 4.33102100 & -1.22140400 & -3.58731300 \\
\hline $\mathrm{H}$ & -4.46602200 & -1.86663600 & -1.36981600 \\
\hline $\mathrm{H}$ & -3.61037500 & -3.30433000 & -1.98544400 \\
\hline $\mathrm{H}$ & -6.66823600 & -3.97181600 & -0.52296700 \\
\hline $\mathrm{H}$ & -5.61641000 & -4.60622000 & -1.71551000 \\
\hline $\mathrm{H}$ & -3.89444100 & -4.38219800 & 0.27802100 \\
\hline $\mathrm{H}$ & -5.95347100 & 9.21098100 & -1.05493100 \\
\hline $\mathrm{H}$ & -5.45591000 & 10.07980400 & 0.28652000 \\
\hline $\mathrm{H}$ & -7.36742000 & 7.82054600 & 0.20410100 \\
\hline $\mathrm{H}$ & -4.71479800 & 8.09632800 & 1.72002200 \\
\hline $\mathrm{H}$ & -5.94352900 & 6.94188700 & 2.18911600 \\
\hline $\mathrm{H}$ & -4.96354600 & 4.56207900 & -2.30400900 \\
\hline $\mathrm{H}$ & -6.49913300 & 6.33284000 & -1.33314600 \\
\hline $\mathrm{H}$ & -2.78309800 & 6.38991000 & 2.31848400 \\
\hline $\mathrm{H}$ & -0.85346500 & 4.83421900 & 2.04302700 \\
\hline $\mathrm{H}$ & -0.71361800 & 3.29438300 & 0.08635000 \\
\hline $\mathrm{H}$ & -2.48750100 & 3.27974500 & -1.64556100 \\
\hline $\mathrm{H}$ & 0.53316900 & -0.16378600 & 9.12838800 \\
\hline $\mathrm{H}$ & 1.92368800 & -0.49331400 & 8.18354500 \\
\hline $\mathrm{H}$ & 0.40644300 & -2.86290300 & 8.68704500 \\
\hline $\mathrm{H}$ & -1.04238000 & -0.88122600 & 6.86123900 \\
\hline $\mathrm{H}$ & -1.50274400 & -1.20887700 & 8.53407800 \\
\hline $\mathrm{H}$ & -1.02639900 & -1.96980500 & 4.45991900 \\
\hline $\mathrm{H}$ & -1.69901600 & -3.24432800 & 2.44240500 \\
\hline $\mathrm{H}$ & -3.04765200 & -5.31057700 & 2.62815300 \\
\hline $\mathrm{H}$ & -3.80735500 & -6.16337600 & 4.85373400 \\
\hline $\mathrm{H}$ & -3.65994700 & -5.51230500 & 7.64072300 \\
\hline $\mathrm{H}$ & -2.60495200 & -3.72246100 & 9.11545300 \\
\hline $\mathrm{H}$ & 2.79702000 & 0.02123400 & 3.71900100 \\
\hline $\mathrm{H}$ & 5.03865000 & -0.36490100 & 4.70911100 \\
\hline $\mathrm{H}$ & 7.11488800 & 0.27419000 & 3.47191300 \\
\hline $\mathrm{H}$ & 2.54765200 & 1.08539500 & 1.46957900 \\
\hline $\mathrm{H}$ & 4.08005300 & 2.06817500 & -0.71000500 \\
\hline $\mathrm{H}$ & 6.54401000 & 2.42418900 & -1.24061300 \\
\hline 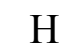 & 9.16340400 & 1.47944900 & -2.00732700 \\
\hline
\end{tabular}




$\begin{array}{cccc}\mathrm{H} & 10.30399600 & 0.72317900 & -1.04270400 \\ \mathrm{H} & 8.66644800 & 3.11375100 & -0.43775500 \\ \mathrm{H} & 8.80450700 & 0.39491200 & 0.86145900 \\ \mathrm{H} & 8.73134900 & 1.77176200 & 1.94987700 \\ \mathrm{H} & 0.08304200 & 3.74150100 & -2.66095100 \\ \mathrm{H} & 0.89956800 & 2.82224200 & -3.93851600 \\ \mathrm{H} & 3.69271700 & 4.95152400 & -4.53662700 \\ \mathrm{H} & 3.54733500 & 3.39349000 & -3.87182700 \\ \mathrm{H} & 1.34423400 & 5.36615500 & -3.91736900 \\ \mathrm{H} & -8.12681500 & 9.88073300 & 1.42804200 \\ \mathrm{H} & 2.98959500 & 4.69136700 & -1.36739000 \\ \mathrm{H} & 11.19724300 & 3.40210800 & -0.12587900 \\ \mathrm{H} & 0.85571200 & -3.25508100 & 6.24797600 \\ \mathrm{O} & -0.89150900 & -0.00801500 & -1.57648700 \\ \mathrm{H} & -0.18516400 & 0.72631300 & -1.49369900 \\ \mathrm{H} & -1.55754700 & -0.03961400 & -0.84961100 \\ \mathrm{H} & -4.34838700 & -2.36472700 & 1.66161100 \\ \mathrm{C} & -0.00400700 & -0.33772100 & 2.08168700 \\ \mathrm{C} & -0.27297100 & 0.44839800 & 2.97951300 \\ \mathrm{H} & 0.23795200 & -1.02432000 & 1.29058700 \\ \mathrm{H} & -0.51109000 & 1.16295100 & 3.73466700\end{array}$

\begin{tabular}{lrrr}
\multicolumn{5}{l}{$\mathbf{L N}-\mathbf{T S}, E=-3328.294434$} \\
$\mathrm{~N}$ & 2.04148200 & 5.65056300 & -3.13740600 \\
$\mathrm{C}$ & 0.98535600 & 5.70075500 & -2.09965100 \\
$\mathrm{C}$ & 1.48422700 & 6.08126400 & -0.67945800 \\
$\mathrm{O}$ & 0.92700400 & 6.95564500 & 0.01716400 \\
$\mathrm{C}$ & 0.22405000 & 4.35106800 & -2.05457300 \\
$\mathrm{C}$ & 0.80309900 & 3.32912600 & -1.07170200 \\
$\mathrm{O}$ & 2.03325800 & 3.29770400 & -0.77494000 \\
$\mathrm{O}$ & -0.13998200 & 2.55477600 & -0.56442000 \\
$\mathrm{~N}$ & -4.93159900 & -4.92988200 & -1.36894200 \\
$\mathrm{C}$ & -3.82445200 & -4.35002400 & -0.61607600 \\
$\mathrm{C}$ & -4.27795900 & -3.92962500 & 0.76335300 \\
$\mathrm{O}$ & -5.45947800 & -4.01819700 & 1.17132100 \\
$\mathrm{C}$ & -3.14641800 & -3.13818900 & -1.33810300 \\
$\mathrm{~S}$ & -1.41369900 & -2.91908700 & -0.74122900 \\
$\mathrm{~N}$ & 9.43007000 & 3.64138000 & -1.33034600 \\
$\mathrm{C}$ & 8.89806900 & 3.91186700 & 0.03357600 \\
$\mathrm{C}$ & 10.02469700 & 4.61606600 & 0.79551800 \\
$\mathrm{O}$ & 10.30654100 & 4.47076900 & 1.99862900 \\
$\mathrm{C}$ & 8.30819700 & 2.72123000 & 0.83595300 \\
$\mathrm{C}$ & 6.80259200 & 2.57024700 & 0.84829000 \\
$\mathrm{C}$ & 5.81752400 & 3.17476400 & 0.07197800 \\
$\mathrm{C}$ & 6.10981300 & 1.65631200 & 1.73633700 \\
$\mathrm{~N}$ & 4.55639500 & 2.69148000 & 0.42772300 \\
$\mathrm{C}$ & 4.71224700 & 1.74833100 & 1.43928100 \\
$\mathrm{C}$ & 6.54625400 & 0.76039100 & 2.74389900 \\
$\mathrm{C}$ & 3.75299400 & 0.95082200 & 2.09680000 \\
$\mathrm{C}$ & 5.59429900 & -0.02079600 & 3.41202900 \\
& & & $\mathrm{~S} 30$ \\
& & &
\end{tabular}




\begin{tabular}{|c|c|c|c|}
\hline $\mathrm{C}$ & 4.21078600 & 0.06804400 & 3.08485100 \\
\hline $\mathrm{N}$ & 2.29458600 & -2.67070000 & 8.19675300 \\
\hline $\mathrm{C}$ & 1.76368000 & -3.71717000 & 7.33101000 \\
\hline$C$ & 2.44947300 & -3.65078400 & 5.97397500 \\
\hline $\mathrm{O}$ & 3.24481300 & -2.74090500 & 5.66317900 \\
\hline $\mathrm{C}$ & 0.19712100 & -3.64948100 & 7.15383500 \\
\hline $\mathrm{C}$ & -0.40997200 & -4.83090500 & 6.44058000 \\
\hline $\mathrm{C}$ & -0.87923400 & -5.99401800 & 7.03372600 \\
\hline C & -0.60008300 & -4.98872900 & 5.00609100 \\
\hline $\mathrm{N}$ & -1.34521100 & -6.87229000 & 6.04869700 \\
\hline $\mathrm{C}$ & -1.18543300 & -6.27538200 & 4.79238600 \\
\hline $\mathrm{C}$ & -0.33949200 & -4.15814800 & 3.88626500 \\
\hline $\mathrm{C}$ & -1.50806900 & -6.75277200 & 3.50699300 \\
\hline $\mathrm{C}$ & -0.65836800 & -4.62278500 & 2.60431600 \\
\hline $\mathrm{C}$ & -1.23625800 & -5.91003900 & 2.41963000 \\
\hline $\mathrm{N}$ & -7.43371000 & 8.08790200 & 2.83907600 \\
\hline C & -7.87454100 & 6.75987300 & 3.30116000 \\
\hline $\mathrm{C}$ & -8.37426900 & 7.02357500 & 4.70639800 \\
\hline $\mathrm{O}$ & -8.20036600 & 6.28549200 & 5.69909000 \\
\hline $\mathrm{C}$ & -6.85882300 & 5.59917100 & 3.21565300 \\
\hline $\mathrm{C}$ & -6.58998600 & 5.09610700 & 1.81584000 \\
\hline $\mathrm{C}$ & -7.27528000 & 5.38250700 & 0.64311300 \\
\hline $\mathrm{C}$ & -5.55073900 & 4.14127300 & 1.46370300 \\
\hline $\mathrm{N}$ & -6.71772000 & 4.65524600 & -0.41938700 \\
\hline $\mathrm{C}$ & -5.65596900 & 3.88229000 & 0.06230400 \\
\hline $\mathrm{C}$ & -4.54489900 & 3.47665200 & 2.20924700 \\
\hline $\mathrm{C}$ & -4.79817000 & 2.98658200 & -0.60793500 \\
\hline $\mathrm{C}$ & -3.68744700 & 2.58850500 & 1.54763200 \\
\hline $\mathrm{C}$ & -3.81095400 & 2.34193100 & 0.15064400 \\
\hline $\mathrm{C}$ & -2.95256700 & -1.64200700 & -6.60384900 \\
\hline $\mathrm{O}$ & -2.83932700 & -2.72089400 & -7.57504900 \\
\hline $\mathrm{C}$ & -2.09238500 & -1.92628700 & -5.40243600 \\
\hline $\mathrm{S}$ & -1.56525700 & -0.52769300 & -4.44531300 \\
\hline $\mathrm{C}$ & -1.62461200 & -3.18178100 & -5.14619200 \\
\hline $\mathrm{S}$ & -0.49093000 & -3.46470100 & -3.81366300 \\
\hline $\mathrm{C}$ & -1.97674500 & -4.38700100 & -6.00586700 \\
\hline $\mathrm{C}$ & -3.10301400 & -4.02533400 & -6.98238400 \\
\hline $\mathrm{C}$ & 4.13396600 & -1.70394200 & -1.00661900 \\
\hline $\mathrm{O}$ & 5.22560800 & -1.27432300 & -1.85773800 \\
\hline $\mathrm{C}$ & 2.84424500 & -1.25422900 & -1.62973400 \\
\hline $\mathrm{S}$ & 1.52364000 & -2.42349900 & -1.55278400 \\
\hline $\mathrm{C}$ & 2.77931900 & -0.13043200 & -2.39567500 \\
\hline $\mathrm{S}$ & 1.27552800 & 0.31537300 & -3.24650400 \\
\hline $\mathrm{C}$ & 4.00574600 & 0.71449200 & -2.70799000 \\
\hline $\mathrm{C}$ & 5.25862400 & 0.17740100 & -1.98065400 \\
\hline W & -0.29815400 & -1.40130000 & -2.62108500 \\
\hline $\mathrm{H}$ & -1.08408200 & -4.70850900 & -6.56439800 \\
\hline $\mathrm{H}$ & -2.27830100 & -5.23439600 & -5.37103500 \\
\hline $\mathrm{H}$ & -4.07642100 & -4.00442200 & -6.45772700 \\
\hline $\mathrm{H}$ & 16600300 & -4.72706700 & -7.8230 \\
\hline
\end{tabular}




\begin{tabular}{|c|c|c|c|}
\hline $\mathrm{H}$ & -2.61291900 & -0.73849100 & -7.12241800 \\
\hline $\mathrm{H}$ & -4.01758200 & -1.50655000 & -6.33053400 \\
\hline $\mathrm{H}$ & 4.27627900 & -1.30344900 & 0.01311000 \\
\hline $\mathrm{H}$ & 4.18978300 & -2.79627400 & -0.96739800 \\
\hline $\mathrm{H}$ & 5.33735000 & 0.61653500 & -0.97694200 \\
\hline $\mathrm{H}$ & 6.17440100 & 0.40453200 & -2.54015600 \\
\hline $\mathrm{H}$ & 3.83672300 & 1.76387300 & -2.42261100 \\
\hline $\mathrm{H}$ & 4.15840200 & 0.69900700 & -3.79879500 \\
\hline $\mathrm{H}$ & -3.73869300 & -2.21930400 & -1.22539300 \\
\hline $\mathrm{H}$ & -3.09342900 & -3.38304700 & -2.40678300 \\
\hline $\mathrm{H}$ & -5.84598200 & -4.75787700 & -0.95796300 \\
\hline $\mathrm{H}$ & -4.88283000 & -4.77785700 & -2.37028900 \\
\hline $\mathrm{H}$ & -3.01580200 & -5.08780500 & -0.47315100 \\
\hline $\mathrm{H}$ & -7.31415300 & 8.14842500 & 1.82918900 \\
\hline $\mathrm{H}$ & -6.58837300 & 8.40903800 & 3.31145800 \\
\hline $\mathrm{H}$ & -8.76860800 & 6.49753100 & 2.70971900 \\
\hline $\mathrm{H}$ & -5.91626000 & 5.92458400 & 3.68327700 \\
\hline $\mathrm{H}$ & -7.22735200 & 4.77644800 & 3.84608300 \\
\hline $\mathrm{H}$ & -7.03013400 & 4.68256500 & -1.37850400 \\
\hline $\mathrm{H}$ & -8.12351300 & 6.03168600 & 0.47357700 \\
\hline $\mathrm{H}$ & -4.43901800 & 3.65033200 & 3.27832200 \\
\hline $\mathrm{H}$ & -2.90368100 & 2.06986600 & 2.09274800 \\
\hline $\mathrm{H}$ & -3.12700500 & 1.64336200 & -0.32480800 \\
\hline $\mathrm{H}$ & -4.88818100 & 2.79291600 & -1.67429000 \\
\hline $\mathrm{H}$ & 1.64920300 & -2.27662000 & 8.87090500 \\
\hline $\mathrm{H}$ & 2.88117600 & -1.99309700 & 7.71625200 \\
\hline $\mathrm{H}$ & 1.97966500 & -4.72358400 & 7.73300100 \\
\hline $\mathrm{H}$ & -0.05223500 & -2.71121000 & 6.64016300 \\
\hline $\mathrm{H}$ & -0.21832100 & -3.57703200 & 8.16981700 \\
\hline $\mathrm{H}$ & 0.09237000 & -3.16721800 & 4.00883000 \\
\hline $\mathrm{H}$ & -0.48476800 & -4.00374100 & 1.72784800 \\
\hline $\mathrm{H}$ & -1.47053800 & -6.23265100 & 1.40838900 \\
\hline $\mathrm{H}$ & -1.95878200 & -7.73172500 & 3.35708200 \\
\hline $\mathrm{H}$ & -1.76212200 & -7.77585400 & 6.21612700 \\
\hline $\mathrm{H}$ & -0.92227600 & -6.25678100 & 8.08182200 \\
\hline $\mathrm{H}$ & 3.50709100 & -0.58150200 & 3.59995800 \\
\hline $\mathrm{H}$ & 5.90569700 & -0.72418900 & 4.18130500 \\
\hline $\mathrm{H}$ & 7.60311600 & 0.66992400 & 2.98939600 \\
\hline $\mathrm{H}$ & 2.70484400 & 0.99108200 & 1.81640800 \\
\hline $\mathrm{H}$ & 3.66152200 & 2.91683000 & -0.02179700 \\
\hline $\mathrm{H}$ & 5.91404100 & 3.90474800 & -0.72045300 \\
\hline $\mathrm{H}$ & 8.70460700 & 3.57672500 & -2.04136600 \\
\hline $\mathrm{H}$ & 10.03686700 & 2.82269300 & -1.3611220 \\
\hline $\mathrm{H}$ & 8.12295000 & 4.68783100 & -0.08191100 \\
\hline $\mathrm{H}$ & 8.76819500 & 1.79348400 & 0.45567100 \\
\hline $\mathrm{H}$ & 8.65406100 & 2.82010400 & 1.87509600 \\
\hline $\mathrm{H}$ & -0.81982700 & 4.50934100 & -1.77184600 \\
\hline $\mathrm{H}$ & 0.22983000 & 3.90665700 & -3.05882300 \\
\hline 11 & 2.60404100 & 6.49379100 & -3.2246490 \\
\hline 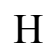 & 2.59959400 & 4.79900800 & 3.1081650 \\
\hline
\end{tabular}




$\begin{array}{cccc}\mathrm{H} & 0.26525400 & 6.47939800 & -2.38376200 \\ \mathrm{H} & -8.92328800 & 7.98196700 & 4.79282600 \\ \mathrm{H} & 2.39761800 & 5.56826900 & -0.33705700 \\ \mathrm{H} & 10.60741900 & 5.30303900 & 0.14847300 \\ \mathrm{H} & 2.20934800 & -4.46048900 & 5.26726700 \\ \mathrm{O} & -1.12610000 & -0.05226100 & -1.29203600 \\ \mathrm{H} & 0.02571300 & 1.78799900 & 0.28748100 \\ \mathrm{H} & -0.92635000 & 0.88701000 & -1.49984700 \\ \mathrm{H} & -3.48388800 & -3.50476200 & 1.40021100 \\ \mathrm{C} & -0.66551800 & -0.20555900 & 0.86616600 \\ \mathrm{C} & -0.09784400 & 0.82250100 & 1.33921800 \\ \mathrm{H} & -1.10314000 & -1.16386700 & 0.58553800 \\ \mathrm{H} & 0.28443400 & 1.16396300 & 2.29003300\end{array}$

LN-EP1, $E=-3328.403882$

$\begin{array}{lccc}\mathrm{N} & 2.00132300 & 3.11600400 & -5.30696000 \\ \mathrm{C} & 0.92430800 & 3.79580400 & -4.54070900 \\ \mathrm{C} & 1.41703300 & 4.73318500 & -3.41463800 \\ \mathrm{O} & 1.15570300 & 5.95733600 & -3.39255200 \\ \mathrm{C} & 0.00193200 & 2.72632300 & -3.93039500 \\ \mathrm{C} & 0.63857300 & 1.93178500 & -2.77138900 \\ \mathrm{O} & 1.91282900 & 1.95413100 & -2.60283000 \\ \mathrm{O} & -0.23009500 & 1.30866600 & -2.04363700 \\ \mathrm{~N} & -4.78923400 & -5.20322600 & 1.64831900 \\ \mathrm{C} & -3.82868300 & -4.09080400 & 1.61291900 \\ \mathrm{C} & -4.35015700 & -2.94447900 & 2.45773400 \\ \mathrm{O} & -5.47899200 & -2.94376900 & 3.00335600 \\ \mathrm{C} & -3.47663900 & -3.57497900 & 0.16972800 \\ \mathrm{~S} & -2.20845500 & -2.20794200 & 0.18489600 \\ \mathrm{~N} & 9.41132700 & 2.46217900 & -2.74612700 \\ \mathrm{C} & 8.82800600 & 3.45023900 & -1.79883200 \\ \mathrm{C} & 9.97126100 & 4.39935600 & -1.42071600 \\ \mathrm{O} & 10.14911700 & 4.93775100 & -0.31314200 \\ \mathrm{C} & 8.09405400 & 2.90836000 & -0.54536200 \\ \mathrm{C} & 6.59359400 & 2.72334900 & -0.61849100 \\ \mathrm{C} & 5.71323700 & 2.67319400 & -1.69774400 \\ \mathrm{C} & 5.78799700 & 2.50489900 & 0.56743500 \\ \mathrm{~N} & 4.40968600 & 2.44259400 & -1.24881600 \\ \mathrm{C} & 4.43522200 & 2.32347900 & 0.13793800 \\ \mathrm{C} & 6.09363000 & 2.43121800 & 1.94946900 \\ \mathrm{C} & 3.39162000 & 2.04770200 & 1.04308800 \\ \mathrm{C} & 5.05806700 & 2.17020400 & 2.85646800 \\ \mathrm{C} & 3.72199300 & 1.97519900 & 2.40295100 \\ \mathrm{~N} & 2.31911400 & 1.77738700 & 8.68867400 \\ \mathrm{C} & 1.57336300 & 0.65564500 & 8.12125100 \\ \mathrm{C} & 2.50940100 & -0.19917900 & 7.28074200 \\ \mathrm{O} & 3.67625800 & 0.14826600 & 7.00334700 \\ \mathrm{C} & 0.32354700 & 1.08186800 & 7.25161400 \\ \mathrm{C} & -0.59882700 & -0.05520000 & 6.88900600 \\ \mathrm{C} & -1.76941700 & -0.39125900 & 7.55420600 \\ & & & \mathrm{~S} 33 \\ \mathrm{~N} & & & \\ \mathrm{C} & & & \\ \mathrm{C} & & & \end{array}$




\begin{tabular}{|c|c|c|c|}
\hline & -0.43380400 & -1.03154700 & 5.82086400 \\
\hline $\mathrm{N}$ & -2.33566900 & -1.52948900 & 6.97273500 \\
\hline $\mathrm{C}$ & -1.53206800 & -1.94309800 & 5.90515300 \\
\hline $\mathrm{C}$ & 0.52581000 & -1.21392400 & 4.79166500 \\
\hline C & -1.68646700 & -3.02702700 & 5.01910500 \\
\hline $\mathrm{C}$ & 0.38179800 & -2.29034100 & 3.90872700 \\
\hline $\mathrm{C}$ & -0.71323000 & -3.19003900 & 4.02403200 \\
\hline $\mathrm{N}$ & -7.56359100 & 8.08094400 & -1.39543500 \\
\hline $\mathrm{C}$ & -8.01068700 & 7.16826600 & -0.33014300 \\
\hline $\mathrm{C}$ & -8.49894500 & 8.10424500 & 0.75605300 \\
\hline $\mathrm{O}$ & -8.25915700 & 8.00280400 & 1.97389100 \\
\hline $\mathrm{C}$ & -6.99775500 & 6.11286700 & 0.16347600 \\
\hline $\mathrm{C}$ & -6.63196300 & 5.08166100 & -0.87873600 \\
\hline $\mathrm{C}$ & -7.37931800 & 4.65110900 & -1.96725200 \\
\hline $\mathrm{C}$ & -5.40492400 & 4.30411500 & -0.88476400 \\
\hline $\mathrm{N}$ & -6.67762000 & 3.64776400 & -2.65136700 \\
\hline $\mathrm{C}$ & -5.45968500 & 3.41651100 & -2.00312000 \\
\hline $\mathrm{C}$ & -4.26826700 & 4.27286400 & -0.03855200 \\
\hline $\mathrm{C}$ & -4.41979900 & 2.51371600 & -2.30242800 \\
\hline $\mathrm{C}$ & -3.23413300 & 3.37583000 & -0.33111600 \\
\hline $\mathrm{C}$ & -3.30318100 & 2.50320800 & -1.45495500 \\
\hline $\mathrm{C}$ & -2.80285700 & -5.00397300 & -4.58556200 \\
\hline $\mathrm{O}$ & -2.68957800 & -6.44192000 & -4.81929900 \\
\hline $\mathrm{C}$ & -2.02177000 & -4.60018100 & -3.36037300 \\
\hline $\mathrm{S}$ & -1.40293400 & -2.93108600 & -3.31580800 \\
\hline $\mathrm{C}$ & -1.65988600 & -5.51891700 & -2.42207000 \\
\hline $\mathrm{S}$ & -0.50501900 & -5.04695000 & -1.14019900 \\
\hline $\mathrm{C}$ & -2.04694300 & -6.98647300 & -2.50620500 \\
\hline $\mathrm{C}$ & -3.06603600 & -7.20841400 & -3.63695400 \\
\hline $\mathrm{C}$ & 4.21323300 & -2.05363800 & 0.29091900 \\
\hline $\mathrm{O}$ & 5.30240300 & -2.11272200 & -0.67084500 \\
\hline $\mathrm{C}$ & 2.91969200 & -2.07604700 & -0.47032800 \\
\hline $\mathrm{S}$ & 1.62155200 & -3.11067500 & 0.17660200 \\
\hline $\mathrm{C}$ & 2.86544400 & -1.59962200 & -1.74461600 \\
\hline $\mathrm{S}$ & 1.44535000 & -1.88733400 & -2.77303000 \\
\hline $\mathrm{C}$ & 4.04790400 & -0.95216200 & -2.44673300 \\
\hline $\mathrm{C}$ & 5.29565300 & -0.94513700 & -1.54259400 \\
\hline W & -0.21194600 & -2.72209400 & -1.24650200 \\
\hline $\mathrm{H}$ & -1.14276000 & -7.58654600 & -2.69153800 \\
\hline $\mathrm{H}$ & -2.46702600 & -7.33582500 & -1.54904400 \\
\hline $\mathrm{H}$ & -4.07664500 & -6.90269300 & -3.30753800 \\
\hline $\mathrm{H}$ & -3.09832500 & -8.25579900 & -3.96018400 \\
\hline $\mathrm{H}$ & -2.38984100 & -4.52526100 & -5.47969300 \\
\hline $\mathrm{H}$ & -3.87277900 & -4.73185500 & -4.50478300 \\
\hline $\mathrm{H}$ & 4.32658300 & -1.15532100 & 0.92440400 \\
\hline $\mathrm{H}$ & 4.31346400 & -2.94561500 & 0.91858400 \\
\hline $\mathrm{H}$ & 5.32613900 & -0.03735700 & -0.92455400 \\
\hline $\mathrm{H}$ & 6.22110900 & -1.00739400 & -2.12766200 \\
\hline $\mathrm{H}$ & 3.78527700 & 0.07028700 & -2.74894000 \\
\hline H & 4.24426500 & -1.52011300 & -3.3693 \\
\hline
\end{tabular}




\begin{tabular}{|c|c|c|c|}
\hline $\mathrm{H}$ & -4.38805300 & -3.19571900 & -0.31448100 \\
\hline $\mathrm{H}$ & -3.09104100 & -4.41605500 & -0.41322100 \\
\hline $\mathrm{H}$ & -5.72936500 & -4.91882300 & 1.91248600 \\
\hline $\mathrm{H}$ & -4.74015500 & -5.82806600 & 0.85045100 \\
\hline $\mathrm{H}$ & -2.86648600 & -4.39435300 & 2.05569400 \\
\hline $\mathrm{H}$ & -7.47008800 & 7.62508600 & -2.30137100 \\
\hline $\mathrm{H}$ & -6.69955600 & 8.56940200 & -1.15959500 \\
\hline & -8.91284800 & 6.65756400 & -0.70764300 \\
\hline $\mathrm{H}$ & -6.08968700 & 6.63237500 & 0.50335900 \\
\hline $\mathrm{H}$ & -7.41007600 & 5.62854800 & 1.06122200 \\
\hline $\mathrm{H}$ & -7.00257100 & 3.15695100 & -3.47106700 \\
\hline $\mathrm{H}$ & -8.35871300 & 4.96126500 & -2.30583900 \\
\hline $\mathrm{H}$ & -4.20146100 & 4.92545200 & 0.82977400 \\
\hline $\mathrm{H}$ & -2.35929400 & 3.32249800 & 0.31093200 \\
\hline $\mathrm{H}$ & -2.47298900 & 1.82919800 & -1.65229800 \\
\hline $\mathrm{H}$ & -4.47243300 & 1.83593600 & -3.15127200 \\
\hline $\mathrm{H}$ & 1.78700700 & 2.62575600 & 8.84655300 \\
\hline $\mathrm{H}$ & 3.22913000 & 1.92189700 & 8.25736000 \\
\hline $\mathrm{H}$ & 1.17847100 & -0.00266600 & 8.91429100 \\
\hline $\mathrm{H}$ & 0.68543500 & 1.60450600 & 6.35527300 \\
\hline $\mathrm{H}$ & -0.22752200 & 1.82111200 & 7.85114100 \\
\hline $\mathrm{H}$ & 1.35445500 & -0.52082000 & 4.66355900 \\
\hline $\mathrm{H}$ & 1.09402800 & -2.44164500 & 3.10181700 \\
\hline $\mathrm{H}$ & -0.80078100 & -3.99759400 & 3.30326700 \\
\hline $\mathrm{H}$ & -2.53494100 & -3.70423400 & 5.08928700 \\
\hline $\mathrm{H}$ & -3.22456200 & -1.93844800 & 7.22128900 \\
\hline $\mathrm{H}$ & -2.24229800 & 0.10217800 & 8.39213800 \\
\hline $\mathrm{H}$ & 2.94051400 & 1.74721400 & 3.12460200 \\
\hline $\mathrm{H}$ & 5.27261300 & 2.09410600 & 3.92072200 \\
\hline $\mathrm{H}$ & 7.11487400 & 2.56241500 & 2.30311000 \\
\hline $\mathrm{H}$ & 2.37711800 & 1.86148500 & 0.70513700 \\
\hline $\mathrm{H}$ & 3.56668700 & 2.27244700 & -1.81420300 \\
\hline $\mathrm{H}$ & 5.91372700 & 2.77170200 & -2.75633200 \\
\hline $\mathrm{H}$ & 8.73021300 & 2.06879700 & -3.39210300 \\
\hline $\mathrm{H}$ & 9.92462100 & 1.71978000 & -2.27167500 \\
\hline $\mathrm{H}$ & 8.12975000 & 4.07407300 & -2.38238800 \\
\hline $\mathrm{H}$ & 8.56937800 & 1.95300200 & -0.26283500 \\
\hline $\mathrm{H}$ & 8.31204400 & 3.59962600 & 0.28139300 \\
\hline $\mathrm{H}$ & -0.92676900 & 3.17058500 & -3.55561000 \\
\hline $\mathrm{H}$ & -0.27010500 & 2.00710400 & -4.71458800 \\
\hline $\mathrm{H}$ & 2.66431300 & 3.75188100 & -5.74825200 \\
\hline $\mathrm{H}$ & 2.45578100 & 2.40007200 & -4.73731600 \\
\hline $\mathrm{H}$ & 0.34503800 & 4.42023800 & -5.23447900 \\
\hline $\mathrm{H}$ & -9.09849800 & 8.94721800 & 0.35812800 \\
\hline $\mathrm{H}$ & 2.04748800 & 4.26854200 & -2.64025500 \\
\hline $\mathrm{H}$ & 10.67120300 & 4.57368600 & -2.26337500 \\
\hline $\mathrm{H}$ & 2.10742700 & -1.16128400 & 6.93141500 \\
\hline $\mathrm{O}$ & -0.11486200 & -0.54820500 & -0.48325800 \\
\hline $\mathrm{H}$ & -0.23287100 & 1.99037800 & 0.54426600 \\
\hline $\mathrm{H}$ & -0.09899400 & 0.25004600 & -1.20189000 \\
\hline
\end{tabular}




$\begin{array}{lrrr}\mathrm{H} & -3.66871600 & -2.08494600 & 2.54735300 \\ \mathrm{C} & -0.03660400 & -0.12231000 & 0.82795900 \\ \mathrm{C} & -0.10136900 & 1.16530400 & 1.23772900 \\ \mathrm{H} & 0.08426900 & -0.94768000 & 1.51919600 \\ \mathrm{H} & -0.03217600 & 1.38609600 & 2.29881800\end{array}$

XE-E,$E=-5352.913179$

$\mathrm{N}$

C

C

$\mathrm{O}$

C

C

$\mathrm{O}$

$\mathrm{O}$

$\mathrm{N}$

C

C

$\mathrm{O}$

C

C

C

C

$\mathrm{N}$

C

C

$\mathrm{O}$

C

$\mathrm{N}$

C

C

$\mathrm{O}$

C

C

S

C

$\mathrm{N}$

C

C

$\mathrm{O}$

C

S

$\mathrm{N}$

C

C

$\mathrm{O}$

C

C

C

C
$-5.06377200-2.23342400$

$-3.83520000-2.08130800$

$-3.80957100-0.81665300$

$\begin{array}{ll}-2.81764900 & -0.48908300\end{array}$

$-2.51083100 \quad-2.14203000$

$-2.33125800-0.95547700$

$\begin{array}{ll}-3.05301700 & 0.06283800\end{array}$

$-1.25324700-1.14040400$

$1.77711500-7.12776200$

$0.74691000-6.63254000$

$-0.64291200 \quad-7.19931400$

$\begin{array}{ll}-1.50706900 & -7.51017500\end{array}$

$0.71583600 \quad-5.06377700$

$-0.10180100-4.44191000$

$0.22781700-4.53799800$

$\begin{array}{lll}0.54992900 & -4.58697500\end{array}$

$\begin{array}{llll}4.73806300 & -6.53589400 & -4.29880400\end{array}$

$\begin{array}{llll}4.25154400 & -5.60569900 & -5.35047300\end{array}$

$\begin{array}{llll}4.45246500 & -4.14376000 & -4.86580300\end{array}$

$\begin{array}{llll}3.91891900 & -3.15582300 & -5.44163100\end{array}$

$\begin{array}{lll}2.81738700 & -5.89155600 & -5.83213100\end{array}$

$\begin{array}{llll}5.29472300 & -4.01354400 & -3.77710700\end{array}$

$\begin{array}{llll}5.66351900 & -2.70630100 & -3.20614400\end{array}$

$\begin{array}{lll}7.07875500 & -2.33353700 & -3.64293000\end{array}$

$\begin{array}{llll}7.87957900 & -1.64975500 & -2.98070100\end{array}$

$\begin{array}{llll}5.39786300 & -2.53229900 & -1.68624500\end{array}$

$\begin{array}{llll}6.31299700 & -3.32761400 & -0.73685400\end{array}$

$\begin{array}{llll}5.99189500 & -3.03502500 & 1.07126900\end{array}$

$\begin{array}{rrrr}6.55492800 & -1.28236300 & 1.22946200\end{array}$

$\begin{array}{llll}4.69836500 & 0.48142000 & -4.37316500\end{array}$

$\begin{array}{llll}3.86407300 & 1.37470400 & -3.55097100\end{array}$

$\begin{array}{llll}4.42869600 & 1.40288700 & -2.09224700\end{array}$

$\begin{array}{llll}4.31258100 & 0.41327500 & -1.30320300\end{array}$

$\begin{array}{llll}2.42091800 & 0.84721600 & -3.52332400\end{array}$

$\begin{array}{llll}1.27635100 & 1.90586800 & -2.50182600\end{array}$

$\begin{array}{llll}5.09194900 & 2.53719200 & -1.71156800\end{array}$

$\begin{array}{llll}5.69364800 & 2.80685300 & -0.37374400\end{array}$

$\begin{array}{llll}6.98071400 & 1.98264700 & -0.20353100\end{array}$

$\begin{array}{llll}7.96807700 & 2.37795400 & 0.44124700\end{array}$

$\begin{array}{llll}4.70883200 & 2.62186900 & 0.84327100\end{array}$

$\begin{array}{lll}3.39395700 & 3.40315500 & 0.58331600\end{array}$

$\begin{array}{llll}5.39151300 & 3.08388600 & 2.15184300\end{array}$

$\begin{array}{lll}2.32738900 & 3.19581900 & 1.67996200\end{array}$

S36 


$\begin{array}{lccc}\mathrm{N} & -10.25415800 & 3.54711600 & 1.85031600 \\ \mathrm{C} & -9.48130600 & 4.04287100 & 3.01790700 \\ \mathrm{C} & -10.42811500 & 4.89617500 & 3.88313900 \\ \mathrm{O} & -10.08771800 & 5.85317900 & 4.60025900 \\ \mathrm{C} & -8.15471000 & 4.79479600 & 2.73119000 \\ \mathrm{C} & -6.92211100 & 3.96438500 & 2.44142600 \\ \mathrm{C} & -6.66702300 & 2.61281200 & 2.64776600 \\ \mathrm{C} & -5.68251900 & 4.53992200 & 1.94571900 \\ \mathrm{~N} & -5.33966300 & 2.31830700 & 2.30247000 \\ \mathrm{C} & -4.71782100 & 3.48723500 & 1.86898700 \\ \mathrm{C} & -5.30212300 & 5.85090100 & 1.56519500 \\ \mathrm{C} & -3.40306700 & 3.69851900 & 1.41054300 \\ \mathrm{C} & -3.99323300 & 6.07223900 & 1.11584000 \\ \mathrm{C} & -3.05570300 & 5.00326600 & 1.03499000 \\ \mathrm{~N} & 1.23238600 & 9.49297200 & 2.55773600 \\ \mathrm{C} & 1.67842800 & 9.42122400 & 1.16081600 \\ \mathrm{C} & 0.54909100 & 9.15507100 & 0.15433100 \\ \mathrm{O} & -0.65588700 & 9.12034500 & 0.46921300 \\ \mathrm{C} & 2.82755200 & 8.37159600 & 1.02236100 \\ \mathrm{C} & 3.55379700 & 8.38253700 & -0.30194500 \\ \mathrm{C} & 4.55855800 & 9.26215800 & -0.67659100 \\ \mathrm{C} & 3.35963000 & 7.47734300 & -1.42423700 \\ \mathrm{~N} & 4.99898900 & 8.95978800 & -1.97005900 \\ \mathrm{C} & 4.28035300 & 7.86290700 & -2.45154300 \\ \mathrm{C} & 2.50470300 & 6.37096500 & -1.65160500 \\ \mathrm{C} & -8.72705700 & -5.11759800 & -1.63010100 \\ \mathrm{C} & -7.32820600 & -4.87762000 & -1.06993000 \\ \mathrm{C} & -6.69362000 & -3.61685400 & -1.15694200 \\ \mathrm{C} & 2.36423200 & 7.18312900 & -3.68194600 \\ \mathrm{C} & -10.283172300 & 5.68513200 & -2.87178700 \\ \mathrm{C} & 3.50349700 & 6.09079200 & -3.87757900 \\ \mathrm{~N} & 4.78438000 & -3.15190300 & 8.86652700 \\ \mathrm{C} & 5.53698700 & -2.17138800 & 8.06921000 \\ \mathrm{C} & 6.59905000 & -1.67418300 & 9.03280800 \\ \mathrm{O} & 6.92539800 & -0.48640500 & 9.21278500 \\ \mathrm{C} & 4.74256700 & -1.02059000 & 7.40706300 \\ \mathrm{C} & 4.13990600 & -1.32878600 & 6.05587500 \\ \mathrm{C} & 4.69832700 & -2.03818100 & 4.99929700 \\ \mathrm{C} & 2.89130300 & -0.77817700 & 5.56343300 \\ \mathrm{~N} & 3.86549300 & -1.95394000 & 3.87564700 \\ \mathrm{C} & 2.75309700 & -1.17463400 & 4.19503500 \\ \mathrm{C} & 1.88052500 & 0.01703900 & 6.15818000 \\ \mathrm{C} & 1.65819700 & -0.77453200 & 3.40387500 \\ \mathrm{C} & 0.77559200 & 0.39586800 & 5.38652800 \\ \mathrm{C} & 0.67449100 & 0.01234600 & 4.02051400 \\ \mathrm{C} & -4.90402800 & -3.56948200 \\ \mathrm{C} & -7.51305100 & -5.07829500 & -4.08744200 \\ \mathrm{C} & & -45053400\end{array}$




\begin{tabular}{|c|c|c|c|}
\hline $\mathrm{C}$ & -5.39076000 & -3.42286600 & -0.65942600 \\
\hline $\mathrm{C}$ & -5.33289000 & -5.74533900 & 0.06562800 \\
\hline $\mathrm{C}$ & -4.70611100 & -4.48892200 & -0.04294200 \\
\hline $\mathrm{C}$ & 0.21825700 & -3.78318200 & -4.66667600 \\
\hline $\mathrm{O}$ & 0.09270800 & -4.00669900 & -6.08840400 \\
\hline $\mathrm{C}$ & -0.10105400 & -2.35614300 & -4.32506900 \\
\hline $\mathrm{S}$ & -0.43852400 & -1.99921900 & -2.61884900 \\
\hline $\mathrm{C}$ & -0.21112200 & -1.37820500 & -5.26163300 \\
\hline $\mathrm{S}$ & -0.60763800 & 0.28810300 & -4.78867200 \\
\hline $\mathrm{C}$ & -0.00381700 & -1.64958300 & -6.74145700 \\
\hline $\mathrm{C}$ & 0.73995900 & -2.98051300 & -6.90963100 \\
\hline $\mathrm{C}$ & -4.54276900 & 3.38525700 & -2.54770300 \\
\hline $\mathrm{O}$ & -5.94872800 & 2.95849000 & -2.54042600 \\
\hline $\mathrm{C}$ & -3.58768500 & 2.23399100 & -2.26487100 \\
\hline $\mathrm{S}$ & -1.93199900 & 2.36675400 & -2.87830900 \\
\hline $\mathrm{C}$ & -4.00587100 & 1.08113500 & -1.66886300 \\
\hline $\mathrm{S}$ & -2.86759000 & -0.26844300 & -1.43223500 \\
\hline $\mathrm{C}$ & -5.46371900 & 0.86414000 & -1.28578400 \\
\hline $\mathrm{C}$ & -6.25792700 & 2.18803800 & -1.33390500 \\
\hline $\mathrm{W}$ & -0.75993300 & 0.32263600 & -2.44553000 \\
\hline $\mathrm{O}$ & -0.75542800 & -3.60277900 & 0.33794700 \\
\hline $\mathrm{O}$ & 3.21680300 & -5.41173900 & -1.99053000 \\
\hline $\mathrm{O}$ & 2.24845800 & -0.98906100 & -0.22682700 \\
\hline $\mathrm{O}$ & 1.63767900 & -3.55283200 & -0.75129800 \\
\hline $\mathrm{O}$ & 0.11772800 & 0.22934000 & -0.29302900 \\
\hline $\mathrm{H}$ & -7.77777100 & -4.39859100 & -4.94312000 \\
\hline $\mathrm{H}$ & -7.09148200 & -6.91893900 & -0.39284100 \\
\hline $\mathrm{H}$ & -4.80744700 & -6.57762100 & 0.52906400 \\
\hline $\mathrm{H}$ & -3.69219200 & -4.34979700 & 0.32645100 \\
\hline $\mathrm{H}$ & -4.89921100 & -2.45938700 & -0.77822500 \\
\hline $\mathrm{H}$ & -7.20460000 & -2.77813600 & -1.62701200 \\
\hline $\mathrm{H}$ & -9.49465400 & -4.67160900 & -0.98236300 \\
\hline $\mathrm{H}$ & -8.91962400 & -6.19777700 & -1.65827600 \\
\hline $\mathrm{H}$ & -10.43663700 & -5.89581600 & -3.72518600 \\
\hline $\mathrm{H}$ & -10.68240600 & -4.29968800 & -4.27963400 \\
\hline $\mathrm{H}$ & -8.89026000 & -3.45047600 & -3.02325800 \\
\hline $\mathrm{H}$ & 1.79443600 & -2.87581900 & -6.61511600 \\
\hline $\mathrm{H}$ & 0.67712600 & -3.35611200 & -7.93650700 \\
\hline $\mathrm{H}$ & -0.97834800 & -1.69126100 & -7.24929400 \\
\hline $\mathrm{H}$ & 0.56781000 & -0.83184400 & -7.20389800 \\
\hline $\mathrm{H}$ & -0.48865100 & -4.47632900 & -4.19538800 \\
\hline $\mathrm{H}$ & 1.23600300 & -4.04204600 & -4.32700000 \\
\hline $\mathrm{H}$ & -4.40778400 & 4.19055900 & -1.80580800 \\
\hline $\mathrm{H}$ & -4.36823600 & 3.79484800 & -3.54751800 \\
\hline $\mathrm{H}$ & -5.53895600 & 0.43967200 & -0.27363300 \\
\hline $\mathrm{H}$ & -5.90918900 & 0.13351300 & -1.97928100 \\
\hline $\mathrm{H}$ & -6.03596000 & 2.79733700 & -0.44618400 \\
\hline $\mathrm{H}$ & -7.33626800 & 2.00004000 & -1.37941000 \\
\hline $\mathrm{H}$ & 0.86217700 & 9.02153300 & -0.89832300 \\
\hline $\mathrm{H}$ & 5.06256800 & 7.49150900 & -4.45714900 \\
\hline
\end{tabular}




\begin{tabular}{|c|c|c|c|}
\hline $\mathrm{H}$ & 3.52733500 & 5.55138600 & -4.82208100 \\
\hline $\mathrm{H}$ & 1.93377000 & 4.83021700 & -3.05039200 \\
\hline $\mathrm{H}$ & 1.79389500 & 6.04714200 & -0.89495300 \\
\hline $\mathrm{H}$ & 5.72804300 & 9.44862200 & -2.46879700 \\
\hline $\mathrm{H}$ & 5.00008000 & 10.07286700 & -0.11394200 \\
\hline $\mathrm{H}$ & 2.41610600 & 7.37030900 & 1.21664000 \\
\hline $\mathrm{H}$ & 3.52262700 & 8.58968000 & 1.84245700 \\
\hline $\mathrm{H}$ & 1.05673000 & 8.57341400 & 2.96304200 \\
\hline $\mathrm{H}$ & 0.41109300 & 10.08485400 & 2.68005300 \\
\hline $\mathrm{H}$ & 2.10559600 & 10.39734100 & 0.87539400 \\
\hline $\mathrm{H}$ & 7.06531500 & -2.49269800 & 9.61494200 \\
\hline $\mathrm{H}$ & 4.30420000 & -3.86641100 & 8.32560900 \\
\hline $\mathrm{H}$ & 4.16980600 & -2.73470400 & 9.56272900 \\
\hline $\mathrm{H}$ & 6.08117500 & -2.74112300 & 7.29953900 \\
\hline $\mathrm{H}$ & 3.94897500 & -0.70957000 & 8.10121900 \\
\hline $\mathrm{H}$ & 5.41418400 & -0.15341700 & 7.32598200 \\
\hline $\mathrm{H}$ & 5.63190900 & -2.58084300 & 4.94406200 \\
\hline $\mathrm{H}$ & 4.05980100 & -2.36355500 & 2.96755900 \\
\hline $\mathrm{H}$ & 1.58488600 & -1.04915900 & 2.35546900 \\
\hline $\mathrm{H}$ & -0.18437400 & 0.34630100 & 3.44626100 \\
\hline $\mathrm{H}$ & -0.02360500 & 0.98637800 & 5.82556900 \\
\hline $\mathrm{H}$ & 1.95648400 & 0.32932500 & 7.19755600 \\
\hline $\mathrm{H}$ & 2.42444800 & -0.18111500 & -3.14461800 \\
\hline $\mathrm{H}$ & 2.02529400 & 0.83037800 & -4.54419200 \\
\hline $\mathrm{H}$ & 5.51398300 & 0.89549900 & -4.81152100 \\
\hline $\mathrm{H}$ & 4.18785500 & -0.16438800 & -4.96922100 \\
\hline $\mathrm{H}$ & 3.85575800 & 2.40891800 & -3.93756600 \\
\hline $\mathrm{H}$ & 5.07386400 & 3.31790500 & -2.36009200 \\
\hline $\mathrm{H}$ & 5.98666400 & 3.86613400 & -0.37839200 \\
\hline $\mathrm{H}$ & 4.46230200 & 1.55581800 & 0.92354900 \\
\hline $\mathrm{H}$ & 6.34088400 & 2.57136200 & 2.33601400 \\
\hline $\mathrm{H}$ & 5.59675900 & 4.16402500 & 2.12635200 \\
\hline $\mathrm{H}$ & 4.73542500 & 2.88426200 & 3.00575900 \\
\hline $\mathrm{H}$ & 2.61884700 & 3.64126200 & 2.63884400 \\
\hline $\mathrm{H}$ & 1.37520600 & 3.65398800 & 1.37904900 \\
\hline $\mathrm{H}$ & 2.14548000 & 2.12715000 & 1.85867900 \\
\hline $\mathrm{H}$ & 3.62962500 & 4.47627800 & 0.49323400 \\
\hline $\mathrm{H}$ & 2.96344100 & 3.09105300 & -0.37512900 \\
\hline $\mathrm{H}$ & 6.98408100 & 1.00160600 & -0.71010700 \\
\hline $\mathrm{H}$ & -4.76233700 & -0.25368400 & 4.82648900 \\
\hline $\mathrm{H}$ & -5.33879600 & -3.19193600 & 2.88225200 \\
\hline $\mathrm{H}$ & -5.10344000 & -1.64324700 & 2.24251300 \\
\hline $\mathrm{H}$ & -3.81829200 & -2.92509400 & 4.58829200 \\
\hline $\mathrm{H}$ & -1.65854400 & -2.12552300 & 3.77480700 \\
\hline $\mathrm{H}$ & -2.44370300 & -3.07178000 & 2.50832300 \\
\hline $\mathrm{H}$ & -11.48433800 & 4.56643600 & 3.81619400 \\
\hline $\mathrm{H}$ & -9.89062000 & 2.68293800 & 1.45634700 \\
\hline $\mathrm{H}$ & -10.38742500 & 4.25195700 & 1.12651200 \\
\hline $\mathrm{H}$ & -9.26558300 & 3.16105300 & 3.64560500 \\
\hline $\mathrm{H}$ & -8.33283900 & 5.48191100 & 1.88805600 \\
\hline
\end{tabular}




\begin{tabular}{|c|c|c|c|}
\hline $\mathrm{H}$ & -7.95169800 & 5.44183700 & 3.59753500 \\
\hline $\mathrm{H}$ & -7.31871800 & 1.83458300 & 3.02065500 \\
\hline $\mathrm{H}$ & -4.86768700 & 1.42160400 & 2.36304900 \\
\hline $\mathrm{H}$ & -2.69932300 & 2.87487100 & 1.33409900 \\
\hline $\mathrm{H}$ & -2.05386100 & 5.20495800 & 0.66356700 \\
\hline $\mathrm{H}$ & -3.68019800 & 7.07046600 & 0.81859400 \\
\hline $\mathrm{H}$ & -6.01023900 & 6.67516800 & 1.62016600 \\
\hline $\mathrm{H}$ & 7.34881800 & -2.70752300 & -4.65294200 \\
\hline $\mathrm{H}$ & 2.12973300 & -5.93515400 & -4.98208400 \\
\hline $\mathrm{H}$ & 2.45595700 & -5.12603200 & -6.52097400 \\
\hline $\mathrm{H}$ & 2.79177000 & -6.86386900 & -6.34124700 \\
\hline $\mathrm{H}$ & 4.07371100 & -6.63393100 & -3.52294900 \\
\hline $\mathrm{H}$ & 5.04211500 & -7.43561200 & -4.66198600 \\
\hline $\mathrm{H}$ & 4.93325600 & -5.70469800 & -6.21022900 \\
\hline $\mathrm{H}$ & 5.71129000 & -4.90009600 & -3.47692200 \\
\hline $\mathrm{H}$ & 5.02495200 & -1.96470400 & -3.71465800 \\
\hline $\mathrm{H}$ & 5.49484100 & -1.46449400 & -1.48157700 \\
\hline $\mathrm{H}$ & 4.35078900 & -2.78959100 & -1.48685400 \\
\hline $\mathrm{H}$ & 6.17638100 & -4.41191900 & -0.85715300 \\
\hline $\mathrm{H}$ & 7.36688800 & -3.09154200 & -0.91838300 \\
\hline $\mathrm{H}$ & 6.52806800 & -1.03050500 & 2.29531100 \\
\hline $\mathrm{H}$ & 7.58111700 & -1.17650100 & 0.86147800 \\
\hline $\mathrm{H}$ & 5.88557200 & -0.61121800 & 0.68502400 \\
\hline $\mathrm{H}$ & -0.81505700 & -7.32659300 & 3.69754900 \\
\hline $\mathrm{H}$ & 2.13264100 & -8.06355400 & 4.36390700 \\
\hline $\mathrm{H}$ & 2.45142700 & -6.45803200 & 3.87015300 \\
\hline $\mathrm{H}$ & 0.94659100 & -6.93326500 & 6.18613400 \\
\hline $\mathrm{H}$ & 1.76574800 & -4.74967900 & 5.22946500 \\
\hline $\mathrm{H}$ & -0.85277200 & -4.68794000 & 3.60617500 \\
\hline $\mathrm{H}$ & 0.42746300 & -3.46359800 & 3.64623000 \\
\hline $\mathrm{H}$ & 0.72271900 & -5.04034100 & 2.88835700 \\
\hline $\mathrm{H}$ & 0.64184600 & -5.63759200 & 7.95530500 \\
\hline $\mathrm{H}$ & 1.55319300 & -4.13623900 & 7.65668100 \\
\hline $\mathrm{H}$ & -0.05108900 & -4.07429600 & 8.41305400 \\
\hline $\mathrm{H}$ & -0.22678200 & -3.37141500 & 6.04296100 \\
\hline $\mathrm{H}$ & -1.10998200 & -4.88063000 & 6.27828600 \\
\hline $\mathrm{H}$ & -1.24022600 & -3.60818600 & -0.51577200 \\
\hline $\mathrm{H}$ & -0.90700100 & -2.72864200 & 0.79346800 \\
\hline $\mathrm{H}$ & 0.85340900 & -3.74013700 & -0.13891600 \\
\hline $\mathrm{H}$ & 1.21654200 & -3.20558300 & -1.58150300 \\
\hline $\mathrm{H}$ & 2.22576800 & -1.98515700 & -0.32798300 \\
\hline $\mathrm{H}$ & 3.03331300 & -0.53006400 & -0.63530500 \\
\hline $\mathrm{H}$ & 2.58667700 & -4.92673400 & -1.39233300 \\
\hline $\mathrm{H}$ & 3.86615200 & -4.76763100 & -2.33665200 \\
\hline $\mathrm{H}$ & 1.06584600 & -0.34263600 & -0.28028800 \\
\hline $\mathrm{H}$ & 0.37054900 & 1.16687200 & -0.12935900 \\
\hline $\mathrm{H}$ & -0.89477900 & -0.41622600 & 0.67856400 \\
\hline
\end{tabular}

XE-ES,$E=-5430.221643$ 


\begin{tabular}{|c|c|c|c|}
\hline $\mathrm{C}$ & -4.11343300 & -1.15990700 & 3.89251600 \\
\hline $\mathrm{C}$ & -3.72068200 & 0.04082500 & 4.78734400 \\
\hline $\mathrm{O}$ & -2.79509200 & -0.00132700 & 5.61403000 \\
\hline$C$ & -2.88909200 & -1.52172600 & 3.03185400 \\
\hline $\mathrm{C}$ & -2.62731000 & -0.39920200 & 2.05107900 \\
\hline $\mathrm{O}$ & -3.15498200 & 0.73404300 & 2.12824400 \\
\hline $\mathrm{O}$ & -1.77129700 & -0.74435400 & 1.05255300 \\
\hline $\mathrm{N}$ & 0.14946600 & -7.41181400 & 3.86630700 \\
\hline $\mathrm{C}$ & -0.75441300 & -7.02876100 & 4.96724600 \\
\hline $\mathrm{C}$ & -2.20700700 & -6.91504200 & 4.48597400 \\
\hline $\mathrm{O}$ & -3.18389900 & -7.43371400 & 5.06348400 \\
\hline $\mathrm{C}$ & -0.26916000 & -5.69936000 & 5.63117600 \\
\hline $\mathrm{C}$ & -0.85785900 & -5.50787700 & 7.05403900 \\
\hline $\mathrm{C}$ & -0.55651000 & -4.44909000 & 4.76072400 \\
\hline $\mathrm{C}$ & -0.30975900 & -6.48703700 & 8.11473200 \\
\hline $\mathrm{N}$ & 2.51338500 & -7.01781100 & -4.52028700 \\
\hline $\mathrm{C}$ & 2.79086700 & -6.15857800 & -5.69554900 \\
\hline $\mathrm{C}$ & 3.37467700 & -4.80435900 & -5.21441400 \\
\hline $\mathrm{O}$ & 3.33380300 & -3.74948000 & -5.91670200 \\
\hline $\mathrm{C}$ & 1.62255400 & -5.97547700 & -6.68621100 \\
\hline $\mathrm{N}$ & 3.98581200 & -4.86226400 & -3.98971000 \\
\hline $\mathrm{C}$ & 4.61635900 & -3.69107500 & -3.36182100 \\
\hline $\mathrm{C}$ & 6.03965900 & -3.52250400 & -3.89814700 \\
\hline $\mathrm{O}$ & 6.98308500 & -2.98807400 & -3.28671800 \\
\hline $\mathrm{C}$ & 4.50394500 & -3.64968300 & -1.81788300 \\
\hline $\mathrm{C}$ & 5.40792800 & -4.63546200 & -1.05272200 \\
\hline $\mathrm{S}$ & 5.29630100 & -4.52373200 & 0.79701500 \\
\hline $\mathrm{C}$ & 6.19317100 & -2.93299000 & 1.07717600 \\
\hline $\mathrm{N}$ & 4.69999900 & -0.41404700 & -4.50866700 \\
\hline $\mathrm{C}$ & 4.14223900 & 0.62926300 & -3.64289300 \\
\hline $\mathrm{C}$ & 4.74976900 & 0.47874800 & -2.20389600 \\
\hline $\mathrm{O}$ & 4.41503800 & -0.46028300 & -1.42655400 \\
\hline $\mathrm{C}$ & 2.61819000 & 0.46063200 & -3.56759300 \\
\hline $\mathrm{S}$ & 1.77862200 & 1.79405200 & -2.56880100 \\
\hline $\mathrm{N}$ & 5.68605600 & 1.41341100 & -1.83328700 \\
\hline $\mathrm{C}$ & 6.36530900 & 1.49155900 & -0.50867400 \\
\hline $\mathrm{C}$ & 7.35798700 & 0.32738100 & -0.34801400 \\
\hline $\mathrm{O}$ & 8.43112700 & 0.42702300 & 0.27413400 \\
\hline $\mathrm{C}$ & 5.39125700 & 1.59110000 & 0.72746300 \\
\hline $\mathrm{C}$ & 4.33033500 & 2.69638700 & 0.48636200 \\
\hline $\mathrm{C}$ & 6.19942000 & 1.85713100 & 2.01929700 \\
\hline $\mathrm{C}$ & 3.27208200 & 2.79282500 & 1.60643200 \\
\hline $\mathrm{N}$ & -8.94521500 & 5.95431900 & 2.15192700 \\
\hline $\mathrm{C}$ & -8.05341200 & 6.20957200 & 3.31210500 \\
\hline $\mathrm{C}$ & -8.75733900 & 7.22333000 & 4.23444300 \\
\hline $\mathrm{O}$ & -8.18664500 & 8.03931600 & 4.97859000 \\
\hline $\mathrm{C}$ & -6.59428900 & 6.64400800 & 3.01312900 \\
\hline $\mathrm{C}$ & -5.59834900 & 5.56423500 & 2.64541500 \\
\hline $\mathrm{C}$ & -5.67411700 & 4.18000000 & 2.75396800 \\
\hline $\mathrm{C}$ & -4.26003900 & 5.85963600 & 2.16037600 \\
\hline
\end{tabular}




\begin{tabular}{|c|c|c|c|}
\hline $\mathrm{N}$ & -4.45811200 & 3.60009100 & 2.35987200 \\
\hline $\mathrm{C}$ & -3.57667500 & 4.61528300 & 1.99075600 \\
\hline $\mathrm{C}$ & -3.57991300 & 7.06614700 & 1.86040900 \\
\hline $\mathrm{C}$ & -2.25165200 & 4.54045200 & 1.51825900 \\
\hline $\mathrm{C}$ & -2.25887400 & 7.00118800 & 1.39761600 \\
\hline $\mathrm{C}$ & -1.60455500 & 5.74836300 & 1.22420200 \\
\hline $\mathrm{N}$ & 3.65027700 & 8.83773200 & 2.80883700 \\
\hline $\mathrm{C}$ & 4.04936700 & 8.77205400 & 1.39801900 \\
\hline $\mathrm{C}$ & 2.87571200 & 8.82878800 & 0.40878400 \\
\hline $\mathrm{O}$ & 1.69351300 & 9.01510500 & 0.75633700 \\
\hline $\mathrm{C}$ & 4.94073600 & 7.51144300 & 1.15633600 \\
\hline $\mathrm{C}$ & 5.61163900 & 7.44584900 & -0.19499900 \\
\hline $\mathrm{C}$ & 6.78653800 & 8.08705400 & -0.55816100 \\
\hline $\mathrm{C}$ & 5.17436100 & 6.69552400 & -1.36221100 \\
\hline $\mathrm{N}$ & 7.10406300 & 7.78323400 & -1.88685300 \\
\hline $\mathrm{C}$ & 6.13037200 & 6.92499100 & -2.40354600 \\
\hline $\mathrm{C}$ & 4.07425000 & 5.84087000 & -1.61897700 \\
\hline $\mathrm{C}$ & 6.01445500 & 6.33354500 & -3.67630000 \\
\hline $\mathrm{C}$ & 3.95152200 & 5.24425500 & -2.88135000 \\
\hline $\mathrm{C}$ & 4.91355200 & 5.49134600 & -3.90054200 \\
\hline $\mathrm{N}$ & 4.09831200 & -4.47479000 & 8.58322900 \\
\hline $\mathrm{C}$ & 4.98897700 & -3.54199600 & 7.87318700 \\
\hline $\mathrm{C}$ & 6.22071100 & -3.49067100 & 8.76006600 \\
\hline $\mathrm{O}$ & 6.80990100 & -2.46000900 & 9.13535500 \\
\hline $\mathrm{C}$ & 4.43459600 & -2.13876200 & 7.53143600 \\
\hline $\mathrm{C}$ & 3.62472300 & -2.04684200 & 6.25843400 \\
\hline $\mathrm{C}$ & 3.81643100 & -2.71936900 & 5.05766500 \\
\hline $\mathrm{C}$ & 2.55076400 & -1.09821500 & 6.02685300 \\
\hline $\mathrm{N}$ & 2.92136400 & -2.23987400 & 4.09424200 \\
\hline $\mathrm{C}$ & 2.13463700 & -1.24049500 & 4.66421700 \\
\hline $\mathrm{C}$ & 1.90696000 & -0.13950900 & 6.84765000 \\
\hline $\mathrm{C}$ & 1.10652500 & -0.45262500 & 4.10739100 \\
\hline $\mathrm{C}$ & 0.87713900 & 0.63709500 & 6.30247200 \\
\hline $\mathrm{C}$ & 0.48297500 & 0.48278800 & 4.94436600 \\
\hline $\mathrm{N}$ & -11.10892400 & -2.01359100 & -3.56014800 \\
\hline $\mathrm{C}$ & -9.79416500 & -2.31393400 & -2.95586000 \\
\hline $\mathrm{C}$ & -8.91770200 & -2.72399300 & -4.13644400 \\
\hline $\mathrm{O}$ & -8.75757600 & -3.90199700 & -4.51349400 \\
\hline $\mathrm{C}$ & -9.89086700 & -3.39675800 & -1.85635700 \\
\hline $\mathrm{C}$ & -8.55147000 & -3.67555800 & -1.18339600 \\
\hline $\mathrm{C}$ & -8.06421400 & -2.82378900 & -0.16422300 \\
\hline $\mathrm{C}$ & -7.75621900 & -4.77349600 & -1.57999700 \\
\hline $\mathrm{C}$ & -6.81585900 & -3.05646600 & 0.44369500 \\
\hline $\mathrm{C}$ & -6.50953900 & -5.01755900 & -0.97169900 \\
\hline $\mathrm{C}$ & -6.03413500 & -4.15896800 & 0.04052100 \\
\hline $\mathrm{C}$ & -0.62319400 & -3.46511700 & -4.85929100 \\
\hline $\mathrm{O}$ & -0.86186500 & -3.56797300 & -6.28172200 \\
\hline $\mathrm{C}$ & -0.56945200 & -2.01844200 & -4.44640500 \\
\hline $\mathrm{S}$ & -0.78014200 & -1.64971200 & -2.72335300 \\
\hline $\mathrm{C}$ & -0.45389400 & -1.00598200 & -5.34697200 \\
\hline
\end{tabular}




\begin{tabular}{|c|c|c|c|}
\hline $\mathrm{S}$ & -0.45724300 & 0.68524700 & -4.80585800 \\
\hline $\mathrm{C}$ & -0.31682800 & -1.25313000 & -6.84006800 \\
\hline $\mathrm{C}$ & 0.03412800 & -2.72772900 & -7.08027200 \\
\hline $\mathrm{C}$ & -3.50714800 & 4.57298900 & -2.36323600 \\
\hline $\mathrm{O}$ & -4.97320000 & 4.51097800 & -2.35381200 \\
\hline $\mathrm{C}$ & -2.86948100 & 3.20606800 & -2.15754400 \\
\hline $\mathrm{S}$ & -1.23258300 & 2.97068200 & -2.80411100 \\
\hline $\mathrm{C}$ & -3.56092000 & 2.16912400 & -1.61372700 \\
\hline S & -2.79352100 & 0.55429100 & -1.49120100 \\
\hline $\mathrm{C}$ & -5.02418600 & 2.29498800 & -1.21612700 \\
\hline $\mathrm{C}$ & -5.46477500 & 3.77639300 & -1.18648700 \\
\hline $\mathrm{W}$ & -0.58333800 & 0.69051100 & -2.47011600 \\
\hline $\mathrm{O}$ & -3.43977200 & -3.57526900 & -2.07947300 \\
\hline $\mathrm{O}$ & 0.51054100 & -6.53076400 & -2.42617400 \\
\hline $\mathrm{O}$ & -1.23449600 & -3.68969500 & 1.14259900 \\
\hline $\mathrm{O}$ & -1.15698500 & -4.64533100 & -1.46115000 \\
\hline $\mathrm{O}$ & 0.48380000 & 0.23788400 & -0.60917300 \\
\hline $\mathrm{H}$ & -8.50962400 & -1.88176000 & -4.73255900 \\
\hline $\mathrm{H}$ & -8.10764500 & -5.42355100 & -2.37691700 \\
\hline $\mathrm{H}$ & -5.91842300 & -5.87669900 & -1.28178500 \\
\hline $\mathrm{H}$ & -5.08127500 & -4.35424900 & 0.52704100 \\
\hline $\mathrm{H}$ & -6.44218100 & -2.39857000 & 1.22548200 \\
\hline $\mathrm{H}$ & -8.67048200 & -1.97817300 & 0.16032800 \\
\hline $\mathrm{H}$ & -10.63147900 & -3.04511400 & -1.12755200 \\
\hline $\mathrm{H}$ & -10.28037900 & -4.31719400 & -2.30945500 \\
\hline $\mathrm{H}$ & -11.54288200 & -2.77279900 & -4.07556900 \\
\hline $\mathrm{H}$ & -11.23862400 & -1.09441000 & -3.96731400 \\
\hline $\mathrm{H}$ & -9.40529700 & -1.38430700 & -2.52312400 \\
\hline $\mathrm{H}$ & 1.08086100 & -2.93188900 & -6.81053800 \\
\hline $\mathrm{H}$ & -0.14106400 & -3.02577500 & -8.11880200 \\
\hline $\mathrm{H}$ & -1.26106100 & -0.99947500 & -7.34327000 \\
\hline $\mathrm{H}$ & 0.46117500 & -0.60257700 & -7.26503000 \\
\hline $\mathrm{H}$ & -1.45862400 & -3.98993000 & -4.38280800 \\
\hline $\mathrm{H}$ & 0.31195000 & -3.98533800 & -4.58577600 \\
\hline $\mathrm{H}$ & -3.16788100 & 5.27600200 & -1.58406200 \\
\hline $\mathrm{H}$ & -3.23933700 & 4.97857400 & -3.34364400 \\
\hline $\mathrm{H}$ & -5.19544100 & 1.85044300 & -0.22448600 \\
\hline $\mathrm{H}$ & -5.64080300 & 1.73160700 & -1.93334100 \\
\hline $\mathrm{H}$ & -5.10083300 & 4.26298600 & -0.27068100 \\
\hline $\mathrm{H}$ & -6.55594100 & 3.86336500 & -1.22984900 \\
\hline $\mathrm{H}$ & 3.14019400 & 8.72397900 & -0.66009800 \\
\hline $\mathrm{H}$ & 6.74483200 & 6.52055400 & -4.46064400 \\
\hline $\mathrm{H}$ & 4.78450100 & 5.02490100 & -4.87470000 \\
\hline $\mathrm{H}$ & 3.11513200 & 4.57954500 & -3.08285000 \\
\hline $\mathrm{H}$ & 3.33003200 & 5.63940000 & -0.85202600 \\
\hline $\mathrm{H}$ & 7.91594000 & 8.11740800 & -2.38525300 \\
\hline $\mathrm{H}$ & 7.42543800 & 8.72701100 & 0.03428400 \\
\hline $\mathrm{H}$ & 4.32783300 & 6.61204500 & 1.31508700 \\
\hline$\Pi$ & 5.69062400 & 7.52524400 & 1.95669800 \\
\hline$\pi$ & 3.29256600 & 7.94938300 & 3.15998700 \\
\hline
\end{tabular}




$\begin{array}{lrrr}\mathrm{H} & 2.97588800 & 9.57920900 & 2.99583100 \\ \mathrm{H} & 4.67466600 & 9.65042800 & 1.16473700 \\ \mathrm{H} & 6.54968600 & -4.49409600 & 9.09331700 \\ \mathrm{H} & 3.43284100 & -4.96569500 & 7.99207900 \\ \mathrm{H} & 3.64842500 & -4.07316900 & 9.40315500 \\ \mathrm{H} & 5.31064700 & -4.05238500 & 6.95204600 \\ \mathrm{H} & 3.82723800 & -1.79383900 & 8.38093600 \\ \mathrm{H} & 5.28588500 & -1.44380500 & 7.48341600 \\ \mathrm{H} & 4.52614700 & -3.49320900 & 4.80039300 \\ \mathrm{H} & 2.86976800 & -2.53947800 & 3.12705500 \\ \mathrm{H} & 0.80966400 & -0.56371800 & 3.06802800 \\ \mathrm{H} & -0.32304100 & 1.10051900 & 4.55766800 \\ \mathrm{H} & 0.35941800 & 1.36683300 & 6.91969800 \\ \mathrm{H} & 2.20491900 & -0.00556500 & 7.88557900 \\ \mathrm{H} & 2.39028600 & -0.52460000 & -3.14761000 \\ \mathrm{H} & 2.20286200 & 0.50960400 & -4.57944400 \\ \mathrm{H} & 5.66136500 & -0.31458400 & -4.81156800 \\ \mathrm{H} & 4.07392900 & -0.83806200 & -5.18487600 \\ \mathrm{H} & 4.36761300 & 1.65049500 & -4.00457900 \\ \mathrm{H} & 5.84652500 & 2.18698700 & -2.46983500 \\ \mathrm{H} & 6.95375600 & 2.41973200 & -0.51916400 \\ \mathrm{H} & 4.86881500 & 0.63044800 & 0.82390000 \\ \mathrm{H} & 6.98243000 & 1.11115200 & 2.18681400 \\ \mathrm{H} & -0.58472500 & 5.73117800 & 0.84810600 \\ \mathrm{H} & -1.71701700 & 7.91395900 & 1.16162600 \\ \mathrm{H} & -4.07044600 & 8.02895000 & 1.98738600 \\ \mathrm{H} & 6.17660000 & -3.89958800 & -4.93183100 \\ \mathrm{H} & & & \\ \mathrm{H} & 5.53359900 & 1.84163200 & 2.88890300 \\ \mathrm{H} & 3.69655300 & 3.14299100 & 2.55521200 \\ \mathrm{H} & 2.47264400 & 3.48876900 & 1.31961300 \\ \mathrm{H} & 2.80426900 & 1.81662300 & 1.79507200 \\ \mathrm{H} & 4.84495300 & 3.66516200 & 0.37819400 \\ \mathrm{H} & 3.81068900 & 2.50782600 & -0.45989000 \\ \mathrm{H} & 7.06161600 & -0.61900400 & -0.83229500 \\ \mathrm{H} & -4.34460400 & 0.94916400 & 4.66007100 \\ \mathrm{H} & -6.19409800 & -1.04665400 & 3.53256500 \\ \mathrm{H} & -5.25122400 & -0.14893900 & 2.43607800 \\ \mathrm{H} & -4.30490900 & -2.00884700 & 4.56261900 \\ \mathrm{H} & -2.00225600 & -1.65835000 & 3.66427600 \\ \mathrm{H} & -3.05272000 & -2.45717300 & 2.48987300 \\ \mathrm{H} & -9.86257700 & 7.15561700 & 4.18404200 \\ \mathrm{H} & -8.77986900 & 5.05852100 & 1.69957300 \\ \mathrm{H} & -6.93617100 & 6.71211100 & 1.47035000 \\ \mathrm{H} & -1.7536700 & 5.27684200 & 3.90219400 \\ \mathrm{H} & 7.39543300 & 2.20746700 \\ \mathrm{H} & & & \end{array}$




$\begin{array}{lrrr}\mathrm{H} & 0.73712500 & -5.54966000 & -6.20511600 \\ \mathrm{H} & 1.91326700 & -5.30843300 & -7.50547200 \\ \mathrm{H} & 1.34571400 & -6.95164700 & -7.10636600 \\ \mathrm{H} & 1.69285900 & -6.74625200 & -3.95667300 \\ \mathrm{H} & 2.48933500 & -8.00832000 & -4.75218000 \\ \mathrm{H} & 3.62013900 & -6.63491500 & -6.24493700 \\ \mathrm{H} & 3.88457400 & -5.76004600 & -3.51301700 \\ \mathrm{H} & 4.09648100 & -2.79806400 & -3.74401700 \\ \mathrm{H} & 4.75085800 & -2.62755800 & -1.52578400 \\ \mathrm{H} & 3.45492100 & -3.81458500 & -1.54521600 \\ \mathrm{H} & 5.14400800 & -5.68063700 & -1.27395500 \\ \mathrm{H} & 6.45874800 & -4.49325500 & -1.32673800 \\ \mathrm{H} & 6.24815100 & -2.78070900 & 2.16120100 \\ \mathrm{H} & 7.20957700 & -2.98752300 & 0.67200700 \\ \mathrm{H} & 5.65807500 & -2.09293100 & 0.62441700 \\ \mathrm{H} & -2.33925000 & -6.36101000 & 3.53353800 \\ \mathrm{H} & -0.03142100 & -8.31424300 & 3.43696500 \\ \mathrm{H} & 0.36263900 & -6.67973800 & 3.19432800 \\ \mathrm{H} & -0.72588000 & -7.82312500 & 5.72248700 \\ \mathrm{H} & 0.82192000 & -5.81775400 & 5.72019400 \\ \mathrm{H} & -1.62155600 & -4.18026000 & 4.79724800 \\ \mathrm{H} & 0.01215200 & -3.59133800 & 5.13929100 \\ \mathrm{H} & -0.29322400 & -4.57914200 & 3.70333600 \\ \mathrm{H} & -0.56935100 & -7.52864700 & 7.88681400 \\ \mathrm{H} & 0.78597800 & -6.42257700 & 8.18089600 \\ \mathrm{H} & -0.72090700 & -6.25677900 & 9.10671300 \\ \mathrm{H} & -0.63561300 & -4.47917800 & 7.37314200 \\ \mathrm{H} & -1.95460600 & -5.58821200 & 7.01219700 \\ \mathrm{H} & -4.38877400 & -3.60883600 & -1.85003500 \\ \mathrm{H} & -3.14271000 & -2.68019100 & -2.36001400 \\ \mathrm{H} & -2.12244100 & -4.46374900 & -1.70135400 \\ \mathrm{H} & -0.65776900 & -3.84519200 & -1.75795800 \\ \mathrm{H} & -1.22783600 & -4.20700600 & 0.29227900 \\ \mathrm{H} & -1.48285200 & -2.75527500 & 0.96405000 \\ \mathrm{H} & -0.21132200 & -5.91058100 & -2.11576800 \\ \mathrm{H} & 1.00838900 & -6.86805000 & -1.65847700 \\ \mathrm{H} & 0.57581900 & -0.61856500 & -0.13690000 \\ \mathrm{H} & 1.35556300 & 0.72897200 & -0.71787400 \\ \mathrm{H} & -1.80485200 & -0.07952900 & 0.28472400 \\ & 2.44156600 & -1.93833300 & 0.43853700 \\ \mathrm{H} & 1.51248200 & -2.55281500 & 0.95047700 \\ & 0.66142700 & -3.10856500 & 1.32085500\end{array}$

XE-TS , $E=-5430.179457$

$\begin{array}{lrrc}\mathrm{N} & -5.43699100 & -0.74410400 & 2.74273000 \\ \mathrm{C} & -4.44090700 & -1.11162200 & 3.75892000 \\ \mathrm{C} & -3.86064100 & 0.06065800 & 4.58800800 \\ \mathrm{O} & -3.29357800 & -0.12144900 & 5.67868100 \\ \mathrm{C} & -3.26551700 & -1.88604700 & 3.13097600 \\ & & & \mathrm{~S} 45\end{array}$




\begin{tabular}{|c|c|c|c|}
\hline $\mathrm{C}$ & -2.32049100 & -0.98379900 & 2.34868400 \\
\hline $\mathrm{O}$ & -2.45277600 & 0.24188500 & 2.20891200 \\
\hline $\mathrm{O}$ & -1.28070600 & -1.71441900 & 1.84853900 \\
\hline $\mathrm{N}$ & -0.41793100 & -7.56030600 & 3.3892990 \\
\hline $\mathrm{C}$ & -1.35863300 & -7.27890700 & 4.48708700 \\
\hline $\mathrm{C}$ & -2.76152500 & -6.95053500 & 3.95452800 \\
\hline $\mathrm{O}$ & -3.82047600 & -7.44605700 & 4.3866780 \\
\hline $\mathrm{C}$ & -0.80873500 & -6.14067500 & 5.40527200 \\
\hline $\mathrm{C}$ & -1.60306700 & -6.00594900 & 6.7312950 \\
\hline $\mathrm{C}$ & -0.73748700 & -4.77261300 & 4.6836430 \\
\hline $\mathrm{C}$ & -1.53386100 & -7.23853400 & 7.6590450 \\
\hline $\mathrm{N}$ & 2.20240700 & -6.76826100 & -4.8281770 \\
\hline $\mathrm{C}$ & 2.65177100 & -5.96930100 & -5.9927230 \\
\hline $\mathrm{C}$ & 3.37589700 & -4.69910000 & -5.4748400 \\
\hline $\mathrm{O}$ & 3.46599500 & -3.63517800 & -6.1539350 \\
\hline $\mathrm{C}$ & 1.57651800 & -5.64586500 & -7.0502850 \\
\hline $\mathrm{N}$ & 3.94028200 & -4.85356900 & -4.2352850 \\
\hline $\mathrm{C}$ & 4.64417500 & -3.77505700 & -3.5227660 \\
\hline $\mathrm{C}$ & 6.11124400 & -3.74445800 & -3.95072000 \\
\hline $\mathrm{O}$ & 7.06425900 & -3.36914000 & -3.2437710 \\
\hline $\mathrm{C}$ & 4.40720000 & -3.79592100 & -1.9924600 \\
\hline $\mathrm{C}$ & 5.18025700 & -4.86156800 & -1.1860170 \\
\hline S & 4.74980300 & -4.85062500 & 0.62243400 \\
\hline $\mathrm{C}$ & 5.52119300 & -3.24547100 & 1.12648500 \\
\hline $\mathrm{N}$ & 4.86611700 & -0.40371700 & -4.45715200 \\
\hline $\mathrm{C}$ & 4.33445700 & 0.60209200 & -3.52296200 \\
\hline $\mathrm{C}$ & 4.90608400 & 0.30231800 & -2.09816200 \\
\hline $\mathrm{O}$ & 4.56531000 & -0.71923700 & -1.4409390 \\
\hline $\mathrm{C}$ & 2.80179800 & 0.50232100 & -3.49476400 \\
\hline S & 2.00079700 & 1.87763500 & -2.52627400 \\
\hline $\mathrm{N}$ & 5.83354600 & 1.19389800 & -1.60417000 \\
\hline $\mathrm{C}$ & 6.44646700 & 1.12794300 & -0.24778300 \\
\hline $\mathrm{C}$ & 7.39998600 & -0.07497400 & -0.1417630 \\
\hline $\mathrm{O}$ & 8.48325700 & -0.03009500 & 0.47071700 \\
\hline $\mathrm{C}$ & 5.39954100 & 1.14021900 & 0.92973900 \\
\hline $\mathrm{C}$ & 4.43754900 & 2.34670100 & $0.7685550 \mathrm{C}$ \\
\hline $\mathrm{C}$ & 6.12629800 & 1.17269400 & 2.29434700 \\
\hline $\mathrm{C}$ & 3.28630800 & 2.36387900 & 1.79641400 \\
\hline $\mathrm{N}$ & -8.61413800 & 6.42118900 & 2.10639700 \\
\hline $\mathrm{C}$ & -7.80492600 & 6.49752300 & 3.34780300 \\
\hline $\mathrm{C}$ & -8.42793800 & 7.56364700 & 4.26108700 \\
\hline $\mathrm{O}$ & -7.81027100 & 8.24489000 & 5.09788800 \\
\hline $\mathrm{C}$ & -6.27692100 & 6.70710000 & $3.1897120 \mathrm{C}$ \\
\hline $\mathrm{C}$ & -5.47478300 & 5.48343700 & 2.81049800 \\
\hline $\mathrm{C}$ & -5.85202400 & 4.14785100 & 2.80639700 \\
\hline $\mathrm{C}$ & -4.07058300 & 5.51429400 & 2.43176400 \\
\hline $\mathrm{N}$ & -4.75701800 & 3.34706900 & 2.4464190 \\
\hline $\mathrm{C}$ & -3.65056800 & 4.16638400 & 2.20923000 \\
\hline $\mathrm{C}$ & -3.13402100 & 6.56259000 & 2.25542300 \\
\hline $\mathrm{C}$ & -2.34352600 & 3.83028900 & 1.8068350 \\
\hline
\end{tabular}




\begin{tabular}{|c|c|c|c|}
\hline C & -1.82956600 & 6.24085900 & 1.85656800 \\
\hline $\mathrm{C}$ & -1.44232000 & 4.88927000 & 1.63067400 \\
\hline $\mathrm{N}$ & 4.09865000 & 8.49198000 & 3.35182400 \\
\hline $\mathrm{C}$ & 4.54658500 & 8.45573200 & 1.95424900 \\
\hline $\mathrm{C}$ & 3.41650500 & 8.63825900 & 0.93010500 \\
\hline $\mathrm{O}$ & 2.24642700 & 8.92788400 & 1.24717300 \\
\hline $\mathrm{C}$ & 5.35926600 & 7.14879600 & 1.68691300 \\
\hline $\mathrm{C}$ & 6.07555400 & 7.09920200 & 0.35827900 \\
\hline $\mathrm{C}$ & 7.29201200 & 7.69501200 & 0.06032800 \\
\hline $\mathrm{C}$ & 5.64456400 & 6.41443000 & -0.85087700 \\
\hline $\mathrm{N}$ & 7.64165500 & 7.42497000 & -1.26756900 \\
\hline $\mathrm{C}$ & 6.64690800 & 6.63489200 & -1.84951200 \\
\hline $\mathrm{C}$ & 4.51524600 & 5.62373000 & -1.17702500 \\
\hline $\mathrm{C}$ & 6.54861300 & 6.09683000 & -3.14717100 \\
\hline $\mathrm{C}$ & 4.40877400 & 5.08061500 & -2.46491300 \\
\hline $\mathrm{C}$ & 5.41757500 & 5.31816600 & -3.44043200 \\
\hline $\mathrm{N}$ & 3.52194500 & -5.13076000 & 8.39365000 \\
\hline $\mathrm{C}$ & 4.47988200 & -4.19195100 & 7.78475300 \\
\hline $\mathrm{C}$ & 5.69200100 & -4.29224500 & 8.69709700 \\
\hline $\mathrm{O}$ & 6.31664300 & -3.33491500 & 9.18968600 \\
\hline $\mathrm{C}$ & 4.01502200 & -2.73523200 & 7.56545200 \\
\hline $\mathrm{C}$ & 3.20973500 & -2.47545600 & 6.31297800 \\
\hline $\mathrm{C}$ & 3.16636600 & -3.19319200 & 5.12476200 \\
\hline $\mathrm{C}$ & 2.40382000 & -1.28582700 & 6.10092900 \\
\hline $\mathrm{N}$ & 2.37799800 & -2.51233000 & 4.18559500 \\
\hline $\mathrm{C}$ & 1.90253100 & -1.33433600 & 4.76184900 \\
\hline $\mathrm{C}$ & 2.07290200 & -0.18021900 & 6.92182200 \\
\hline $\mathrm{C}$ & 1.09600200 & -0.31168300 & 4.22574800 \\
\hline $\mathrm{C}$ & 1.26020500 & 0.83341200 & 6.39739300 \\
\hline $\mathrm{C}$ & 0.77825100 & 0.76863900 & 5.06045800 \\
\hline $\mathrm{N}$ & -11.04129000 & -1.08124300 & -4.10805900 \\
\hline $\mathrm{C}$ & -9.78562500 & -1.52852400 & -3.47467600 \\
\hline $\mathrm{C}$ & -8.87711700 & -1.89587600 & -4.64747200 \\
\hline $\mathrm{O}$ & -8.72723000 & -3.05502400 & -5.08229800 \\
\hline $\mathrm{C}$ & -10.03773000 & -2.69056500 & -2.48684400 \\
\hline $\mathrm{C}$ & -8.80462600 & -3.09456000 & -1.68992200 \\
\hline $\mathrm{C}$ & -8.45262700 & -2.40110900 & -0.50908500 \\
\hline $\mathrm{C}$ & -7.98613800 & -4.16650700 & -2.11346300 \\
\hline $\mathrm{C}$ & -7.31648900 & -2.76940100 & 0.23844600 \\
\hline $\mathrm{C}$ & -6.85480200 & -4.54545400 & -1.36838000 \\
\hline $\mathrm{C}$ & -6.51804300 & -3.85134600 & -0.18872200 \\
\hline $\mathrm{C}$ & -0.54579600 & -3.11566500 & -5.14406400 \\
\hline $\mathrm{O}$ & -0.81246900 & -3.12761000 & -6.55936700 \\
\hline $\mathrm{C}$ & -0.44097000 & -1.69927700 & -4.66176200 \\
\hline $\mathrm{S}$ & -0.64296100 & -1.42062400 & -2.93554900 \\
\hline $\mathrm{C}$ & -0.27015400 & -0.64147100 & -5.50041400 \\
\hline $\mathrm{S}$ & -0.23272700 & 1.01491800 & -4.86962100 \\
\hline $\mathrm{C}$ & -0.12569100 & -0.81969200 & -7.00292100 \\
\hline $\mathrm{C}$ & 0.12642400 & -2.29958200 & -7.32276000 \\
\hline $\mathrm{C}$ & -3.10627100 & 4.91369700 & -2.32652900 \\
\hline
\end{tabular}




\begin{tabular}{|c|c|c|c|}
\hline $\mathrm{O}$ & -4.57064200 & 4.97309000 & -2.33375900 \\
\hline $\mathrm{C}$ & -2.57776700 & 3.49771500 & -2.16442600 \\
\hline $\mathrm{S}$ & -0.93530600 & 3.20654000 & -2.77632100 \\
\hline $\mathrm{C}$ & -3.34032600 & 2.48771600 & -1.66755700 \\
\hline $\mathrm{S}$ & -2.60816200 & 0.85430500 & -1.48648600 \\
\hline $\mathrm{C}$ & -4.80664900 & 2.69996400 & -1.32405500 \\
\hline $\mathrm{C}$ & -5.13815600 & 4.20836900 & -1.22115900 \\
\hline $\mathrm{W}$ & -0.41940200 & 0.89680900 & -2.51088300 \\
\hline $\mathrm{O}$ & -3.97024000 & -1.90514600 & -0.75478100 \\
\hline $\mathrm{O}$ & 0.55590700 & -5.21884800 & -2.72938700 \\
\hline $\mathrm{O}$ & -0.58528000 & -3.90267600 & 0.67332500 \\
\hline $\mathrm{O}$ & -2.08691300 & -3.40785800 & -1.25564000 \\
\hline $\mathrm{O}$ & 0.49226500 & 0.25045600 & -0.70651300 \\
\hline $\mathrm{H}$ & -8.43144200 & -1.03072700 & -5.17968600 \\
\hline $\mathrm{H}$ & -8.23401500 & -4.69086800 & -3.03256000 \\
\hline $\mathrm{H}$ & -6.23980800 & -5.37709800 & -1.70378400 \\
\hline $\mathrm{H}$ & -5.66128800 & -4.16304000 & 0.40476000 \\
\hline $\mathrm{H}$ & -7.05180000 & -2.23801700 & 1.15031000 \\
\hline $\mathrm{H}$ & -9.07964100 & -1.57945200 & -0.16443400 \\
\hline $\mathrm{H}$ & -10.84015500 & -2.36034300 & -1.81626000 \\
\hline $\mathrm{H}$ & -10.41228500 & -3.55106600 & -3.05587800 \\
\hline $\mathrm{H}$ & -11.50581400 & -1.75999800 & -4.70285600 \\
\hline $\mathrm{H}$ & -11.08697400 & -0.12439000 & -4.43945100 \\
\hline $\mathrm{H}$ & -9.35759500 & -0.67246000 & -2.93885700 \\
\hline $\mathrm{H}$ & 1.16030700 & -2.58301600 & -7.07327100 \\
\hline $\mathrm{H}$ & -0.07225200 & -2.53168200 & -8.37387400 \\
\hline $\mathrm{H}$ & -1.04219700 & -0.47175700 & -7.50118500 \\
\hline $\mathrm{H}$ & 0.70194200 & -0.20408000 & -7.38363000 \\
\hline $\mathrm{H}$ & -1.39190300 & -3.63619400 & -4.67900200 \\
\hline $\mathrm{H}$ & 0.37155100 & -3.68487100 & -4.91861600 \\
\hline $\mathrm{H}$ & -2.72175900 & 5.55782400 & -1.51670400 \\
\hline $\mathrm{H}$ & -2.79330500 & 5.33491200 & -3.28691500 \\
\hline $\mathrm{H}$ & -5.06167800 & 2.21982100 & -0.36646600 \\
\hline $\mathrm{H}$ & -5.43262200 & 2.23065300 & -2.09981400 \\
\hline $\mathrm{H}$ & -4.75722200 & 4.61098400 & -0.27125000 \\
\hline $\mathrm{H}$ & -6.21919400 & 4.37895000 & -1.27682600 \\
\hline $\mathrm{H}$ & 3.70451800 & 8.53217000 & -0.13227400 \\
\hline $\mathrm{H}$ & 7.31521400 & 6.27560300 & -3.89821000 \\
\hline $\mathrm{H}$ & 5.30106400 & 4.89392600 & -4.43525500 \\
\hline $\mathrm{H}$ & 3.54996400 & 4.46432800 & -2.72043700 \\
\hline $\mathrm{H}$ & 3.73585300 & 5.42915900 & -0.44400900 \\
\hline $\mathrm{H}$ & 8.48710600 & 7.73423400 & -1.72438600 \\
\hline $\mathrm{H}$ & 7.93949800 & 8.28037800 & 0.69821500 \\
\hline $\mathrm{H}$ & 4.68198300 & 6.28725800 & 1.77967300 \\
\hline $\mathrm{H}$ & 6.07741900 & 7.07402900 & 2.51291200 \\
\hline $\mathrm{H}$ & 3.71397200 & 7.60142000 & 3.66665700 \\
\hline $\mathrm{H}$ & 3.43161400 & 9.24165900 & 3.53289700 \\
\hline $\mathrm{H}$ & 5.23770600 & 9.29808600 & 1.77963600 \\
\hline $\mathrm{H}$ & 5.96458600 & -5.33928300 & 8.93227600 \\
\hline $\mathrm{H}$ & 2.84727500 & -5.53599400 & 7.75089400 \\
\hline
\end{tabular}




\begin{tabular}{|c|c|c|c|}
\hline $\mathrm{H}$ & 3.07534500 & -4.77647100 & 9.23670400 \\
\hline $\mathrm{H}$ & 4.79541500 & -4.63424200 & 6.82680300 \\
\hline $\mathrm{H}$ & 3.43742400 & -2.42220300 & 8.44864000 \\
\hline $\mathrm{H}$ & 4.90824800 & -2.09296600 & 7.56458300 \\
\hline $\mathrm{H}$ & 3.64117200 & -4.12710200 & 4.85822200 \\
\hline $\mathrm{H}$ & 2.19383600 & -2.81205100 & 3.23576200 \\
\hline $\mathrm{H}$ & 0.74120700 & -0.34773200 & 3.20017000 \\
\hline $\mathrm{H}$ & 0.15213500 & 1.57112500 & 4.67868100 \\
\hline $\mathrm{H}$ & 0.98939800 & 1.68624900 & 7.01527700 \\
\hline $\mathrm{H}$ & 2.44533100 & -0.11368600 & 7.94191500 \\
\hline $\mathrm{H}$ & 2.51723300 & -0.46855800 & -3.07666100 \\
\hline $\mathrm{H}$ & 2.42209600 & 0.56443800 & -4.52101200 \\
\hline $\mathrm{H}$ & 5.84594800 & -0.32690800 & -4.70812200 \\
\hline $\mathrm{H}$ & 4.25446900 & -0.66753300 & -5.22312600 \\
\hline $\mathrm{H}$ & 4.61592900 & 1.63490900 & -3.80364200 \\
\hline $\mathrm{H}$ & 5.99729300 & 2.04183500 & -2.13683200 \\
\hline $\mathrm{H}$ & 7.06031400 & 2.03305000 & -0.13922300 \\
\hline $\mathrm{H}$ & 4.80665900 & 0.21942900 & 0.85821300 \\
\hline $\mathrm{H}$ & 6.81077500 & 0.32814500 & 2.42676100 \\
\hline $\mathrm{H}$ & 6.71489400 & 2.09529800 & 2.40344900 \\
\hline $\mathrm{H}$ & 5.39691900 & 1.13876800 & 3.11104300 \\
\hline $\mathrm{H}$ & 3.63881200 & 2.52595400 & 2.82240000 \\
\hline $\mathrm{H}$ & 2.57019700 & 3.16116400 & 1.55677100 \\
\hline $\mathrm{H}$ & 2.73350500 & 1.41476100 & 1.78221600 \\
\hline $\mathrm{H}$ & 5.02075500 & 3.27952200 & 0.84766900 \\
\hline $\mathrm{H}$ & 3.99577600 & 2.33000000 & -0.23465700 \\
\hline $\mathrm{H}$ & 7.06377600 & -0.99559600 & -0.64813400 \\
\hline $\mathrm{H}$ & -4.01108200 & 1.07437400 & 4.17363400 \\
\hline $\mathrm{H}$ & -6.29972200 & -0.34122600 & 3.09631600 \\
\hline $\mathrm{H}$ & -5.06317800 & -0.27275000 & 1.92227800 \\
\hline $\mathrm{H}$ & -4.91986500 & -1.78896400 & 4.47883200 \\
\hline $\mathrm{H}$ & -2.67685200 & -2.36818200 & 3.91944300 \\
\hline $\mathrm{H}$ & -3.65004800 & -2.66884000 & 2.46662400 \\
\hline $\mathrm{H}$ & -9.52162400 & 7.67225500 & 4.11649700 \\
\hline $\mathrm{H}$ & -8.51738900 & 5.53756300 & 1.61200000 \\
\hline $\mathrm{H}$ & -8.46066500 & 7.20779700 & 1.47651800 \\
\hline $\mathrm{H}$ & -7.97752400 & 5.55156700 & 3.89005800 \\
\hline $\mathrm{H}$ & -6.11448400 & 7.49943100 & 2.44154700 \\
\hline $\mathrm{H}$ & -5.89518900 & 7.11334500 & 4.13778700 \\
\hline $\mathrm{H}$ & -6.80684000 & 3.69715900 & 3.04006200 \\
\hline $\mathrm{H}$ & -4.75211400 & 2.34078900 & 2.35870900 \\
\hline $\mathrm{H}$ & -2.05208700 & 2.80070900 & 1.61731200 \\
\hline $\mathrm{H}$ & -0.42999900 & 4.67317000 & 1.29892900 \\
\hline $\mathrm{H}$ & -1.09813500 & 7.03175800 & 1.70717300 \\
\hline $\mathrm{H}$ & -3.41849100 & 7.59935400 & 2.42158500 \\
\hline $\mathrm{H}$ & 6.27637000 & -4.06633500 & -4.99919700 \\
\hline $\mathrm{H}$ & 0.71993900 & -5.11721600 & -6.62207300 \\
\hline $\mathrm{H}$ & 1.99741500 & -5.01724200 & -7.84320200 \\
\hline $\mathrm{H}$ & 1.21233400 & -6.58088500 & -7.49769200 \\
\hline $\mathrm{H}$ & 1.49609300 & -6.31436300 & -4.23854100 \\
\hline
\end{tabular}




$\begin{array}{lrrr}\mathrm{H} & 1.96230200 & -7.72882300 & -5.05795500 \\ \mathrm{H} & 3.43896000 & -6.55926900 & -6.49118600 \\ \mathrm{H} & 3.73631700 & -5.74735600 & -3.78623500 \\ \mathrm{H} & 4.25054900 & -2.81501900 & -3.89827100 \\ \mathrm{H} & 4.65758800 & -2.79934000 & -1.62619200 \\ \mathrm{H} & 3.32947100 & -3.92463000 & -1.82291700 \\ \mathrm{H} & 4.94190400 & -5.88385000 & -1.51539700 \\ \mathrm{H} & 6.26116100 & -4.71773000 & -1.28043700 \\ \mathrm{H} & 5.33376700 & -3.12216900 & 2.19950400 \\ \mathrm{H} & 6.60274800 & -3.27451200 & 0.95324300 \\ \mathrm{H} & 5.07794000 & -2.40725700 & 0.58143800 \\ \mathrm{H} & -2.77503300 & -6.25119000 & 3.09048600 \\ \mathrm{H} & -0.57949300 & -8.41869300 & 2.87268200 \\ \mathrm{H} & -0.15284700 & -6.77628400 & 2.80060500 \\ \mathrm{H} & -1.45020200 & -8.18733600 & 5.09350100 \\ \mathrm{H} & 0.21841100 & -6.45643300 & 5.64534800 \\ \mathrm{H} & -1.74310500 & -4.36164300 & 4.51935000 \\ \mathrm{H} & -0.18228200 & -4.04917500 & 5.29193600 \\ \mathrm{H} & -0.23026000 & -4.82926000 & 3.71191200 \\ \mathrm{H} & -2.04979300 & -8.10476400 & 7.22714200 \\ \mathrm{H} & -0.49181800 & -7.52711200 & 7.85754500 \\ \mathrm{H} & -2.01191800 & -7.02237800 & 8.62357800 \\ \mathrm{H} & -1.20408700 & -5.13277400 & 7.26843200 \\ \mathrm{H} & -2.65634900 & -5.77574400 & 6.51182400 \\ \mathrm{H} & -4.92352300 & -2.10723400 & -0.82945200 \\ \mathrm{H} & -3.72866900 & -0.95741000 & -0.99988600 \\ \mathrm{H} & -2.96649600 & -2.85962000 & -1.00242100 \\ \mathrm{H} & -1.58589000 & -2.86097300 & -1.97393200 \\ \mathrm{H} & -1.45530100 & -3.71037300 & -0.45589200 \\ \mathrm{H} & -1.00146800 & -3.31396600 & 1.36290000 \\ \mathrm{H} & -0.38694600 & -5.22149700 & -2.46997100 \\ \mathrm{H} & 0.96974700 & -4.38039300 & -2.43701500 \\ \mathrm{H} & 0.53355800 & -0.78480300 & -0.18070500 \\ \mathrm{H} & 1.37432500 & 0.70407300 & -0.65649000 \\ \mathrm{C} & -0.52476300 & -1.31248500 & 1.28777400 \\ \mathrm{C} & 1.03963000 & -2.06580800 & 0.33575100 \\ \mathrm{H} & 0.77870600 & -3.38513400 & 0.44105300 \\ \mathrm{H} & 2.11753500 & -1.89104400 & 0.18870900 \\ & 1.47298900 & -4.22227000 & 0.39409600\end{array}$

XE-EP1,$E=-5430.279127$

$\begin{array}{lrrc}\mathrm{N} & -5.29701400 & -1.08943100 & 2.98277100 \\ \mathrm{C} & -4.04104500 & -1.20687900 & 3.76259300 \\ \mathrm{C} & -3.75959900 & -0.03658500 & 4.73359200 \\ \mathrm{O} & -3.38632200 & -0.21217500 & 5.90818100 \\ \mathrm{C} & -2.83440200 & -1.36385500 & 2.81704900 \\ \mathrm{C} & -2.50402200 & -0.10017900 & 2.04927900 \\ \mathrm{O} & -3.23696500 & 0.90902700 & 1.96819100 \\ \mathrm{O} & -1.29349200 & -0.20418400 & 1.43914300 \\ \mathrm{~N} & 0.31606300 & -7.39044700 & 3.91033600 \\ & & & \mathrm{~S} 50\end{array}$




\begin{tabular}{|c|c|c|c|}
\hline $\mathrm{C}$ & -0.60079800 & -6.93679900 & 4.97057100 \\
\hline $\mathrm{C}$ & -2.05986100 & -6.95191900 & 4.49890500 \\
\hline $\mathrm{O}$ & -3.01507300 & -7.40368900 & 5.16068400 \\
\hline $\mathrm{C}$ & -0.20616500 & -5.51541200 & 5.48878500 \\
\hline $\mathrm{C}$ & -0.79829600 & -5.22330200 & 6.89317700 \\
\hline $\mathrm{C}$ & -0.59573000 & -4.38924800 & 4.49829500 \\
\hline $\mathrm{C}$ & -0.15499700 & -6.03428500 & 8.03853400 \\
\hline $\mathrm{N}$ & 2.79754400 & -6.96859000 & -4.42918000 \\
\hline $\mathrm{C}$ & 3.03687100 & -6.13225100 & -5.62857400 \\
\hline $\mathrm{C}$ & 3.59968500 & -4.75803000 & -5.17843600 \\
\hline $\mathrm{O}$ & 3.49981900 & -3.70697400 & -5.87965800 \\
\hline $\mathrm{C}$ & 1.85210500 & -5.99162700 & -6.60589000 \\
\hline $\mathrm{N}$ & 4.25305500 & -4.80198000 & -3.97621100 \\
\hline $\mathrm{C}$ & 4.83045500 & -3.61527700 & -3.32777500 \\
\hline $\mathrm{C}$ & 6.26972000 & -3.41772300 & -3.80474000 \\
\hline $\mathrm{O}$ & 7.19601700 & -2.92204200 & -3.13753300 \\
\hline $\mathrm{C}$ & 4.63376100 & -3.58923100 & -1.79230400 \\
\hline $\mathrm{C}$ & 5.55763500 & -4.50936300 & -0.96630800 \\
\hline $\mathrm{S}$ & 5.21142500 & -4.44438100 & 0.85951500 \\
\hline $\mathrm{C}$ & 5.80167000 & -2.73297400 & 1.24324500 \\
\hline $\mathrm{N}$ & 4.77729100 & -0.29166800 & -4.50846700 \\
\hline $\mathrm{C}$ & 4.18462800 & 0.70255500 & -3.60808700 \\
\hline $\mathrm{C}$ & 4.81152200 & 0.54357300 & -2.18173900 \\
\hline $\mathrm{O}$ & 4.56194700 & -0.45402200 & -1.45132000 \\
\hline $\mathrm{C}$ & 2.67055400 & 0.46359900 & -3.52529000 \\
\hline $\mathrm{S}$ & 1.78947200 & 1.74459600 & -2.49313800 \\
\hline $\mathrm{N}$ & 5.66853800 & 1.54069200 & -1.77100400 \\
\hline $\mathrm{C}$ & 6.31766200 & 1.61966800 & -0.43202400 \\
\hline $\mathrm{C}$ & 7.36787000 & 0.50786800 & -0.27380000 \\
\hline $\mathrm{O}$ & 8.46798500 & 0.69154100 & 0.27954700 \\
\hline $\mathrm{C}$ & 5.30096400 & 1.63333600 & 0.77048100 \\
\hline $\mathrm{C}$ & 4.28168400 & 2.78735400 & 0.58135200 \\
\hline $\mathrm{C}$ & 6.05576900 & 1.75797300 & 2.11437100 \\
\hline $\mathrm{C}$ & 3.14976900 & 2.79614700 & 1.63077800 \\
\hline $\mathrm{N}$ & -9.10634300 & 5.71902800 & 1.99573200 \\
\hline $\mathrm{C}$ & -8.21744100 & 6.04335700 & 3.14120000 \\
\hline $\mathrm{C}$ & -8.97667200 & 7.00653800 & 4.07132500 \\
\hline $\mathrm{O}$ & -8.45526400 & 7.87080400 & 4.79699200 \\
\hline $\mathrm{C}$ & -6.79820600 & 6.57956800 & 2.81948300 \\
\hline $\mathrm{C}$ & -5.73256600 & 5.56367600 & 2.47088400 \\
\hline $\mathrm{C}$ & -5.73800000 & 4.17644300 & 2.56037100 \\
\hline $\mathrm{C}$ & -4.40191200 & 5.93513100 & 2.01995300 \\
\hline $\mathrm{N}$ & -4.48606500 & 3.66539100 & 2.18840700 \\
\hline $\mathrm{C}$ & -3.65191700 & 4.72942100 & 1.84912500 \\
\hline $\mathrm{C}$ & -3.77989900 & 7.17941700 & 1.75036200 \\
\hline $\mathrm{C}$ & -2.31616000 & 4.73221700 & 1.40042000 \\
\hline $\mathrm{C}$ & -2.44835900 & 7.19043700 & 1.31412300 \\
\hline $\mathrm{C}$ & -1.72658500 & 5.97613000 & 1.13594900 \\
\hline $\mathrm{N}$ & 3.40053400 & 8.94705200 & 2.77929000 \\
\hline$r$ & 3.81759100 & 8.87378300 & 1.37396400 \\
\hline
\end{tabular}




\begin{tabular}{|c|c|c|c|}
\hline C & 2.65468500 & 8.89285700 & 0.37070000 \\
\hline J & 1.46332500 & 9.04613700 & 0.70321400 \\
\hline $\mathrm{C}$ & 4.73907900 & 7.63012100 & 1.15808900 \\
\hline $\mathrm{C}$ & 5.42352600 & 7.55991300 & -0.18612900 \\
\hline $\mathrm{C}$ & 6.58036900 & 8.23101000 & -0.55330700 \\
\hline $\mathrm{C}$ & 5.02056600 & 6.77178300 & -1.34052400 \\
\hline $\mathrm{N}$ & 6.91856800 & 7.91019500 & -1.87280200 \\
\hline $\mathrm{C}$ & 5.97753500 & 7.01025500 & -2.37888800 \\
\hline $\mathrm{C}$ & 3.95142200 & 5.87604600 & -1.58720000 \\
\hline $\mathrm{C}$ & 5.89198700 & 6.38839900 & -3.63945000 \\
\hline $\mathrm{C}$ & 3.85876700 & 5.24943800 & -2.83756200 \\
\hline $\mathrm{C}$ & 4.82093700 & 5.50629600 & -3.85429500 \\
\hline $\mathrm{N}$ & 4.12995100 & -4.31851100 & 8.65230800 \\
\hline $\mathrm{C}$ & 5.02242900 & -3.40170000 & 7.92507200 \\
\hline $\mathrm{C}$ & 6.22345900 & -3.27752400 & 8.84700800 \\
\hline $\mathrm{O}$ & 6.75795800 & -2.21250600 & 9.20731300 \\
\hline $\mathrm{C}$ & 4.45069800 & -2.03151400 & 7.50278400 \\
\hline $\mathrm{C}$ & 3.54332100 & -2.05306200 & 6.29607700 \\
\hline $\mathrm{C}$ & 3.41680400 & -3.02076700 & 5.30920900 \\
\hline $\mathrm{C}$ & 2.67204800 & -0.95793800 & 5.90837500 \\
\hline $\mathrm{N}$ & 2.51520800 & -2.58582200 & 4.32831200 \\
\hline $\mathrm{C}$ & 2.04255800 & -1.31973800 & 4.67465100 \\
\hline $\mathrm{C}$ & 2.37514000 & 0.29656300 & 6.49352600 \\
\hline $\mathrm{C}$ & 1.13243300 & -0.46728700 & 4.01776100 \\
\hline $\mathrm{C}$ & 1.46968100 & 1.14803700 & 5.84684300 \\
\hline $\mathrm{C}$ & 0.85614800 & 0.76838000 & 4.62123200 \\
\hline $\mathrm{N}$ & -10.99158400 & -2.34148800 & -3.68502000 \\
\hline $\mathrm{C}$ & -9.69144000 & -2.65054500 & -3.05728600 \\
\hline $\mathrm{C}$ & -8.77573300 & -2.99763700 & -4.23065400 \\
\hline $\mathrm{O}$ & -8.54280900 & -4.15935200 & -4.61927800 \\
\hline $\mathrm{C}$ & -9.83021200 & -3.77849500 & -2.01004500 \\
\hline $\mathrm{C}$ & -8.55737400 & -4.03793500 & -1.21518700 \\
\hline $\mathrm{C}$ & -8.22245200 & -3.22575300 & -0.10763500 \\
\hline $\mathrm{C}$ & -7.68469500 & -5.09241800 & -1.56768800 \\
\hline $\mathrm{C}$ & -7.04959600 & -3.46126800 & 0.63726400 \\
\hline $\mathrm{C}$ & -6.51677400 & -5.33907000 & -0.82364100 \\
\hline $\mathrm{C}$ & -6.19718300 & -4.52807300 & 0.28331000 \\
\hline $\mathrm{C}$ & -0.45748700 & -3.50832000 & -4.85300700 \\
\hline $\mathrm{O}$ & -0.67556700 & -3.64301400 & -6.27562300 \\
\hline $\mathrm{C}$ & -0.45139400 & -2.05637900 & -4.46340000 \\
\hline $\mathrm{S}$ & -0.69522600 & -1.68124000 & -2.75777200 \\
\hline $\mathrm{C}$ & -0.35115600 & -1.04901700 & -5.37220000 \\
\hline $\mathrm{S}$ & -0.39795800 & 0.64221900 & -4.83738000 \\
\hline $\mathrm{C}$ & -0.19651700 & -1.31380100 & -6.86040900 \\
\hline $\mathrm{C}$ & 0.20091000 & -2.78184100 & -7.07617500 \\
\hline $\mathrm{C}$ & -3.58107100 & 4.44382900 & -2.49794400 \\
\hline $\mathrm{O}$ & -5.04449800 & 4.35079500 & -2.45299400 \\
\hline $\mathrm{C}$ & -2.90215000 & 3.10596800 & -2.23757100 \\
\hline $\mathrm{S}$ & -1.25839100 & 2.89825600 & -2.87763300 \\
\hline $\mathrm{C}$ & -3.55037600 & 2.07573400 & -1.62957700 \\
\hline
\end{tabular}




\begin{tabular}{|c|c|c|c|}
\hline S & -2.68363400 & 0.52263900 & -1.38972600 \\
\hline $\mathrm{C}$ & -5.01405600 & 2.17443600 & -1.22937300 \\
\hline $\mathrm{C}$ & -5.49797600 & 3.64396800 & -1.25322500 \\
\hline $\mathrm{W}$ & -0.55450900 & 0.64688700 & -2.49108300 \\
\hline $\mathrm{O}$ & -3.81858900 & -2.34715700 & -0.22265100 \\
\hline $\mathrm{O}$ & 0.62821700 & -6.20612800 & -2.56565700 \\
\hline $\mathrm{O}$ & 0.33971800 & -4.96496600 & -0.10140200 \\
\hline $\mathrm{O}$ & -1.80767000 & -3.92138100 & -0.80286200 \\
\hline $\mathrm{O}$ & 0.51206800 & 0.09602000 & -0.58540500 \\
\hline $\mathrm{H}$ & -8.40665500 & -2.12439700 & -4.80709800 \\
\hline $\mathrm{H}$ & -7.91928300 & -5.70691100 & -2.43317300 \\
\hline $\mathrm{H}$ & -5.85671700 & -6.15579700 & -1.10540600 \\
\hline $\mathrm{H}$ & -5.30484700 & -4.73172900 & 0.87071000 \\
\hline $\mathrm{H}$ & -6.79558700 & -2.83398600 & 1.48943200 \\
\hline $\mathrm{H}$ & -8.88927100 & -2.41421800 & 0.18252000 \\
\hline $\mathrm{H}$ & -10.64919800 & -3.48300900 & -1.34295000 \\
\hline $\mathrm{H}$ & -10.13838300 & -4.69507300 & -2.52924700 \\
\hline $\mathrm{H}$ & -11.40795600 & -3.08950500 & -4.23070600 \\
\hline $\mathrm{H}$ & -11.11607200 & -1.41390800 & -4.07494500 \\
\hline $\mathrm{H}$ & -9.32828500 & -1.73649000 & -2.57184400 \\
\hline $\mathrm{H}$ & 1.25052700 & -2.95080800 & -6.79397700 \\
\hline $\mathrm{H}$ & 0.04476600 & -3.09798500 & -8.11241300 \\
\hline $\mathrm{H}$ & -1.14432600 & -1.09668300 & -7.37400400 \\
\hline $\mathrm{H}$ & 0.56548000 & -0.64872000 & -7.29173300 \\
\hline $\mathrm{H}$ & -1.28896900 & -4.04154200 & -4.37570800 \\
\hline $\mathrm{H}$ & 0.48522900 & -3.99703700 & -4.55362300 \\
\hline $\mathrm{H}$ & -3.24200900 & 5.19298800 & -1.76279700 \\
\hline $\mathrm{H}$ & -3.34389000 & 4.80619200 & -3.50288100 \\
\hline $\mathrm{H}$ & -5.16852300 & 1.76379500 & -0.22054600 \\
\hline $\mathrm{H}$ & -5.61770800 & 1.56913800 & -1.92349200 \\
\hline $\mathrm{H}$ & -5.13851400 & 4.17505200 & -0.36178500 \\
\hline $\mathrm{H}$ & -6.59171000 & 3.69663200 & -1.28556900 \\
\hline $\mathrm{H}$ & 2.93499400 & 8.78929000 & -0.69429000 \\
\hline $\mathrm{H}$ & 6.62315900 & 6.58161800 & -4.42149400 \\
\hline $\mathrm{H}$ & 4.71562300 & 5.01531100 & -4.81920100 \\
\hline $\mathrm{H}$ & 3.04588900 & 4.55400900 & -3.03163500 \\
\hline $\mathrm{H}$ & 3.20855600 & 5.66560500 & -0.82133900 \\
\hline $\mathrm{H}$ & 7.72502800 & 8.25772100 & -2.37087300 \\
\hline $\mathrm{H}$ & 7.19386800 & 8.90285100 & 0.03063100 \\
\hline $\mathrm{H}$ & 4.14493300 & 6.71951600 & 1.32397700 \\
\hline $\mathrm{H}$ & 5.48062000 & 7.67191100 & 1.96522700 \\
\hline $\mathrm{H}$ & 3.05238700 & 8.05653400 & 3.13451700 \\
\hline $\mathrm{H}$ & 2.71227500 & 9.67942700 & 2.95099800 \\
\hline $\mathrm{H}$ & 4.42646200 & 9.76277600 & 1.13780700 \\
\hline $\mathrm{H}$ & 6.57824700 & -4.25611100 & 9.22420900 \\
\hline 11 & 3.49104600 & -4.85435900 & 8.07152500 \\
\hline $\mathrm{H}$ & 3.65203400 & -3.89119200 & 9.44272600 \\
\hline $\mathrm{H}$ & 5.38489300 & -3.94398700 & 7.03724500 \\
\hline$\Pi$ & 3.91686600 & -1.59980700 & 8.36320900 \\
\hline$\pi$ & 29668000 & -1.35263000 & 7.31934 \\
\hline
\end{tabular}




\begin{tabular}{|c|c|c|c|}
\hline $\mathrm{H}$ & 3.90158700 & -3.98183700 & 5.21032000 \\
\hline $\mathrm{H}$ & 2.24013200 & -3.10734300 & 3.50511100 \\
\hline $\mathrm{H}$ & 0.65834600 & -0.73968200 & 3.07943300 \\
\hline $\mathrm{H}$ & 0.16117300 & 1.45325600 & 4.14059200 \\
\hline $\mathrm{H}$ & 1.22618500 & 2.11385900 & 6.28304000 \\
\hline $\mathrm{H}$ & 2.84294100 & 0.59867000 & 7.42803900 \\
\hline $\mathrm{H}$ & 2.48833900 & -0.53749800 & -3.12139700 \\
\hline $\mathrm{H}$ & 2.24405400 & 0.51687100 & -4.53164900 \\
\hline $\mathrm{H}$ & 5.72046400 & -0.12999700 & -4.83914300 \\
\hline $\mathrm{H}$ & 4.15853600 & -0.75659200 & -5.16356600 \\
\hline $\mathrm{H}$ & 4.35931600 & 1.73983500 & -3.95108500 \\
\hline $\mathrm{H}$ & 5.76437200 & 2.35295900 & -2.37122000 \\
\hline $\mathrm{H}$ & 6.85961000 & 2.57534000 & -0.40446000 \\
\hline $\mathrm{H}$ & 4.75210800 & 0.68319600 & 0.75296800 \\
\hline $\mathrm{H}$ & 6.76337200 & 0.93793800 & 2.27624200 \\
\hline $\mathrm{H}$ & 6.62399400 & 2.69822600 & 2.16123100 \\
\hline $\mathrm{H}$ & 5.34547200 & 1.74929000 & 2.94825900 \\
\hline $\mathrm{H}$ & 3.51489400 & 3.02009500 & 2.64050100 \\
\hline $\mathrm{H}$ & 2.39277500 & 3.54928900 & 1.37341500 \\
\hline $\mathrm{H}$ & 2.64338400 & 1.82218000 & 1.67185000 \\
\hline $\mathrm{H}$ & 4.82219800 & 3.74825400 & 0.61079400 \\
\hline $\mathrm{H}$ & 3.82592100 & 2.70803100 & -0.41242900 \\
\hline $\mathrm{H}$ & 7.08834700 & -0.47617800 & -0.68624100 \\
\hline $\mathrm{H}$ & -3.93748900 & 0.97824700 & 4.33361900 \\
\hline $\mathrm{H}$ & -6.13759900 & -1.07681400 & 3.55730000 \\
\hline $\mathrm{H}$ & -5.27479600 & -0.33344600 & 2.30012900 \\
\hline $\mathrm{H}$ & -4.10017100 & -2.11396100 & 4.37605100 \\
\hline $\mathrm{H}$ & -1.94325100 & -1.67867600 & 3.37053900 \\
\hline $\mathrm{H}$ & -3.06211900 & -2.13344900 & 2.06739000 \\
\hline $\mathrm{H}$ & -10.07423200 & 6.85343700 & 4.04648600 \\
\hline $\mathrm{H}$ & -8.88676300 & 4.83366300 & 1.54611100 \\
\hline $\mathrm{H}$ & -9.17085000 & 6.47057800 & 1.31041800 \\
\hline $\mathrm{H}$ & -8.12342200 & 5.11721100 & 3.73429600 \\
\hline $\mathrm{H}$ & -6.88938100 & 7.30733100 & 1.99664100 \\
\hline $\mathrm{H}$ & -6.46267100 & 7.16223900 & 3.69009400 \\
\hline $\mathrm{H}$ & -6.53107700 & 3.50855900 & 2.86796600 \\
\hline $\mathrm{H}$ & -4.20956700 & 2.68841200 & 2.13385600 \\
\hline $\mathrm{H}$ & -1.77023800 & 3.80554000 & 1.24682700 \\
\hline $\mathrm{H}$ & -0.70031900 & 6.01943200 & 0.77988400 \\
\hline $\mathrm{H}$ & -1.94952500 & 8.13285800 & 1.10143000 \\
\hline $\mathrm{H}$ & -4.32315300 & 8.11319800 & 1.87968200 \\
\hline $\mathrm{H}$ & 6.43677400 & -3.73891100 & -4.85332000 \\
\hline $\mathrm{H}$ & 0.96917200 & -5.56043100 & -6.12588100 \\
\hline $\mathrm{H}$ & 2.12246400 & -5.34469900 & -7.44808200 \\
\hline $\mathrm{H}$ & 1.58204000 & -6.98269100 & -6.99495300 \\
\hline $\mathrm{H}$ & 2.00924000 & -6.67611100 & -3.83175900 \\
\hline $\mathrm{H}$ & 2.77663100 & -7.96550100 & -4.62781800 \\
\hline $\mathrm{H}$ & 3.86629900 & -6.60499400 & -6.18101000 \\
\hline 11 & 4.17750600 & -5.70230300 & $-3.4978630 \mathrm{c}$ \\
\hline 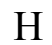 & 30521400 & -2.73619200 & -3.73499 \\
\hline
\end{tabular}




$\begin{array}{lrrr}\mathrm{H} & 4.77254600 & -2.55273200 & -1.48075700 \\ \mathrm{H} & 3.58445500 & -3.84051600 & -1.59285300 \\ \mathrm{H} & 5.42663300 & -5.56969100 & -1.22865700 \\ \mathrm{H} & 6.60942100 & -4.24947300 & -1.12034800 \\ \mathrm{H} & 5.62315300 & -2.56653000 & 2.31178300 \\ \mathrm{H} & 6.87515600 & -2.64936200 & 1.04020300 \\ \mathrm{H} & 5.25273300 & -1.98682600 & 0.66153500 \\ \mathrm{H} & -2.21761600 & -6.56218900 & 3.47081500 \\ \mathrm{H} & 0.22093500 & -8.35976000 & 3.62409100 \\ \mathrm{H} & 0.46095500 & -6.75326400 & 3.13167200 \\ \mathrm{H} & -0.52344900 & -7.63975200 & 5.80829000 \\ \mathrm{H} & 0.89117500 & -5.53906200 & 5.57265700 \\ \mathrm{H} & -1.68554800 & -4.25097200 & 4.46382100 \\ \mathrm{H} & -0.15362800 & -3.44156500 & 4.82531700 \\ \mathrm{H} & -0.24549700 & -4.57967300 & 3.47458300 \\ \mathrm{H} & -0.31396000 & -7.11381600 & 7.92103500 \\ \mathrm{H} & 0.92968600 & -5.85820200 & 8.08196600 \\ \mathrm{H} & -0.58177500 & -5.74386800 & 9.00754500 \\ \mathrm{H} & -0.66288500 & -4.15229000 & 7.10101700 \\ \mathrm{H} & -1.88375600 & -5.40183700 & 6.88085900 \\ \mathrm{H} & -4.76501600 & -2.59176200 & -0.24302500 \\ \mathrm{H} & -3.64971800 & -1.46494700 & -0.64508600 \\ \mathrm{H} & -2.62593700 & -3.46184100 & -0.44404200 \\ \mathrm{H} & -1.50843700 & -3.39994800 & -1.59033000 \\ \mathrm{H} & -0.61431300 & -4.55281500 & -0.23927500 \\ \mathrm{H} & -1.11008000 & 0.45416900 & 0.71691400 \\ \mathrm{H} & -0.29293700 & -6.20072800 & -2.88679100 \\ \mathrm{H} & 0.66049300 & -5.82790000 & -1.64484100 \\ \mathrm{H} & 0.02707400 & -2.49909800 & 1.14355600 \\ \mathrm{H} & 0.59551000 & -0.84258300 & -0.30405100 \\ \mathrm{H} & 1.39939500 & 0.56089700 & -0.73042300 \\ \mathrm{C} & 1.02855800 & -2.90414400 & 1.01913500 \\ \mathrm{C} & 1.27308200 & -4.10697600 & 0.43827300 \\ \mathrm{H} & 1.87177100 & -2.31946800 & 1.37690100 \\ \mathrm{H} & 2.28687600 & -4.49615200 & 0.36603500 \\ & & & \\ & & & \\ & & & \end{array}$

XE-EP2,$E=-5430.277941$

$\mathrm{N}$
$\mathrm{C}$
$\mathrm{C}$
$\mathrm{O}$
$\mathrm{C}$
$\mathrm{C}$
$\mathrm{O}$
$\mathrm{O}$
$\mathrm{N}$
$\mathrm{C}$
$\mathrm{C}$
$\mathrm{O}$
$\mathrm{C}$

$-4.82431400-2.37994500$

3.23026700

$-3.57825200-2.03350100$

3.94739900

C $\quad-3.66446600 \quad-0.82021300$

4.89141800

$\begin{array}{lll}\mathrm{O} & -3.05817900 & -0.75914200\end{array}$

5.97746700

$-2.44192000-1.73104900$

2.95526400

C $\quad-2.68385500 \quad-0.47444200$

2.14984700

$\begin{array}{ll}-3.77985000 & 0.12392000\end{array}$

2.05104200

$\mathrm{O} \quad-1.55623000-0.07355200$

1.51365800

$2.39784200-6.68386600$

4.40782500

$1.28762300-6.12036000$

5.20682100

$-0.00132700-6.93023100$

5.01364900

$-0.72443100-7.34878600$

5.93833600

$1.05802100 \quad-4.61219400$

4.86867200 


\begin{tabular}{|c|c|c|c|}
\hline $\mathrm{C}$ & 0.34332200 & -3.85986200 & 6.02223600 \\
\hline $\mathrm{C}$ & 0.28995100 & -4.40993200 & 3.53784600 \\
\hline $\mathrm{C}$ & 1.22220000 & -3.63177800 & 7.27093700 \\
\hline $\mathrm{N}$ & 5.37698800 & -6.08019900 & -4.27137500 \\
\hline $\mathrm{C}$ & 4.61983700 & -5.24387400 & -5.23405300 \\
\hline $\mathrm{C}$ & 4.56205400 & -3.76955000 & -4.75086900 \\
\hline $\mathrm{O}$ & 3.93070600 & -2.87692400 & -5.39238500 \\
\hline $\mathrm{C}$ & 3.21244900 & -5.80177400 & -5.53723500 \\
\hline $\mathrm{N}$ & 5.26896600 & -3.51667500 & -3.60723600 \\
\hline $\mathrm{C}$ & 5.35154000 & -2.17569300 & -2.99839200 \\
\hline $\mathrm{C}$ & 6.53908700 & -1.42966000 & -3.61969400 \\
\hline $\mathrm{O}$ & 7.42052100 & -0.80479200 & -3.00271300 \\
\hline $\mathrm{C}$ & 5.30068600 & -2.16396600 & -1.45376900 \\
\hline $\mathrm{C}$ & 6.55331100 & -2.67970200 & -0.71615100 \\
\hline S & 6.46727100 & -2.49154300 & 1.13451000 \\
\hline $\mathrm{C}$ & 6.58032700 & -0.65155900 & 1.28842500 \\
\hline $\mathrm{N}$ & 4.55263600 & 0.91767900 & -4.50278500 \\
\hline $\mathrm{C}$ & 3.70260900 & 1.73245100 & -3.62039800 \\
\hline $\mathrm{C}$ & 4.37763300 & 1.83616700 & -2.21172600 \\
\hline $\mathrm{O}$ & 4.49944100 & 0.83161700 & -1.45874200 \\
\hline $\mathrm{C}$ & 2.33089100 & 1.05592900 & -3.48173800 \\
\hline $\mathrm{S}$ & 1.11836100 & 2.07691900 & -2.50234300 \\
\hline $\mathrm{N}$ & 4.85605900 & 3.07332700 & -1.84096000 \\
\hline $\mathrm{C}$ & 5.45693500 & 3.39974800 & -0.51609800 \\
\hline $\mathrm{C}$ & 6.80410600 & 2.67972900 & -0.33835900 \\
\hline $\mathrm{O}$ & 7.77342000 & 3.18998200 & 0.25403600 \\
\hline $\mathrm{C}$ & 4.49051900 & 3.14594400 & 0.70315600 \\
\hline $\mathrm{C}$ & 3.15607700 & 3.90410600 & 0.47474300 \\
\hline $\mathrm{C}$ & 5.16393900 & 3.57203100 & 2.02811100 \\
\hline $\mathrm{C}$ & 2.09816900 & 3.64821400 & 1.56937700 \\
\hline $\mathrm{N}$ & -10.47260600 & 2.93537300 & 1.93243700 \\
\hline $\mathrm{C}$ & -9.71616500 & 3.62331500 & 3.01025600 \\
\hline $\mathrm{C}$ & -10.72686600 & 4.32375400 & 3.93001900 \\
\hline $\mathrm{O}$ & -10.51396300 & 5.36124500 & 4.58154300 \\
\hline $\mathrm{C}$ & -8.57927800 & 4.58774600 & 2.58629100 \\
\hline $\mathrm{C}$ & -7.24094300 & 3.95668500 & 2.28081100 \\
\hline $\mathrm{C}$ & -6.81672000 & 2.64227900 & 2.43279000 \\
\hline $\mathrm{C}$ & -6.08908200 & 4.70128200 & 1.80399900 \\
\hline $\mathrm{N}$ & -5.46790200 & 2.52658700 & 2.07715400 \\
\hline $\mathrm{C}$ & -5.00112800 & 3.77909100 & 1.68309500 \\
\hline $\mathrm{C}$ & -5.88169300 & 6.06347200 & 1.47421200 \\
\hline $\mathrm{C}$ & -3.72863000 & 4.17842000 & 1.22691200 \\
\hline $\mathrm{C}$ & -4.61591700 & 6.46777900 & 1.02964200 \\
\hline $\mathrm{C}$ & -3.55156000 & 5.53085800 & 0.90297200 \\
\hline $\mathrm{N}$ & 0.52194500 & 9.75806600 & 2.30672200 \\
\hline $\mathrm{C}$ & 0.94598500 & 9.71191300 & 0.90218000 \\
\hline $\mathrm{C}$ & -0.17155600 & 9.32883700 & -0.07945400 \\
\hline $\mathrm{O}$ & -1.35890100 & 9.16543300 & 0.26232600 \\
\hline $\mathrm{C}$ & 2.18574600 & 8.77264800 & 0.75200800 \\
\hline $\mathrm{C}$ & 2.86575700 & 8.81384900 & -0.59578300 \\
\hline
\end{tabular}




\begin{tabular}{|c|c|c|c|}
\hline $\mathrm{C}$ & 3.76884400 & 9.77268100 & -1.03001400 \\
\hline $\mathrm{C}$ & 2.72288200 & 7.85947500 & -1.68437300 \\
\hline $\mathrm{N}$ & 4.19340800 & 9.47303300 & -2.32947600 \\
\hline $\mathrm{C}$ & 3.56818100 & 8.29802700 & -2.75400000 \\
\hline $\mathrm{C}$ & 1.97247100 & 6.66939300 & -1.84842800 \\
\hline $\mathrm{C}$ & 3.67794800 & 7.58972100 & -3.96612000 \\
\hline $\mathrm{C}$ & 2.07632700 & 5.95495000 & -3.05002200 \\
\hline $\mathrm{C}$ & 2.92146400 & 6.41421000 & -4.09915800 \\
\hline $\mathrm{N}$ & 5.13971500 & -2.35217400 & 8.90086200 \\
\hline $\mathrm{C}$ & 5.85382800 & -1.38999700 & 8.04812600 \\
\hline $\mathrm{C}$ & 6.83331900 & -0.73156400 & 8.99921200 \\
\hline $\mathrm{O}$ & 7.05350200 & 0.49030500 & 9.09197300 \\
\hline $\mathrm{C}$ & 5.00846800 & -0.36769300 & 7.25514400 \\
\hline $\mathrm{C}$ & 4.44372400 & -0.87792500 & 5.95117700 \\
\hline $\mathrm{C}$ & 5.01224400 & -1.77262700 & 5.05466700 \\
\hline $\mathrm{C}$ & 3.22060700 & -0.40150800 & 5.33330000 \\
\hline $\mathrm{N}$ & 4.20389700 & -1.88475800 & 3.91622500 \\
\hline $\mathrm{C}$ & 3.09867800 & -1.04490400 & 4.06000800 \\
\hline $\mathrm{C}$ & 2.22608100 & 0.52029500 & 5.74086100 \\
\hline $\mathrm{C}$ & 2.01821100 & -0.79336000 & 3.19223300 \\
\hline $\mathrm{C}$ & 1.15151400 & 0.77853400 & 4.88059300 \\
\hline $\mathrm{C}$ & 1.05048900 & 0.12941300 & 3.61845900 \\
\hline $\mathrm{N}$ & -9.90126100 & -5.64247100 & -3.25323400 \\
\hline $\mathrm{C}$ & -8.56668500 & -5.47497200 & -2.63991500 \\
\hline $\mathrm{C}$ & -7.59084700 & -5.64828000 & -3.80112500 \\
\hline $\mathrm{O}$ & -7.05894500 & -6.73283500 & -4.11195100 \\
\hline $\mathrm{C}$ & -8.33849800 & -6.47272300 & -1.48187600 \\
\hline $\mathrm{C}$ & -7.00061500 & -6.27971700 & -0.77732200 \\
\hline $\mathrm{C}$ & -6.81855500 & -5.23064300 & 0.15390900 \\
\hline $\mathrm{C}$ & -5.90954900 & -7.13370600 & -1.05123900 \\
\hline $\mathrm{C}$ & -5.58082800 & -5.03438300 & 0.79597600 \\
\hline $\mathrm{C}$ & -4.67239500 & -6.95090300 & -0.40500700 \\
\hline $\mathrm{C}$ & -4.50425800 & -5.90081000 & 0.51801700 \\
\hline $\mathrm{C}$ & 0.52228600 & -3.70373200 & -4.55522800 \\
\hline $\mathrm{O}$ & 0.33799700 & -4.00010900 & -5.95242800 \\
\hline $\mathrm{C}$ & 0.06701800 & -2.30603600 & -4.25797900 \\
\hline $\mathrm{S}$ & -0.31680800 & -1.95532600 & -2.57966300 \\
\hline $\mathrm{C}$ & -0.13082100 & -1.36705000 & -5.22057400 \\
\hline $\mathrm{S}$ & -0.65715500 & 0.26714300 & -4.76992400 \\
\hline $\mathrm{C}$ & 0.07313900 & -1.66967400 & -6.69488400 \\
\hline $\mathrm{C}$ & 0.88276000 & -2.96731700 & -6.84354200 \\
\hline $\mathrm{C}$ & -4.82290700 & 3.08714600 & -2.57461600 \\
\hline $\mathrm{O}$ & -6.19109600 & 2.56408200 & -2.50520300 \\
\hline $\mathrm{C}$ & -3.77983500 & 2.02378700 & -2.26315500 \\
\hline $\mathrm{S}$ & -2.13675300 & 2.28432600 & -2.90006100 \\
\hline $\mathrm{C}$ & -4.10728800 & 0.87772800 & -1.60952100 \\
\hline $\mathrm{S}$ & -2.82309900 & -0.33896200 & -1.31185700 \\
\hline $\mathrm{C}$ & -5.53217700 & 0.55344700 & -1.19383400 \\
\hline $\mathrm{C}$ & -6.42365400 & 1.81320200 & -1.27160500 \\
\hline W & -0.80859700 & 0.35020500 & -2.4167870 \\
\hline
\end{tabular}




\begin{tabular}{|c|c|c|c|}
\hline $\mathrm{O}$ & -2.06949900 & -3.11449600 & 0.13650500 \\
\hline $\mathrm{O}$ & 3.66998700 & -7.66630100 & -2.50889700 \\
\hline $\mathrm{O}$ & 3.15761200 & -6.56098600 & -0.06691300 \\
\hline 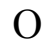 & 0.50919000 & -2.27241900 & 0.33253400 \\
\hline $\mathrm{O}$ & 0.25828900 & 0.22078100 & -0.51903800 \\
\hline 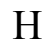 & -7.46361700 & -4.75339000 & -4.44463300 \\
\hline $\mathrm{H}$ & -6.02854100 & -7.92850000 & -1.78340600 \\
\hline $\mathrm{H}$ & -3.84554400 & -7.62331100 & -0.62428300 \\
\hline $\mathrm{H}$ & -3.55555200 & -5.76344700 & 1.03331000 \\
\hline $\mathrm{H}$ & -5.44034600 & -4.22409400 & 1.50844600 \\
\hline $\mathrm{H}$ & -7.65309700 & -4.56814800 & 0.38358800 \\
\hline $\mathrm{H}$ & -9.17298500 & -6.33864400 & -0.78224300 \\
\hline $\mathrm{H}$ & -8.40345500 & -7.49148200 & -1.88486100 \\
\hline $\mathrm{H}$ & -10.06301600 & -6.53014400 & -3.71871300 \\
\hline $\mathrm{H}$ & -10.30332700 & -4.83907600 & -3.72331700 \\
\hline $\mathrm{H}$ & -8.49897500 & -4.44818300 & -2.26104700 \\
\hline $\mathrm{H}$ & 1.94052400 & -2.79604600 & -6.60102700 \\
\hline $\mathrm{H}$ & 0.79326900 & -3.38624600 & -7.85126000 \\
\hline $\mathrm{H}$ & -0.90585400 & -1.77035900 & -7.18609800 \\
\hline $\mathrm{H}$ & 0.59935100 & -0.84072900 & -7.19007300 \\
\hline $\mathrm{H}$ & -0.07709500 & -4.44293900 & -4.01053000 \\
\hline $\mathrm{H}$ & 1.58098200 & -3.82837400 & -4.27362000 \\
\hline $\mathrm{H}$ & -4.72227200 & 3.93823000 & -1.88011400 \\
\hline $\mathrm{H}$ & -4.70468300 & 3.45466500 & -3.59862000 \\
\hline $\mathrm{H}$ & -5.55365800 & 0.15630300 & -0.16858200 \\
\hline $\mathrm{H}$ & -5.92964400 & -0.23177600 & -1.85514700 \\
\hline $\mathrm{H}$ & -6.23682400 & 2.46480400 & -0.40789600 \\
\hline $\mathrm{H}$ & -7.48552900 & 1.54441100 & -1.29514200 \\
\hline $\mathrm{H}$ & 0.13017200 & 9.22919100 & -1.13896400 \\
\hline $\mathrm{H}$ & 4.31994800 & 7.93644300 & -4.77310700 \\
\hline $\mathrm{H}$ & 2.97027500 & 5.84686100 & -5.02602700 \\
\hline $\mathrm{H}$ & 1.50909300 & 5.03638200 & -3.18037000 \\
\hline $\mathrm{H}$ & 1.32320100 & 6.30223500 & -1.05707700 \\
\hline $\mathrm{H}$ & 4.85919200 & 10.01005600 & -2.86568100 \\
\hline $\mathrm{H}$ & 4.14953200 & 10.63839000 & -0.50619000 \\
\hline $\mathrm{H}$ & 1.87697900 & 7.74293700 & 0.98416600 \\
\hline $\mathrm{H}$ & 2.88155600 & 9.07730200 & 1.54338800 \\
\hline $\mathrm{H}$ & 0.44970500 & 8.83162200 & 2.72741700 \\
\hline $\mathrm{H}$ & -0.35225600 & 10.26574600 & 2.43981100 \\
\hline $\mathrm{H}$ & 1.27436400 & 10.71998700 & 0.59743200 \\
\hline $\mathrm{H}$ & 7.34246300 & -1.45868000 & 9.66134300 \\
\hline $\mathrm{H}$ & 4.73475200 & -3.14312400 & 8.40643900 \\
\hline $\mathrm{H}$ & 4.46586500 & -1.92338700 & 9.53230300 \\
\hline $\mathrm{H}$ & 6.47256000 & -1.98093900 & 7.35463200 \\
\hline $\mathrm{H}$ & 4.19121100 & -0.01993800 & 7.90351500 \\
\hline $\mathrm{H}$ & 5.63256600 & 0.51936800 & 7.07259400 \\
\hline $\mathrm{H}$ & 5.93410700 & -2.33313500 & 5.12087000 \\
\hline $\mathrm{H}$ & 4.45266800 & -2.39925900 & 3.07886100 \\
\hline 11 & 1.91682500 & -1.28980800 & 2.23024900 \\
\hline 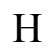 & 0.21231100 & 0.34895600 & 2.96423900 \\
\hline
\end{tabular}




\begin{tabular}{|c|c|c|c|}
\hline $\mathrm{H}$ & 0.37787400 & 1.48317500 & 5.17628100 \\
\hline $\mathrm{H}$ & 2.29498500 & 1.02528200 & 6.70192500 \\
\hline $\mathrm{H}$ & 2.46180900 & 0.07330000 & -3.01596300 \\
\hline $\mathrm{H}$ & 1.90329600 & 0.91246300 & -4.4789440 \\
\hline $\mathrm{H}$ & 5.35798400 & 1.37373000 & -4.91624000 \\
\hline $\mathrm{H}$ & 4.06766300 & 0.26913600 & -5.11501500 \\
\hline $\mathrm{H}$ & 3.55149100 & 2.75481800 & -4.0127930 \\
\hline $\mathrm{H}$ & 4.64619300 & 3.85791600 & -2.4494350 \\
\hline $\mathrm{H}$ & 5.67187400 & 4.47759100 & -0.53009200 \\
\hline $\mathrm{H}$ & 4.27240700 & 2.07070200 & 0.7400270 \\
\hline $\mathrm{H}$ & 6.11054800 & 3.05158400 & 2.20584500 \\
\hline $\mathrm{H}$ & 5.37490700 & 4.65132800 & 2.03249200 \\
\hline $\mathrm{H}$ & 4.50224400 & 3.35216900 & 2.87281000 \\
\hline $\mathrm{H}$ & 2.38447000 & 4.07935600 & 2.53670600 \\
\hline $\mathrm{H}$ & 1.13379500 & 4.08647200 & $1.2791300 \mathrm{C}$ \\
\hline $\mathrm{H}$ & 1.93898800 & 2.57235900 & 1.72205600 \\
\hline $\mathrm{H}$ & 3.36772300 & 4.98470000 & 0.40998200 \\
\hline $\mathrm{H}$ & 2.72945400 & 3.60297600 & -0.4889910 \\
\hline $\mathrm{H}$ & 6.86651500 & 1.67010300 & -0.77744100 \\
\hline $\mathrm{H}$ & -4.31226200 & 0.00941900 & 4.54884900 \\
\hline $\mathrm{H}$ & -5.56486400 & -2.73644700 & 3.8299680 \\
\hline $\mathrm{H}$ & -5.13679900 & -1.64856800 & 2.5940790 \\
\hline $\mathrm{H}$ & -3.26964800 & -2.89389300 & 4.5529970 \\
\hline $\mathrm{H}$ & -1.48216200 & -1.63260500 & 3.4734480 \\
\hline $\mathrm{H}$ & -2.34899700 & -2.55352000 & 2.2337080 \\
\hline $\mathrm{H}$ & -11.70283700 & 3.79995100 & 3.9766890 \\
\hline $\mathrm{H}$ & -9.97228200 & 2.15217100 & 1.5193720 \\
\hline $\mathrm{H}$ & -10.81414700 & 3.57043400 & 1.212226 \\
\hline $\mathrm{H}$ & -9.28817400 & 2.82854500 & 3.6452820 \\
\hline $\mathrm{H}$ & -8.92578200 & 5.16249200 & 1.71171400 \\
\hline $\mathrm{H}$ & -8.45260000 & 5.32493400 & 3.39265100 \\
\hline $\mathrm{H}$ & -7.36587000 & 1.77427200 & 2.77111600 \\
\hline $\mathrm{H}$ & -4.91962500 & 1.66835100 & 2.0559370 \\
\hline $\mathrm{H}$ & -2.91647900 & 3.46514000 & 1.11505800 \\
\hline $\mathrm{H}$ & -2.58632300 & 5.87572200 & 0.54059800 \\
\hline $\mathrm{H}$ & -4.43181500 & 7.50762100 & 0.77076400 \\
\hline $\mathrm{H}$ & -6.68962300 & 6.78680400 & 1.5640340 \\
\hline $\mathrm{H}$ & 6.56661800 & -1.50565800 & -4.7241500 \\
\hline $\mathrm{H}$ & 2.66127900 & -5.97275800 & -4.6048730 \\
\hline $\mathrm{H}$ & 2.62658300 & -5.12068400 & -6.1575100 \\
\hline $\mathrm{H}$ & 3.30569800 & -6.76452900 & -6.0557970 \\
\hline $\mathrm{H}$ & 4.78896900 & -6.66878300 & -3.6642820 \\
\hline $\mathrm{H}$ & 6.14671500 & -6.59865300 & -4.6841820 \\
\hline $\mathrm{H}$ & 5.17769000 & -5.19840200 & -6.1819500 \\
\hline $\mathrm{H}$ & 5.75655800 & -4.34190900 & -3.2442160 \\
\hline $\mathrm{H}$ & 4.47515000 & -1.61964700 & -3.3603630 \\
\hline $\mathrm{H}$ & 5.11148500 & -1.12544900 & -1.1769390 \\
\hline $\mathrm{H}$ & 4.41913000 & -2.74157000 & -1.1447020 \\
\hline $\mathrm{H}$ & 6.71784000 & -3.75586000 & -0.8736150 \\
\hline $\mathrm{H}$ & 7.44559500 & -2.14627300 & $-1.053937 \mathrm{C}$ \\
\hline
\end{tabular}




$\begin{array}{lrrr}\mathrm{H} & 6.48389800 & -0.41452300 & 2.35331700 \\ \mathrm{H} & 7.55335900 & -0.30621900 & 0.92436800 \\ \mathrm{H} & 5.77624100 & -0.16195500 & 0.73251000 \\ \mathrm{H} & -0.24050600 & -7.17543700 & 3.95658300 \\ \mathrm{H} & 2.73861600 & -7.59059300 & 4.71556500 \\ \mathrm{H} & 2.29363300 & -6.62696800 & 3.39760500 \\ \mathrm{H} & 1.56767600 & -6.19801100 & 6.26331500 \\ \mathrm{H} & 2.06738900 & -4.19005900 & 4.75729100 \\ \mathrm{H} & -0.78043600 & -4.62873400 & 3.65814000 \\ \mathrm{H} & 0.38450800 & -3.37051200 & 3.20703200 \\ \mathrm{H} & 0.67030800 & -5.04428000 & 2.72602900 \\ \mathrm{H} & 1.54910700 & -4.57715600 & 7.72558100 \\ \mathrm{H} & 2.11427100 & -3.04675100 & 7.00932900 \\ \mathrm{H} & 0.66506300 & -3.07375800 & 8.03461400 \\ \mathrm{H} & 0.02391300 & -2.87860000 & 5.64462600 \\ \mathrm{H} & -0.57322600 & -4.40023100 & 6.30514800 \\ \mathrm{H} & -2.25715300 & -3.94965400 & -0.33373400 \\ \mathrm{H} & -2.54855500 & -2.36911700 & -0.31296100 \\ \mathrm{H} & -0.43030800 & -2.60314000 & 0.45672100 \\ \mathrm{H} & 0.75698700 & -2.51093900 & -0.59395300 \\ \mathrm{H} & 3.12005300 & -4.98083900 & 2.01303200 \\ \mathrm{H} & -1.70823900 & 0.47225300 & 0.69386700 \\ \mathrm{H} & 2.99404400 & -8.28611800 & -2.83717800 \\ \mathrm{H} & 3.48140800 & -7.38253500 & -1.57470500 \\ \mathrm{H} & 2.22009800 & -3.76137400 & 1.03496000 \\ \mathrm{H} & 0.38400500 & -0.64035900 & -0.00162600 \\ \mathrm{H} & 1.02600500 & 0.85076500 & -0.55172900 \\ \mathrm{C} & 3.10816200 & -4.41161400 & 1.08035800 \\ \mathrm{C} & 3.14860800 & -5.30611700 & -0.11541600 \\ \mathrm{H} & 3.98224400 & -3.74109100 & 1.05091700 \\ \mathrm{H} & 3.18151000 & -4.80469200 & -1.10081700\end{array}$

\begin{tabular}{lrrr}
\multicolumn{5}{l}{$\mathbf{X N - E}, E=-5352.338891$} \\
$\mathrm{~N}$ & 5.36744200 & 2.62888100 & 0.28275300 \\
$\mathrm{C}$ & 4.32898300 & 3.55579600 & 0.78596400 \\
$\mathrm{C}$ & 4.06839400 & 3.47223900 & 2.31713300 \\
$\mathrm{O}$ & 3.13901100 & 4.09053900 & 2.86770500 \\
$\mathrm{C}$ & 2.98869300 & 3.53178100 & -0.00897100 \\
$\mathrm{C}$ & 2.56899900 & 2.17220600 & -0.55891000 \\
$\mathrm{O}$ & 2.91495000 & 1.10764200 & 0.19626600 \\
$\mathrm{O}$ & 1.96940100 & 2.09876300 & -1.64765300 \\
$\mathrm{~N}$ & -0.58282000 & 7.23533000 & -3.64721000 \\
$\mathrm{C}$ & 0.45379700 & 7.88792700 & -2.83813000 \\
$\mathrm{C}$ & 1.86076800 & 7.38685000 & -3.21172600 \\
$\mathrm{O}$ & 2.81735500 & 8.12554400 & -3.53407300 \\
$\mathrm{C}$ & 0.15899200 & 7.72220500 & -1.31447900 \\
$\mathrm{C}$ & 1.08195800 & 8.59786800 & -0.42553900 \\
$\mathrm{C}$ & 0.20571400 & 6.24548000 & -0.85069300 \\
$\mathrm{C}$ & 0.95509100 & 10.12068700 & -0.65228300 \\
$\mathrm{~N}$ & -3.03363500 & -0.06399900 & -7.90558600 \\
& & & $\mathrm{~S} 60$
\end{tabular}




\begin{tabular}{|c|c|c|c|}
\hline $\mathrm{C}$ & -4.02711000 & -1.14612000 & -7.87389500 \\
\hline $\mathrm{C}$ & -4.81077800 & -1.33352100 & -6.54979900 \\
\hline $\mathrm{O}$ & -5.74754500 & -2.19315700 & -6.47949900 \\
\hline C & -3.40170400 & -2.47022000 & -8.35730400 \\
\hline $\mathrm{N}$ & -4.51985400 & -0.49561700 & -5.51515500 \\
\hline $\mathrm{C}$ & -5.17620100 & -0.66314900 & -4.20276900 \\
\hline $\mathrm{C}$ & -6.65630500 & -0.99301600 & -4.36855400 \\
\hline $\mathrm{O}$ & -7.28806800 & -1.71369000 & -3.56821000 \\
\hline $\mathrm{C}$ & -4.98521700 & 0.62423000 & -3.33770900 \\
\hline $\mathrm{C}$ & -6.00828200 & 1.74748300 & -3.58125900 \\
\hline $\mathrm{S}$ & -5.53050000 & 3.35357600 & -2.77143800 \\
\hline $\mathrm{C}$ & -5.57607600 & 2.85143600 & -0.99178000 \\
\hline $\mathrm{N}$ & -5.42902300 & -3.81241100 & -2.16482400 \\
\hline $\mathrm{C}$ & -4.49881100 & -3.48693100 & -1.06123200 \\
\hline $\mathrm{C}$ & -5.06332600 & -2.23146100 & -0.33979500 \\
\hline $\mathrm{O}$ & -5.00607500 & -1.08590300 & -0.86794100 \\
\hline $\mathrm{C}$ & -3.04475700 & -3.25637600 & -1.48143100 \\
\hline $\mathrm{S}$ & -1.96438300 & -3.35485000 & 0.02136400 \\
\hline $\mathrm{N}$ & -5.67687000 & -2.40258600 & 0.88013000 \\
\hline $\mathrm{C}$ & -6.18464300 & -1.28883600 & 1.72602400 \\
\hline $\mathrm{C}$ & -7.38496300 & -0.58325900 & 1.06648100 \\
\hline $\mathrm{O}$ & -8.37760600 & -0.18600700 & 1.70830500 \\
\hline $\mathrm{C}$ & -5.07504900 & -0.24208100 & 2.12814300 \\
\hline $\mathrm{C}$ & -3.84951700 & -0.97096300 & 2.73982000 \\
\hline $\mathrm{C}$ & -5.65610400 & 0.80798500 & 3.10400200 \\
\hline $\mathrm{C}$ & -2.64523100 & -0.04277500 & 3.00743000 \\
\hline $\mathrm{N}$ & 9.55691800 & -2.27201400 & 4.78498900 \\
\hline $\mathrm{C}$ & 8.95039700 & -1.28420700 & 5.71115900 \\
\hline $\mathrm{C}$ & 9.70181500 & -1.34348100 & 7.04318000 \\
\hline $\mathrm{O}$ & 9.23672700 & -1.02234400 & 8.15184200 \\
\hline $\mathrm{C}$ & 7.42066400 & -1.36711700 & 5.92906200 \\
\hline $\mathrm{C}$ & 6.57121900 & -0.83341000 & 4.80317700 \\
\hline $\mathrm{C}$ & 6.91685500 & -0.00089100 & 3.74758100 \\
\hline $\mathrm{C}$ & 5.14508300 & -1.07352500 & 4.68833300 \\
\hline $\mathrm{N}$ & 5.77997600 & 0.29363400 & 2.98184300 \\
\hline $\mathrm{C}$ & 4.67607600 & -0.36029100 & 3.54173700 \\
\hline $\mathrm{C}$ & 4.23164300 & -1.84331000 & 5.44921400 \\
\hline $\mathrm{C}$ & 3.32868100 & -0.40224400 & 3.13363900 \\
\hline $\mathrm{C}$ & 2.89203200 & -1.89527600 & 5.04240700 \\
\hline $\mathrm{C}$ & 2.44866700 & -1.18483700 & 3.89247900 \\
\hline $\mathrm{N}$ & -2.55310700 & -3.30579300 & 9.35648900 \\
\hline $\mathrm{C}$ & -3.11094200 & -4.25762200 & 8.38357400 \\
\hline $\mathrm{C}$ & -2.04158600 & -5.11398300 & 7.68904600 \\
\hline $\mathrm{O}$ & -0.85530300 & -5.15550600 & 8.07063900 \\
\hline $\mathrm{C}$ & -4.02618700 & -3.51566300 & 7.36086300 \\
\hline $\mathrm{C}$ & -4.89393500 & -4.41365000 & 6.51212800 \\
\hline $\mathrm{C}$ & -6.10327500 & -4.97718900 & 6.89223300 \\
\hline $\mathrm{C}$ & -4.64946800 & -4.84039400 & 5.14286400 \\
\hline $\mathrm{N}$ & -6.62472800 & -5.73629100 & 5.83889400 \\
\hline $\mathrm{C}$ & -5.75255700 & -5.66267300 & 4.74801600 \\
\hline
\end{tabular}




$\begin{array}{lccc}\mathrm{C} & -3.61553800 & -4.58508000 & 4.20749800 \\ \mathrm{C} & -5.84874300 & -6.22821200 & 3.46179800 \\ \mathrm{C} & -3.70143900 & -5.14082600 & 2.92391400 \\ \mathrm{C} & -4.81026400 & -5.95507800 & 2.55721900 \\ \mathrm{~N} & -3.73745500 & 9.38035400 & 2.00221800 \\ \mathrm{C} & -4.37417900 & 8.36502400 & 2.85412000 \\ \mathrm{C} & -5.72552900 & 8.98697300 & 3.18422500 \\ \mathrm{O} & -6.15751600 & 9.21588700 & 4.33071900 \\ \mathrm{C} & -3.59632900 & 7.90259800 & 4.11682800 \\ \mathrm{C} & -2.28171200 & 7.21715200 & 3.82905100 \\ \mathrm{C} & -2.09264500 & 5.87727300 & 3.51368200 \\ \mathrm{C} & -0.95808400 & 7.81505200 & 3.87408200 \\ \mathrm{~N} & -0.73015300 & 5.61127600 & 3.35586300 \\ \mathrm{C} & -0.00856000 & 6.78241400 & 3.57904800 \\ \mathrm{C} & -0.48002800 & 9.11905300 & 4.15423100 \\ \mathrm{C} & 1.38164000 & 7.00705300 & 3.56226400 \\ \mathrm{C} & 0.90166900 & 9.35466400 & 4.13509600 \\ \mathrm{C} & 1.82213500 & 8.30870300 & 3.84251300 \\ \mathrm{~N} & 10.35394000 & -2.19594200 & -5.22280300 \\ \mathrm{C} & 9.04001500 & -1.83930600 & -4.73413400 \\ \mathrm{C} & 8.05745700 & -2.18962300 & -5.82643500 \\ \mathrm{O} & 8.38647000 & -2.79914800 & -6.86559400 \\ \mathrm{O} & -2.0436700 & -0.32974500 & -4.31146200 \\ \mathrm{O} & -1.54385200 & 4.54541100 & -3.55563100 \\ \mathrm{C} & -0.36403000 & 2.71339300 & -5.12010300 \\ \mathrm{C} & & & -4.13711300 \\ \mathrm{C} & & & \end{array}$




\begin{tabular}{|c|c|c|c|}
\hline $\mathrm{O}$ & -0.25594600 & -1.04004300 & -0.02235800 \\
\hline $\mathrm{H}$ & 7.00975500 & -1.90033500 & -5.65681400 \\
\hline $\mathrm{H}$ & 7.67602200 & 1.65277300 & -5.70759300 \\
\hline $\mathrm{H}$ & 5.29024400 & 2.36008200 & -5.58375000 \\
\hline & 3.72499100 & 1.22112600 & -4.00291200 \\
\hline $\mathrm{H}$ & 4.58760200 & -0.55089200 & -2.46756800 \\
\hline $\mathrm{H}$ & 6.98198800 & -1.20422800 & -2.53651800 \\
\hline$H$ & 9.51303300 & -0.21814300 & -3.38192100 \\
\hline & 9.45363000 & 0.24976100 & -5.08379900 \\
\hline $\mathrm{H}$ & 10.35464000 & -2.77422200 & -6.05725300 \\
\hline $\mathrm{H}$ & 11.07251100 & -2.39539700 & -4.53971100 \\
\hline IT & 8.71265900 & -2.44158100 & -3.86072100 \\
\hline & -2.02903200 & -4.26927400 & -6.36753100 \\
\hline $\mathrm{H}$ & -0.73293800 & -5.11960800 & -7.26342400 \\
\hline $\mathrm{H}$ & 0.46303000 & -5.63626700 & -5.21588000 \\
\hline $\mathrm{H}$ & -1.22827300 & -5.86869900 & -4.75886700 \\
\hline 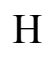 & 0.11655500 & -1.36525500 & -5.91773700 \\
\hline $\mathrm{H}$ & -1.59152500 & -1.87012200 & -5.85320800 \\
\hline $\mathrm{H}$ & 3.32263100 & -4.81876600 & 2.76024900 \\
\hline $\mathrm{H}$ & 3.27303200 & -5.99432000 & 1.41830000 \\
\hline $\mathrm{H}$ & 5.24949200 & -1.67780600 & 0.59176600 \\
\hline $\mathrm{H}$ & 5.50253400 & -3.02297800 & -0.53478500 \\
\hline $\mathrm{H}$ & 5.26704700 & -3.11122900 & 2.53278500 \\
\hline $\mathrm{H}$ & 6.63570300 & -3.67736600 & 1.52546300 \\
\hline $\mathrm{H}$ & -2.38752100 & -5.73834700 & 6.84519600 \\
\hline $\mathrm{H}$ & -6.69435300 & -6.84836800 & 3.17182400 \\
\hline $\mathrm{H}$ & -4.84551200 & -6.37299100 & 1.55377300 \\
\hline $\mathrm{H}$ & -2.93093900 & -4.93104400 & 2.18524300 \\
\hline $\mathrm{H}$ & -2.76659200 & -3.95552500 & 4.46434800 \\
\hline $\mathrm{H}$ & -7.50684900 & -6.22667800 & 5.85000900 \\
\hline $\mathrm{H}$ & -6.63631200 & -4.88229700 & 7.82814500 \\
\hline $\mathrm{H}$ & -3.40054800 & -2.88049300 & 6.71730200 \\
\hline $\mathrm{H}$ & -4.65150900 & -2.84024700 & 7.95907300 \\
\hline $\mathrm{H}$ & -2.30356600 & -2.41247300 & 8.93221600 \\
\hline $\mathrm{H}$ & -1.75116400 & -3.69080900 & 9.85626200 \\
\hline $\mathrm{H}$ & -3.75501300 & -4.97896100 & 8.91602500 \\
\hline $\mathrm{H}$ & -6.29676100 & 9.28812400 & 2.28483000 \\
\hline $\mathrm{H}$ & -3.09002800 & 9.02145500 & 1.30671200 \\
\hline $\mathrm{H}$ & -3.35500000 & 10.17571700 & 2.50761700 \\
\hline $\mathrm{H}$ & -4.57171300 & 7.49247200 & 2.21315500 \\
\hline $\mathrm{H}$ & -3.43277600 & 8.77759700 & 4.76062100 \\
\hline $\mathrm{H}$ & -4.25897700 & 7.23667200 & 4.68623500 \\
\hline $\mathrm{H}$ & -2.82556100 & 5.09030100 & 3.40289100 \\
\hline $\mathrm{H}$ & -0.32449100 & 4.70961100 & 3.14344900 \\
\hline $\mathrm{H}$ & 2.07880300 & 6.20081900 & 3.34966500 \\
\hline $\mathrm{H}$ & 2.88865900 & 8.52347500 & 3.83950300 \\
\hline $\mathrm{H}$ & 1.28324200 & 10.35095700 & 4.34974100 \\
\hline $\mathrm{H}$ & -1.17123900 & 9.92551200 & 4.39209300 \\
\hline 11 & -2.92938300 & -2.28890200 & -1.98121100 \\
\hline 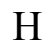 & -2.73503 & -4.04790200 & -2.17441 \\
\hline
\end{tabular}




\begin{tabular}{|c|c|c|c|}
\hline $\mathrm{H}$ & -6.02646300 & -3.06607900 & -2.51939400 \\
\hline $\mathrm{H}$ & -5.02037700 & -4.38645800 & -2.89617800 \\
\hline $\mathrm{H}$ & -4.49946400 & -4.32231400 & -0.34173100 \\
\hline $\mathrm{H}$ & -5.65224800 & -3.32830900 & 1.29464100 \\
\hline $\mathrm{H}$ & -6.55146400 & -1.74479400 & 2.65631500 \\
\hline $\mathrm{H}$ & -4.74642800 & 0.25877800 & 1.20832300 \\
\hline $\mathrm{H}$ & -6.51366200 & 1.34501500 & 2.68404100 \\
\hline $\mathrm{H}$ & -5.98943800 & 0.33156600 & 4.03780400 \\
\hline $\mathrm{H}$ & -4.89017200 & 1.54932400 & 3.36023400 \\
\hline $\mathrm{H}$ & -2.85552300 & 0.70742800 & 3.78196500 \\
\hline $\mathrm{H}$ & -1.77723700 & -0.62956200 & 3.33413800 \\
\hline $\mathrm{H}$ & -2.34918500 & 0.48375500 & 2.08976900 \\
\hline $\mathrm{H}$ & -4.15856500 & -1.46216400 & 3.67838900 \\
\hline $\mathrm{H}$ & -3.51559100 & -1.76096100 & 2.05716400 \\
\hline $\mathrm{H}$ & -7.30656100 & -0.43728700 & -0.02357000 \\
\hline $\mathrm{H}$ & 4.79108900 & 2.87001300 & 2.89581900 \\
\hline $\mathrm{H}$ & 6.03082600 & 3.03741300 & -0.36657800 \\
\hline $\mathrm{H}$ & 5.00503600 & 1.72873000 & -0.02160200 \\
\hline $\mathrm{H}$ & 4.73035400 & 4.57654700 & 0.66748400 \\
\hline $\mathrm{H}$ & 2.18158600 & 3.90994900 & 0.63229800 \\
\hline $\mathrm{H}$ & 3.04636700 & 4.19485700 & -0.87722600 \\
\hline $\mathrm{H}$ & 10.74730000 & -1.69853000 & 6.93656300 \\
\hline $\mathrm{H}$ & 9.37761100 & -2.06088300 & 3.80559000 \\
\hline $\mathrm{H}$ & 9.28513800 & -3.23227800 & 4.99588500 \\
\hline $\mathrm{H}$ & 9.19505400 & -0.28654300 & 5.30690100 \\
\hline $\mathrm{H}$ & 7.15781600 & -2.42012500 & 6.11953300 \\
\hline $\mathrm{H}$ & 7.18403800 & -0.82994900 & 6.85923800 \\
\hline $\mathrm{H}$ & 7.87689900 & 0.41640800 & 3.47608400 \\
\hline $\mathrm{H}$ & 5.77073400 & 0.87318200 & 2.14830300 \\
\hline $\mathrm{H}$ & 2.98089700 & 0.11977400 & 2.25003000 \\
\hline $\mathrm{H}$ & 1.41393300 & -1.27024900 & 3.57206800 \\
\hline $\mathrm{H}$ & 2.17921300 & -2.49895500 & 5.59954900 \\
\hline $\mathrm{H}$ & 4.56304000 & -2.39468300 & 6.32687800 \\
\hline $\mathrm{H}$ & -7.17917700 & -0.46603800 & -5.18664600 \\
\hline $\mathrm{H}$ & -2.47776200 & -2.69224800 & -7.81522300 \\
\hline $\mathrm{H}$ & -4.10991500 & -3.29403800 & -8.22027900 \\
\hline $\mathrm{H}$ & -3.14426300 & -2.37968600 & -9.41883100 \\
\hline $\mathrm{H}$ & -2.21276400 & -0.22562500 & -7.32487900 \\
\hline $\mathrm{H}$ & -3.41790100 & 0.86483300 & -7.74790900 \\
\hline $\mathrm{H}$ & -4.81698600 & -0.88549500 & -8.59599700 \\
\hline $\mathrm{H}$ & -3.63629900 & 0.04271400 & -5.49818900 \\
\hline $\mathrm{H}$ & -4.71325100 & -1.49171800 & -3.65098800 \\
\hline $\mathrm{H}$ & -5.02454400 & 0.30194300 & -2.29928100 \\
\hline $\mathrm{H}$ & -3.97648000 & 1.00400900 & -3.53401500 \\
\hline $\mathrm{H}$ & -6.08496600 & 2.00332500 & -4.64788100 \\
\hline $\mathrm{H}$ & -7.01010700 & 1.47454000 & -3.22474100 \\
\hline $\mathrm{H}$ & -5.33938800 & 3.74612100 & -0.40458500 \\
\hline $\mathrm{H}$ & -6.57675900 & 2.49550500 & -0.72093200 \\
\hline $\mathrm{H}$ & -4.83777500 & 2.07271700 & -0.78270600 \\
\hline $\mathrm{H}$ & 1.96194600 & 6.28197200 & -3.25363700 \\
\hline
\end{tabular}




$\begin{array}{lrrr}\mathrm{H} & -0.58573600 & 7.48164200 & -4.63142400 \\ \mathrm{H} & -0.87682500 & 6.26576700 & -3.46243700 \\ \mathrm{H} & 0.44535400 & 8.96289700 & -3.06406100 \\ \mathrm{H} & -0.87666600 & 8.08111300 & -1.19922200 \\ \mathrm{H} & 1.23502400 & 5.86170400 & -0.85947700 \\ \mathrm{H} & -0.16191100 & 6.16814600 & 0.18167500 \\ \mathrm{H} & -0.40424600 & 5.58769200 & -1.48003100 \\ \mathrm{H} & 1.33532200 & 10.41867500 & -1.63716300 \\ \mathrm{H} & -0.09411300 & 10.44266800 & -0.58073100 \\ \mathrm{H} & 1.52762200 & 10.67234100 & 0.10592600 \\ \mathrm{H} & 0.84752100 & 8.37455000 & 0.62536000 \\ \mathrm{H} & 2.13084800 & 8.29766200 & -0.57501200 \\ \mathrm{H} & 1.54318100 & 1.58039300 & -3.09020800 \\ \mathrm{H} & 1.06764200 & 0.29778100 & -3.94837600 \\ \mathrm{H} & -0.11851600 & 3.05961000 & -5.99692100 \\ \mathrm{H} & 0.40692600 & 2.12530700 & -4.67745600 \\ \mathrm{H} & -1.08292600 & 3.84877500 & -4.10979200 \\ \mathrm{H} & -2.43639100 & 4.25079700 & -3.28957900 \\ \mathrm{H} & -1.51335300 & 1.47731000 & -5.04998000 \\ \mathrm{H} & -1.62940900 & 0.07033300 & -4.23504600 \\ \mathrm{H} & 2.66925000 & 0.14782200 & -0.15622300 \\ \mathrm{H} & -1.14737200 & -1.09940900 & 0.39352800\end{array}$

$\begin{array}{lrrr}\text { XN-ES }, E=-5429.675630 & \\ \mathrm{~N} & 5.57568700 & 1.88104800 & 0.55635500 \\ \mathrm{C} & 5.04845400 & 3.02149200 & 1.34389600 \\ \mathrm{C} & 4.34353800 & 2.61854900 & 2.67194400 \\ \mathrm{O} & 3.97496500 & 3.48503600 & 3.48383000 \\ \mathrm{C} & 4.11165500 & 4.00769800 & 0.54897800 \\ \mathrm{C} & 2.91048500 & 3.29947900 & -0.04331200 \\ \mathrm{O} & 1.81718300 & 3.30803000 & 0.76423200 \\ \mathrm{O} & 2.93963200 & 2.73217100 & -1.15891900 \\ \mathrm{~N} & 0.19310500 & 7.50624000 & -2.82821400 \\ \mathrm{C} & 1.31248900 & 8.08648700 & -2.08251400 \\ \mathrm{C} & 2.63291500 & 7.34920300 & -2.37386700 \\ \mathrm{O} & 3.69526400 & 7.90772600 & -2.72229900 \\ \mathrm{C} & 1.00050100 & 8.14714100 & -0.55402800 \\ \mathrm{C} & 2.08297800 & 8.91180700 & 0.25376500 \\ \mathrm{C} & 0.75731400 & 6.74942400 & 0.06138700 \\ \mathrm{C} & 2.28409500 & 10.38734400 & -0.15653900 \\ \mathrm{~N} & -2.95760400 & 1.05352500 & -7.91270000 \\ \mathrm{C} & -4.04743900 & 0.07130600 & -7.99699600 \\ \mathrm{C} & -4.88119600 & -0.14330200 & -6.70991400 \\ \mathrm{O} & -5.93373900 & -0.85878000 & -6.74636600 \\ \mathrm{C} & -3.51357800 & -1.27208800 & -8.53638400 \\ \mathrm{~N} & -4.48660000 & 0.50169100 & -5.57580600 \\ \mathrm{C} & -5.17318500 & 0.25973400 & -4.28913300 \\ \mathrm{C} & -6.67956600 & 0.10876600 & -4.49012800 \\ \mathrm{O} & -7.38752600 & -0.65156000 & -3.79771100 \\ \mathrm{C} & -4.85751100 & 1.41914600 & -3.29094000\end{array}$




\begin{tabular}{|c|c|c|c|}
\hline $\mathrm{C}$ & -5.77535900 & 2.64796400 & -3.40354100 \\
\hline $\mathrm{S}$ & -5.12830300 & 4.13607400 & -2.48560700 \\
\hline $\mathrm{C}$ & -5.02832900 & 3.45643300 & -0.76741200 \\
\hline $\mathrm{N}$ & -5.69494400 & -3.03947600 & -2.70878200 \\
\hline $\mathrm{C}$ & -4.85492100 & -2.96951500 & -1.49963500 \\
\hline $\mathrm{C}$ & -5.32302400 & -1.75234300 & -0.6547930 \\
\hline $\mathrm{O}$ & -5.18002800 & -0.56811300 & -1.0691290 \\
\hline $\mathrm{C}$ & -3.34523300 & -2.86259100 & -1.79117100 \\
\hline $\mathrm{S}$ & -2.34880100 & -3.24644000 & -0.26849100 \\
\hline $\mathrm{N}$ & -5.93731200 & -1.99787900 & 0.5518030 \\
\hline $\mathrm{C}$ & -6.34307700 & -0.94033200 & 1.5184160 \\
\hline $\mathrm{C}$ & -7.45177800 & -0.04050600 & 0.9428190 \\
\hline $\mathrm{O}$ & -8.44310500 & 0.32160300 & 1.6071470 \\
\hline $\mathrm{C}$ & -5.13480100 & -0.07503900 & 2.0427350 \\
\hline $\mathrm{C}$ & -4.05830700 & -1.00164100 & 2.6672750 \\
\hline $\mathrm{C}$ & -5.62694300 & 0.98829700 & 3.0524960 \\
\hline $\mathrm{C}$ & -2.76617400 & -0.26556800 & 3.0825040 \\
\hline $\mathrm{N}$ & 9.16856800 & -3.91513800 & 4.4724250 \\
\hline $\mathrm{C}$ & 8.75025300 & -2.90131100 & 5.47303100 \\
\hline $\mathrm{C}$ & 9.37820000 & -3.27097400 & $6.8226870 \mathrm{C}$ \\
\hline $\mathrm{O}$ & 8.94977200 & -2.92377200 & 7.93819500 \\
\hline $\mathrm{C}$ & 7.22981200 & -2.63705000 & 5.62950900 \\
\hline $\mathrm{C}$ & 6.54859100 & -1.91617800 & 4.49010200 \\
\hline $\mathrm{C}$ & 7.06726900 & -1.04156200 & 3.54240200 \\
\hline $\mathrm{C}$ & 5.11984300 & -1.98883500 & 4.24131800 \\
\hline $\mathrm{N}$ & 6.03975100 & -0.57187400 & 2.71090300 \\
\hline $\mathrm{C}$ & 4.83381300 & -1.14976800 & 3.11963800 \\
\hline $\mathrm{C}$ & 4.06420500 & -2.70480200 & 4.8573050 \\
\hline $\mathrm{C}$ & 3.53613700 & -1.03995900 & $2.5800270 \mathrm{C}$ \\
\hline $\mathrm{C}$ & 2.77038100 & -2.58667000 & 4.33184600 \\
\hline $\mathrm{C}$ & 2.51222400 & -1.77277100 & 3.19449300 \\
\hline $\mathrm{N}$ & -3.03699800 & -4.14274200 & 8.8364650 \\
\hline $\mathrm{C}$ & -3.68589100 & -4.95539900 & 7.7962830 \\
\hline $\mathrm{C}$ & -2.70213800 & -5.83452300 & 7.00976300 \\
\hline $\mathrm{O}$ & -1.52509900 & -6.02437700 & 7.3744000 \\
\hline $\mathrm{C}$ & -4.54804300 & -4.05495900 & 6.8587060 \\
\hline $\mathrm{C}$ & -5.49246700 & -4.80545000 & 5.9501130 \\
\hline $\mathrm{C}$ & -6.71946200 & -5.34319800 & 6.3101910 \\
\hline $\mathrm{C}$ & -5.31284900 & -5.09280100 & 4.5351630 \\
\hline $\mathrm{N}$ & -7.31336900 & -5.95490000 & 5.20038100 \\
\hline $\mathrm{C}$ & -6.47068300 & -5.80949600 & 4.0937820 \\
\hline $\mathrm{C}$ & -4.29361500 & -4.79312400 & 3.5972530 \\
\hline $\mathrm{C}$ & -6.63418400 & -6.22932100 & 2.7595690 \\
\hline $\mathrm{C}$ & -4.44557000 & -5.20535200 & 2.26613600 \\
\hline $\mathrm{C}$ & -5.60770200 & -5.91702400 & 1.8540340( \\
\hline $\mathrm{N}$ & -2.79861100 & 9.31185700 & 3.0248400 \\
\hline $\mathrm{C}$ & -3.60692300 & 8.24540700 & 3.6383420 \\
\hline $\mathrm{C}$ & -4.83303000 & 8.99734000 & 4.15022300 \\
\hline $\mathrm{O}$ & -5.19828400 & 9.06209800 & 5.33980900 \\
\hline $\mathrm{C}$ & -2.92504200 & 7.37642800 & 4.7310220 \\
\hline
\end{tabular}




\begin{tabular}{|c|c|c|c|}
\hline $\mathrm{C}$ & -1.63966100 & 6.70573300 & 4.30206800 \\
\hline $\mathrm{C}$ & -1.49372300 & 5.57098900 & 3.51174800 \\
\hline $\mathrm{C}$ & -0.29863900 & 7.09432300 & 4.70418500 \\
\hline $\mathrm{N}$ & -0.14215100 & 5.23422500 & 3.40346600 \\
\hline $\mathrm{C}$ & 0.61524000 & 6.14867300 & 4.13269500 \\
\hline $\mathrm{C}$ & 0.21510100 & 8.14024000 & 5.51023900 \\
\hline $\mathrm{C}$ & 2.00500100 & 6.20490800 & 4.35798300 \\
\hline $\mathrm{C}$ & 1.59821900 & 8.21468000 & 5.72527300 \\
\hline $\mathrm{C}$ & 2.48233300 & 7.25407200 & 5.15742400 \\
\hline $\mathrm{N}$ & 10.10767400 & -2.78092600 & -5.45876500 \\
\hline $\mathrm{C}$ & 8.82948200 & -2.35519600 & -4.93280100 \\
\hline $\mathrm{C}$ & 7.83294700 & -2.46424200 & -6.06173200 \\
\hline $\mathrm{O}$ & 8.10852500 & -2.97837400 & -7.16581200 \\
\hline $\mathrm{C}$ & 8.88243400 & -0.90788500 & -4.32927100 \\
\hline $\mathrm{C}$ & 7.52318600 & -0.29122600 & -4.02964900 \\
\hline $\mathrm{C}$ & 6.60749600 & -0.90412200 & -3.14164800 \\
\hline $\mathrm{C}$ & 7.12616900 & 0.89037800 & -4.69450000 \\
\hline $\mathrm{C}$ & 5.31808400 & -0.37149600 & -2.94755700 \\
\hline $\mathrm{C}$ & 5.84468200 & 1.43749600 & -4.49064500 \\
\hline $\mathrm{C}$ & 4.93184300 & 0.80263300 & -3.62718400 \\
\hline $\mathrm{C}$ & -0.36987200 & -1.57594800 & -5.90791600 \\
\hline $\mathrm{O}$ & -0.49181700 & -2.53372100 & -7.01277700 \\
\hline $\mathrm{C}$ & -0.29135600 & -2.23269400 & -4.54309800 \\
\hline $\mathrm{S}$ & 0.10256600 & -1.20367600 & -3.13631600 \\
\hline $\mathrm{C}$ & -0.46725000 & -3.57170300 & -4.36295200 \\
\hline $\mathrm{S}$ & -0.32243300 & -4.32552200 & -2.75886000 \\
\hline $\mathrm{C}$ & -0.77448400 & -4.49076700 & -5.53684300 \\
\hline $\mathrm{C}$ & -1.35048500 & -3.67876500 & -6.70283600 \\
\hline $\mathrm{C}$ & 3.01222500 & -5.56903700 & 1.23120900 \\
\hline $\mathrm{O}$ & 4.43662500 & -5.71939000 & 0.93473900 \\
\hline $\mathrm{C}$ & 2.40392000 & -4.38057400 & 0.51898100 \\
\hline $\mathrm{S}$ & 0.64485100 & -4.47720800 & 0.22302500 \\
\hline $\mathrm{C}$ & 3.15621100 & -3.36863900 & 0.01632500 \\
\hline $\mathrm{S}$ & 2.38716400 & -1.99016900 & -0.84782600 \\
\hline $\mathrm{C}$ & 4.67517500 & -3.39736200 & 0.09138500 \\
\hline $\mathrm{C}$ & 5.15480900 & -4.45576000 & 1.10366400 \\
\hline W & 0.07051800 & -2.61296000 & -1.10917500 \\
\hline $\mathrm{O}$ & 1.51694500 & 1.70169100 & -3.26822300 \\
\hline $\mathrm{O}$ & -1.85979700 & 1.25347900 & -4.77446700 \\
\hline $\mathrm{O}$ & -1.34610800 & 4.99820100 & -2.71198000 \\
\hline $\mathrm{O}$ & -0.09094700 & 3.27959500 & -4.32020400 \\
\hline $\mathrm{O}$ & -0.53024900 & -1.03101900 & 0.00184500 \\
\hline $\mathrm{H}$ & 6.82104300 & -2.08080300 & -5.85973800 \\
\hline $\mathrm{H}$ & 7.81728400 & 1.37231500 & -5.38486900 \\
\hline $\mathrm{H}$ & 5.55176500 & 2.34378200 & -5.01678800 \\
\hline $\mathrm{H}$ & 3.92900000 & 1.19976900 & -3.50435300 \\
\hline $\mathrm{H}$ & 4.60365800 & -0.87919200 & -2.30073600 \\
\hline $\mathrm{H}$ & 6.88874400 & -1.81322800 & -2.61101900 \\
\hline $\mathrm{H}$ & 9.49643200 & -0.96995100 & -3.41832500 \\
\hline $\mathrm{H}$ & 9.43511400 & -0.28815500 & -5.04460500 \\
\hline
\end{tabular}




\begin{tabular}{|c|c|c|c|}
\hline $\mathrm{H}$ & 10.06166000 & -3.25898800 & -6.35327200 \\
\hline $\mathrm{H}$ & 10.79335500 & -3.12950300 & -4.80197000 \\
\hline $\mathrm{H}$ & 8.42956400 & -3.02217000 & -4.14033700 \\
\hline $\mathrm{H}$ & -2.36644500 & -3.33167200 & -6.44925600 \\
\hline $\mathrm{H}$ & -1.39182100 & -4.25981200 & -7.63128100 \\
\hline $\mathrm{H}$ & 0.14250600 & -5.00761900 & -5.85804200 \\
\hline $\mathrm{H}$ & -1.49201900 & -5.26618000 & -5.23114500 \\
\hline $\mathrm{H}$ & 0.54423700 & -1.00944900 & -6.12473600 \\
\hline $\mathrm{H}$ & -1.21963100 & -0.87484700 & -5.95127600 \\
\hline $\mathrm{H}$ & 2.87795800 & -5.48235500 & 2.32574500 \\
\hline $\mathrm{H}$ & 2.54563700 & -6.50184300 & 0.89713300 \\
\hline $\mathrm{H}$ & 5.07166100 & -2.41447900 & 0.38659000 \\
\hline $\mathrm{H}$ & 5.07901800 & -3.62430200 & -0.90937800 \\
\hline $\mathrm{H}$ & 5.00825100 & -4.08452200 & 2.13089900 \\
\hline $\mathrm{H}$ & 6.21349800 & -4.70313800 & 0.95754500 \\
\hline $\mathrm{H}$ & -3.10542800 & -6.33703000 & 6.11167400 \\
\hline $\mathrm{H}$ & -7.52111700 & -6.76971800 & 2.43529400 \\
\hline $\mathrm{H}$ & -5.69499100 & -6.22435100 & 0.81440600 \\
\hline $\mathrm{H}$ & -3.67808400 & -4.96374500 & 1.53333900 \\
\hline $\mathrm{H}$ & -3.40285900 & -4.24169500 & 3.88925600 \\
\hline $\mathrm{H}$ & -8.21991600 & -6.39846000 & 5.19067200 \\
\hline $\mathrm{H}$ & -7.21858400 & -5.32388600 & 7.26915100 \\
\hline $\mathrm{H}$ & -3.88003600 & -3.41788500 & 6.26153800 \\
\hline $\mathrm{H}$ & -5.11059600 & -3.38649000 & 7.52344000 \\
\hline $\mathrm{H}$ & -2.72396300 & -3.23662100 & 8.48841400 \\
\hline $\mathrm{H}$ & -2.26105700 & -4.63404500 & 9.28107200 \\
\hline $\mathrm{H}$ & -4.38147500 & -5.66488500 & 8.27772000 \\
\hline $\mathrm{H}$ & -5.36253800 & 9.55905600 & 3.35634300 \\
\hline $\mathrm{H}$ & -2.20489400 & 9.00963400 & 2.25759200 \\
\hline $\mathrm{H}$ & -2.28891000 & 9.88449700 & 3.69410000 \\
\hline $\mathrm{H}$ & -3.94847800 & 7.59719300 & 2.81722300 \\
\hline $\mathrm{H}$ & -2.73569600 & 8.01292300 & 5.60542700 \\
\hline $\mathrm{H}$ & -3.65863300 & 6.62929600 & 5.06508800 \\
\hline $\mathrm{H}$ & -2.24992500 & 4.96520500 & 3.03217900 \\
\hline $\mathrm{H}$ & 0.23444600 & 4.46330800 & 2.86471300 \\
\hline $\mathrm{H}$ & 2.67865800 & 5.45225500 & 3.95680200 \\
\hline $\mathrm{H}$ & 3.54905300 & 7.32621300 & 5.35875500 \\
\hline $\mathrm{H}$ & 2.00727900 & 9.01033200 & 6.34508500 \\
\hline $\mathrm{H}$ & -0.45203500 & 8.86834000 & 5.96836800 \\
\hline $\mathrm{H}$ & -3.10108000 & -1.86432500 & -2.17115300 \\
\hline $\mathrm{H}$ & -3.07424900 & -3.60079800 & -2.55655100 \\
\hline $\mathrm{H}$ & -6.27917600 & -2.23736000 & -2.93959200 \\
\hline $\mathrm{H}$ & -5.25084900 & -3.47470600 & -3.51032700 \\
\hline $\mathrm{H}$ & -5.00454600 & -3.88004400 & -0.89431000 \\
\hline $\mathrm{H}$ & -5.97498400 & -2.95947700 & 0.87378200 \\
\hline $\mathrm{H}$ & -6.77382900 & -1.45882700 & 2.38633900 \\
\hline $\mathrm{H}$ & -4.68914600 & 0.42912500 & 1.17540400 \\
\hline $\mathrm{H}$ & -6.34925000 & 1.68532600 & 2.61221900 \\
\hline $\mathrm{H}$ & -6.11241900 & 0.51385300 & 3.91795400 \\
\hline $\mathrm{H}$ & -4.77998400 & 1.57753000 & 3.42201500 \\
\hline
\end{tabular}




\begin{tabular}{|c|c|c|c|}
\hline $\mathrm{H}$ & -2.92766100 & 0.42814800 & 3.91847600 \\
\hline $\mathrm{H}$ & -1.99717100 & -0.98655800 & 3.38815300 \\
\hline $\mathrm{H}$ & -2.35448800 & 0.30624200 & 2.24010900 \\
\hline H & -4.49340000 & -1.51337000 & 3.54327400 \\
\hline $\mathrm{H}$ & -3.78544600 & -1.77635400 & 1.94056200 \\
\hline $\mathrm{H}$ & -7.30688900 & 0.28146200 & -0.10130400 \\
\hline $\mathrm{H}$ & 4.19725300 & 1.54421400 & 2.86296700 \\
\hline $\mathrm{H}$ & 6.37345100 & 2.13103800 & -0.02438400 \\
\hline $\mathrm{H}$ & 4.87372700 & 1.39382800 & -0.00443700 \\
\hline $\mathrm{H}$ & 5.91151100 & 3.62628500 & 1.65691200 \\
\hline $\mathrm{H}$ & 3.77885300 & 4.79459900 & 1.23108300 \\
\hline $\mathrm{H}$ & 4.68406600 & 4.44318500 & -0.27596100 \\
\hline $\mathrm{H}$ & 10.29320700 & -3.88981600 & 6.72170100 \\
\hline $\mathrm{H}$ & 9.08591400 & -3.57979000 & 3.51455800 \\
\hline $\mathrm{H}$ & 8.67404700 & -4.80158800 & 4.57761800 \\
\hline H & 9.24410100 & -1.95700200 & 5.18481900 \\
\hline $\mathrm{H}$ & 6.73298800 & -3.60766800 & 5.78530700 \\
\hline $\mathrm{H}$ & 7.08672900 & -2.07970000 & 6.56698300 \\
\hline $\mathrm{H}$ & 8.08512100 & -0.70690300 & 3.39362800 \\
\hline $\mathrm{H}$ & 6.12799800 & 0.11104700 & 1.95692800 \\
\hline $\mathrm{H}$ & 3.32535900 & -0.48933500 & 1.66759900 \\
\hline $\mathrm{H}$ & 1.51800800 & -1.74935300 & 2.75760800 \\
\hline $\mathrm{H}$ & 1.95093600 & -3.14631000 & 4.77658300 \\
\hline $\mathrm{H}$ & 4.25206000 & -3.34631400 & 5.71611300 \\
\hline $\mathrm{H}$ & -7.14126600 & 0.80167200 & -5.21561700 \\
\hline $\mathrm{H}$ & -2.65890900 & -1.62338400 & -7.94843200 \\
\hline $\mathrm{H}$ & -4.30445700 & -2.02906400 & -8.51283100 \\
\hline $\mathrm{H}$ & -3.16783400 & -1.13459700 & -9.56730200 \\
\hline $\mathrm{H}$ & -2.15164800 & 0.75043400 & -7.36877500 \\
\hline $\mathrm{H}$ & -3.24965400 & 1.99386500 & -7.65762600 \\
\hline $\mathrm{H}$ & -4.78303800 & 0.44706000 & -8.72418900 \\
\hline $\mathrm{H}$ & -3.54420400 & 0.91518500 & -5.49701500 \\
\hline $\mathrm{H}$ & -4.80577100 & -0.66813400 & -3.83247800 \\
\hline $\mathrm{H}$ & -4.92784000 & 0.99189200 & -2.29279100 \\
\hline $\mathrm{H}$ & -3.81730000 & 1.71980700 & -3.45746300 \\
\hline $\mathrm{H}$ & -5.85971100 & 3.00081300 & -4.44156700 \\
\hline $\mathrm{H}$ & -6.78632600 & 2.43911300 & -3.03086400 \\
\hline $\mathrm{H}$ & -4.75641000 & 4.29243500 & -0.11289200 \\
\hline $\mathrm{H}$ & -6.00226400 & 3.05868700 & -0.46113400 \\
\hline $\mathrm{H}$ & -4.26714900 & 2.67553700 & -0.69470100 \\
\hline $\mathrm{H}$ & 2.56715200 & 6.24153700 & -2.32275500 \\
\hline $\mathrm{H}$ & 0.19831000 & 7.65706700 & -3.83006400 \\
\hline $\mathrm{H}$ & -0.27541800 & 6.64218700 & -2.54272800 \\
\hline $\mathrm{H}$ & 1.46108000 & 9.11792800 & -2.42981400 \\
\hline $\mathrm{H}$ & 0.05767700 & 8.71432800 & -0.49117100 \\
\hline $\mathrm{H}$ & 1.67668900 & 6.14857700 & 0.07333500 \\
\hline $\mathrm{H}$ & 0.42442200 & 6.84509700 & 1.10215800 \\
\hline $\mathrm{H}$ & -0.00825900 & 6.18528400 & -0.48278700 \\
\hline $\mathrm{H}$ & 2.72767500 & 10.47190700 & -1.15593200 \\
\hline $\mathrm{H}$ & 1.32730800 & 10.92947700 & -0.15920700 \\
\hline
\end{tabular}




$\begin{array}{lrrr}\mathrm{H} & 2.95663100 & 10.89612000 & 0.54771700 \\ \mathrm{H} & 1.79588500 & 8.87031200 & 1.31491700 \\ \mathrm{H} & 3.04510200 & 8.38236600 & 0.17755800 \\ \mathrm{H} & 1.96156400 & 1.95782700 & -2.42392000 \\ \mathrm{H} & 1.24227800 & 0.74370400 & -3.28985900 \\ \mathrm{H} & 0.27745900 & 3.67926800 & -5.12974700 \\ \mathrm{H} & 0.61413500 & 2.63687600 & -3.85683000 \\ \mathrm{H} & -0.88347500 & 4.34025300 & -3.32013300 \\ \mathrm{H} & -2.31973700 & 4.89571400 & -2.76683900 \\ \mathrm{H} & -1.29621700 & 2.06815900 & -4.65231500 \\ \mathrm{H} & -1.53627700 & 0.54324800 & -4.17174400 \\ \mathrm{H} & 0.99881900 & 2.86399800 & 0.36286600 \\ \mathrm{H} & -1.42856000 & -1.22941200 & 0.36811000 \\ \mathrm{C} & -0.58706400 & 1.67966600 & -0.28689100 \\ \mathrm{C} & -0.81179500 & 2.84650500 & -0.61237600 \\ \mathrm{H} & -0.44186600 & 0.57719900 & -0.10296400 \\ \mathrm{H} & -1.01134300 & 3.81742500 & -1.03493500\end{array}$

XN-TS,$E=-5429.652920$

$\begin{array}{lrrr}\mathrm{N} & 5.60354800 & 1.95914500 & 0.27669700 \\ \mathrm{C} & 5.10716500 & 3.21697500 & 0.89572100 \\ \mathrm{C} & 4.36300100 & 3.01513700 & 2.24734100 \\ \mathrm{O} & 4.16920000 & 3.97063500 & 3.01859100 \\ \mathrm{C} & 4.18271800 & 4.09825200 & -0.03551000 \\ \mathrm{C} & 2.90759700 & 3.35130700 & -0.37343400 \\ \mathrm{O} & 1.84933600 & 3.69636400 & 0.39407900 \\ \mathrm{O} & 2.87901100 & 2.44565800 & -1.24211700 \\ \mathrm{~N} & 0.29662700 & 7.05537200 & -3.95824300 \\ \mathrm{C} & 1.43174400 & 7.76541200 & -3.35819300 \\ \mathrm{C} & 2.73157600 & 6.94905600 & -3.46482600 \\ \mathrm{O} & 3.80907700 & 7.38114400 & -3.92875400 \\ \mathrm{C} & 1.12105000 & 8.17137300 & -1.88439100 \\ \mathrm{C} & 2.19240400 & 9.11721800 & -1.27883100 \\ \mathrm{C} & 0.90251300 & 6.94686100 & -0.96515900 \\ \mathrm{C} & 2.34701200 & 10.47532000 & -1.99842900 \\ \mathrm{~N} & -2.86717400 & -0.07170200 & -8.04245500 \\ \mathrm{C} & -3.97369300 & -1.03695300 & -7.99003600 \\ \mathrm{C} & -4.78253100 & -1.08673200 & -6.66959200 \\ \mathrm{O} & -5.80716400 & -1.83686200 & -6.57985800 \\ \mathrm{C} & -3.48298300 & -2.43701400 & -8.40963000 \\ \mathrm{~N} & -4.41749500 & -0.24592300 & -5.65852900 \\ \mathrm{C} & -5.12294700 & -0.29287100 & -4.36059500 \\ \mathrm{C} & -6.62600000 & -0.46242900 & -4.56296100 \\ \mathrm{O} & -7.35961400 & -1.06027300 & -3.74814900 \\ \mathrm{C} & -4.82537500 & 0.99513100 & -3.52859400 \\ \mathrm{C} & -5.71125800 & 2.21286000 & -3.84947000 \\ \mathrm{~S} & -5.14045200 & 3.76662300 & -2.99626200 \\ \mathrm{C} & -5.41226800 & 3.28740100 & -1.22990500 \\ \mathrm{~N} & -5.72072000 & -3.27447400 & -2.29475000 \\ \mathrm{C} & -4.85541700 & -3.06268900 & -1.11739200 \\ & & & 570 \\ & & & \end{array}$




\begin{tabular}{|c|c|c|c|}
\hline $\mathrm{C}$ & -5.28380000 & -1.73121700 & -0.44109900 \\
\hline $\mathrm{O}$ & -5.03164100 & -0.60646400 & -0.95738500 \\
\hline $\mathrm{C}$ & -3.35576600 & -3.05176300 & -1.44060600 \\
\hline S & -2.35456400 & -3.30718000 & 0.10328300 \\
\hline $\mathrm{N}$ & -5.99857800 & -1.81826200 & 0.73260000 \\
\hline $\mathrm{C}$ & -6.40502500 & -0.65310900 & 1.56333700 \\
\hline $\mathrm{C}$ & -7.44318400 & 0.21978100 & 0.83637700 \\
\hline $\mathrm{O}$ & -8.43790800 & 0.70907700 & 1.40702900 \\
\hline $\mathrm{C}$ & -5.18325900 & 0.20556200 & 2.06587400 \\
\hline $\mathrm{C}$ & -4.19928400 & -0.70388400 & 2.84844300 \\
\hline $\mathrm{C}$ & -5.67016600 & 1.39419700 & 2.92665800 \\
\hline $\mathrm{C}$ & -2.87407500 & -0.01022100 & 3.22715900 \\
\hline $\mathrm{N}$ & 9.11557300 & -3.21240900 & 5.04757600 \\
\hline $\mathrm{C}$ & 8.70994700 & -2.04908500 & 5.87619700 \\
\hline $\mathrm{C}$ & 9.31410100 & -2.22479100 & 7.27643200 \\
\hline $\mathrm{O}$ & 8.88296300 & -1.70216200 & 8.32018200 \\
\hline $\mathrm{C}$ & 7.19320000 & -1.72927100 & 5.96739000 \\
\hline $\mathrm{C}$ & 6.53343200 & -1.19485200 & 4.71630500 \\
\hline $\mathrm{C}$ & 7.06310200 & -0.44583600 & 3.67128800 \\
\hline $\mathrm{C}$ & 5.11485000 & -1.33662900 & 4.43823800 \\
\hline $\mathrm{N}$ & 6.05355900 & -0.12183400 & 2.75292600 \\
\hline $\mathrm{C}$ & 4.84819600 & -0.66785900 & 3.20243000 \\
\hline $\mathrm{C}$ & 4.05312900 & -1.98450100 & 5.11636900 \\
\hline $\mathrm{C}$ & 3.56641500 & -0.66518600 & 2.61452300 \\
\hline $\mathrm{C}$ & 2.77502200 & -1.97163600 & 4.54150400 \\
\hline $\mathrm{C}$ & 2.53736300 & -1.32985800 & 3.29433500 \\
\hline $\mathrm{N}$ & -3.12260600 & -2.67726200 & 9.28174000 \\
\hline $\mathrm{C}$ & -3.77136500 & -3.64136100 & 8.38073800 \\
\hline $\mathrm{C}$ & -2.78996900 & -4.63619400 & 7.74323000 \\
\hline $\mathrm{O}$ & -1.61044100 & -4.76229000 & 8.12719600 \\
\hline $\mathrm{C}$ & -4.62750100 & -2.89657500 & 7.30981700 \\
\hline $\mathrm{C}$ & -5.55909600 & -3.77871700 & 6.51320600 \\
\hline $\mathrm{C}$ & -6.81005900 & -4.22107800 & 6.91762200 \\
\hline $\mathrm{C}$ & -5.34162800 & -4.31449400 & 5.17771800 \\
\hline $\mathrm{N}$ & -7.38347100 & -5.00733800 & 5.91188300 \\
\hline $\mathrm{C}$ & -6.50273600 & -5.07451400 & 4.82741000 \\
\hline $\mathrm{C}$ & -4.28667000 & -4.20261100 & 4.23788500 \\
\hline $\mathrm{C}$ & -6.63562700 & -5.71624500 & 3.58073400 \\
\hline $\mathrm{C}$ & -4.40897100 & -4.83441500 & 2.99300700 \\
\hline $\mathrm{C}$ & -5.57499800 & -5.58441600 & 2.67022700 \\
\hline $\mathrm{N}$ & -2.72069100 & 9.74170000 & 1.53250500 \\
\hline $\mathrm{C}$ & -3.50921900 & 8.80861400 & 2.35478100 \\
\hline $\mathrm{C}$ & -4.76593800 & 9.61550600 & 2.67503500 \\
\hline $\mathrm{O}$ & -5.16679800 & 9.90915300 & 3.81743500 \\
\hline $\mathrm{C}$ & -2.81293500 & 8.23003300 & 3.60986900 \\
\hline $\mathrm{C}$ & -1.56420100 & 7.41955100 & 3.33354200 \\
\hline $\mathrm{C}$ & -1.30827200 & 6.53827900 & 2.28913800 \\
\hline $\mathrm{C}$ & -0.39283900 & 7.38071700 & 4.19201100 \\
\hline $\mathrm{N}$ & -0.04563700 & 5.95657400 & 2.45222000 \\
\hline $\mathrm{C}$ & 0.53901100 & 6.45771200 & 3.61402500 \\
\hline
\end{tabular}




\begin{tabular}{|c|c|c|c|}
\hline C & -0.04851600 & 8.03891600 & 5.39822000 \\
\hline $\mathrm{C}$ & 1.78890000 & 6.17541600 & 4.20086300 \\
\hline $\mathrm{C}$ & 1.19469600 & 7.76817100 & 5.98613700 \\
\hline $\mathrm{C}$ & 2.10173700 & 6.84519000 & 5.39297200 \\
\hline $\mathrm{N}$ & 10.13638800 & -3.58760100 & -4.93311100 \\
\hline $\mathrm{C}$ & 8.85970800 & -3.07209300 & -4.49018000 \\
\hline $\mathrm{C}$ & 7.86902300 & -3.34702500 & -5.59598800 \\
\hline $\mathrm{O}$ & 8.14507900 & -4.03177300 & -6.60282600 \\
\hline $\mathrm{C}$ & 8.93106800 & -1.54943700 & -4.12253400 \\
\hline $\mathrm{C}$ & 7.58544200 & -0.86782000 & -3.91543700 \\
\hline $\mathrm{C}$ & 6.64200800 & -1.34774300 & -2.97590700 \\
\hline $\mathrm{C}$ & 7.23560800 & 0.24712000 & -4.70936100 \\
\hline $\mathrm{C}$ & 5.37188200 & -0.75160500 & -2.85420600 \\
\hline $\mathrm{C}$ & 5.97450600 & 0.85979200 & -4.57848100 \\
\hline $\mathrm{C}$ & 5.03445600 & 0.35612600 & -3.65954800 \\
\hline $\mathrm{C}$ & -0.50092100 & -2.37235100 & -5.68882400 \\
\hline $\mathrm{O}$ & -0.44896900 & -3.49283400 & -6.59639800 \\
\hline $\mathrm{C}$ & -0.41999000 & -2.85480400 & -4.27161800 \\
\hline $\mathrm{S}$ & 0.07579200 & -1.67060400 & -3.04716700 \\
\hline $\mathrm{C}$ & -0.63254800 & -4.15718700 & -3.93414400 \\
\hline $\mathrm{S}$ & -0.46324000 & -4.71650100 & -2.26327300 \\
\hline $\mathrm{C}$ & -0.97967200 & -5.22367000 & -4.95963800 \\
\hline $\mathrm{C}$ & -1.39501400 & -4.55603500 & -6.26988800 \\
\hline $\mathrm{C}$ & 2.94101900 & -5.34872700 & 1.92792800 \\
\hline $\mathrm{O}$ & 4.39309800 & -5.48944900 & 1.78029700 \\
\hline $\mathrm{C}$ & 2.34491600 & -4.31760800 & 0.98791900 \\
\hline $\mathrm{S}$ & 0.59922200 & -4.48179700 & 0.66851000 \\
\hline $\mathrm{C}$ & 3.10041100 & -3.38381100 & 0.35345800 \\
\hline $\mathrm{S}$ & 2.34262900 & -2.17253100 & -0.73844500 \\
\hline $\mathrm{C}$ & 4.61570700 & -3.37043600 & 0.49502200 \\
\hline $\mathrm{C}$ & 5.05918600 & -4.18646600 & 1.72589200 \\
\hline W & 0.00332300 & -2.79890800 & -0.93470000 \\
\hline $\mathrm{O}$ & 1.61435400 & 1.14187100 & -3.25277100 \\
\hline $\mathrm{O}$ & -1.79461600 & 0.62721800 & -4.93372200 \\
\hline $\mathrm{O}$ & -1.01409100 & 4.61285200 & -3.23274600 \\
\hline $\mathrm{O}$ & 0.08306800 & 2.57921000 & -4.59808600 \\
\hline $\mathrm{O}$ & -0.60900300 & -1.06388100 & -0.10838400 \\
\hline $\mathrm{H}$ & 6.86076800 & -2.92430500 & -5.46577100 \\
\hline $\mathrm{H}$ & 7.94787000 & 0.62576600 & -5.44123600 \\
\hline $\mathrm{H}$ & 5.71926100 & 1.71316500 & -5.20363800 \\
\hline $\mathrm{H}$ & 4.04571000 & 0.79917600 & -3.58847100 \\
\hline $\mathrm{H}$ & 4.63539100 & -1.16431900 & -2.16591600 \\
\hline $\mathrm{H}$ & 6.88480500 & -2.20295500 & -2.34643000 \\
\hline $\mathrm{H}$ & 9.55029000 & -1.47599400 & -3.21578300 \\
\hline $\mathrm{H}$ & 9.48859400 & -1.05704300 & -4.92748500 \\
\hline $\mathrm{H}$ & 10.08992500 & -4.20148200 & -5.74051000 \\
\hline $\mathrm{H}$ & 10.81339000 & -3.83627300 & -4.22385700 \\
\hline $\mathrm{H}$ & 8.44858400 & -3.60237800 & -3.60572700 \\
\hline H & -2.41219600 & -4.14078800 & -6.17485700 \\
\hline $\mathrm{H}$ & -1.36633000 & -5.24406600 & -7.12216600 \\
\hline
\end{tabular}




\begin{tabular}{|c|c|c|c|}
\hline $\mathrm{H}$ & -0.10446600 & -5.87052300 & -5.12156100 \\
\hline $\mathrm{H}$ & -1.78929100 & -5.86543900 & -4.58231800 \\
\hline $\mathrm{H}$ & 0.35543500 & -1.73882700 & -5.94766000 \\
\hline $\mathrm{H}$ & -1.42316900 & -1.78970100 & -5.86629600 \\
\hline $\mathrm{H}$ & 2.71169200 & -5.08916500 & 2.97796500 \\
\hline $\mathrm{H}$ & 2.53221000 & -6.34357500 & 1.72059000 \\
\hline $\mathrm{H}$ & 4.98984800 & -2.34099200 & 0.59933200 \\
\hline $\mathrm{H}$ & 5.06956400 & -3.79166100 & -0.41779100 \\
\hline $\mathrm{H}$ & 4.83882000 & -3.62379000 & 2.64594700 \\
\hline $\mathrm{H}$ & 6.13184900 & -4.41417800 & 1.69159000 \\
\hline $\mathrm{H}$ & -3.19693800 & -5.28031600 & 6.94285000 \\
\hline $\mathrm{H}$ & -7.52509600 & -6.28836800 & 3.32482100 \\
\hline $\mathrm{H}$ & -5.63817900 & -6.06325300 & 1.69582100 \\
\hline $\mathrm{H}$ & -3.61895100 & -4.73378200 & 2.25205800 \\
\hline $\mathrm{H}$ & -3.39294100 & -3.62435100 & 4.46100800 \\
\hline $\mathrm{H}$ & -8.30023500 & -5.42797500 & 5.94759600 \\
\hline $\mathrm{H}$ & -7.33865400 & -4.02317200 & 7.83984100 \\
\hline $\mathrm{H}$ & -3.95455900 & -2.35223600 & 6.63159600 \\
\hline $\mathrm{H}$ & -5.20047000 & -2.13939000 & 7.86058800 \\
\hline $\mathrm{H}$ & -2.80406800 & -1.83883400 & 8.79619600 \\
\hline $\mathrm{H}$ & -2.35040600 & -3.09476000 & 9.80182300 \\
\hline $\mathrm{H}$ & -4.47134100 & -4.26437900 & 8.96464600 \\
\hline $\mathrm{H}$ & -5.28307400 & 9.98893700 & 1.77015600 \\
\hline $\mathrm{H}$ & -2.10165900 & 9.30117500 & 0.85800400 \\
\hline $\mathrm{H}$ & -2.24237400 & 10.46046100 & 2.07126700 \\
\hline $\mathrm{H}$ & -3.82605900 & 7.98903000 & 1.69151700 \\
\hline $\mathrm{H}$ & -2.56433000 & 9.06622000 & 4.27836300 \\
\hline $\mathrm{H}$ & -3.55128400 & 7.62836100 & 4.16161100 \\
\hline $\mathrm{H}$ & -1.92622400 & 6.26471200 & 1.44519000 \\
\hline $\mathrm{H}$ & 0.39604700 & 5.30842100 & 1.80927000 \\
\hline $\mathrm{H}$ & 2.48360600 & 5.46236200 & 3.76518400 \\
\hline $\mathrm{H}$ & 3.05543100 & 6.64643900 & 5.87692700 \\
\hline $\mathrm{H}$ & 1.47311300 & 8.26250800 & 6.91490200 \\
\hline $\mathrm{H}$ & -0.73869600 & 8.73714800 & 5.86780000 \\
\hline $\mathrm{H}$ & -3.07771400 & -2.11295000 & -1.93243800 \\
\hline $\mathrm{H}$ & -3.13024700 & -3.88034500 & -2.12323900 \\
\hline $\mathrm{H}$ & -6.29566800 & -2.50199200 & -2.62619200 \\
\hline $\mathrm{H}$ & -5.30269800 & -3.82728300 & -3.03557600 \\
\hline $\mathrm{H}$ & -5.02410200 & -3.87669000 & -0.39109800 \\
\hline $\mathrm{H}$ & -6.13282600 & -2.74180300 & 1.12986100 \\
\hline $\mathrm{H}$ & -6.90267900 & -1.06107900 & 2.45436700 \\
\hline $\mathrm{H}$ & -4.66290100 & 0.58962000 & 1.17930900 \\
\hline $\mathrm{H}$ & -6.32182400 & 2.07705900 & 2.36954800 \\
\hline $\mathrm{H}$ & -6.23418200 & 1.04135200 & 3.80247300 \\
\hline $\mathrm{H}$ & -4.81387600 & 1.97509500 & 3.28788600 \\
\hline $\mathrm{H}$ & -3.01922100 & 0.82016700 & 3.93135400 \\
\hline $\mathrm{H}$ & -2.18514400 & -0.72834500 & 3.69083100 \\
\hline $\mathrm{H}$ & -2.37507400 & 0.37956000 & 2.33086300 \\
\hline $\mathrm{H}$ & -4.70312400 & -1.07276200 & 3.75831300 \\
\hline $\mathrm{H}$ & -3.95367800 & -1.57980700 & 2.2364810 \\
\hline
\end{tabular}




\begin{tabular}{|c|c|c|c|}
\hline $\mathrm{H}$ & -7.24688200 & 0.39722600 & -0.23392200 \\
\hline $\mathrm{H}$ & 4.00840900 & 1.99789900 & 2.48137400 \\
\hline $\mathrm{H}$ & 6.41487300 & 2.10867700 & -0.32036900 \\
\hline $\mathrm{H}$ & 4.88468600 & 1.43933300 & -0.23200400 \\
\hline $\mathrm{H}$ & 5.97971300 & 3.84109900 & 1.12911100 \\
\hline $\mathrm{H}$ & 3.94365900 & 5.02894300 & 0.48529600 \\
\hline $\mathrm{H}$ & 4.72464600 & 4.31188100 & -0.96248000 \\
\hline $\mathrm{H}$ & 10.21347000 & -2.87400000 & 7.28314800 \\
\hline $\mathrm{H}$ & 9.05092900 & -3.02411600 & 4.04891500 \\
\hline $\mathrm{H}$ & 8.59763400 & -4.06104100 & 5.27805400 \\
\hline $\mathrm{H}$ & 9.23024500 & -1.17108300 & 5.45543900 \\
\hline 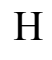 & 6.67385300 & -2.64980500 & 6.27643100 \\
\hline $\mathrm{H}$ & 7.05658100 & -1.02166500 & 6.79844200 \\
\hline $\mathrm{H}$ & 8.07772900 & -0.10779700 & 3.50854900 \\
\hline $\mathrm{H}$ & 6.14618400 & 0.45468300 & 1.91395400 \\
\hline $\mathrm{H}$ & 3.37353100 & -0.24284400 & 1.63225100 \\
\hline $\mathrm{H}$ & 1.55617700 & -1.38702900 & 2.83207700 \\
\hline $\mathrm{H}$ & 1.95239500 & -2.48219400 & 5.03647000 \\
\hline $\mathrm{H}$ & 4.22438600 & -2.49561200 & 6.06175400 \\
\hline $\mathrm{H}$ & -7.06158800 & 0.07839700 & -5.42185100 \\
\hline $\mathrm{H}$ & -2.59384300 & -2.72882000 & -7.84218800 \\
\hline $\mathrm{H}$ & -4.27332400 & -3.17801900 & -8.25144700 \\
\hline $\mathrm{H}$ & -3.20436700 & -2.41794300 & -9.46944500 \\
\hline $\mathrm{H}$ & -2.05435900 & -0.32150400 & -7.48264100 \\
\hline $\mathrm{H}$ & -3.13649700 & 0.89853800 & -7.90045700 \\
\hline $\mathrm{H}$ & -4.72337000 & -0.72143100 & -8.73241400 \\
\hline $\mathrm{H}$ & -3.48502400 & 0.19520300 & -5.63923900 \\
\hline $\mathrm{H}$ & -4.76965800 & -1.14719000 & -3.76843800 \\
\hline $\mathrm{H}$ & -4.93946900 & 0.71326600 & -2.48302500 \\
\hline $\mathrm{H}$ & -3.77332400 & 1.25550600 & -3.68949700 \\
\hline $\mathrm{H}$ & -5.68202200 & 2.46797700 & -4.91840200 \\
\hline $\mathrm{H}$ & -6.75900600 & 2.04488000 & -3.56848000 \\
\hline $\mathrm{H}$ & -5.13379100 & 4.15416500 & -0.61965100 \\
\hline $\mathrm{H}$ & -6.46865000 & 3.05093800 & -1.05792100 \\
\hline $\mathrm{H}$ & -4.79064700 & 2.43208700 & -0.95204700 \\
\hline $\mathrm{H}$ & 2.63505100 & 5.88592500 & -3.15791600 \\
\hline $\mathrm{H}$ & 0.30547300 & 7.00115600 & -4.97122100 \\
\hline $\mathrm{H}$ & -0.12663300 & 6.23166900 & -3.50935500 \\
\hline $\mathrm{H}$ & 1.60297400 & 8.68769500 & -3.92928900 \\
\hline $\mathrm{H}$ & 0.16926600 & 8.72254500 & -1.95110000 \\
\hline $\mathrm{H}$ & 1.84059500 & 6.40279800 & -0.78887700 \\
\hline $\mathrm{H}$ & 0.53538900 & 7.27617300 & 0.01502800 \\
\hline $\mathrm{H}$ & 0.17813200 & 6.23669900 & -1.38083300 \\
\hline $\mathrm{H}$ & 2.76664800 & 10.35398400 & -3.00437500 \\
\hline $\mathrm{H}$ & 1.37653500 & 10.98365100 & -2.09248400 \\
\hline $\mathrm{H}$ & 3.02134000 & 11.13737400 & -1.43774500 \\
\hline $\mathrm{H}$ & 1.91916700 & 9.29892100 & -0.22816900 \\
\hline $\mathrm{H}$ & 3.16744900 & 8.60683100 & -1.25718400 \\
\hline $\mathrm{H}$ & 2.07597000 & 1.47748300 & -2.44594600 \\
\hline $\mathrm{H}$ & 1.41175200 & 0.16946100 & -3.25321200 \\
\hline
\end{tabular}




$\begin{array}{lrrr}\mathrm{H} & 0.49114200 & 2.88579700 & -5.42879900 \\ \mathrm{H} & 0.75219200 & 2.00048100 & -4.03365200 \\ \mathrm{H} & -0.61380900 & 3.82686900 & -3.70849100 \\ \mathrm{H} & -1.96347800 & 4.46434200 & -3.05620500 \\ \mathrm{H} & -1.19318800 & 1.42168500 & -4.87386000 \\ \mathrm{H} & -1.49231000 & -0.04746800 & -4.28136500 \\ \mathrm{H} & 1.04018000 & 3.03896400 & 0.36973200 \\ \mathrm{H} & -1.47773000 & -1.15688500 & 0.35155800 \\ \mathrm{C} & -0.04280700 & 1.14548400 & -0.09332300 \\ \mathrm{C} & -0.20579000 & 1.89588400 & 0.88743100 \\ \mathrm{H} & 0.22328900 & 0.76743600 & -1.05663300 \\ \mathrm{H} & -0.52882200 & 2.11276300 & 1.88628300\end{array}$

XN-EP1,$E=-5429.750747$

$\begin{array}{llcc}\mathrm{N} & -5.64983500 & 1.68790100 & -0.85384600 \\ \mathrm{C} & -5.16885600 & 2.75892200 & -1.79356800 \\ \mathrm{C} & -4.40251400 & 2.21678800 & -3.02235100 \\ \mathrm{O} & -4.50492900 & 2.74322800 & -4.14583800 \\ \mathrm{C} & -4.22878000 & 3.83507400 & -1.13884300 \\ \mathrm{C} & -2.85114700 & 3.26504800 & -0.72089100 \\ \mathrm{O} & -1.79403400 & 3.70016100 & -1.29253400 \\ \mathrm{O} & -2.90647300 & 2.34062800 & 0.20641600 \\ \mathrm{~N} & -0.49472600 & 7.82762000 & 1.93885700 \\ \mathrm{C} & -1.66395000 & 8.40667800 & 1.26245100 \\ \mathrm{C} & -2.92048500 & 7.54392800 & 1.47097100 \\ \mathrm{O} & -3.98403600 & 7.95997200 & 1.98232800 \\ \mathrm{C} & -1.37033900 & 8.66547600 & -0.24640900 \\ \mathrm{C} & -2.54384700 & 9.37519800 & -0.97391000 \\ \mathrm{C} & -0.96317600 & 7.38105800 & -1.00230300 \\ \mathrm{C} & -2.94303000 & 10.74827000 & -0.38862900 \\ \mathrm{~N} & 2.77181900 & 2.12509200 & 7.80422000 \\ \mathrm{C} & 3.86959200 & 1.16754600 & 7.98641900 \\ \mathrm{C} & 4.68441900 & 0.81366300 & 6.71892300 \\ \mathrm{O} & 5.72468200 & 0.08474300 & 6.81400700 \\ \mathrm{C} & 3.35452600 & -0.10851600 & 8.68412200 \\ \mathrm{~N} & 4.29932000 & 1.35265400 & 5.52582700 \\ \mathrm{C} & 5.00663700 & 0.97189300 & 4.28557600 \\ \mathrm{C} & 6.51010300 & 0.86168600 & 4.52879900 \\ \mathrm{O} & 7.23998400 & 0.05889400 & 3.90880400 \\ \mathrm{C} & 4.71376100 & 1.99783700 & 3.14554300 \\ \mathrm{C} & 5.60224300 & 3.25485200 & 3.15260400 \\ \mathrm{~S} & 5.06393500 & 4.53589800 & 1.91541600 \\ \mathrm{C} & 5.42975500 & 3.64097100 & 0.33885100 \\ \mathrm{~N} & 5.69339000 & -2.43893800 & 3.10665800 \\ \mathrm{C} & 4.88033200 & -2.56930700 & 1.89307200 \\ \mathrm{C} & 5.29213500 & -1.45337200 & 0.88872600 \\ \mathrm{O} & 4.96511900 & -0.24613700 & 1.06618200 \\ \mathrm{C} & 3.36217300 & -2.50033000 & 2.14748000 \\ \mathrm{~S} & 2.40439500 & -3.02390600 & 0.62759500\end{array}$




\begin{tabular}{|c|c|c|c|}
\hline $\mathrm{N}$ & 6.04006300 & -1.83276500 & -0.20051700 \\
\hline $\mathrm{C}$ & 6.44504200 & -0.93291800 & -1.31597200 \\
\hline $\mathrm{C}$ & 7.43309800 & 0.14071600 & -0.83226100 \\
\hline $\mathrm{O}$ & 8.47743200 & 0.42757000 & -1.44817000 \\
\hline 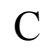 & 5.22864200 & -0.28380500 & -2.07503400 \\
\hline $\mathrm{C}$ & 4.30209200 & -1.39982000 & -2.62493600 \\
\hline $\mathrm{C}$ & 5.73090500 & 0.64928400 & -3.20167800 \\
\hline $\mathrm{C}$ & 3.00711100 & -0.87516900 & -3.28157100 \\
\hline $\mathrm{N}$ & -9.00183900 & -4.62672400 & -4.12943600 \\
\hline $\mathrm{C}$ & -8.61558900 & -3.70377000 & -5.22799900 \\
\hline $\mathrm{C}$ & -9.18868900 & -4.26219800 & -6.54082800 \\
\hline $\mathrm{O}$ & -8.73233400 & -4.05111700 & -7.67872600 \\
\hline $\mathrm{C}$ & -7.10880000 & -3.37206800 & -5.38851000 \\
\hline $\mathrm{C}$ & -6.45749900 & -2.51485000 & -4.32559700 \\
\hline $\mathrm{C}$ & -6.98180200 & -1.53105800 & -3.49209900 \\
\hline $\mathrm{C}$ & -5.02725000 & -2.53220000 & -4.08009200 \\
\hline $\mathrm{N}$ & -5.95847300 & -0.94454300 & -2.73343400 \\
\hline $\mathrm{C}$ & -4.74825700 & -1.54693500 & -3.08127400 \\
\hline $\mathrm{C}$ & -3.96340100 & -3.29880400 & -4.61607100 \\
\hline $\mathrm{C}$ & -3.44412700 & -1.33395400 & -2.58964400 \\
\hline $\mathrm{C}$ & -2.66487700 & -3.08110700 & -4.13729700 \\
\hline $\mathrm{C}$ & -2.41149600 & -2.11336000 & -3.12604400 \\
\hline $\mathrm{N}$ & 3.28110900 & -4.94903400 & -8.24966900 \\
\hline $\mathrm{C}$ & 3.93425000 & -5.63988900 & -7.12917100 \\
\hline $\mathrm{C}$ & 2.96290700 & -6.45351700 & -6.26098900 \\
\hline $\mathrm{O}$ & 1.78339800 & -6.68155100 & -6.59329100 \\
\hline $\mathrm{C}$ & 4.77094200 & -4.63051300 & -6.28269900 \\
\hline $\mathrm{C}$ & 5.71618000 & -5.26090500 & -5.28791700 \\
\hline $\mathrm{C}$ & 6.98108200 & -5.75776000 & -5.56517700 \\
\hline $\mathrm{C}$ & 5.50115200 & -5.45078600 & -3.86148900 \\
\hline $\mathrm{N}$ & 7.56526500 & -6.25203400 & -4.39341700 \\
\hline $\mathrm{C}$ & 6.67766800 & -6.06882400 & -3.32911100 \\
\hline $\mathrm{C}$ & 4.43556300 & -5.13569700 & -2.98247600 \\
\hline $\mathrm{C}$ & 6.81477400 & -6.37582700 & -1.96155400 \\
\hline $\mathrm{C}$ & 4.56260000 & -5.43425300 & -1.61918300 \\
\hline $\mathrm{C}$ & 5.74292500 & -6.04959000 & -1.11534200 \\
\hline $\mathrm{N}$ & 2.54578700 & 9.04468600 & -4.03966900 \\
\hline $\mathrm{C}$ & 3.54677800 & 7.97949300 & -4.22489100 \\
\hline $\mathrm{C}$ & 4.60806300 & 8.66695300 & -5.09252100 \\
\hline $\mathrm{O}$ & 4.98435700 & 8.29450900 & -6.22029600 \\
\hline $\mathrm{C}$ & 3.05755100 & 6.60930300 & -4.79862400 \\
\hline $\mathrm{C}$ & 1.70187200 & 6.12557200 & -4.32534700 \\
\hline $\mathrm{C}$ & 1.38341900 & 5.34223600 & -3.21528900 \\
\hline $\mathrm{C}$ & 0.45438600 & 6.32241800 & -5.04165500 \\
\hline $\mathrm{N}$ & 0.02281300 & 5.04085100 & -3.20341300 \\
\hline $\mathrm{C}$ & -0.57072000 & 5.62818900 & -4.31520300 \\
\hline $\mathrm{C}$ & 0.11123800 & 6.99995000 & -6.23843700 \\
\hline $\mathrm{C}$ & -1.90735100 & 5.58673900 & -4.76028000 \\
\hline $\mathrm{C}$ & -1.22012800 & 6.97262900 & -6.67711500 \\
\hline $\mathrm{C}$ & -2.21889400 & 6.26996700 & -5.94466000 \\
\hline
\end{tabular}




\begin{tabular}{|c|c|c|c|}
\hline N & -10.14897800 & -2.39474900 & 5.59163900 \\
\hline $\mathrm{C}$ & -8.88168800 & -1.96282500 & 5.04535900 \\
\hline $\mathrm{C}$ & -7.89549200 & -1.94066400 & 6.18650300 \\
\hline $\mathrm{O}$ & -8.15530100 & -2.38402800 & 7.32445800 \\
\hline C & -8.97857300 & -0.56929300 & 4.33444900 \\
\hline $\mathrm{C}$ & -7.65204700 & 0.02112800 & 3.87223400 \\
\hline $\mathrm{C}$ & -6.78966300 & -0.67988400 & 2.99609600 \\
\hline $\mathrm{C}$ & -7.24704300 & 1.29403700 & 4.33250500 \\
\hline $\mathrm{C}$ & -5.55277700 & -0.13221300 & 2.60483600 \\
\hline $\mathrm{C}$ & -6.01774500 & 1.85214700 & 3.93068800 \\
\hline $\mathrm{C}$ & -5.16068200 & 1.14009300 & 3.06962500 \\
\hline $\mathrm{C}$ & 0.28578300 & -0.85784800 & 6.03313300 \\
\hline $\mathrm{O}$ & 0.40963800 & -1.64217200 & 7.25730900 \\
\hline $\mathrm{C}$ & 0.26115800 & -1.71774200 & 4.78904300 \\
\hline $\mathrm{S}$ & -0.14455100 & -0.92490100 & 3.25018500 \\
\hline $\mathrm{C}$ & 0.49370100 & -3.05931600 & 4.81501500 \\
\hline $\mathrm{S}$ & 0.47626200 & -4.02208100 & 3.32084900 \\
\hline $\mathrm{C}$ & 0.79765200 & -3.78788100 & 6.11412100 \\
\hline $\mathrm{C}$ & 1.31830800 & -2.78361400 & 7.14977900 \\
\hline $\mathrm{C}$ & -2.84200700 & -5.81383000 & -0.57690300 \\
\hline $\mathrm{O}$ & -4.28235000 & -5.86688800 & -0.34126000 \\
\hline $\mathrm{C}$ & -2.23444900 & -4.54624100 & -0.01301200 \\
\hline $\mathrm{S}$ & -0.51053600 & -4.59499600 & 0.41153700 \\
\hline $\mathrm{C}$ & -2.99224500 & -3.45370400 & 0.27551400 \\
\hline $\mathrm{S}$ & -2.23776700 & -2.00142100 & 0.97883700 \\
\hline $\mathrm{C}$ & -4.50214200 & -3.44695600 & 0.09868900 \\
\hline $\mathrm{C}$ & -4.94621100 & -4.63512200 & -0.77164100 \\
\hline W & 0.01949500 & -2.57028400 & 1.50512500 \\
\hline $\mathrm{O}$ & -1.71588900 & 2.01622300 & 2.43325100 \\
\hline $\mathrm{O}$ & 1.64695600 & 1.94840300 & 4.55372700 \\
\hline $\mathrm{O}$ & 1.02509100 & 5.37789800 & 1.83460800 \\
\hline $\mathrm{O}$ & -0.16985100 & 3.62101200 & 3.50905000 \\
\hline $\mathrm{O}$ & 0.65892800 & -0.87520000 & 0.25281800 \\
\hline $\mathrm{H}$ & -6.90298400 & -1.51811100 & 5.96221200 \\
\hline $\mathrm{H}$ & -7.89540200 & 1.84753900 & 5.01054600 \\
\hline $\mathrm{H}$ & -5.72412000 & 2.83458400 & 4.29424400 \\
\hline $\mathrm{H}$ & -4.20280200 & 1.55999700 & 2.77493000 \\
\hline $\mathrm{H}$ & -4.88283000 & -0.69604400 & 1.95980200 \\
\hline $\mathrm{H}$ & -7.07758200 & -1.66014900 & 2.61783300 \\
\hline $\mathrm{H}$ & -9.65888000 & -0.70563800 & 3.48037100 \\
\hline $\mathrm{H}$ & -9.48072000 & 0.11290400 & 5.03038500 \\
\hline $\mathrm{H}$ & -10.09872500 & -2.80687200 & 6.5177180 \\
\hline $\mathrm{H}$ & -10.82255000 & -2.80086000 & 4.95604600 \\
\hline $\mathrm{H}$ & -8.44961000 & -2.67303000 & 4.30938000 \\
\hline $\mathrm{H}$ & 2.32721800 & -2.44117600 & 6.86516500 \\
\hline $\mathrm{H}$ & 1.35565800 & -3.21094400 & 8.15765500 \\
\hline $\mathrm{H}$ & -0.11117400 & -4.27845400 & 6.49282600 \\
\hline $\mathrm{H}$ & 1.54559500 & -4.57586600 & 5.94451000 \\
\hline $\mathrm{H}$ & -0.64740500 & -0.29390800 & 6.14534200 \\
\hline $\mathrm{H}$ & 1.11370600 & -0.13074300 & 5.98247600 \\
\hline
\end{tabular}




\begin{tabular}{|c|c|c|c|}
\hline $\mathrm{H}$ & -2.64575300 & -5.89448700 & -1.66162600 \\
\hline $\mathrm{H}$ & -2.43016800 & -6.69922900 & -0.08149900 \\
\hline $\mathrm{H}$ & -4.83716500 & -2.51031700 & -0.36519900 \\
\hline $\mathrm{H}$ & -4.97636300 & -3.51125300 & 1.09106500 \\
\hline $\mathrm{H}$ & -4.71286800 & -4.43857800 & -1.82941400 \\
\hline $\mathrm{H}$ & -6.01925900 & -4.83210900 & -0.66923200 \\
\hline $\mathrm{H}$ & 3.37979900 & -6.87605300 & -5.32834100 \\
\hline $\mathrm{H}$ & 7.71529400 & -6.84336600 & -1.56883200 \\
\hline $\mathrm{H}$ & 5.80998700 & -6.27151100 & -0.05271700 \\
\hline $\mathrm{H}$ & 3.75994500 & -5.17505800 & -0.93322000 \\
\hline $\mathrm{H}$ & 3.53006600 & -4.65572400 & -3.34648100 \\
\hline $\mathrm{H}$ & 8.49211900 & -6.64526600 & -4.32170400 \\
\hline $\mathrm{H}$ & 7.51249500 & -5.78343400 & -6.50637100 \\
\hline $\mathrm{H}$ & 4.08461800 & -3.94370000 & -5.76659000 \\
\hline $\mathrm{H}$ & 5.33212400 & -4.02838700 & -7.00874700 \\
\hline $\mathrm{H}$ & 2.94101100 & -4.02113100 & -7.99827800 \\
\hline $\mathrm{H}$ & 2.52658200 & -5.49975400 & -8.65945700 \\
\hline $\mathrm{H}$ & 4.64810300 & -6.38072300 & -7.53019900 \\
\hline $\mathrm{H}$ & 4.98993900 & 9.60371100 & -4.64130000 \\
\hline $\mathrm{H}$ & 2.00429100 & 8.95342000 & -3.18308600 \\
\hline $\mathrm{H}$ & 1.92867000 & 9.16428400 & -4.84168500 \\
\hline $\mathrm{H}$ & 4.00756300 & 7.81674000 & -3.23821900 \\
\hline $\mathrm{H}$ & 3.04348800 & 6.69236300 & -5.89384500 \\
\hline $\mathrm{H}$ & 3.83178900 & 5.86302500 & -4.57033900 \\
\hline $\mathrm{H}$ & 2.03246900 & 4.96662700 & -2.43629300 \\
\hline $\mathrm{H}$ & -0.49384000 & 4.52505000 & -2.48096900 \\
\hline $\mathrm{H}$ & -2.66364800 & 5.02422200 & -4.22074900 \\
\hline $\mathrm{H}$ & -3.24027400 & 6.24705200 & -6.31867200 \\
\hline $\mathrm{H}$ & -1.49766100 & 7.48270600 & -7.59803900 \\
\hline $\mathrm{H}$ & 0.87099300 & 7.51826200 & -6.82255100 \\
\hline $\mathrm{H}$ & 3.07367800 & -1.48952700 & 2.45311900 \\
\hline $\mathrm{H}$ & 3.09854900 & -3.19756800 & 2.95008500 \\
\hline $\mathrm{H}$ & 6.24112900 & -1.59282700 & 3.25411600 \\
\hline $\mathrm{H}$ & 5.30242000 & -2.84933900 & 3.94664800 \\
\hline $\mathrm{H}$ & 5.08230200 & -3.54236800 & 1.41050400 \\
\hline $\mathrm{H}$ & 6.24183900 & -2.82085900 & -0.30823000 \\
\hline $\mathrm{H}$ & 6.98572100 & -1.55808800 & -2.03990100 \\
\hline $\mathrm{H}$ & 4.66084600 & 0.30836000 & -1.34560100 \\
\hline $\mathrm{H}$ & 6.34347400 & 1.47493200 & -2.82139100 \\
\hline $\mathrm{H}$ & 6.33980500 & 0.09497500 & -3.93060400 \\
\hline $\mathrm{H}$ & 4.88164400 & 1.08960300 & -3.73536400 \\
\hline $\mathrm{H}$ & 3.20122200 & -0.31956600 & -4.20772500 \\
\hline $\mathrm{H}$ & 2.33675200 & -1.70937200 & -3.52760600 \\
\hline $\mathrm{H}$ & 2.45797600 & -0.20893200 & -2.60286500 \\
\hline $\mathrm{H}$ & 4.86798700 & -2.00436100 & -3.35386200 \\
\hline $\mathrm{H}$ & 4.02243900 & -2.06926600 & -1.80301900 \\
\hline $\mathrm{H}$ & 7.15382600 & 0.65601200 & 0.10163100 \\
\hline $\mathrm{H}$ & -3.71967700 & 1.36882100 & -2.84508500 \\
\hline $\mathrm{H}$ & -6.48132700 & 1.98778400 & -0.34491400 \\
\hline $\mathrm{H}$ & -4.89902200 & 1.45134400 & -0.19326300 \\
\hline
\end{tabular}




\begin{tabular}{|c|c|c|c|}
\hline $\mathrm{H}$ & -6.04902900 & 3.28365900 & -2.18748400 \\
\hline $\mathrm{H}$ & -4.07664300 & 4.65351500 & -1.85091800 \\
\hline $\mathrm{H}$ & -4.72968100 & 4.22682100 & -0.24338200 \\
\hline & -10.08872200 & -4.89188500 & -6.38577600 \\
\hline & -8.97796500 & -4.18065800 & -3.21451000 \\
\hline & -8.44566900 & -5.48219100 & -4.11910400 \\
\hline $\mathrm{H}$ & -9.17015500 & -2.76506700 & -5.05499500 \\
\hline & -6.56370800 & -4.32715800 & -5.45321400 \\
\hline $\mathrm{H}$ & -6.98343200 & -2.89904200 & -6.37378900 \\
\hline $\mathrm{H}$ & -8.00230000 & -1.18818100 & -3.38604500 \\
\hline $\mathrm{H}$ & -6.04488500 & -0.15725700 & -2.07832500 \\
\hline $\mathrm{H}$ & -3.24033200 & -0.63274200 & -1.78556600 \\
\hline - & -1.40355500 & -1.99223500 & -2.73903000 \\
\hline $\mathrm{H}$ & -1.83668000 & -3.66802600 & -4.52794100 \\
\hline $\mathrm{H}$ & -4.14647100 & -4.04691500 & -5.38495700 \\
\hline $\mathrm{H}$ & 6.95037100 & 1.61990200 & 5.19875300 \\
\hline $\mathrm{H}$ & 2.49198100 & -0.52705800 & 8.15406600 \\
\hline $\mathrm{H}$ & 4.14938600 & -0.86005800 & 8.73305200 \\
\hline $\mathrm{H}$ & 3.02543500 & 0.14430900 & 9.69831200 \\
\hline $\mathrm{H}$ & 1.95715700 & 1.76546000 & 7.31131900 \\
\hline $\mathrm{H}$ & 3.04571100 & 3.04456900 & 7.46871900 \\
\hline $\mathrm{H}$ & 4.61239600 & 1.62999500 & 8.65372000 \\
\hline $\mathrm{H}$ & 3.36422900 & 1.76834500 & 5.39686100 \\
\hline $\mathrm{H}$ & 4.65708300 & -0.00946400 & 3.93865800 \\
\hline $\mathrm{H}$ & 4.83766400 & 1.45723300 & 2.20787700 \\
\hline $\mathrm{H}$ & 3.66111200 & 2.29191800 & 3.22586700 \\
\hline $\mathrm{H}$ & 5.55528400 & 3.77792600 & 4.11813400 \\
\hline $\mathrm{H}$ & 6.65389900 & 3.01656300 & 2.94704600 \\
\hline U & 5.15746200 & 4.31671900 & -0.47973600 \\
\hline $\mathrm{H}$ & 6.49868400 & 3.40726300 & 0.27117000 \\
\hline $\mathrm{H}$ & 4.84283000 & 2.72143300 & 0.26043300 \\
\hline $\mathrm{H}$ & -2.80506900 & 6.47420800 & 1.20098200 \\
\hline $\mathrm{H}$ & -0.50935900 & 7.89110900 & 2.95165200 \\
\hline $\mathrm{H}$ & -0.03916800 & 6.96938300 & 1.60755800 \\
\hline $\mathrm{H}$ & -1.88217700 & 9.37479400 & 1.73355400 \\
\hline $\mathrm{H}$ & -0.50408400 & 9.34751300 & -0.24433200 \\
\hline $\mathrm{H}$ & -1.78505500 & 6.65639900 & -1.06442300 \\
\hline $\mathrm{H}$ & -0.67146000 & 7.62328500 & -2.03207700 \\
\hline $\mathrm{H}$ & -0.11608200 & 6.87399500 & -0.52808800 \\
\hline $\mathrm{H}$ & -3.39351400 & 10.64585000 & 0.60605400 \\
\hline $\mathrm{H}$ & -2.06736100 & 11.40826700 & -0.30186000 \\
\hline $\mathrm{H}$ & -3.67821800 & 11.24761200 & -1.03536000 \\
\hline $\mathrm{H}$ & -2.25030200 & 9.50451500 & -2.02660300 \\
\hline $\mathrm{H}$ & -3.42556600 & 8.71611800 & -0.98426000 \\
\hline $\mathrm{H}$ & -2.12239500 & 2.13303300 & 1.49522200 \\
\hline $\mathrm{H}$ & -1.52978200 & 1.08312000 & 2.67027100 \\
\hline $\mathrm{H}$ & -0.65128100 & 4.17468600 & 4.15238800 \\
\hline $\mathrm{H}$ & -0.84121100 & 2.95179900 & 3.01314900 \\
\hline 11 & 0.59510500 & 4.66737600 & 2.39295000 \\
\hline 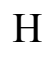 & 1.98381700 & 5.21553700 & 1.74306000 \\
\hline
\end{tabular}




$\begin{array}{rrrr}\mathrm{H} & 1.02842700 & 2.66899500 & 4.21491500 \\ \mathrm{H} & 1.40692800 & 1.11073300 & 4.09955200 \\ \mathrm{H} & 0.30257500 & 2.37818700 & -0.65090400 \\ \mathrm{H} & 1.61069000 & -1.07710300 & -0.00561100 \\ \mathrm{C} & 0.10592100 & 0.38520200 & -0.07202400 \\ \mathrm{C} & 0.83284600 & 1.46287300 & -0.40478000 \\ \mathrm{H} & -0.97598600 & 0.36555100 & -0.02442400 \\ \mathrm{H} & 1.92119200 & 1.45775900 & -0.41635800\end{array}$

XN-EP2,$E=-5429.7659306$

$\begin{array}{lccc}\mathrm{N} & -5.65064300 & 1.65583700 & -0.89796900 \\ \mathrm{C} & -5.20095800 & 2.68832900 & -1.89425900 \\ \mathrm{C} & -4.40454100 & 2.10527500 & -3.08490800 \\ \mathrm{O} & -4.48697700 & 2.58424900 & -4.23064200 \\ \mathrm{C} & -4.30304500 & 3.83074000 & -1.29429900 \\ \mathrm{C} & -2.92971600 & 3.31361800 & -0.80700800 \\ \mathrm{O} & -1.86323300 & 3.69782500 & -1.39822800 \\ \mathrm{O} & -2.99682500 & 2.47609500 & 0.19734300 \\ \mathrm{~N} & -0.48115600 & 7.88024200 & 1.67165300 \\ \mathrm{C} & -1.65354500 & 8.44750400 & 0.99285100 \\ \mathrm{C} & -2.90789900 & 7.58493700 & 1.21618300 \\ \mathrm{O} & -3.97443100 & 8.00849000 & 1.71493800 \\ \mathrm{C} & -1.36713100 & 8.68833600 & -0.52070700 \\ \mathrm{C} & -2.54332900 & 9.39125700 & -1.25050200 \\ \mathrm{C} & -0.96636300 & 7.39407900 & -1.26332500 \\ \mathrm{C} & -2.93873500 & 10.77114800 & -0.67891500 \\ \mathrm{~N} & 2.79251700 & 2.39712000 & 7.73062100 \\ \mathrm{C} & 3.87607400 & 1.42934700 & 7.94679200 \\ \mathrm{C} & 4.69945100 & 1.03474500 & 6.69783100 \\ \mathrm{O} & 5.73893500 & 0.30988600 & 6.82450900 \\ \mathrm{C} & 3.33699600 & 0.17461400 & 8.66525400 \\ \mathrm{~N} & 4.31645600 & 1.52911800 & 5.48560300 \\ \mathrm{C} & 5.01869700 & 1.10143600 & 4.25735100 \\ \mathrm{C} & 6.52290800 & 0.99675200 & 4.50308500 \\ \mathrm{O} & 7.25081800 & 0.15817700 & 3.93164800 \\ \mathrm{C} & 4.71790000 & 2.08742100 & 3.08491900 \\ \mathrm{C} & 5.60814900 & 3.34126000 & 3.04075400 \\ \mathrm{~S} & 5.06226100 & 4.58258400 & 1.76574700 \\ \mathrm{C} & 5.39406200 & 3.62824300 & 0.21652400 \\ \mathrm{~N} & 5.63503000 & -2.35689300 & 3.23083900 \\ \mathrm{C} & 4.87384700 & -2.52231400 & 1.98832900 \\ \mathrm{C} & 5.33950900 & -1.44777200 & 0.96594400 \\ \mathrm{O} & 5.10854900 & -0.21979500 & 1.14592700 \\ \mathrm{C} & 3.34109600 & -2.41144300 & 2.18504100 \\ \mathrm{~S} & 2.38649000 & -3.04748400 & 0.70130000 \\ \mathrm{~N} & 6.03338300 & -1.87883200 & -0.14023300 \\ \mathrm{C} & 6.43807500 & -1.00499300 & -1.27619300 \\ \mathrm{C} & 7.42924000 & 0.08477000 & -0.83326600 \\ \mathrm{O} & 8.48240200 & 0.32796100 & -1.45420200 \\ \mathrm{C} & 5.21080000 & -0.38093000 & -2.03871600 \\ & & & \mathrm{~S} 80 \\ \mathrm{C} & & & \end{array}$




\begin{tabular}{|c|c|c|c|}
\hline $\mathrm{C}$ & 4.32472700 & -1.52309500 & -2.60180000 \\
\hline $\mathrm{C}$ & 5.69201600 & 0.57907400 & -3.15164200 \\
\hline $\mathrm{C}$ & 3.02686500 & -1.03925100 & -3.28076400 \\
\hline $\mathrm{N}$ & -9.01794000 & -4.76396600 & -3.94557800 \\
\hline $\mathrm{C}$ & -8.63723000 & -3.87771700 & -5.07543300 \\
\hline $\mathrm{C}$ & -9.20637000 & -4.48446600 & -6.36817400 \\
\hline $\mathrm{O}$ & -8.75361800 & -4.30511600 & -7.51290600 \\
\hline $\mathrm{C}$ & -7.13225100 & -3.54613200 & -5.25201300 \\
\hline $\mathrm{C}$ & -6.47447700 & -2.66360900 & -4.21475000 \\
\hline $\mathrm{C}$ & -6.98629300 & -1.64483800 & -3.41623700 \\
\hline $\mathrm{C}$ & -5.04582400 & -2.69391100 & -3.96442100 \\
\hline $\mathrm{N}$ & -5.95623100 & -1.04826700 & -2.67449500 \\
\hline $\mathrm{C}$ & -4.75450200 & -1.67995100 & -2.99832900 \\
\hline $\mathrm{C}$ & -3.99350900 & -3.49502900 & -4.47155600 \\
\hline $\mathrm{C}$ & -3.44879300 & -1.47146700 & $-2.5095580 \mathrm{C}$ \\
\hline $\mathrm{C}$ & -2.69334600 & -3.28380500 & -3.99508000 \\
\hline $\mathrm{C}$ & -2.42869700 & -2.28735300 & -3.01543800 \\
\hline $\mathrm{N}$ & 3.25760000 & -5.23732200 & -8.05012100 \\
\hline $\mathrm{C}$ & 3.91333100 & -5.90772800 & -6.91900400 \\
\hline $\mathrm{C}$ & 2.94123400 & -6.68830100 & -6.02179800 \\
\hline $\mathrm{O}$ & 1.75644200 & -6.91234800 & -6.33832700 \\
\hline $\mathrm{C}$ & 4.76969700 & -4.88901900 & -6.10392100 \\
\hline $\mathrm{C}$ & 5.70233300 & -5.50791800 & -5.09000500 \\
\hline $\mathrm{C}$ & 6.95051800 & -6.05263400 & -5.35279200 \\
\hline $\mathrm{C}$ & 5.48872900 & -5.63599100 & -3.65632400 \\
\hline $\mathrm{N}$ & 7.52539300 & -6.51865600 & -4.16490200 \\
\hline $\mathrm{C}$ & 6.64883000 & -6.26795200 & -3.10496100 \\
\hline $\mathrm{C}$ & 4.43782400 & -5.25450100 & -2.78593900 \\
\hline $\mathrm{C}$ & 6.78367500 & -6.52535000 & -1.72698800 \\
\hline $\mathrm{C}$ & 4.56245800 & -5.50304200 & -1.41221800 \\
\hline $\mathrm{C}$ & 5.72659300 & -6.13387300 & -0.88992300 \\
\hline $\mathrm{N}$ & 2.55602700 & 8.87944400 & -4.34881300 \\
\hline $\mathrm{C}$ & 3.57550700 & 7.82146200 & -4.46199400 \\
\hline $\mathrm{C}$ & 4.61662200 & 8.46079400 & -5.38949300 \\
\hline $\mathrm{O}$ & 4.98887800 & 8.01253700 & -6.49058600 \\
\hline $\mathrm{C}$ & 3.11174900 & 6.40054400 & -4.92573300 \\
\hline $\mathrm{C}$ & 1.74870100 & 5.94159400 & -4.44900200 \\
\hline $\mathrm{C}$ & 1.40715000 & 5.24599100 & -3.28851100 \\
\hline $\mathrm{C}$ & 0.52013100 & 6.06494300 & -5.21302600 \\
\hline $\mathrm{N}$ & 0.04967100 & 4.92870500 & -3.29093500 \\
\hline $\mathrm{C}$ & -0.51810700 & 5.41825900 & -4.46216600 \\
\hline $\mathrm{C}$ & 0.20490500 & 6.63976700 & -6.46975100 \\
\hline $\mathrm{C}$ & -1.84089300 & 5.32413500 & -4.93940500 \\
\hline $\mathrm{C}$ & -1.11288700 & 6.56011900 & -6.94189000 \\
\hline $\mathrm{C}$ & -2.12504300 & 5.90586800 & -6.18342600 \\
\hline $\mathrm{N}$ & -10.15158700 & -2.18786700 & 5.69161400 \\
\hline $\mathrm{C}$ & -8.88440900 & -1.77628000 & 5.12967600 \\
\hline $\mathrm{C}$ & -7.89667100 & -1.71747200 & 6.26809600 \\
\hline $\mathrm{O}$ & -8.15328600 & -2.12563900 & 7.41962800 \\
\hline $\mathrm{C}$ & -8.98285000 & -0.40645000 & 4.37652700 \\
\hline
\end{tabular}




\begin{tabular}{|c|c|c|c|}
\hline $\mathrm{C}$ & -7.66800300 & 0.15704800 & 3.84993300 \\
\hline $\mathrm{C}$ & -6.81145400 & -0.59985200 & 3.01549300 \\
\hline $\mathrm{C}$ & -7.27772400 & 1.47168200 & 4.19052800 \\
\hline $\mathrm{C}$ & -5.59810800 & -0.06254700 & 2.54466200 \\
\hline $\mathrm{C}$ & -6.07282900 & 2.01876500 & 3.70749200 \\
\hline $\mathrm{C}$ & -5.22427900 & 1.25374800 & 2.88433200 \\
\hline $\mathrm{C}$ & 0.23967400 & -0.64876300 & 6.08164800 \\
\hline $\mathrm{O}$ & 0.41002200 & -1.39758200 & 7.32038600 \\
\hline $\mathrm{C}$ & 0.21349300 & -1.53617800 & 4.85564000 \\
\hline $\mathrm{S}$ & -0.09093800 & -0.74856400 & 3.30610900 \\
\hline $\mathrm{C}$ & 0.43051400 & -2.88156500 & 4.90251300 \\
\hline $\mathrm{S}$ & 0.44071000 & -3.85764900 & 3.42636400 \\
\hline $\mathrm{C}$ & 0.72139700 & -3.58120600 & 6.22127900 \\
\hline $\mathrm{C}$ & 1.29149900 & -2.56094200 & 7.21577800 \\
\hline $\mathrm{C}$ & -2.84297500 & -5.82907500 & -0.29465900 \\
\hline $\mathrm{O}$ & -4.29726700 & -5.87880500 & -0.12011300 \\
\hline $\mathrm{C}$ & -2.24285100 & -4.52392600 & 0.20180600 \\
\hline $\mathrm{S}$ & -0.48833700 & -4.48478000 & 0.49066300 \\
\hline $\mathrm{C}$ & -3.01137000 & -3.42990100 & 0.45865100 \\
\hline $\mathrm{S}$ & -2.25938000 & -1.92561700 & 1.02766100 \\
\hline $\mathrm{C}$ & -4.52393600 & -3.45547300 & 0.29949800 \\
\hline $\mathrm{C}$ & -4.94774900 & -4.64798500 & -0.57463300 \\
\hline W & 0.03411100 & -2.41003300 & 1.54027000 \\
\hline $\mathrm{O}$ & -1.54287200 & 2.11385000 & 2.22725900 \\
\hline $\mathrm{O}$ & 1.66425300 & 2.11705600 & 4.52272700 \\
\hline $\mathrm{O}$ & 1.10895500 & 5.45570600 & 1.57627200 \\
\hline $\mathrm{O}$ & -0.07422700 & 3.76138900 & 3.31794800 \\
\hline $\mathrm{O}$ & 0.42505500 & -0.72675000 & 0.49333900 \\
\hline $\mathrm{H}$ & -6.90586900 & -1.29814500 & 6.02891500 \\
\hline $\mathrm{H}$ & -7.92019300 & 2.06850700 & 4.83660700 \\
\hline $\mathrm{H}$ & -5.79243400 & 3.03467500 & 3.97709800 \\
\hline $\mathrm{H}$ & -4.29012500 & 1.66920300 & 2.51652100 \\
\hline $\mathrm{H}$ & -4.93268300 & -0.66544000 & 1.93164000 \\
\hline $\mathrm{H}$ & -7.08617300 & -1.61429100 & 2.72914700 \\
\hline $\mathrm{H}$ & -9.69017800 & -0.56319300 & 3.54803400 \\
\hline $\mathrm{H}$ & -9.45802100 & 0.30588700 & 5.06130700 \\
\hline $\mathrm{H}$ & -10.10086300 & -2.57170600 & 6.62970700 \\
\hline $\mathrm{H}$ & -10.82778300 & -2.61104300 & 5.07008300 \\
\hline $\mathrm{H}$ & -8.45583300 & -2.51114500 & 4.41644400 \\
\hline $\mathrm{H}$ & 2.29925500 & -2.24844300 & 6.89559700 \\
\hline $\mathrm{H}$ & 1.34678200 & -2.96260200 & 8.23326800 \\
\hline $\mathrm{H}$ & -0.19801800 & -4.02569300 & 6.62972400 \\
\hline $\mathrm{H}$ & 1.43949200 & -4.39910700 & 6.06754400 \\
\hline $\mathrm{H}$ & -0.70533400 & -0.10563000 & 6.20108300 \\
\hline $\mathrm{H}$ & 1.04593800 & 0.09829700 & 5.99480100 \\
\hline $\mathrm{H}$ & -2.59940100 & -5.98212800 & -1.36132500 \\
\hline $\mathrm{H}$ & -2.45466400 & -6.68014400 & 0.27492100 \\
\hline $\mathrm{H}$ & -4.88008600 & -2.52357800 & -0.15916900 \\
\hline $\mathrm{H}$ & -4.99215700 & -3.53535000 & 1.29315300 \\
\hline $\mathrm{H}$ & -4.69024800 & -4.45703800 & -1.62723700 \\
\hline
\end{tabular}




\begin{tabular}{|c|c|c|c|}
\hline $\mathrm{I}$ & -6.02284800 & -4.84430500 & -0.49568200 \\
\hline & 3.36185600 & -7.09055200 & -5.08205000 \\
\hline & 7.67231100 & -7.00306300 & -1.31967300 \\
\hline & 5.79307400 & -6.31447500 & 0.18051000 \\
\hline & 3.77103700 & -5.19275300 & -0.73358700 \\
\hline & 3.54555700 & -4.76125300 & -3.16444500 \\
\hline & 8.44118900 & -6.93467400 & -4.08106400 \\
\hline & 7.47656900 & -6.13033700 & -6.29416600 \\
\hline & 4.09664000 & -4.17479300 & -5.60800600 \\
\hline & 5.34148200 & -4.31886400 & -6.84738300 \\
\hline & 2.94080900 & -4.29522100 & -7.82263800 \\
\hline & 2.48670000 & -5.78470400 & -8.43297500 \\
\hline & 4.61475200 & -6.66710100 & -7.30738900 \\
\hline & 4.98584900 & 9.43477700 & -5.01280600 \\
\hline & 2.02216100 & 8.84199900 & -3.48340200 \\
\hline & 1.93082000 & 8.92644300 & -5.15219400 \\
\hline $\mathrm{H}$ & 4.04756400 & 7.74428800 & -3.47031900 \\
\hline $\mathrm{H}$ & 3.12582600 & 6.38840100 & -6.02398700 \\
\hline $\mathrm{H}$ & 3.88627900 & 5.68557600 & -4.61384500 \\
\hline $\mathrm{H}$ & 2.03617900 & 4.94537500 & -2.46198000 \\
\hline $\mathrm{H}$ & -0.48298800 & 4.46352000 & -2.54696900 \\
\hline $\mathrm{H}$ & -2.60641400 & 4.79790300 & -4.37690400 \\
\hline $\mathrm{H}$ & -3.13487200 & 5.83978900 & -6.58276900 \\
\hline 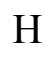 & -1.36892600 & 6.99084700 & -7.90843100 \\
\hline $\mathrm{H}$ & 0.97585400 & 7.11917000 & -7.07204800 \\
\hline H & 3.07162200 & -1.36967900 & 2.38968100 \\
\hline $\mathrm{H}$ & 3.05413700 & -3.02488500 & 3.04738900 \\
\hline $\mathrm{H}$ & 6.21421800 & -1.52723600 & 3.34828500 \\
\hline $\mathrm{H}$ & 5.17772900 & -2.68322700 & 4.07423900 \\
\hline U & 5.07538700 & -3.51651000 & 1.55105100 \\
\hline $\mathrm{H}$ & 6.15532400 & -2.87874300 & -0.25907900 \\
\hline $\mathrm{H}$ & 6.97678400 & -1.64640400 & -1.98737200 \\
\hline $\mathrm{H}$ & 4.61947100 & 0.18455600 & -1.30664600 \\
\hline $\mathrm{H}$ & 6.26048200 & 1.42845600 & -2.75439500 \\
\hline $\mathrm{H}$ & 6.33897900 & 0.05737100 & -3.87182500 \\
\hline $\mathrm{H}$ & 4.83542400 & 0.98696300 & -3.69936700 \\
\hline $\mathrm{H}$ & 3.21689700 & -0.45610400 & -4.19088900 \\
\hline $\mathrm{H}$ & 2.39731100 & -1.89583300 & -3.55720200 \\
\hline $\mathrm{H}$ & 2.44174600 & -0.41740100 & -2.59154300 \\
\hline $\mathrm{H}$ & 4.91973900 & -2.11558900 & -3.31795300 \\
\hline $\mathrm{H}$ & 4.04385000 & -2.19068000 & -1.77863100 \\
\hline $\mathrm{H}$ & 7.14327100 & 0.65255800 & 0.06617200 \\
\hline $\mathrm{H}$ & -3.72095700 & 1.26879500 & -2.85842500 \\
\hline $\mathrm{H}$ & -6.49644500 & 1.95394600 & -0.41198900 \\
\hline $\mathrm{H}$ & -4.89854900 & 1.48614100 & -0.21861000 \\
\hline $\mathrm{H}$ & -6.09477500 & 3.16009500 & -2.32292800 \\
\hline $\mathrm{H}$ & -4.14903000 & 4.60023900 & -2.05834900 \\
\hline $\mathrm{H}$ & -4.83418900 & 4.27157800 & -0.44048800 \\
\hline$n$ & -10.09908900 & -5.11880900 & -6.19161600 \\
\hline & -8.99712300 & -4.28697400 & -3.04639000 \\
\hline
\end{tabular}




\begin{tabular}{|c|c|c|c|}
\hline $\mathrm{H}$ & -8.45857300 & -5.61644900 & -3.90613000 \\
\hline $\mathrm{H}$ & -9.19562200 & -2.93603900 & -4.93427100 \\
\hline $\mathrm{H}$ & -6.58591900 & -4.50147500 & -5.29698200 \\
\hline $\mathrm{H}$ & -7.01405300 & -3.09743200 & -6.24941000 \\
\hline $\mathrm{H}$ & -8.00187800 & -1.28369300 & -3.32496400 \\
\hline $\mathrm{H}$ & -6.03294000 & -0.23866400 & -2.04553700 \\
\hline $\mathrm{H}$ & -3.23674600 & -0.74813600 & -1.72751500 \\
\hline $\mathrm{H}$ & -1.41987800 & -2.17807600 & -2.62660600 \\
\hline $\mathrm{H}$ & -1.87355700 & -3.89725200 & -4.36166000 \\
\hline $\mathrm{H}$ & -4.18650200 & -4.26500300 & -5.21587100 \\
\hline $\mathrm{H}$ & 6.96450000 & 1.78799100 & 5.13394800 \\
\hline $\mathrm{H}$ & 2.48184900 & -0.25205100 & 8.12941300 \\
\hline $\mathrm{H}$ & 4.12533700 & -0.58090500 & 8.74847000 \\
\hline $\mathrm{H}$ & 2.98992700 & 0.45475700 & 9.66624100 \\
\hline $\mathrm{H}$ & 1.98521700 & 2.03965100 & 7.22345600 \\
\hline $\mathrm{H}$ & 3.08665000 & 3.30525100 & 7.38078700 \\
\hline $\mathrm{H}$ & 4.61633600 & 1.89687500 & 8.61311800 \\
\hline $\mathrm{H}$ & 3.38475100 & 1.94790700 & 5.34382200 \\
\hline $\mathrm{H}$ & 4.66636000 & 0.10946700 & 3.94594000 \\
\hline $\mathrm{H}$ & 4.83477000 & 1.51347600 & 2.16709900 \\
\hline $\mathrm{H}$ & 3.66705700 & 2.38762200 & 3.16523900 \\
\hline $\mathrm{H}$ & 5.57284200 & 3.89767300 & 3.98806400 \\
\hline $\mathrm{H}$ & 6.65688500 & 3.09316900 & 2.83207300 \\
\hline $\mathrm{H}$ & 5.12029600 & 4.27908100 & -0.62159400 \\
\hline $\mathrm{H}$ & 6.45867400 & 3.37731800 & 0.14423100 \\
\hline $\mathrm{H}$ & 4.79424000 & 2.71473500 & 0.17797000 \\
\hline $\mathrm{H}$ & -2.78785200 & 6.51016200 & 0.96949500 \\
\hline $\mathrm{H}$ & -0.49027500 & 7.95071000 & 2.68364300 \\
\hline $\mathrm{H}$ & -0.00721800 & 7.03334100 & 1.33893800 \\
\hline $\mathrm{H}$ & -1.87273300 & 9.42118600 & 1.45202700 \\
\hline $\mathrm{H}$ & -0.49990600 & 9.36901200 & -0.53117100 \\
\hline $\mathrm{H}$ & -1.78952500 & 6.66963800 & -1.30950300 \\
\hline $\mathrm{H}$ & -0.68193800 & 7.62240100 & -2.29854900 \\
\hline $\mathrm{H}$ & -0.11574400 & 6.89271900 & -0.78949800 \\
\hline $\mathrm{H}$ & -3.38624700 & 10.67992800 & 0.31814600 \\
\hline $\mathrm{H}$ & -2.06198700 & 11.43090400 & -0.60203500 \\
\hline $\mathrm{H}$ & -3.67536400 & 11.26442200 & -1.32865900 \\
\hline $\mathrm{H}$ & -2.25400900 & 9.50878900 & -2.30582700 \\
\hline $\mathrm{H}$ & -3.42565800 & 8.73299000 & -1.24959200 \\
\hline $\mathrm{H}$ & -2.06576100 & 2.23548400 & 1.34370000 \\
\hline $\mathrm{H}$ & -1.41433100 & 1.18360300 & 2.50272800 \\
\hline $\mathrm{H}$ & -0.58746800 & 4.32912500 & 3.92347000 \\
\hline $\mathrm{H}$ & -0.72027700 & 3.06231400 & 2.81680700 \\
\hline $\mathrm{H}$ & 0.68473600 & 4.77231100 & 2.17295000 \\
\hline $\mathrm{H}$ & 2.07706200 & 5.32805200 & 1.53948700 \\
\hline $\mathrm{H}$ & 1.07542500 & 2.83238600 & 4.12408800 \\
\hline $\mathrm{H}$ & 1.41729900 & 1.26064100 & 4.10810000 \\
\hline $\mathrm{H}$ & -0.03701700 & 2.12721000 & -0.62707100 \\
\hline $\mathrm{H}$ & 1.49220100 & 1.64412500 & -1.36944800 \\
\hline $\mathrm{C}$ & 0.36857900 & 0.02087800 & -0.53403400 \\
\hline
\end{tabular}




$\begin{array}{lrcc}\mathrm{C} & 0.82382400 & 1.44780500 & -0.51766200 \\ \mathrm{H} & -0.08933100 & -0.38557800 & -1.44488200 \\ \mathrm{H} & 1.34233600 & 1.67662100 & 0.41757300\end{array}$

MoN-E , $E=-5352.083411$

$\begin{array}{lrrr}\mathrm{N} & 5.26350900 & 1.89005000 & -1.70802600 \\ \mathrm{C} & 4.38844500 & 3.06686600 & -2.00222800 \\ \mathrm{C} & 4.01157800 & 3.90579200 & -0.75691100 \\ \mathrm{O} & 3.21879400 & 4.86409400 & -0.82278400 \\ \mathrm{C} & 3.13894300 & 2.74544200 & -2.86773400 \\ \mathrm{C} & 2.34204200 & 1.48351600 & -2.44917200 \\ \mathrm{O} & 2.04638200 & 1.28587800 & -1.20920900 \\ \mathrm{O} & 2.03061900 & 0.71734400 & -3.44681800 \\ \mathrm{~N} & -0.90806700 & 2.58402900 & -7.49591100 \\ \mathrm{C} & 0.13266300 & 3.59466200 & -7.29021000 \\ \mathrm{C} & 1.54416600 & 2.97856200 & -7.37626600 \\ \mathrm{O} & 2.41485000 & 3.33758900 & -8.20087800 \\ \mathrm{C} & -0.09574400 & 4.36319700 & -5.95170500 \\ \mathrm{C} & 0.94778100 & 5.48995600 & -5.72234600 \\ \mathrm{C} & -0.15989500 & 3.42235900 & -4.72372000 \\ \mathrm{C} & 1.02208700 & 6.55440400 & -6.83964600 \\ \mathrm{~N} & -3.41227300 & -5.61069700 & -5.56553700 \\ \mathrm{C} & -4.30058700 & -6.39518300 & -4.69564200 \\ \mathrm{C} & -5.07006500 & -5.61146300 & -3.60623800 \\ \mathrm{O} & -6.00131900 & -6.18207600 & -2.95212100 \\ \mathrm{C} & -3.53773200 & -7.59003800 & -4.08759400 \\ \mathrm{~N} & -4.75406000 & -4.29916100 & -3.41869900 \\ \mathrm{C} & -5.35626500 & -3.52761000 & -2.31041400 \\ \mathrm{C} & -6.82821300 & -3.90406300 & -2.12691000 \\ \mathrm{O} & -7.37402600 & -4.03569100 & -1.01437500 \\ \mathrm{C} & -5.16053700 & -1.99793400 & -2.56616400 \\ \mathrm{C} & -6.20723100 & -1.33423400 & -3.48057700 \\ \mathrm{~S} & -5.72712200 & 0.36933900 & -4.05289400 \\ \mathrm{C} & -5.65864200 & 1.25976200 & -2.43488300 \\ \mathrm{~N} & -5.32829300 & -4.54247100 & 1.40387100 \\ \mathrm{C} & -4.50411100 & -3.42613100 & 1.88146200 \\ \mathrm{C} & -5.26489300 & -2.10880400 & 1.57696100 \\ \mathrm{O} & -5.59012000 & -1.81997500 & 0.39070200 \\ \mathrm{C} & -3.08973000 & -3.33731300 & 1.19669100 \\ \mathrm{~S} & -1.97453100 & -1.94451200 & 1.82135200 \\ \mathrm{~N} & -5.57024500 & -1.25848500 & 2.60956600 \\ \mathrm{C} & -6.10803300 & 0.11860100 & 2.41542500 \\ \mathrm{C} & -7.38416200 & 0.16327700 & 1.54940700 \\ \mathrm{O} & -8.45588900 & 0.63869700 & 1.97413700 \\ \mathrm{C} & -5.03278700 & 1.10649400 & 1.83136900 \\ \mathrm{C} & -3.85444800 & 1.25276900 & 2.82869800 \\ \mathrm{C} & -5.67344000 & 2.47524300 & 1.49745100 \\ \mathrm{C} & -2.66943800 & 2.07590000 & 2.28179900 \\ \mathrm{~N} & 9.70030700 & 1.38391300 & 4.76488600 \\ & & & \\ \mathrm{~N} & & & \end{array}$




\begin{tabular}{|c|c|c|c|}
\hline $\mathrm{C}$ & 9.19238700 & 2.77545400 & 4.68495400 \\
\hline $\mathrm{C}$ & 9.89652600 & 3.60716600 & 5.76555700 \\
\hline $\mathrm{O}$ & 9.44808300 & 4.64439300 & 6.28651700 \\
\hline $\mathrm{C}$ & 7.65529200 & 2.96786400 & 4.74335600 \\
\hline $\mathrm{C}$ & 6.87288200 & 2.51343700 & 3.53232400 \\
\hline $\mathrm{C}$ & 7.25463200 & 2.42458400 & 2.19786500 \\
\hline $\mathrm{C}$ & 5.46246200 & 2.17282800 & 3.57593900 \\
\hline $\mathrm{N}$ & 6.16024600 & 2.04357400 & 1.40943600 \\
\hline $\mathrm{C}$ & 5.04400200 & 1.88537200 & 2.23813900 \\
\hline $\mathrm{C}$ & 4.52100700 & 2.08637900 & 4.63095200 \\
\hline $\mathrm{C}$ & 3.71855700 & 1.51420100 & 1.92852000 \\
\hline $\mathrm{C}$ & 3.20591400 & 1.70799100 & 4.33053000 \\
\hline $\mathrm{C}$ & 2.81276000 & 1.42168100 & 2.99377800 \\
\hline $\mathrm{N}$ & -2.24725100 & 3.83010400 & 9.40142100 \\
\hline $\mathrm{C}$ & -2.80026600 & 2.47938200 & 9.22609300 \\
\hline $\mathrm{C}$ & -1.73504600 & 1.37374900 & 9.26698100 \\
\hline $\mathrm{O}$ & -0.55354000 & 1.58346600 & 9.60629400 \\
\hline $\mathrm{C}$ & -3.67066700 & 2.41493000 & 7.93214600 \\
\hline $\mathrm{C}$ & -4.50479800 & 1.16578700 & 7.78886900 \\
\hline $\mathrm{C}$ & -5.76446400 & 0.95615700 & 8.33071600 \\
\hline $\mathrm{C}$ & -4.16095100 & -0.04434400 & 7.05942800 \\
\hline $\mathrm{N}$ & -6.22211200 & -0.32030000 & 7.98775100 \\
\hline $\mathrm{C}$ & -5.25600400 & -0.95481500 & 7.20148500 \\
\hline $\mathrm{C}$ & -3.04241000 & -0.43153400 & 6.27955300 \\
\hline $\mathrm{C}$ & -5.25787100 & -2.22943600 & 6.60241500 \\
\hline $\mathrm{C}$ & -3.03735100 & -1.69391600 & 5.67234200 \\
\hline $\mathrm{C}$ & -4.13451800 & -2.58578800 & 5.83959900 \\
\hline $\mathrm{N}$ & -3.92780100 & 8.03700200 & -4.73640300 \\
\hline $\mathrm{C}$ & -4.50205400 & 7.91199000 & -3.38736000 \\
\hline $\mathrm{C}$ & -5.86821400 & 8.57255100 & -3.53125500 \\
\hline $\mathrm{O}$ & -6.28413600 & 9.53497800 & -2.85780600 \\
\hline $\mathrm{C}$ & -3.66224100 & 8.48030000 & -2.21954800 \\
\hline $\mathrm{C}$ & -2.35600900 & 7.75679100 & -1.97593600 \\
\hline $\mathrm{C}$ & -2.09103500 & 6.39749300 & -2.08947100 \\
\hline $\mathrm{C}$ & -1.12836400 & 8.37182500 & -1.50128600 \\
\hline $\mathrm{N}$ & -0.76922900 & 6.13723100 & -1.71382700 \\
\hline $\mathrm{C}$ & -0.15400700 & 7.33252300 & -1.34731700 \\
\hline $\mathrm{C}$ & -0.76165000 & 9.70404100 & -1.18837800 \\
\hline $\mathrm{C}$ & 1.15848700 & 7.57661500 & -0.89676200 \\
\hline $\mathrm{C}$ & 0.54211800 & 9.95900000 & -0.74096600 \\
\hline $\mathrm{C}$ & 1.48985400 & 8.90604400 & -0.59666700 \\
\hline $\mathrm{N}$ & 10.17697000 & -5.42613700 & -2.59669400 \\
\hline $\mathrm{C}$ & 8.87661400 & -4.79743400 & -2.50590900 \\
\hline $\mathrm{C}$ & 7.86288600 & -5.82191400 & -2.95244600 \\
\hline $\mathrm{O}$ & 8.15010300 & -7.01634300 & -3.17469400 \\
\hline $\mathrm{C}$ & 8.79548100 & -3.48431800 & -3.35368900 \\
\hline $\mathrm{C}$ & 7.42739000 & -2.81511200 & -3.43610000 \\
\hline $\mathrm{C}$ & 6.63404200 & -2.57501700 & -2.28915200 \\
\hline $\mathrm{C}$ & 6.92338100 & -2.40874700 & -4.69241100 \\
\hline 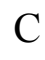 & 5.37060500 & -1.96149300 & -2.39571900 \\
\hline
\end{tabular}




\begin{tabular}{|c|c|c|c|}
\hline $\mathrm{C}$ & 5.66931400 & -1.77684600 & -4.80167000 \\
\hline $\mathrm{C}$ & 4.88308900 & -1.55380600 & -3.65465800 \\
\hline $\mathrm{C}$ & -0.25881900 & -5.65200300 & -2.61582400 \\
\hline $\mathrm{O}$ & -0.40715000 & -7.09819000 & -2.51759100 \\
\hline $\mathrm{C}$ & -0.07901300 & -4.98708800 & -1.26836300 \\
\hline $\mathrm{S}$ & 0.31917900 & -3.26930300 & -1.26323800 \\
\hline $\mathrm{C}$ & -0.17437400 & -5.66378700 & -0.08399600 \\
\hline $\mathrm{S}$ & 0.03487000 & -4.83592400 & 1.46270900 \\
\hline $\mathrm{C}$ & -0.47095000 & -7.15542500 & -0.05657700 \\
\hline $\mathrm{C}$ & -1.17490000 & -7.55489100 & -1.35875300 \\
\hline $\mathrm{C}$ & 3.70531000 & -2.51090500 & 4.60420700 \\
\hline $\mathrm{O}$ & 5.13709500 & -2.76557000 & 4.41712800 \\
\hline $\mathrm{C}$ & 2.97724000 & -2.35105000 & 3.28196500 \\
\hline $\mathrm{S}$ & 1.22136600 & -2.60695300 & 3.26853600 \\
\hline $\mathrm{C}$ & 3.65059600 & -2.10936500 & 2.11970100 \\
\hline $\mathrm{S}$ & 2.77847600 & -2.01015700 & 0.58189000 \\
\hline $\mathrm{C}$ & 5.16658800 & -1.97751700 & 2.07781400 \\
\hline $\mathrm{C}$ & 5.73726300 & -1.80074900 & 3.49545700 \\
\hline Mo & 0.47041600 & -2.52804400 & 1.01617500 \\
\hline $\mathrm{O}$ & 0.95040100 & -1.46597100 & -3.93054100 \\
\hline $\mathrm{O}$ & -2.24603600 & -3.15306800 & -3.91963600 \\
\hline $\mathrm{O}$ & -2.17053100 & 0.70925800 & -5.71222800 \\
\hline $\mathrm{O}$ & -0.87245400 & -1.61000900 & -5.51951700 \\
\hline $\mathrm{O}$ & 0.16921800 & -0.47105300 & 0.17809200 \\
\hline $\mathrm{H}$ & 6.82684900 & -5.46388600 & -3.06484100 \\
\hline $\mathrm{H}$ & 7.51465500 & -2.59215100 & -5.58876000 \\
\hline $\mathrm{H}$ & 5.29860800 & -1.47200300 & -5.77797500 \\
\hline $\mathrm{H}$ & 3.90683100 & -1.08393500 & -3.73925600 \\
\hline $\mathrm{H}$ & 4.75316700 & -1.82955600 & -1.50908800 \\
\hline $\mathrm{H}$ & 6.99104200 & -2.87175200 & -1.30401700 \\
\hline $\mathrm{H}$ & 9.53114200 & -2.79220900 & -2.91545700 \\
\hline $\mathrm{H}$ & 9.16027400 & -3.72749700 & -4.35875400 \\
\hline $\mathrm{H}$ & 10.15617100 & -6.43639700 & -2.69380300 \\
\hline $\mathrm{H}$ & 10.92109200 & -5.04865200 & -2.02491200 \\
\hline $\mathrm{H}$ & 8.58261600 & -4.53838000 & -1.46741200 \\
\hline $\mathrm{H}$ & -2.19061500 & -7.12743100 & -1.38016300 \\
\hline $\mathrm{H}$ & -1.24148900 & -8.64182500 & -1.47671700 \\
\hline $\mathrm{H}$ & 0.46402100 & -7.72475300 & 0.05208800 \\
\hline $\mathrm{H}$ & -1.10519800 & -7.40265000 & 0.80661300 \\
\hline $\mathrm{H}$ & 0.62003000 & -5.49609200 & -3.25283100 \\
\hline $\mathrm{H}$ & -1.13242900 & -5.23040400 & -3.13814300 \\
\hline $\mathrm{H}$ & 3.57852100 & -1.61055900 & 5.23206800 \\
\hline $\mathrm{H}$ & 3.32485600 & -3.37731200 & 5.15531000 \\
\hline $\mathrm{H}$ & 5.45631200 & -1.11624500 & 1.45897600 \\
\hline $\mathrm{H}$ & 5.59411700 & -2.87803300 & 1.60964000 \\
\hline $\mathrm{H}$ & 5.54868900 & -0.77724100 & 3.85524800 \\
\hline $\mathrm{H}$ & 6.81446500 & -2.00161800 & 3.52507600 \\
\hline $\mathrm{H}$ & -2.08054600 & 0.35315600 & 9.02316800 \\
\hline $\mathrm{H}$ & -6.09501900 & -2.91441200 & 6.71640100 \\
\hline $\mathrm{H}$ & -4.09581900 & -3.56293100 & 5.36484500 \\
\hline
\end{tabular}




\begin{tabular}{|c|c|c|c|}
\hline $\mathrm{H}$ & -2.20702400 & -1.99042100 & 5.03760300 \\
\hline $\mathrm{H}$ & -2.20186900 & 0.24238200 & 6.12986700 \\
\hline $\mathrm{H}$ & -7.12481600 & -0.70366300 & 8.22696200 \\
\hline $\mathrm{H}$ & -6.37206700 & 1.62578500 & 8.92335500 \\
\hline $\mathrm{H}$ & -3.01493000 & 2.53299000 & 7.05714800 \\
\hline $\mathrm{H}$ & -4.31727900 & 3.30115600 & 7.96439500 \\
\hline $\mathrm{H}$ & -1.97210800 & 4.26077900 & 8.51896000 \\
\hline & -1.46643200 & 3.84726500 & 10.05772700 \\
\hline & -3.47667900 & 2.25429200 & 10.06930400 \\
\hline $\mathrm{H}$ & -6.46824700 & 8.14818700 & -4.35929900 \\
\hline W & -3.27924600 & 7.30134400 & -5.00139000 \\
\hline & -3.55856200 & 8.96190200 & -4.94441900 \\
\hline & -4.69545600 & 6.84100300 & -3.22076200 \\
\hline 11 & -3.46421600 & 9.54232100 & -2.42299700 \\
\hline $\mathrm{H}$ & -4.28593800 & 8.47022300 & -1.31288000 \\
\hline $\mathrm{H}$ & -2.73727400 & 5.58808100 & -2.39924300 \\
\hline $\mathrm{H}$ & -0.32735700 & 5.22767000 & -1.70240400 \\
\hline $\mathrm{H}$ & 1.88018600 & 6.77164000 & -0.78339900 \\
\hline $\mathrm{H}$ & 2.49227700 & 9.13615300 & -0.24191400 \\
\hline $\mathrm{H}$ & 0.83922500 & 10.97652600 & -0.49387700 \\
\hline $\mathrm{H}$ & -1.47899500 & 10.51668600 & -1.28576300 \\
\hline $\mathrm{H}$ & -3.23306400 & -3.21710400 & 0.11651600 \\
\hline $\mathrm{H}$ & -2.56436100 & -4.28003500 & 1.37889700 \\
\hline $\mathrm{H}$ & -5.97447100 & -4.33242500 & 0.64101000 \\
\hline $\mathrm{H}$ & -4.83164600 & -5.42128000 & 1.29349700 \\
\hline $\mathrm{H}$ & -4.34322600 & -3.50892400 & 2.96602500 \\
\hline $\mathrm{H}$ & -5.25215000 & -1.50309800 & 3.54234500 \\
\hline $\mathrm{H}$ & -6.40101200 & 0.47953200 & 3.41102200 \\
\hline $\mathrm{H}$ & -4.64381500 & 0.65585500 & 0.90779900 \\
\hline $\mathrm{H}$ & -6.43941600 & 2.39919800 & 0.71530600 \\
\hline $\mathrm{H}$ & -6.15006700 & 2.91485700 & 2.38565900 \\
\hline $\mathrm{H}$ & -4.91152600 & 3.17736200 & 1.14092800 \\
\hline $\mathrm{H}$ & -2.92412800 & 3.13176900 & 2.12263400 \\
\hline $\mathrm{H}$ & -1.82143500 & 2.03873500 & 2.97768700 \\
\hline $\mathrm{H}$ & -2.32365100 & 1.65840100 & 1.32647600 \\
\hline $\mathrm{H}$ & -4.22739300 & 1.71153700 & 3.76074700 \\
\hline $\mathrm{H}$ & -3.47875800 & 0.25405500 & 3.07829900 \\
\hline $\mathrm{H}$ & -7.28452900 & -0.22494600 & 0.52569800 \\
\hline $\mathrm{H}$ & 4.52455700 & 3.65274200 & 0.18381600 \\
\hline $\mathrm{H}$ & 5.86125400 & 1.64408000 & -2.49541000 \\
\hline $\mathrm{H}$ & 4.71099400 & 1.07805600 & -1.42123100 \\
\hline $\mathrm{H}$ & 5.00405600 & 3.76634300 & -2.59402500 \\
\hline $\mathrm{H}$ & 2.46502200 & 3.61147500 & -2.83068600 \\
\hline $\mathrm{H}$ & 3.43887400 & 2.59631000 & -3.90978400 \\
\hline $\mathrm{H}$ & 10.88750800 & 3.19820100 & 6.05049200 \\
\hline $\mathrm{H}$ & 9.56165900 & 0.86410300 & 3.90056300 \\
\hline $\mathrm{H}$ & 9.31264200 & 0.86619200 & 5.55398800 \\
\hline $\mathrm{H}$ & 9.56426200 & 3.18857800 & 3.73115700 \\
\hline $\mathrm{H}$ & 7.28246000 & 2.43476900 & 5.63214100 \\
\hline 1 & 7.46112600 & 4.03315200 & 4.93722600 \\
\hline
\end{tabular}




\begin{tabular}{lrrr}
$\mathrm{H}$ & 8.21595300 & 2.61364100 & 1.73919200 \\
$\mathrm{H}$ & 6.14385800 & 1.95010800 & 0.39003500 \\
$\mathrm{H}$ & 3.39124500 & 1.27948700 & 0.91913000 \\
$\mathrm{H}$ & 1.79603400 & 1.09779000 & 2.79030100 \\
$\mathrm{H}$ & 2.47126400 & 1.61953800 & 5.12781000 \\
$\mathrm{H}$ & 4.81024100 & 2.30534400 & 5.65703600 \\
$\mathrm{H}$ & -7.42126100 & -3.96716400 & -3.05847400 \\
$\mathrm{H}$ & -2.61438400 & -7.26486100 & -3.59827600 \\
$\mathrm{H}$ & -4.16924100 & -8.11437000 & -3.36210600 \\
$\mathrm{H}$ & -3.25374700 & -8.27954600 & -4.89077500 \\
$\mathrm{H}$ & -2.59751500 & -5.21695100 & -5.09585200 \\
$\mathrm{H}$ & -3.88878500 & -4.90739500 & -6.12589300 \\
$\mathrm{H}$ & -5.10054000 & -6.81040200 & -5.32697900 \\
$\mathrm{H}$ & -3.88168300 & -3.90434800 & -3.81076400 \\
$\mathrm{H}$ & -4.84769300 & -3.76372900 & -1.36760200 \\
$\mathrm{H}$ & -5.17438200 & -1.52063100 & -1.58800800 \\
$\mathrm{H}$ & -4.16430700 & -1.86363100 & -3.00370100 \\
$\mathrm{H}$ & -6.33724900 & -1.89580200 & -4.41673500 \\
$\mathrm{H}$ & -7.18655700 & -1.26108200 & -2.99014500 \\
$\mathrm{H}$ & -5.45232900 & 2.31221200 & -2.66005000 \\
$\mathrm{H}$ & -6.62185900 & 1.18307800 & -1.91743600 \\
$\mathrm{H}$ & -4.86386800 & 0.86328800 & -1.79711100 \\
$\mathrm{H}$ & 1.72617900 & 2.12032300 & -6.69810300 \\
$\mathrm{H}$ & -0.94725100 & 2.15770100 & -8.41370100 \\
$\mathrm{H}$ & -1.28671100 & 2.00817600 & -6.73463500 \\
$\mathrm{H}$ & 0.08102700 & 4.32660600 & -8.10943300 \\
$\mathrm{H}$ & -1.08642300 & 4.83436700 & -6.06827700 \\
$\mathrm{H}$ & 0.77639200 & 2.87277700 & -4.57341200 \\
$\mathrm{H}$ & -0.34550300 & 4.01785800 & -3.81745900 \\
$\mathrm{H}$ & -0.95921000 & 2.67875900 & -4.80862000 \\
$\mathrm{H}$ & 1.43238500 & 6.13705000 & -7.76693200 \\
$\mathrm{H}$ & 0.02495100 & 6.96344800 & -7.06041200 \\
$\mathrm{H}$ & 1.66737000 & 7.38984500 & -6.53480100 \\
$\mathrm{H}$ & 0.69703100 & 5.98637700 & -4.77281800 \\
$\mathrm{H}$ & 1.94531500 & 5.04594300 & -5.58013500 \\
$\mathrm{H}$ & 1.37441700 & -0.55766900 & -3.65680600 \\
$\mathrm{H}$ & 0.96285500 & -2.13177600 & -3.20772300 \\
$\mathrm{H}$ & -0.61953500 & -1.93703600 & -6.40219800 \\
$\mathrm{H}$ & -0.00563200 & -1.52519500 & -4.83375500 \\
$\mathrm{H}$ & -1.68385400 & -0.16444400 & -5.61084500 \\
\hline & -3.05451900 & 0.67426300 & -5.29643300 \\
-1.79437400 & -2.56694800 & -4.61376400 \\
$\mathrm{H}$ & -1.78226800 & -3.06209900 & -3.05955300 \\
& -0.74513100 & -0.08767900 & -0.32226100 \\
& & & \\
$\mathrm{H}$ & & -12735600 & 0.22388500
\end{tabular}

MoN-ES , $E=-5429.376250$

$\begin{array}{llll}\mathrm{N} & -5.60685700 & 1.69965000 & -0.62091900 \\ \mathrm{C} & -5.09081900 & 2.79554600 & -1.47663800\end{array}$ 


\begin{tabular}{|c|c|c|c|}
\hline $\mathrm{C}$ & -4.38335200 & 2.32027200 & -2.77876500 \\
\hline $\mathrm{O}$ & -4.03778000 & 3.13848900 & -3.64874400 \\
\hline $\mathrm{C}$ & -4.16019100 & 3.83496000 & -0.74469200 \\
\hline $\mathrm{C}$ & -2.94977100 & 3.17524700 & -0.1153050 \\
\hline $\mathrm{O}$ & -1.85668700 & 3.15228100 & -0.9217470 \\
\hline $\mathrm{O}$ & -2.97326800 & 2.67192800 & 1.0310200 \\
\hline $\mathrm{N}$ & -0.29473300 & 7.58913400 & 2.40667800 \\
\hline $\mathrm{C}$ & -1.42095400 & 8.10866600 & 1.62762600 \\
\hline $\mathrm{C}$ & -2.73232600 & 7.37426900 & 1.9642470 \\
\hline $\mathrm{O}$ & -3.80147300 & 7.93967600 & 2.27932400 \\
\hline $\mathrm{C}$ & -1.10984200 & 8.08024200 & 0.0980460 \\
\hline $\mathrm{C}$ & -2.20324500 & 8.77819400 & -0.7542020 \\
\hline $\mathrm{C}$ & -0.84626700 & 6.65129900 & -0.4308420 \\
\hline $\mathrm{C}$ & -2.42667500 & 10.27272600 & -0.434107 \\
\hline $\mathrm{N}$ & 2.94675000 & 1.51717600 & 7.88122600 \\
\hline $\mathrm{C}$ & 4.03605200 & 0.54052500 & 8.02257800 \\
\hline $\mathrm{C}$ & 4.87425100 & 0.25713700 & 6.75183000 \\
\hline $\mathrm{O}$ & 5.93249200 & -0.44636600 & 6.83143200 \\
\hline $\mathrm{C}$ & 3.49949400 & -0.77247600 & 8.62988300 \\
\hline $\mathrm{N}$ & 4.47402700 & 0.82862200 & 5.58099900 \\
\hline $\mathrm{C}$ & 5.16052500 & 0.51443900 & 4.31012500 \\
\hline $\mathrm{C}$ & 6.66894000 & 0.39365000 & 4.51672500 \\
\hline $\mathrm{O}$ & 7.38597700 & -0.39836600 & 3.87068100 \\
\hline $\mathrm{C}$ & 4.82899400 & 1.60623800 & 3.24340300 \\
\hline $\mathrm{C}$ & 5.73305800 & 2.84957900 & 3.27776600 \\
\hline $\mathrm{S}$ & 5.06855500 & 4.27034000 & 2.26978700 \\
\hline $\mathrm{C}$ & 4.96846800 & 3.48167700 & 0.59876400 \\
\hline $\mathrm{N}$ & 5.67880600 & -2.84828200 & 2.96252300 \\
\hline $\mathrm{C}$ & 4.88236800 & -2.88569400 & 1.72501700 \\
\hline $\mathrm{C}$ & 5.33469200 & -1.71468400 & 0.80928600 \\
\hline $\mathrm{O}$ & 5.17347200 & -0.50915100 & 1.14801500 \\
\hline $\mathrm{C}$ & 3.36260200 & -2.80961600 & 1.97427800 \\
\hline $\mathrm{S}$ & 2.39844300 & -3.30399200 & 0.46135700 \\
\hline $\mathrm{N}$ & 5.95480100 & -2.02779500 & -0.3789560 \\
\hline $\mathrm{C}$ & 6.34600000 & -1.02925800 & -1.4119100 \\
\hline $\mathrm{C}$ & 7.44342100 & -0.08141600 & -0.89511600 \\
\hline $\mathrm{O}$ & 8.43442100 & 0.24377600 & -1.57870600 \\
\hline $\mathrm{C}$ & 5.12546200 & -0.21710400 & -1.9893480 \\
\hline $\mathrm{C}$ & 4.07235800 & -1.19798300 & -2.56980800 \\
\hline $\mathrm{C}$ & 5.60372700 & 0.80064100 & $-3.0513170 c$ \\
\hline $\mathrm{C}$ & 2.76602500 & -0.51355900 & -3.0268230 \\
\hline $\mathrm{N}$ & -9.12713400 & -4.37118800 & -4.1694810 \\
\hline $\mathrm{C}$ & -8.71830700 & -3.41799100 & -5.2317720 \\
\hline $\mathrm{C}$ & -9.34418900 & -3.87608100 & -6.5549340 \\
\hline $\mathrm{O}$ & -8.91819300 & -3.59757800 & -7.6904210 \\
\hline $\mathrm{C}$ & -7.20062700 & -3.15167600 & -5.4069800 \\
\hline $\mathrm{C}$ & -6.52431900 & -2.35241100 & -4.3183290 \\
\hline $\mathrm{C}$ & -7.05018000 & -1.43107300 & -3.4202750 \\
\hline $\mathrm{C}$ & -5.09284800 & -2.38703600 & -4.07805600 \\
\hline $\mathrm{N}$ & -6.02425500 & -0.89603000 & -2.6270190 \\
\hline
\end{tabular}




\begin{tabular}{|c|c|c|c|}
\hline $\mathrm{C}$ & -4.81197800 & -1.47752300 & -3.01144200 \\
\hline $\mathrm{C}$ & -4.03068600 & -3.12153700 & -4.65999200 \\
\hline $\mathrm{C}$ & -3.51170700 & -1.31386800 & -2.49223700 \\
\hline $\mathrm{C}$ & -2.73447000 & -2.95022700 & -4.15558800 \\
\hline $\mathrm{C}$ & -2.48057200 & -2.06448400 & -3.07223400 \\
\hline $\mathrm{N}$ & 3.07788800 & -4.69756400 & -8.50180400 \\
\hline $\mathrm{C}$ & 3.73988000 & -5.45636900 & -7.42975400 \\
\hline $\mathrm{C}$ & 2.76689000 & -6.29724500 & -6.58994700 \\
\hline $\mathrm{O}$ & 1.58768900 & -6.51059700 & -6.93408200 \\
\hline $\mathrm{C}$ & 4.61068000 & -4.50966500 & -6.54752300 \\
\hline $\mathrm{C}$ & 5.56192500 & -5.21448800 & -5.60987000 \\
\hline $\mathrm{C}$ & 6.77973300 & -5.78266500 & -5.95397800 \\
\hline $\mathrm{C}$ & 5.39784600 & -5.42014200 & -4.17890600 \\
\hline $\mathrm{N}$ & 7.38249100 & -6.33632000 & -4.81859700 \\
\hline $\mathrm{C}$ & 6.55485500 & -6.12169700 & -3.71189500 \\
\hline $\mathrm{C}$ & 4.39267900 & -5.05948800 & -3.24738100 \\
\hline $\mathrm{C}$ & 6.73065300 & -6.46890300 & -2.35849300 \\
\hline $\mathrm{C}$ & 4.55679100 & -5.39962500 & -1.89740600 \\
\hline $\mathrm{C}$ & 5.71754000 & -6.09823200 & -1.45999700 \\
\hline $\mathrm{N}$ & 2.67574000 & 9.06642200 & -3.54838500 \\
\hline $\mathrm{C}$ & 3.49829700 & 7.97382600 & -4.09348700 \\
\hline $\mathrm{C}$ & 4.71409000 & 8.70804900 & -4.65330000 \\
\hline $\mathrm{O}$ & 5.07781000 & 8.70268500 & -5.84511400 \\
\hline $\mathrm{C}$ & 2.82836600 & 7.02810100 & -5.12812200 \\
\hline $\mathrm{C}$ & 1.55266500 & 6.36745000 & -4.65659200 \\
\hline $\mathrm{C}$ & 1.42133600 & 5.29395300 & -3.78247700 \\
\hline $\mathrm{C}$ & 0.20809100 & 6.69812500 & -5.09663100 \\
\hline $\mathrm{N}$ & 0.07543300 & 4.94056300 & -3.65798600 \\
\hline $\mathrm{C}$ & -0.69281400 & 5.78165800 & -4.46053400 \\
\hline $\mathrm{C}$ & -0.31807800 & 7.66902800 & -5.98437900 \\
\hline $\mathrm{C}$ & -2.08145300 & 5.79397000 & -4.69936900 \\
\hline $\mathrm{C}$ & -1.70043800 & 7.70031300 & -6.21423600 \\
\hline $\mathrm{C}$ & -2.57133000 & 6.76934900 & -5.58047500 \\
\hline $\mathrm{N}$ & -10.08226600 & -2.63724300 & 5.67319000 \\
\hline $\mathrm{C}$ & -8.80779300 & -2.23614700 & 5.11980200 \\
\hline $\mathrm{C}$ & -7.81173500 & -2.25590600 & 6.25417700 \\
\hline $\mathrm{O}$ & -8.08349300 & -2.69409000 & 7.39145200 \\
\hline $\mathrm{C}$ & -8.87318900 & -0.83487700 & 4.41652200 \\
\hline $\mathrm{C}$ & -7.51847200 & -0.23026500 & 4.07469600 \\
\hline $\mathrm{C}$ & -6.60254200 & -0.89168200 & 3.22246500 \\
\hline $\mathrm{C}$ & -7.12501800 & 0.99133200 & 4.66531900 \\
\hline $\mathrm{C}$ & -5.31629700 & -0.36573000 & 2.99209100 \\
\hline $\mathrm{C}$ & -5.84668300 & 1.53076500 & 4.42466100 \\
\hline $\mathrm{C}$ & -4.93336700 & 0.84896200 & 3.59822500 \\
\hline $\mathrm{C}$ & 0.33681600 & -1.28396400 & 6.04247100 \\
\hline $\mathrm{O}$ & 0.51306200 & -2.16391300 & 7.20313500 \\
\hline $\mathrm{C}$ & 0.28416400 & -2.02260000 & 4.71733100 \\
\hline $\mathrm{S}$ & -0.06118800 & -1.07857600 & 3.24955000 \\
\hline $\mathrm{C}$ & 0.48547400 & -3.36966600 & 4.62020100 \\
\hline $\mathrm{S}$ & 0.38416100 & -4.21848900 & 3.06870200 \\
\hline
\end{tabular}




\begin{tabular}{|c|c|c|c|}
\hline $\mathrm{C}$ & 0.79911800 & -4.20621900 & 5.85377500 \\
\hline $\mathrm{C}$ & 1.38491700 & -3.31269600 & 6.95277300 \\
\hline $\mathrm{C}$ & -2.94407500 & -5.77065000 & -0.80942800 \\
\hline $\mathrm{O}$ & -4.37404700 & -5.89499200 & -0.52968200 \\
\hline $\mathrm{C}$ & -2.34111000 & -4.52787300 & -0.18570600 \\
\hline S & -0.58810100 & -4.58147500 & 0.09882700 \\
\hline $\mathrm{C}$ & -3.10086700 & -3.47686900 & 0.22823200 \\
\hline S & -2.34643200 & -2.03119700 & 0.96193200 \\
\hline $\mathrm{C}$ & -4.62067500 & -3.52372900 & 0.14925600 \\
\hline $\mathrm{C}$ & -5.08584000 & -4.64540600 & -0.79827800 \\
\hline Mo & -0.01667600 & -2.62247600 & 1.29960400 \\
\hline $\mathrm{O}$ & -1.53128500 & 1.80488800 & 3.19728000 \\
\hline $\mathrm{O}$ & 1.83406600 & 1.49951900 & 4.75703400 \\
\hline $\mathrm{O}$ & 1.28341300 & 5.11253300 & 2.45562400 \\
\hline $\mathrm{O}$ & 0.04713700 & 3.46934000 & 4.15808700 \\
\hline $\mathrm{O}$ & 0.58901400 & -1.05141300 & 0.13612000 \\
\hline $\mathrm{H}$ & -6.80338900 & -1.87767500 & 6.02628900 \\
\hline $\mathrm{H}$ & -7.81638900 & 1.51127400 & 5.32726300 \\
\hline $\mathrm{H}$ & -5.55634400 & 2.46863200 & 4.89369700 \\
\hline $\mathrm{H}$ & -3.93304400 & 1.24311600 & 3.44836500 \\
\hline $\mathrm{H}$ & -4.60136100 & -0.90697100 & 2.37374000 \\
\hline $\mathrm{H}$ & -6.88161500 & -1.83237900 & 2.74874600 \\
\hline $\mathrm{H}$ & -9.48607300 & -0.96577300 & 3.51220700 \\
\hline $\mathrm{H}$ & -9.43126500 & -0.17126800 & 5.08688600 \\
\hline $\mathrm{H}$ & -10.03338300 & -3.05019300 & 6.59935600 \\
\hline $\mathrm{H}$ & -10.76424700 & -3.03663700 & 5.04205500 \\
\hline $\mathrm{H}$ & -8.39990400 & -2.95248700 & 4.37591300 \\
\hline $\mathrm{H}$ & 2.39092100 & -2.96954600 & 6.65809400 \\
\hline $\mathrm{H}$ & 1.45106500 & -3.83052500 & 7.91646500 \\
\hline $\mathrm{H}$ & -0.11492700 & -4.69709800 & 6.22055100 \\
\hline $\mathrm{H}$ & 1.51324500 & -5.00158400 & 5.59622800 \\
\hline $\mathrm{H}$ & -0.60361800 & -0.75169000 & 6.23246000 \\
\hline $\mathrm{H}$ & 1.14926300 & -0.53955000 & 6.03471600 \\
\hline $\mathrm{H}$ & -2.78802800 & -5.77221200 & -1.90436300 \\
\hline $\mathrm{H}$ & -2.48694100 & -6.67437800 & -0.39293600 \\
\hline $\mathrm{H}$ & -5.02211000 & -2.56382500 & -0.20675100 \\
\hline $\mathrm{H}$ & -5.02796300 & -3.69400300 & 1.15959300 \\
\hline $\mathrm{H}$ & -4.92023800 & -4.34471600 & -1.84530900 \\
\hline $\mathrm{H}$ & -6.14747300 & -4.88074300 & -0.65443700 \\
\hline $\mathrm{H}$ & 3.17994100 & -6.75075100 & -5.67047100 \\
\hline $\mathrm{H}$ & 7.61688900 & -6.99863800 & -2.01517100 \\
\hline $\mathrm{H}$ & 5.81466500 & -6.34863000 & -0.40607000 \\
\hline $\mathrm{H}$ & 3.79770900 & -5.11279900 & -1.17194500 \\
\hline $\mathrm{H}$ & 3.50292000 & -4.51722100 & -3.55891300 \\
\hline $\mathrm{H}$ & 8.28508000 & -6.78734300 & -4.79505200 \\
\hline $\mathrm{H}$ & 7.26684800 & -5.82216900 & -6.91848200 \\
\hline $\mathrm{H}$ & 3.94840300 & -3.84225200 & -5.97780000 \\
\hline $\mathrm{H}$ & 5.16776000 & -3.87649300 & -7.25035700 \\
\hline $\mathrm{H}$ & 2.76870600 & -3.77483700 & -8.19664200 \\
\hline $\mathrm{H}$ & 2.29713400 & -5.21094700 & -8.91167100 \\
\hline
\end{tabular}




\begin{tabular}{|c|c|c|c|}
\hline $\mathrm{H}$ & 4.43150600 & -6.18799600 & -7.88308800 \\
\hline & 5.23666900 & 9.32547900 & -3.89709300 \\
\hline & 2.08480900 & 8.80475400 & -2.76424700 \\
\hline & 2.15933900 & 9.58891200 & -4.25267400 \\
\hline & 3.84869400 & 7.38405600 & -3.23297900 \\
\hline & 2.62955300 & 7.60550300 & -6.04056300 \\
\hline & 3.57261500 & 6.27213000 & -5.41564800 \\
\hline & 2.18433300 & 4.74092200 & -3.25258300 \\
\hline & -0.29066700 & 4.20538700 & -3.06464800 \\
\hline & -2.74507300 & 5.06191900 & -4.24675200 \\
\hline & -3.63745500 & 6.80513500 & -5.79428100 \\
\hline & -2.11887500 & 8.43803600 & -6.89634000 \\
\hline & 0.33926500 & 8.37247200 & -6.49241900 \\
\hline & 3.08409800 & -1.79898400 & 2.29353900 \\
\hline & 3.10029700 & -3.51006500 & 2.77709800 \\
\hline & 6.29132700 & -2.05108800 & 3.12496600 \\
\hline $\mathrm{H}$ & 5.20159300 & -3.18627100 & 3.79091400 \\
\hline $\mathrm{H}$ & 5.07600400 & -3.82915100 & 1.18496200 \\
\hline $\mathrm{H}$ & 6.00428500 & -3.00729800 & -0.63943500 \\
\hline $\mathrm{H}$ & 6.78415700 & -1.59645900 & -2.24495900 \\
\hline $\mathrm{H}$ & 4.66386500 & 0.32385200 & -1.15319200 \\
\hline $\mathrm{H}$ & 6.30272500 & 1.53923600 & -2.64200100 \\
\hline $\mathrm{H}$ & 6.11197500 & 0.29199400 & -3.88362200 \\
\hline $\mathrm{H}$ & 4.74710500 & 1.34690100 & -3.46217100 \\
\hline $\mathrm{H}$ & 2.91678300 & 0.14330900 & -3.89405400 \\
\hline 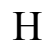 & 2.01660400 & -1.26686500 & -3.30164600 \\
\hline $\mathrm{H}$ & 2.33438600 & 0.08658900 & -2.21493900 \\
\hline $\mathrm{H}$ & 4.52335700 & -1.74364800 & -3.41704800 \\
\hline $\mathrm{H}$ & 3.81248100 & -1.94009900 & -1.80513600 \\
\hline 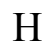 & 7.29097400 & 0.30973600 & 0.12408200 \\
\hline $\mathrm{H}$ & -4.21239400 & 1.23924500 & -2.89816600 \\
\hline $\mathrm{H}$ & -6.41472300 & 1.97280900 & -0.06525400 \\
\hline $\mathrm{H}$ & -4.90198600 & 1.26281900 & -0.02347200 \\
\hline $\mathrm{H}$ & -5.95897900 & 3.37374800 & -1.82361500 \\
\hline $\mathrm{H}$ & -3.83715300 & 4.58341300 & -1.47318300 \\
\hline $\mathrm{H}$ & -4.73411800 & 4.31317700 & 0.05520900 \\
\hline $\mathrm{H}$ & -10.25525200 & -4.49303200 & -6.41443700 \\
\hline $\mathrm{H}$ & -9.04772400 & -3.97623500 & -3.23437300 \\
\hline $\mathrm{H}$ & -8.62503400 & -5.25815600 & -4.21980800 \\
\hline $\mathrm{H}$ & -9.21980600 & -2.46172600 & -5.00213000 \\
\hline $\mathrm{H}$ & -6.69561100 & -4.12612900 & -5.50044400 \\
\hline $\mathrm{H}$ & -7.06289200 & -2.65496300 & -6.37871400 \\
\hline $\mathrm{H}$ & -8.07220900 & -1.10432100 & -3.28305300 \\
\hline $\mathrm{H}$ & -6.11909800 & -0.17025700 & -1.91505700 \\
\hline $\mathrm{H}$ & -3.30248200 & -0.70524900 & -1.61718200 \\
\hline $\mathrm{H}$ & -1.48257600 & -1.99631400 & -2.64880600 \\
\hline $\mathrm{H}$ & -1.90926600 & -3.52131700 & -4.57437600 \\
\hline $\mathrm{H}$ & -4.21514900 & -3.81648300 & -5.47692000 \\
\hline $\mathrm{H}$ & 7.12266200 & 1.13387000 & 5.19937600 \\
\hline & 2.64798000 & -1.15536000 & 8.05722700 \\
\hline
\end{tabular}




$\begin{array}{lrrr}\mathrm{H} & 4.29072500 & -1.52925800 & 8.65135300 \\ \mathrm{H} & 3.14805900 & -0.58030100 & 9.65008700 \\ \mathrm{H} & 2.14747500 & 1.18870500 & 7.34177800 \\ \mathrm{H} & 3.24270800 & 2.44383100 & 7.58362800 \\ \mathrm{H} & 4.76850200 & 0.95447600 & 8.73183500 \\ \mathrm{H} & 3.52810300 & 1.22888800 & 5.47956000 \\ \mathrm{H} & 4.80324500 & -0.44399500 & 3.91239600 \\ \mathrm{H} & 4.90150800 & 1.11925200 & 2.27321600 \\ \mathrm{H} & 3.78585900 & 1.90529800 & 3.39404700 \\ \mathrm{H} & 5.81479500 & 3.26721700 & 4.29166800 \\ \mathrm{H} & 6.74579100 & 2.62886400 & 2.91687700 \\ \mathrm{H} & 4.68847000 & 4.27228500 & -0.10676000 \\ \mathrm{H} & 5.94442500 & 3.07216800 & 0.31523000 \\ \mathrm{H} & 4.21261600 & 2.69256900 & 0.57857900 \\ \mathrm{H} & -2.65275700 & 6.26644400 & 1.98051100 \\ \mathrm{H} & -0.30001900 & 7.80326700 & 3.39686800 \\ \mathrm{H} & 0.18821100 & 6.71687200 & 2.17517700 \\ \mathrm{H} & -1.58267900 & 9.15748200 & 1.91140800 \\ \mathrm{H} & -0.17532700 & 8.65609100 & -0.00019900 \\ \mathrm{H} & -1.75631400 & 6.03686800 & -0.40368400 \\ \mathrm{H} & -0.51613200 & 6.68792400 & -1.47619400 \\ \mathrm{H} & -0.07144800 & 6.13354800 & 0.14530700 \\ \mathrm{H} & -2.87152100 & 10.41093300 & 0.55871500 \\ \mathrm{H} & -1.47820000 & 10.82841800 & -0.46515400 \\ \mathrm{H} & -3.10681100 & 10.72761500 & -1.16734900 \\ \mathrm{H} & -1.91503800 & 8.67661300 & -1.81101300 \\ \mathrm{H} & -3.15737400 & 8.24001900 & -0.64556700 \\ \mathrm{H} & -1.97884200 & 2.00160800 & 2.33849500 \\ \mathrm{H} & -1.23545700 & 0.85623000 & 3.27438000 \\ \mathrm{H} & -0.33315600 & 3.91487000 & 4.93765700 \\ \mathrm{H} & -0.64576300 & 2.78999600 & 3.73081300 \\ \mathrm{H} & 0.82901600 & 4.48106700 & 3.09651400 \\ \mathrm{H} & 2.25792500 & 5.01783200 & 2.50857900 \\ \mathrm{H} & 1.26404900 & 2.29945500 & 4.57811100 \\ \mathrm{H} & 1.52378800 & 0.75043700 & 4.19543200 \\ \mathrm{H} & -1.02958900 & 2.74682800 & -0.49519700 \\ \mathrm{H} & 1.48971400 & -1.28350900 & -0.20219300 \\ \mathrm{C} & 0.77813800 & 2.83933100 & 0.47190300 \\ \mathrm{H} & 0.58271000 & 1.64698600 & 0.22597900 \\ \mathrm{H} & 0.95669800 & 3.83912900 & 0.83031800 \\ & 0.47161200 & 0.51929900 & 0.12637200\end{array}$

MoN-TS , $E=-5429.353526$

$\begin{array}{llll}\mathrm{N} & 5.63198200 & 1.68461400 & 0.48170100 \\ \mathrm{C} & 5.19572900 & 2.83539900 & 1.31357300 \\ \mathrm{C} & 4.41469400 & 2.43719300 & 2.60060200 \\ \mathrm{O} & 4.18428000 & 3.27101100 & 3.49299500 \\ \mathrm{C} & 4.35775900 & 3.92820100 & 0.54323300 \\ \mathrm{C} & 3.09540200 & 3.32200800 & -0.03836100\end{array}$




\begin{tabular}{|c|c|c|c|}
\hline $\mathrm{O}$ & 1.98843600 & 3.52167100 & 0.70963400 \\
\hline $\mathrm{O}$ & 3.11318100 & 2.63689100 & -1.09301900 \\
\hline $\mathrm{N}$ & 0.41104100 & 7.48077100 & -2.86742800 \\
\hline $\mathrm{C}$ & 1.56223100 & 8.07165000 & -2.17573300 \\
\hline $\mathrm{C}$ & 2.84458700 & 7.25438900 & -2.40884500 \\
\hline $\mathrm{O}$ & 3.93024300 & 7.73396800 & -2.80090000 \\
\hline $\mathrm{C}$ & 1.25956300 & 8.26609100 & -0.65697100 \\
\hline $\mathrm{C}$ & 2.35921400 & 9.07845800 & 0.07785900 \\
\hline $\mathrm{C}$ & 0.99801700 & 6.92891700 & 0.07561000 \\
\hline $\mathrm{C}$ & 2.57014300 & 10.51656400 & -0.4451380 \\
\hline $\mathrm{N}$ & -2.90067200 & 1.15952200 & -8.01067400 \\
\hline $\mathrm{C}$ & -4.01473900 & 0.20629900 & -8.10872400 \\
\hline $\mathrm{C}$ & -4.86197000 & 0.00830400 & -6.82767300 \\
\hline $\mathrm{O}$ & -5.93382600 & -0.67768100 & -6.8746980 \\
\hline $\mathrm{C}$ & -3.50840600 & -1.14979300 & -8.6432180 \\
\hline $\mathrm{N}$ & -4.45680100 & 0.63190200 & -5.6846630 \\
\hline $\mathrm{C}$ & -5.16013600 & 0.39046400 & -4.4071180 \\
\hline $\mathrm{C}$ & -6.66859200 & 0.28710000 & -4.61691600 \\
\hline $\mathrm{O}$ & -7.40311300 & -0.44337100 & -3.9185590 \\
\hline $\mathrm{C}$ & -4.82487100 & 1.52015600 & -3.3829420 \\
\hline $\mathrm{C}$ & -5.68436300 & 2.78978200 & -3.4975780 \\
\hline $\mathrm{S}$ & -5.06380700 & 4.18521600 & -2.42995200 \\
\hline $\mathrm{C}$ & -5.32798800 & 3.45277000 & -0.75150700 \\
\hline $\mathrm{N}$ & -5.70467900 & -2.84967300 & -2.8843910 \\
\hline $\mathrm{C}$ & -4.92000700 & -2.86621700 & -1.6392180 \\
\hline $\mathrm{C}$ & -5.32167000 & -1.64050100 & -0.7709640 \\
\hline $\mathrm{O}$ & -5.07051700 & -0.45584100 & -1.1287090 \\
\hline $\mathrm{C}$ & -3.40152500 & -2.87398600 & -1.8896940 \\
\hline $\mathrm{S}$ & -2.46375400 & -3.32488000 & -0.34758100 \\
\hline $\mathrm{N}$ & -6.00645100 & -1.88787700 & 0.39797900 \\
\hline $\mathrm{C}$ & -6.38414600 & -0.84821300 & 1.39383500 \\
\hline $\mathrm{C}$ & -7.44336500 & 0.11092100 & 0.82199600 \\
\hline $\mathrm{O}$ & -8.42271400 & 0.51437600 & 1.47968300 \\
\hline $\mathrm{C}$ & -5.15137500 & -0.05670600 & 1.97466700 \\
\hline $\mathrm{C}$ & -4.12905800 & -1.05278700 & 2.5836590 \\
\hline $\mathrm{C}$ & -5.61865300 & 0.98766200 & 3.01519100 \\
\hline $\mathrm{C}$ & -2.80261600 & -0.39568200 & 3.02190300 \\
\hline $\mathrm{N}$ & 9.05791200 & -4.23881600 & 4.35674200 \\
\hline $\mathrm{C}$ & 8.65918700 & -3.22577300 & $5.3664950 \mathrm{c}$ \\
\hline $\mathrm{C}$ & 9.27885900 & -3.62031000 & 6.71284600 \\
\hline $\mathrm{O}$ & 8.85663900 & -3.27571300 & 7.83142300 \\
\hline $\mathrm{C}$ & 7.14534600 & -2.93656500 & 5.53255700 \\
\hline $\mathrm{C}$ & 6.46571500 & -2.18778500 & 4.4114610 \\
\hline $\mathrm{C}$ & 6.98537300 & -1.33003000 & 3.44914500 \\
\hline $\mathrm{C}$ & 5.02948100 & -2.21209900 & 4.20518100 \\
\hline $\mathrm{N}$ & 5.94983900 & -0.82515500 & 2.6480080 \\
\hline $\mathrm{C}$ & 4.73894700 & -1.36311100 & 3.0929280 \\
\hline $\mathrm{C}$ & 3.97107400 & -2.89446100 & 4.8531670 \\
\hline $\mathrm{C}$ & 3.43049800 & -1.21338000 & $2.5918180 \mathrm{C}$ \\
\hline $\mathrm{C}$ & 2.66665200 & -2.73427300 & 4.3676360 \\
\hline
\end{tabular}




\begin{tabular}{|c|c|c|c|}
\hline $\mathrm{C}$ & 2.40245300 & -1.91209900 & 3.23791100 \\
\hline $\mathrm{N}$ & -3.15437900 & -4.13935200 & 8.65884900 \\
\hline $\mathrm{C}$ & -3.82820000 & -4.96091500 & 7.64218200 \\
\hline $\mathrm{C}$ & -2.86643900 & -5.85953100 & 6.85050700 \\
\hline $\mathrm{O}$ & -1.68559900 & -6.05715000 & 7.1983690 \\
\hline $\mathrm{C}$ & -4.69963000 & -4.06736300 & 6.7064650 \\
\hline $\mathrm{C}$ & -5.65881900 & -4.82553000 & 5.8197490 \\
\hline $\mathrm{C}$ & -6.87863400 & -5.36239700 & 6.2044440 \\
\hline $\mathrm{C}$ & -5.50142400 & -5.12555200 & 4.4046550 \\
\hline $\mathrm{N}$ & -7.48911800 & -5.98547200 & 5.1097950 \\
\hline $\mathrm{C}$ & -6.66435300 & -5.84901000 & 3.9886000 \\
\hline $\mathrm{C}$ & -4.49758000 & -4.83322600 & 3.4480690 \\
\hline $\mathrm{C}$ & -6.84701500 & -6.28358600 & 2.6615620 \\
\hline $\mathrm{C}$ & -4.66876100 & -5.26008900 & 2.1239080 \\
\hline $\mathrm{C}$ & -5.83495200 & -5.97909900 & 1.73731500 \\
\hline $\mathrm{N}$ & -2.54581800 & 9.31694000 & 2.9938340 \\
\hline $\mathrm{C}$ & -3.34720300 & 8.28115400 & 3.6668000 \\
\hline $\mathrm{C}$ & -4.59075900 & 9.04776900 & 4.11185300 \\
\hline $\mathrm{O}$ & -4.97819100 & 9.17888400 & 5.28870000 \\
\hline $\mathrm{C}$ & -2.65473100 & 7.50574300 & 4.8145230 \\
\hline $\mathrm{C}$ & -1.40913800 & 6.74795000 & $4.4058660 \mathrm{C}$ \\
\hline $\mathrm{C}$ & -1.21627400 & 5.93346900 & 3.29528800 \\
\hline $\mathrm{C}$ & -0.17514400 & 6.68896400 & 5.16977100 \\
\hline $\mathrm{N}$ & 0.06517800 & 5.37449600 & $3.3288350 \mathrm{C}$ \\
\hline $\mathrm{C}$ & 0.72629500 & 5.81976700 & 4.47161500 \\
\hline $\mathrm{C}$ & 0.24879600 & 7.28426900 & 6.38351900 \\
\hline $\mathrm{C}$ & 2.01880100 & 5.52491900 & 4.95015400 \\
\hline $\mathrm{C}$ & 1.53609400 & 7.00441000 & 6.86192600 \\
\hline $\mathrm{C}$ & 2.40939700 & 6.13178900 & 6.15282300 \\
\hline $\mathrm{N}$ & 10.05217000 & -3.04681000 & -5.5623070 \\
\hline $\mathrm{C}$ & 8.78125400 & -2.60516000 & -5.03217900 \\
\hline $\mathrm{C}$ & 7.78830300 & -2.66401300 & -6.16778000 \\
\hline $\mathrm{O}$ & 8.05667700 & -3.15855800 & -7.28250900 \\
\hline $\mathrm{C}$ & 8.86301300 & -1.17436600 & -4.39352600 \\
\hline $\mathrm{C}$ & 7.51644400 & -0.53897800 & -4.0761420 \\
\hline $\mathrm{C}$ & 6.59719400 & -1.14706000 & -3.18849100 \\
\hline $\mathrm{C}$ & 7.13550200 & 0.65864200 & -4.72154100 \\
\hline $\mathrm{C}$ & 5.32024900 & -0.59208700 & -2.97472700 \\
\hline $\mathrm{C}$ & 5.86656300 & 1.22674500 & $-4.4984560 c$ \\
\hline $\mathrm{C}$ & 4.94971700 & 0.59827700 & -3.63443800 \\
\hline $\mathrm{C}$ & -0.28715100 & -1.58522000 & -6.0201940 \\
\hline $\mathrm{O}$ & -0.53290000 & -2.50158500 & -7.1384670 \\
\hline $\mathrm{C}$ & -0.25965300 & -2.26558800 & -4.6648300 \\
\hline S & 0.04727500 & -1.25294400 & -3.23826700 \\
\hline $\mathrm{C}$ & -0.48574800 & -3.60285500 & -4.50791300 \\
\hline $\mathrm{S}$ & -0.44325600 & -4.37101800 & $-2.9105460 c$ \\
\hline $\mathrm{C}$ & -0.80978800 & -4.48609800 & -5.7049000 \\
\hline $\mathrm{C}$ & -1.41179700 & -3.62830400 & -6.82351700 \\
\hline $\mathrm{C}$ & 2.85047700 & -5.75861200 & $1.0465350 \mathrm{C}$ \\
\hline $\mathrm{O}$ & 4.28811600 & -5.88534800 & 0.7930600 \\
\hline
\end{tabular}




\begin{tabular}{|c|c|c|c|}
\hline $\mathrm{C}$ & 2.24471400 & -4.52909400 & 0.39290900 \\
\hline S & 0.49057000 & -4.59521500 & 0.10320600 \\
\hline $\mathrm{C}$ & 3.00501400 & -3.48570200 & -0.03784600 \\
\hline $\mathrm{S}$ & 2.25131100 & -2.06280700 & -0.82269600 \\
\hline $\mathrm{C}$ & 4.52465100 & -3.52994200 & 0.04843600 \\
\hline $\mathrm{C}$ & 4.98759800 & -4.62195300 & 1.03169700 \\
\hline Mo & -0.06995400 & -2.69503400 & -1.22188700 \\
\hline $\mathrm{O}$ & 1.62202800 & 1.59694800 & -3.15469900 \\
\hline $\mathrm{O}$ & -1.80398400 & 1.27507400 & -4.82050200 \\
\hline $\mathrm{O}$ & -1.04114300 & 4.91534300 & -2.42656200 \\
\hline $\mathrm{O}$ & 0.02872100 & 3.20122300 & -4.18666000 \\
\hline $\mathrm{O}$ & -0.72198400 & -1.01433900 & -0.29142500 \\
\hline $\mathrm{H}$ & 6.78488300 & -2.26113200 & -5.96086700 \\
\hline $\mathrm{H}$ & 7.82972300 & 1.13757900 & -5.41087500 \\
\hline $\mathrm{H}$ & 5.58633000 & 2.14601800 & -5.00862200 \\
\hline $\mathrm{H}$ & 3.95664200 & 1.01447300 & -3.49528500 \\
\hline $\mathrm{H}$ & 4.60303600 & -1.09148900 & -2.32502500 \\
\hline $\mathrm{H}$ & 6.86715500 & -2.06783000 & -2.67223000 \\
\hline $\mathrm{H}$ & 9.47659500 & -1.27102100 & -3.48537000 \\
\hline $\mathrm{H}$ & 9.42699200 & -0.54796400 & -5.09408400 \\
\hline $\mathrm{H}$ & 10.00080600 & -3.50283700 & -6.46788100 \\
\hline $\mathrm{H}$ & 10.72824600 & -3.42309000 & -4.91094400 \\
\hline $\mathrm{H}$ & 8.36210400 & -3.28155400 & -4.25769800 \\
\hline $\mathrm{H}$ & -2.40692200 & -3.26329400 & -6.51821600 \\
\hline $\mathrm{H}$ & -1.50588600 & -4.18020500 & -7.76581400 \\
\hline $\mathrm{H}$ & 0.09927800 & -4.98769000 & -6.06976600 \\
\hline $\mathrm{H}$ & -1.51932300 & -5.27236200 & -5.40997100 \\
\hline $\mathrm{H}$ & 0.68005500 & -1.11829900 & -6.24616300 \\
\hline $\mathrm{H}$ & -1.05314000 & -0.79315100 & -6.03438800 \\
\hline $\mathrm{H}$ & 2.67682800 & -5.74208000 & 2.13844100 \\
\hline $\mathrm{H}$ & 2.40396000 & -6.67133400 & 0.63852700 \\
\hline $\mathrm{H}$ & 4.92642100 & -2.56033900 & 0.37579600 \\
\hline $\mathrm{H}$ & 4.93609900 & -3.73187000 & -0.95447700 \\
\hline $\mathrm{H}$ & 4.81008300 & -4.29233000 & 2.06812700 \\
\hline $\mathrm{H}$ & 6.05229600 & -4.85298500 & 0.90460100 \\
\hline $\mathrm{H}$ & -3.28941300 & -6.36886500 & 5.96545900 \\
\hline $\mathrm{H}$ & -7.73728900 & -6.83011000 & 2.35705600 \\
\hline $\mathrm{H}$ & -5.93712100 & -6.29812700 & 0.70257300 \\
\hline $\mathrm{H}$ & -3.91040200 & -5.02603100 & 1.37916700 \\
\hline $\mathrm{H}$ & -3.60329300 & -4.27759700 & 3.72041500 \\
\hline $\mathrm{H}$ & -8.39386000 & -6.43265300 & 5.11937400 \\
\hline $\mathrm{H}$ & -7.36213300 & -5.33579400 & 7.17120600 \\
\hline $\mathrm{H}$ & -4.03731000 & -3.44040300 & 6.09256100 \\
\hline $\mathrm{H}$ & -5.25024700 & -3.38850400 & 7.37066800 \\
\hline $\mathrm{H}$ & -2.84497000 & -3.23840500 & 8.29469000 \\
\hline $\mathrm{H}$ & -2.37226400 & -4.62913400 & 9.09412500 \\
\hline $\mathrm{H}$ & -4.52088600 & -5.65800200 & 8.14564000 \\
\hline $\mathrm{H}$ & -5.11110400 & 9.55357500 & 3.27563000 \\
\hline $\mathrm{H}$ & -1.93273400 & 8.97318100 & 2.26005200 \\
\hline $\mathrm{H}$ & -2.05677800 & 9.93839600 & 3.63444700 \\
\hline
\end{tabular}




$\begin{array}{lrrr}\mathrm{H} & -3.67650900 & 7.57744600 & 2.88700600 \\ \mathrm{H} & -2.40316900 & 8.22267800 & 5.60789200 \\ \mathrm{H} & -3.39612300 & 6.82623600 & 5.26131700 \\ \mathrm{H} & -1.89347800 & 5.69231500 & 2.48769900 \\ \mathrm{H} & 0.46282200 & 4.76218100 & 2.62547500 \\ \mathrm{H} & 2.68481800 & 4.84389900 & 4.42714000 \\ \mathrm{H} & 3.39762400 & 5.92128600 & 6.55599100 \\ \mathrm{H} & 1.87530300 & 7.45080900 & 7.79480900 \\ \mathrm{H} & -0.41471400 & 7.94102600 & 6.94291400 \\ \mathrm{H} & -3.07787100 & -1.89542400 & -2.26178800 \\ \mathrm{H} & -3.17180400 & -3.62730300 & -2.65382800 \\ \mathrm{H} & -6.34140600 & -2.07541200 & -3.06034500 \\ \mathrm{H} & -5.22038700 & -3.19251600 & -3.70614900 \\ \mathrm{H} & -5.15808600 & -3.77546200 & -1.05846300 \\ \mathrm{H} & -6.12623900 & -2.85716000 & 0.67268300 \\ \mathrm{H} & -6.85483000 & -1.37665200 & 2.23471400 \\ \mathrm{H} & -4.66589600 & 0.45959500 & 1.13674600 \\ \mathrm{H} & -6.30187900 & 1.73104500 & 2.58857000 \\ \mathrm{H} & -6.14111000 & 0.50209600 & 3.85248300 \\ \mathrm{H} & -4.75508100 & 1.52535800 & 3.42273700 \\ \mathrm{H} & -2.93488600 & 0.30540900 & 3.85678300 \\ \mathrm{H} & -2.08454000 & -1.16358900 & 3.33774000 \\ \mathrm{H} & -2.34128700 & 0.14921400 & 2.18777700 \\ \mathrm{H} & -4.59792400 & -1.56029900 & 3.44475200 \\ \mathrm{H} & -3.14408100 & -1.01984200 & -9.66868800 \\ \mathrm{H} & -3.89001800 & -1.82442100 & 1.84197900 \\ \mathrm{H} & -7.27611800 & 0.43344200 & -0.21925500 \\ \mathrm{H} & 4.07727600 & 1.39134700 & 2.68217500 \\ \mathrm{H} & 6.48918700 & 1.87142100 & -0.03462000 \\ \mathrm{H} & 4.91059000 & 1.33328400 & -0.15178400 \\ \mathrm{H} & 6.09864800 & 3.34853800 & 1.67197200 \\ \mathrm{H} & 4.10224500 & 4.73228800 & 1.23798500 \\ \mathrm{H} & 4.96714500 & 4.31840700 & -0.27840600 \\ \mathrm{H} & 10.18124800 & -4.25653500 & 6.60650900 \\ \mathrm{H} & 8.98702100 & -3.89326900 & 3.40167500 \\ \mathrm{H} & 8.54498100 & -5.11577100 & 4.45231000 \\ \mathrm{H} & 9.16970500 & -2.28801300 & 5.08615900 \\ \mathrm{H} & 6.63268700 & -3.90066500 & 5.67851600 \\ \mathrm{H} & 7.01704000 & -2.39024400 & 6.47860700 \\ \mathrm{H} & -1.00908800 & -1.02981000 & 3.27033600 \\ \mathrm{H} & 3.22063600 & -0.65838800 & 1.68188300 \\ \mathrm{H} & 2.105516000 & -10566100 & -7.75051000\end{array}$




\begin{tabular}{lrrr}
$\mathrm{H}$ & -4.73438900 & 0.60160200 & -8.84123700 \\
$\mathrm{H}$ & -3.50273200 & 1.01417800 & -5.59252800 \\
$\mathrm{H}$ & -4.82247200 & -0.55465900 & -3.96245200 \\
$\mathrm{H}$ & -4.93742800 & 1.07764700 & -2.39510100 \\
$\mathrm{H}$ & -3.76817800 & 1.77815100 & -3.51397000 \\
$\mathrm{H}$ & -5.66665900 & 3.20454000 & -4.51555800 \\
$\mathrm{H}$ & -6.73126700 & 2.60567100 & -3.22387200 \\
$\mathrm{H}$ & -5.01403100 & 4.20750600 & -0.02146400 \\
$\mathrm{H}$ & -6.38926300 & 3.22514700 & -0.59961200 \\
$\mathrm{H}$ & -4.73295300 & 2.54543900 & -0.61776100 \\
$\mathrm{H}$ & 2.72871800 & 6.15902800 & -2.26806500 \\
$\mathrm{H}$ & 0.42740600 & 7.54704600 & -3.87938100 \\
$\mathrm{H}$ & -0.03468000 & 6.62425900 & -2.51913200 \\
$\mathrm{H}$ & 1.75062200 & 9.06378900 & -2.60708400 \\
$\mathrm{H}$ & 0.32686600 & 8.85268100 & -0.64085300 \\
$\mathrm{H}$ & 1.91343900 & 6.32842900 & 0.16177700 \\
$\mathrm{H}$ & 0.64635100 & 7.12244700 & 1.09725100 \\
$\mathrm{H}$ & 0.24391800 & 6.31448600 & -0.43003700 \\
$\mathrm{H}$ & 2.99825500 & 10.52028700 & -1.45479800 \\
$\mathrm{H}$ & 1.61939200 & 11.06826600 & -0.47479500 \\
$\mathrm{H}$ & 3.26007700 & 11.06933100 & 0.20740500 \\
$\mathrm{H}$ & 2.08281200 & 9.12201300 & 1.14227300 \\
$\mathrm{H}$ & 3.31463600 & 8.53381100 & 0.03265000 \\
$\mathrm{H}$ & 2.07088100 & 1.84743400 & -2.30966300 \\
$\mathrm{H}$ & 1.32037000 & 0.64970200 & -3.18506300 \\
$\mathrm{H}$ & 0.40846500 & 3.64724300 & -4.96632700 \\
$\mathrm{H}$ & 0.72863900 & 2.54090100 & -3.74494000 \\
$\mathrm{H}$ & -0.64328100 & 4.25053700 & -3.06708000 \\
$\mathrm{H}$ & -2.01701700 & 4.84088100 & -2.41825100 \\
$\mathrm{H}$ & -1.21351200 & 2.05916800 & -4.63512400 \\
$\mathrm{H}$ & -1.51261200 & 0.52003500 & -4.25550400 \\
$\mathrm{H}$ & 1.14762300 & 2.97797200 & 0.38673900 \\
$\mathrm{H}$ & -1.65109700 & -1.11262900 & 0.02742500 \\
$\mathrm{C}$ & 0.22285000 & 0.99271500 & 0.15071600 \\
$\mathrm{C}$ & -0.25150300 & 2.11127700 & -0.12854300 \\
$\mathrm{H}$ & 0.88837600 & 0.21057300 & 0.46160200 \\
$\mathrm{H}$ & -0.95850200 & 2.78801200 & -0.56910100 \\
& & & \\
\hline & & & \\
& & &
\end{tabular}

MoN-EP1,$E=-5429.457104$

$\begin{array}{llll}\mathrm{N} & -5.65725500 & 1.51485800 & -0.91180100 \\ \mathrm{C} & -5.18710500 & 2.52927800 & -1.91752800 \\ \mathrm{C} & -4.40773400 & 1.91902600 & -3.10561100 \\ \mathrm{O} & -4.51576200 & 2.36640900 & -4.26228700 \\ \mathrm{C} & -4.26617900 & 3.65897100 & -1.32968300 \\ \mathrm{C} & -2.88344800 & 3.13820000 & -0.86611200 \\ \mathrm{O} & -1.82895800 & 3.55496600 & -1.45523500 \\ \mathrm{O} & -2.93259800 & 2.27262100 & 0.11692100 \\ \mathrm{~N} & -0.54583000 & 7.84339600 & 1.51563000 \\ \mathrm{C} & -1.71814000 & 8.37711600 & 0.80820800 \\ \mathrm{C} & -2.96864300 & 7.51721900 & 1.06038500 \\ & & & \mathrm{~S} 99\end{array}$




\begin{tabular}{|c|c|c|c|}
\hline $\mathrm{O}$ & -4.03616200 & 7.95213800 & 1.54727100 \\
\hline $\mathrm{C}$ & -1.42439700 & 8.55918800 & -0.71194200 \\
\hline $\mathrm{C}$ & -2.60469100 & 9.21550100 & -1.47760900 \\
\hline $\mathrm{C}$ & -1.00054100 & 7.24161900 & -1.39832700 \\
\hline $\mathrm{C}$ & -3.02392900 & 10.61101500 & -0.963790 \\
\hline $\mathrm{N}$ & 2.74565500 & 2.53403400 & 7.72369300 \\
\hline $\mathrm{C}$ & 3.84225400 & 1.58795600 & 7.96473100 \\
\hline $\mathrm{C}$ & 4.66293000 & 1.16297200 & 6.72328900 \\
\hline $\mathrm{O}$ & 5.70472800 & 0.44387900 & 6.86458500 \\
\hline $\mathrm{C}$ & 3.32365300 & 0.35237900 & 8.72963100 \\
\hline $\mathrm{N}$ & 4.27926900 & 1.62868100 & 5.49940100 \\
\hline $\mathrm{C}$ & 4.98969600 & 1.17691900 & 4.28502200 \\
\hline $\mathrm{C}$ & 6.49347400 & 1.09028200 & 4.53651700 \\
\hline $\mathrm{O}$ & 7.22984400 & 0.25599500 & 3.96822900 \\
\hline $\mathrm{C}$ & 4.69169400 & 2.13089800 & 3.08552800 \\
\hline $\mathrm{C}$ & 5.57494100 & 3.38945100 & 3.01729500 \\
\hline $\mathrm{S}$ & 5.03111300 & 4.59359200 & 1.70723500 \\
\hline $\mathrm{C}$ & 5.39400700 & 3.60686300 & 0.18578400 \\
\hline $\mathrm{N}$ & 5.68916400 & -2.29260800 & 3.32293100 \\
\hline $\mathrm{C}$ & 4.89073800 & -2.50449600 & 2.11093800 \\
\hline $\mathrm{C}$ & 5.29959500 & -1.44636000 & 1.04483300 \\
\hline $\mathrm{O}$ & 4.97388600 & -0.23092000 & 1.15560900 \\
\hline $\mathrm{C}$ & 3.36954400 & -2.43835500 & 2.34855300 \\
\hline $\mathrm{S}$ & 2.42801800 & -3.03862100 & 0.84844500 \\
\hline $\mathrm{N}$ & 6.04537100 & -1.88595200 & -0.02323900 \\
\hline $\mathrm{C}$ & 6.44793700 & -1.05021000 & -1.18843100 \\
\hline $\mathrm{C}$ & 7.43443200 & 0.05109400 & -0.76777900 \\
\hline $\mathrm{O}$ & 8.47836600 & 0.30339600 & -1.39939900 \\
\hline $\mathrm{C}$ & 5.23006100 & -0.44768600 & -1.98276500 \\
\hline $\mathrm{C}$ & 4.30663700 & -1.59528400 & -2.46946500 \\
\hline $\mathrm{C}$ & 5.72988200 & 0.42249900 & -3.15966600 \\
\hline $\mathrm{C}$ & 3.01139200 & -1.11208700 & -3.15679000 \\
\hline $\mathrm{N}$ & -8.96348400 & -5.00657600 & -3.8066760 \\
\hline $\mathrm{C}$ & -8.57805200 & -4.15050600 & -4.9583190 \\
\hline $\mathrm{C}$ & -9.14629000 & -4.78975600 & -6.2361100 \\
\hline $\mathrm{O}$ & -8.68651800 & -4.64872200 & -7.3833890 \\
\hline $\mathrm{C}$ & -7.07213300 & -3.82371200 & -5.1350530 \\
\hline $\mathrm{C}$ & -6.42682900 & -2.89960900 & -4.1258210 \\
\hline $\mathrm{C}$ & -6.95747000 & -1.87069200 & -3.3530450 \\
\hline $\mathrm{C}$ & -4.99640600 & -2.89241600 & -3.8807900 \\
\hline $\mathrm{N}$ & -5.93784500 & -1.23222300 & -2.6321630 \\
\hline $\mathrm{C}$ & -4.72361500 & -1.84624700 & -2.9441070 \\
\hline $\mathrm{C}$ & -3.92766500 & -3.68293600 & -4.3703530 \\
\hline $\mathrm{C}$ & -3.42045000 & -1.59429900 & -2.4686850 \\
\hline $\mathrm{C}$ & -2.63018700 & -3.42730500 & -3.9076280 \\
\hline $\mathrm{C}$ & -2.38258900 & -2.39743900 & -2.9581080 \\
\hline $\mathrm{N}$ & 3.32950500 & -5.49068100 & -7.8647160 \\
\hline $\mathrm{C}$ & 3.98603700 & -6.12096900 & -6.71102200 \\
\hline $\mathrm{C}$ & 3.01727800 & -6.88646100 & -5.79736400 \\
\hline $\mathrm{O}$ & 1.83830500 & -7.13637100 & -6.1155640 \\
\hline
\end{tabular}




\begin{tabular}{|c|c|c|c|}
\hline $\mathrm{C}$ & 4.82649300 & -5.06914100 & -5.92218400 \\
\hline $\mathrm{C}$ & 5.77134400 & -5.64825800 & -4.89619000 \\
\hline $\mathrm{C}$ & 7.02546400 & -6.18346600 & -5.15007700 \\
\hline $\mathrm{C}$ & 5.56554700 & -5.74134900 & -3.45888000 \\
\hline $\mathrm{N}$ & 7.61147900 & -6.61040600 & -3.95293400 \\
\hline $\mathrm{C}$ & 6.73613900 & -6.34359100 & -2.89618500 \\
\hline $\mathrm{C}$ & 4.51287300 & -5.35166200 & -2.59445100 \\
\hline $\mathrm{C}$ & 6.87946500 & -6.56403000 & -1.51269900 \\
\hline $\mathrm{C}$ & 4.64617600 & -5.56377300 & -1.21554100 \\
\hline $\mathrm{C}$ & 5.82023800 & -6.16564000 & -0.68163400 \\
\hline $\mathrm{N}$ & 2.50278700 & 8.71490700 & -4.51888800 \\
\hline $\mathrm{C}$ & 3.51280300 & 7.64823000 & -4.63334600 \\
\hline $\mathrm{C}$ & 4.57001700 & 8.28673700 & -5.54241300 \\
\hline $\mathrm{O}$ & 4.95072800 & 7.84565600 & -6.64363800 \\
\hline $\mathrm{C}$ & 3.03552300 & 6.23965700 & -5.11727300 \\
\hline $\mathrm{C}$ & 1.68017700 & 5.78029000 & -4.61955000 \\
\hline $\mathrm{C}$ & 1.36001500 & 5.07265100 & -3.46023100 \\
\hline $\mathrm{C}$ & 0.43546400 & 5.92139800 & -5.35351100 \\
\hline $\mathrm{N}$ & 0.00087400 & 4.76592500 & -3.43423700 \\
\hline $\mathrm{C}$ & -0.58986700 & 5.27305000 & -4.58614300 \\
\hline $\mathrm{C}$ & 0.09508900 & 6.51348300 & -6.59556800 \\
\hline $\mathrm{C}$ & -1.92395900 & 5.19417900 & -5.03369200 \\
\hline $\mathrm{C}$ & -1.23381400 & 6.44881600 & -7.03784600 \\
\hline $\mathrm{C}$ & -2.23284500 & 5.79271700 & -6.26371800 \\
\hline $\mathrm{N}$ & -10.14879300 & -2.19757500 & 5.75896700 \\
\hline $\mathrm{C}$ & -8.88377400 & -1.78797500 & 5.19099700 \\
\hline $\mathrm{C}$ & -7.89957000 & -1.69469000 & 6.33023800 \\
\hline $\mathrm{O}$ & -8.15779800 & -2.07538100 & 7.49098600 \\
\hline $\mathrm{C}$ & -8.98853900 & -0.43692100 & 4.40321200 \\
\hline $\mathrm{C}$ & -7.66517900 & 0.13253700 & 3.90689100 \\
\hline $\mathrm{C}$ & -6.80339000 & -0.61115700 & 3.06611000 \\
\hline $\mathrm{C}$ & -7.26251000 & 1.42902100 & 4.29825500 \\
\hline $\mathrm{C}$ & -5.56953600 & -0.08114800 & 2.64236500 \\
\hline $\mathrm{C}$ & -6.03617900 & 1.96874100 & 3.86356500 \\
\hline $\mathrm{C}$ & -5.17973100 & 1.21496400 & 3.03813400 \\
\hline $\mathrm{C}$ & 0.26473100 & -0.57187600 & 6.13146800 \\
\hline $\mathrm{O}$ & 0.40088100 & -1.28162600 & 7.39933400 \\
\hline $\mathrm{C}$ & 0.24460700 & -1.50048100 & 4.93528000 \\
\hline $\mathrm{S}$ & -0.13065100 & -0.79414500 & 3.35661700 \\
\hline $\mathrm{C}$ & 0.47910400 & -2.84169700 & 5.03841100 \\
\hline $\mathrm{S}$ & 0.46607300 & -3.88759900 & 3.61066100 \\
\hline $\mathrm{C}$ & 0.77518700 & -3.49084800 & 6.38216100 \\
\hline $\mathrm{C}$ & 1.30651100 & -2.42915000 & 7.35309800 \\
\hline $\mathrm{C}$ & -2.80311400 & -5.94517700 & -0.15266800 \\
\hline $\mathrm{O}$ & -4.24650200 & -5.98666300 & 0.06002100 \\
\hline $\mathrm{C}$ & -2.20415700 & -4.63783900 & 0.32816700 \\
\hline $\mathrm{S}$ & -0.48555700 & -4.63760600 & 0.73278200 \\
\hline $\mathrm{C}$ & -2.97116500 & -3.52662400 & 0.53181700 \\
\hline $\mathrm{S}$ & -2.23330200 & -2.02708800 & 1.11956800 \\
\hline $\mathrm{C}$ & -4.48104000 & -3.54746300 & 0.34578600 \\
\hline
\end{tabular}




\begin{tabular}{|c|c|c|c|}
\hline $\mathrm{C}$ & -4.90622900 & -4.78721600 & -0.45861100 \\
\hline Mo & 0.03362100 & -2.53969000 & 1.69533800 \\
\hline $\mathrm{O}$ & -1.73486100 & 2.08387900 & 2.35523600 \\
\hline $\mathrm{O}$ & 1.62186900 & 2.16095200 & 4.50120000 \\
\hline $\mathrm{O}$ & 1.00117600 & 5.40654400 & 1.56021400 \\
\hline $\mathrm{O}$ & -0.19608300 & 3.75475200 & 3.34040100 \\
\hline $\mathrm{O}$ & 0.66737600 & -0.88942600 & 0.32729500 \\
\hline $\mathrm{H}$ & -6.90991900 & -1.27781200 & 6.08360100 \\
\hline $\mathrm{H}$ & -7.91047300 & 2.01568600 & 4.94819200 \\
\hline $\mathrm{H}$ & -5.74442800 & 2.96983200 & 4.17373300 \\
\hline $\mathrm{H}$ & -4.22440800 & 1.62191000 & 2.71802000 \\
\hline $\mathrm{H}$ & -4.90023800 & -0.67605200 & 2.02515300 \\
\hline $\mathrm{H}$ & -7.08978100 & -1.61075400 & 2.74096100 \\
\hline $\mathrm{H}$ & -9.66888800 & -0.62435200 & 3.55894000 \\
\hline $\mathrm{H}$ & -9.49340600 & 0.28088900 & 5.06028000 \\
\hline $\mathrm{H}$ & -10.09767600 & -2.55616800 & 6.70698200 \\
\hline $\mathrm{H}$ & -10.81897300 & -2.64272200 & 5.14639600 \\
\hline $\mathrm{H}$ & -8.44561400 & -2.53514300 & 4.49636200 \\
\hline $\mathrm{H}$ & 2.31450300 & -2.10710900 & 7.04275000 \\
\hline $\mathrm{H}$ & 1.34911400 & -2.79702300 & 8.38388900 \\
\hline $\mathrm{H}$ & -0.13854100 & -3.94635300 & 6.79142200 \\
\hline $\mathrm{H}$ & 1.51447200 & -4.29549500 & 6.26165100 \\
\hline $\mathrm{H}$ & -0.67370300 & -0.01164000 & 6.21402800 \\
\hline $\mathrm{H}$ & 1.08467600 & 0.15908200 & 6.03472400 \\
\hline $\mathrm{H}$ & -2.58606600 & -6.10140100 & -1.22498500 \\
\hline $\mathrm{H}$ & -2.39774500 & -6.79143700 & 0.41141500 \\
\hline $\mathrm{H}$ & -4.82099900 & -2.64153800 & -0.17170300 \\
\hline $\mathrm{H}$ & -4.96164800 & -3.56205700 & 1.33678000 \\
\hline $\mathrm{H}$ & -4.65468900 & -4.65508700 & -1.52207400 \\
\hline $\mathrm{H}$ & -5.98025300 & -4.98159400 & -0.36311800 \\
\hline $\mathrm{H}$ & 3.43573000 & -7.25363600 & -4.84219300 \\
\hline $\mathrm{H}$ & 7.77540700 & -7.02041000 & -1.09704500 \\
\hline $\mathrm{H}$ & 5.89265100 & -6.31968100 & 0.39259700 \\
\hline $\mathrm{H}$ & 3.85242600 & -5.24777200 & -0.54281200 \\
\hline $\mathrm{H}$ & 3.61218800 & -4.88128400 & -2.98202600 \\
\hline $\mathrm{H}$ & 8.53222300 & -7.01402700 & -3.86276000 \\
\hline $\mathrm{H}$ & 7.54837700 & -6.27948900 & -6.09149500 \\
\hline $\mathrm{H}$ & 4.14285400 & -4.35399400 & -5.44240400 \\
\hline $\mathrm{H}$ & 5.38754600 & -4.50819900 & -6.68062900 \\
\hline $\mathrm{H}$ & 2.99223700 & -4.54973200 & -7.66298700 \\
\hline $\mathrm{H}$ & 2.57260900 & -6.06151200 & -8.24126200 \\
\hline $\mathrm{H}$ & 4.69781600 & -6.88298100 & -7.07442100 \\
\hline $\mathrm{H}$ & 4.94413900 & 9.25344600 & -5.15193000 \\
\hline $\mathrm{H}$ & 1.96056200 & 8.67465800 & -3.65883500 \\
\hline $\mathrm{H}$ & 1.88587300 & 8.77662300 & -5.32759600 \\
\hline $\mathrm{H}$ & 3.97322300 & 7.55383600 & -3.63764100 \\
\hline $\mathrm{H}$ & 3.02700200 & 6.24966300 & -6.21563600 \\
\hline $\mathrm{H}$ & 3.81292700 & 5.51518500 & -4.83578300 \\
\hline $\mathrm{H}$ & 2.00690100 & 4.75410800 & -2.65448200 \\
\hline $\mathrm{H}$ & -0.51705500 & 4.29713400 & -2.68138200 \\
\hline
\end{tabular}




\begin{tabular}{|c|c|c|c|}
\hline $\mathrm{H}$ & -2.68004800 & 4.66627200 & -4.45998300 \\
\hline $\mathrm{H}$ & -3.25211300 & 5.73863100 & -6.64029900 \\
\hline $\mathrm{H}$ & -1.50909400 & 6.89289400 & -7.99301100 \\
\hline $\mathrm{H}$ & 0.85524700 & 6.99463100 & -7.21014700 \\
\hline $\mathrm{H}$ & 3.07140700 & -1.41624000 & 2.60362200 \\
\hline $\mathrm{H}$ & 3.10781700 & -3.09784900 & 3.18335800 \\
\hline $\mathrm{H}$ & 6.24101600 & -1.44154500 & 3.41607400 \\
\hline $\mathrm{H}$ & 5.28410600 & -2.63820800 & 4.18511000 \\
\hline $\mathrm{H}$ & 5.10755200 & -3.50225200 & 1.68890000 \\
\hline $\mathrm{H}$ & 6.24393100 & -2.87908600 & -0.07771800 \\
\hline $\mathrm{H}$ & 6.98946800 & -1.71469600 & -1.87577100 \\
\hline $\mathrm{H}$ & 4.66034600 & 0.18269800 & -1.28764200 \\
\hline $\mathrm{H}$ & 6.33860200 & 1.27090900 & -2.82602400 \\
\hline $\mathrm{H}$ & 6.34194700 & -0.16943200 & -3.85564400 \\
\hline $\mathrm{H}$ & 4.87925500 & 0.82827400 & -3.71794200 \\
\hline $\mathrm{H}$ & 3.20536400 & -0.61118300 & -4.11372300 \\
\hline $\mathrm{H}$ & 2.34278600 & -1.96052500 & -3.35410600 \\
\hline $\mathrm{H}$ & 2.46032500 & -0.40855600 & -2.51862100 \\
\hline $\mathrm{H}$ & 4.87531200 & -2.23903200 & -3.16190800 \\
\hline $\mathrm{H}$ & 4.02687400 & -2.21665200 & -1.61057800 \\
\hline $\mathrm{H}$ & 7.15419500 & 0.61928400 & 0.13460600 \\
\hline $\mathrm{H}$ & -3.71085400 & 1.09698600 & -2.87005200 \\
\hline $\mathrm{H}$ & -6.49449100 & 1.83607900 & -0.42576500 \\
\hline $\mathrm{H}$ & -4.90551100 & 1.33268800 & -0.23516100 \\
\hline $\mathrm{H}$ & -6.07275400 & 3.01437200 & -2.34829800 \\
\hline $\mathrm{H}$ & -4.11991000 & 4.43096400 & -2.09301300 \\
\hline $\mathrm{H}$ & -4.77878900 & 4.10205500 & -0.46525700 \\
\hline $\mathrm{H}$ & -10.04597900 & -5.40999100 & -6.04516500 \\
\hline $\mathrm{H}$ & -8.94539200 & -4.50583300 & -2.92042000 \\
\hline $\mathrm{H}$ & -8.40400000 & -5.85749400 & -3.74281800 \\
\hline $\mathrm{H}$ & -9.13641900 & -3.20511500 & -4.84383500 \\
\hline $\mathrm{H}$ & -6.52336900 & -4.77891000 & -5.13914800 \\
\hline $\mathrm{H}$ & -6.94549700 & -3.41207800 & -6.14736100 \\
\hline $\mathrm{H}$ & -7.98018800 & -1.52875100 & -3.26735600 \\
\hline $\mathrm{H}$ & -6.02986900 & -0.40754000 & -2.02562700 \\
\hline $\mathrm{H}$ & -3.22038000 & -0.84310500 & -1.71021800 \\
\hline $\mathrm{H}$ & -1.37501000 & -2.24460900 & -2.58138400 \\
\hline $\mathrm{H}$ & -1.79813000 & -4.03077200 & -4.26331300 \\
\hline $\mathrm{H}$ & -4.10615500 & -4.47755800 & -5.09220100 \\
\hline $\mathrm{H}$ & 6.92738300 & 1.88991100 & 5.16114000 \\
\hline $\mathrm{H}$ & 2.46641600 & -0.09830100 & 8.21744700 \\
\hline $\mathrm{H}$ & 4.11966400 & -0.39314200 & 8.82792700 \\
\hline $\mathrm{H}$ & 2.98552100 & 0.66176000 & 9.72500200 \\
\hline $\mathrm{H}$ & 1.93612000 & 2.14916300 & 7.24126100 \\
\hline $\mathrm{H}$ & 3.02309700 & 3.43460600 & 7.34270800 \\
\hline $\mathrm{H}$ & 4.58154400 & 2.08696500 & 8.60910800 \\
\hline $\mathrm{H}$ & 3.34430500 & 2.03569800 & 5.34515300 \\
\hline $\mathrm{H}$ & 4.64616600 & 0.17445300 & 3.99753700 \\
\hline $\mathrm{H}$ & 4.81794700 & 1.53542400 & 2.18223300 \\
\hline $\mathrm{H}$ & 3.63787400 & 2.42505400 & 3.14838000 \\
\hline
\end{tabular}




$\begin{array}{lrrr}\mathrm{H} & 5.52673000 & 3.96862600 & 3.95021900 \\ \mathrm{H} & 6.62734100 & 3.14334800 & 2.82518700 \\ \mathrm{H} & 5.11949900 & 4.23269100 & -0.67086000 \\ \mathrm{H} & 6.46287300 & 3.36987500 & 0.12979600 \\ \mathrm{H} & 4.80740900 & 2.68407900 & 0.16343200 \\ \mathrm{H} & -2.84493200 & 6.43559200 & 0.84790800 \\ \mathrm{H} & -0.56140600 & 7.96074600 & 2.52351400 \\ \mathrm{H} & -0.08210400 & 6.97328500 & 1.23055100 \\ \mathrm{H} & -1.94441900 & 9.36696000 & 1.22758500 \\ \mathrm{H} & -0.56622500 & 9.25060700 & -0.74481700 \\ \mathrm{H} & -1.81165800 & 6.50265400 & -1.41882900 \\ \mathrm{H} & -0.71420000 & 7.43187600 & -2.44055900 \\ \mathrm{H} & -0.14517000 & 6.77355300 & -0.89955200 \\ \mathrm{H} & -3.47682000 & 10.55284400 & 0.03334600 \\ \mathrm{H} & -2.15730400 & 11.28612200 & -0.90777700 \\ \mathrm{H} & -3.76311000 & 11.06727500 & -1.63726100 \\ \mathrm{H} & -2.30931400 & 9.29514800 & -2.53472900 \\ \mathrm{H} & -3.47754800 & 8.54498900 & -1.45687400 \\ \mathrm{H} & -2.14297200 & 2.14542800 & 1.41222600 \\ \mathrm{H} & -1.54216300 & 1.16704000 & 2.64391800 \\ \mathrm{H} & -0.68318400 & 4.34793000 & 3.94297400 \\ \mathrm{H} & -0.86289400 & 3.05576100 & 2.88260500 \\ \mathrm{H} & 0.57175800 & 4.73055500 & 2.15989300 \\ \mathrm{H} & 1.96137500 & 5.24390600 & 1.48510900 \\ \mathrm{H} & 1.00284600 & 2.85628700 & 4.11382300 \\ \mathrm{H} & 1.38991900 & 1.29651000 & 4.09543000 \\ \mathrm{H} & 0.34674900 & 2.32531000 & -0.71596300 \\ \mathrm{H} & 1.62534100 & -1.10131000 & 0.11791200 \\ \mathrm{C} & 0.13539500 & 0.35764100 & -0.05523400 \\ \mathrm{C} & 0.86978400 & 1.41853600 & -0.42804800 \\ \mathrm{H} & -0.94740700 & 0.35305600 & -0.01565100 \\ \mathrm{H} & 1.95818500 & 1.40713300 & -0.43295700\end{array}$

MoN-EP2,$E=-5429.467476$

$\begin{array}{llcc}\mathrm{N} & -5.67002000 & 1.51314400 & -0.87514400 \\ \mathrm{C} & -5.22599900 & 2.50958500 & -1.90940600 \\ \mathrm{C} & -4.43559300 & 1.88501700 & -3.08326800 \\ \mathrm{O} & -4.52838700 & 2.31933600 & -4.24581300 \\ \mathrm{C} & -4.32253200 & 3.67063100 & -1.35500100 \\ \mathrm{C} & -2.94354400 & 3.16288200 & -0.87590800 \\ \mathrm{O} & -1.88718100 & 3.51010600 & -1.50927300 \\ \mathrm{O} & -2.99275900 & 2.36546800 & 0.16032200 \\ \mathrm{~N} & -0.54014800 & 7.87424300 & 1.42488800 \\ \mathrm{C} & -1.71664300 & 8.39779400 & 0.71878000 \\ \mathrm{C} & -2.96620500 & 7.54235800 & 0.99131000 \\ \mathrm{O} & -4.03515400 & 7.98697000 & 1.46586000 \\ \mathrm{C} & -1.43404200 & 8.55666700 & -0.80626300 \\ \mathrm{C} & -2.61212400 & 9.21885600 & -1.57026300 \\ \mathrm{C} & -1.03473400 & 7.22453800 & -1.47950800 \\ \mathrm{C} & -3.00512000 & 10.62856000 & -1.07492300 \\ & & & \mathrm{~S} 104\end{array}$




\begin{tabular}{|c|c|c|c|}
\hline N & 2.79555800 & 2.65597500 & 7.67938200 \\
\hline $\mathrm{C}$ & 3.89117900 & 1.71106500 & 7.93297000 \\
\hline $\mathrm{C}$ & 4.71972400 & 1.27796800 & 6.69969600 \\
\hline $\mathrm{O}$ & 5.77031500 & 0.57502400 & 6.85520300 \\
\hline $\mathrm{C}$ & 3.36673100 & 0.47839900 & 8.69876100 \\
\hline $\mathrm{N}$ & 4.32772300 & 1.71505400 & 5.46836500 \\
\hline $\mathrm{C}$ & 5.03170800 & 1.24417400 & 4.25679600 \\
\hline $\mathrm{C}$ & 6.53764300 & 1.16502000 & 4.50016100 \\
\hline $\mathrm{O}$ & 7.27140800 & 0.31178500 & 3.95836700 \\
\hline $\mathrm{C}$ & 4.71791100 & 2.17636400 & 3.04433700 \\
\hline $\mathrm{C}$ & 5.58981000 & 3.44029200 & 2.94808100 \\
\hline $\mathrm{S}$ & 5.02478900 & 4.62143400 & 1.62503700 \\
\hline $\mathrm{C}$ & 5.35820000 & 3.60614900 & 0.11534700 \\
\hline $\mathrm{N}$ & 5.65308400 & -2.23800100 & 3.36876400 \\
\hline $\mathrm{C}$ & 4.89773600 & -2.46583900 & 2.13171400 \\
\hline $\mathrm{C}$ & 5.35255800 & -1.43211300 & 1.06317600 \\
\hline $\mathrm{O}$ & 5.10255900 & -0.20034700 & 1.18210600 \\
\hline $\mathrm{C}$ & 3.36492600 & -2.36424400 & 2.31974100 \\
\hline $\mathrm{S}$ & 2.42256500 & -3.08018000 & 0.86825400 \\
\hline $\mathrm{N}$ & 6.05942400 & -1.90485300 & -0.01805900 \\
\hline $\mathrm{C}$ & 6.45423000 & -1.08188500 & -1.19417300 \\
\hline $\mathrm{C}$ & 7.42204300 & 0.04697000 & -0.80123100 \\
\hline $\mathrm{O}$ & 8.46583400 & 0.28996400 & -1.43804400 \\
\hline $\mathrm{C}$ & 5.22070500 & -0.51889000 & -1.99380900 \\
\hline $\mathrm{C}$ & 4.34018300 & -1.70232000 & -2.47390500 \\
\hline $\mathrm{C}$ & 5.69340900 & 0.36476100 & -3.17158700 \\
\hline $\mathrm{C}$ & 3.04613700 & -1.27419900 & -3.19626300 \\
\hline $\mathrm{N}$ & -8.99792300 & -5.04831900 & -3.65246400 \\
\hline $\mathrm{C}$ & -8.62154400 & -4.20914100 & -4.81907100 \\
\hline $\mathrm{C}$ & -9.19762700 & -4.86654300 & -6.08346100 \\
\hline $\mathrm{O}$ & -8.74860200 & -4.73640500 & -7.23623800 \\
\hline $\mathrm{C}$ & -7.11763200 & -3.88875100 & -5.01748100 \\
\hline $\mathrm{C}$ & -6.45518200 & -2.95405900 & -4.03085800 \\
\hline $\mathrm{C}$ & -6.96702500 & -1.91633500 & -3.25781700 \\
\hline $\mathrm{C}$ & -5.02106400 & -2.95020600 & -3.81407300 \\
\hline $\mathrm{N}$ & -5.93090200 & -1.27486000 & -2.56300000 \\
\hline $\mathrm{C}$ & -4.72549500 & -1.89712200 & -2.89243600 \\
\hline $\mathrm{C}$ & -3.96808600 & -3.75264100 & -4.31722500 \\
\hline $\mathrm{C}$ & -3.41250500 & -1.65162300 & -2.44194100 \\
\hline $\mathrm{C}$ & -2.66087900 & -3.50364500 & -3.88039500 \\
\hline $\mathrm{C}$ & -2.39103900 & -2.46858000 & -2.94346000 \\
\hline $\mathrm{N}$ & 3.26373100 & -5.57275600 & -7.77456300 \\
\hline $\mathrm{C}$ & 3.93074900 & -6.20907600 & -6.63054400 \\
\hline $\mathrm{C}$ & 2.96820500 & -6.96095800 & -5.69918700 \\
\hline $\mathrm{O}$ & 1.77971200 & -7.19303200 & -5.99555700 \\
\hline $\mathrm{C}$ & 4.79634100 & -5.16712800 & -5.85560500 \\
\hline $\mathrm{C}$ & 5.73236300 & -5.75572700 & -4.82680500 \\
\hline $\mathrm{C}$ & 6.97639200 & -6.31524400 & -5.07795400 \\
\hline $\mathrm{C}$ & 5.52634600 & -5.83398000 & -3.38835100 \\
\hline $\mathrm{N}$ & 7.55584400 & -6.74344600 & -3.87808200 \\
\hline
\end{tabular}




\begin{tabular}{|c|c|c|c|}
\hline $\mathrm{C}$ & 6.68658700 & -6.45239900 & -2.82231900 \\
\hline $\mathrm{C}$ & 4.48235700 & -5.41778600 & -2.52549100 \\
\hline $\mathrm{C}$ & 6.82829200 & -6.66296000 & -1.43706600 \\
\hline $\mathrm{C}$ & 4.61408900 & -5.61887100 & -1.14471400 \\
\hline $\mathrm{C}$ & 5.77828500 & -6.23723800 & -0.60789100 \\
\hline $\mathrm{N}$ & 2.46653100 & 8.65742000 & -4.64265500 \\
\hline $\mathrm{C}$ & 3.48483200 & 7.59607000 & -4.73282500 \\
\hline $\mathrm{C}$ & 4.52647500 & 8.21382000 & -5.67428200 \\
\hline $\mathrm{O}$ & 4.89426100 & 7.74315900 & -6.76750700 \\
\hline $\mathrm{C}$ & 3.01969800 & 6.16689700 & -5.16691700 \\
\hline $\mathrm{C}$ & 1.66468600 & 5.71083500 & -4.66485900 \\
\hline $\mathrm{C}$ & 1.33935000 & 5.04749300 & -3.48113000 \\
\hline $\mathrm{C}$ & 0.42871700 & 5.79945700 & -5.42190500 \\
\hline $\mathrm{N}$ & -0.01510700 & 4.71717300 & -3.46277400 \\
\hline $\mathrm{C}$ & -0.59708400 & 5.16566500 & -4.64354500 \\
\hline $\mathrm{C}$ & 0.09766300 & 6.33346800 & -6.69246500 \\
\hline $\mathrm{C}$ & -1.92273200 & 5.04431200 & -5.10662000 \\
\hline $\mathrm{C}$ & -1.22304100 & 6.22711500 & -7.15090900 \\
\hline $\mathrm{C}$ & -2.22254400 & 5.58597000 & -6.36496100 \\
\hline $\mathrm{N}$ & -10.11584500 & -2.10103700 & 5.87963000 \\
\hline $\mathrm{C}$ & -8.85124900 & -1.71332500 & 5.29539500 \\
\hline $\mathrm{C}$ & -7.86255900 & -1.59073900 & 6.42788600 \\
\hline $\mathrm{O}$ & -8.11645400 & -1.93861000 & 7.59964400 \\
\hline $\mathrm{C}$ & -8.95592900 & -0.38476800 & 4.47180000 \\
\hline $\mathrm{C}$ & -7.64200900 & 0.15723500 & 3.92104900 \\
\hline $\mathrm{C}$ & -6.78737400 & -0.63283000 & 3.11584500 \\
\hline $\mathrm{C}$ & -7.24907400 & 1.48319000 & 4.21091400 \\
\hline $\mathrm{C}$ & -5.57289300 & -0.11661600 & 2.62460100 \\
\hline $\mathrm{C}$ & -6.04293100 & 2.00866000 & 3.70743600 \\
\hline $\mathrm{C}$ & -5.19613300 & 1.21076300 & 2.91424700 \\
\hline $\mathrm{C}$ & 0.23406800 & -0.48024700 & 6.16375600 \\
\hline $\mathrm{O}$ & 0.44525700 & -1.16520500 & 7.43307000 \\
\hline $\mathrm{C}$ & 0.22277800 & -1.42034600 & 4.97738800 \\
\hline $\mathrm{S}$ & -0.09401000 & -0.71235400 & 3.39704100 \\
\hline $\mathrm{C}$ & 0.46314900 & -2.76075900 & 5.08254300 \\
\hline $\mathrm{S}$ & 0.49022700 & -3.80020000 & 3.65386600 \\
\hline $\mathrm{C}$ & 0.76350800 & -3.39455200 & 6.43267500 \\
\hline $\mathrm{C}$ & 1.33707100 & -2.32311300 & 7.36973800 \\
\hline $\mathrm{C}$ & -2.79874100 & -5.94625600 & 0.03709100 \\
\hline $\mathrm{O}$ & -4.25432900 & -5.97407700 & 0.19502900 \\
\hline $\mathrm{C}$ & -2.19393200 & -4.61416100 & 0.45164100 \\
\hline $\mathrm{S}$ & -0.45641000 & -4.57155600 & 0.77768000 \\
\hline $\mathrm{C}$ & -2.95864800 & -3.49362900 & 0.61265400 \\
\hline $\mathrm{S}$ & -2.20726500 & -1.96748200 & 1.10097800 \\
\hline $\mathrm{C}$ & -4.46998000 & -3.52684300 & 0.43617900 \\
\hline $\mathrm{C}$ & -4.88845000 & -4.77557600 & -0.35797700 \\
\hline Mo & 0.06704500 & -2.44393300 & 1.70737000 \\
\hline $\mathrm{O}$ & -1.53039200 & 2.10204000 & 2.20776000 \\
\hline $\mathrm{O}$ & 1.66676200 & 2.22069600 & 4.48889500 \\
\hline $\mathrm{O}$ & 1.06435800 & 5.46080200 & 1.42980200 \\
\hline
\end{tabular}




$\begin{array}{lrrr}\mathrm{O} & -0.08719400 & 3.81356700 & 3.23186600 \\ \mathrm{O} & 0.49953900 & -0.68998700 & 0.58088300 \\ \mathrm{H} & -6.87357900 & -1.18107700 & 6.16570700 \\ \mathrm{H} & -7.89018900 & 2.10566300 & 4.83370500 \\ \mathrm{H} & -5.76009400 & 3.03341600 & 3.93817500 \\ \mathrm{H} & -4.26064200 & 1.60973400 & 2.53194500 \\ \mathrm{H} & -4.90878500 & -0.74324200 & 2.03425000 \\ \mathrm{H} & -7.06476500 & -1.65691500 & 2.86892100 \\ \mathrm{H} & -9.65993500 & -0.58820800 & 3.65073300 \\ \mathrm{H} & -9.43667000 & 0.35914700 & 5.11798500 \\ \mathrm{H} & -10.06264900 & -2.43379600 & 6.83688800 \\ \mathrm{H} & -10.79063100 & -2.55966600 & 5.28222600 \\ \mathrm{H} & -8.41974100 & -2.48217600 & 4.62075200 \\ \mathrm{H} & 2.33725600 & -2.01741100 & 7.02062700 \\ \mathrm{H} & 1.41044600 & -2.67575900 & 8.40416400 \\ \mathrm{H} & -0.15189500 & -3.81957500 & 6.87001200 \\ \mathrm{H} & 1.48296600 & -4.21706800 & 6.31526300 \\ \mathrm{H} & -0.72772600 & 0.03556000 & 6.27139000 \\ \mathrm{H} & 1.01238600 & 0.28902000 & 6.03225300 \\ \mathrm{H} & -2.54255300 & -6.17312600 & -1.01336800 \\ \mathrm{H} & -2.42272800 & -6.75809700 & 0.66842300 \\ \mathrm{H} & -4.81884200 & -2.62731400 & -0.08665400 \\ \mathrm{H} & -4.94878000 & -3.53971300 & 1.42797000 \\ \mathrm{H} & -4.61230200 & -4.66213200 & -1.41738700 \\ \mathrm{H} & -1.49121000 & 6.62664400 & -8.12749600 \\ \mathrm{H} & -5.96617900 & -4.95847100 & -0.28360400 \\ \mathrm{H} & 3.39894100 & -7.33490300 & -4.75244300 \\ \mathrm{H} & 7.71706000 & -7.13080600 & -1.01868300 \\ \mathrm{H} & 5.85058200 & -6.38013700 & 0.46782900 \\ \mathrm{H} & 3.82872400 & -5.27995100 & -0.47257400 \\ \mathrm{H} & 3.58988000 & -4.93397500 & -2.91550500 \\ \mathrm{H} & 8.47000500 & -7.16112300 & -3.78524000 \\ \mathrm{H} & 7.49663300 & -6.42759600 & -6.01906700 \\ \mathrm{H} & 4.12893900 & -4.43420200 & -5.37966000 \\ \mathrm{H} & 5.36554100 & -4.62426600 & -6.62112900 \\ \mathrm{H} & 2.94717300 & -4.62501700 & -7.57145400 \\ \mathrm{H} & 2.49041400 & -6.13212600 & -8.13452100 \\ \mathrm{H} & 4.62753300 & -6.98057300 & -7.00306300 \\ \mathrm{H} & 4.90016200 & 9.19346400 & -5.31725700 \\ \mathrm{H} & 1.93313600 & 8.64085100 & -3.77636300 \\ \mathrm{H} & 3.95197900 & 8.68897300 & -5.44723300 \\ \mathrm{H} & -2360100 & 7.53931700 & -3.73968400 \\ \mathrm{H} & -1.31732300 & 2.47361500\end{array}$




\begin{tabular}{|c|c|c|c|}
\hline $\mathrm{H}$ & 3.08440300 & -2.93911100 & 3.21038900 \\
\hline $\mathrm{H}$ & 6.24025100 & -1.40935400 & 3.44480400 \\
\hline & 5.18662900 & -2.51240000 & 4.22555300 \\
\hline & 5.11223100 & -3.47562400 & 1.73841900 \\
\hline & 6.19544300 & -2.90747100 & -0.08830100 \\
\hline & 7.00822600 & -1.74775400 & -1.87030700 \\
\hline & 4.62759000 & 0.09182000 & -1.30028900 \\
\hline & 6.26898800 & 1.23575100 & -2.83680400 \\
\hline & 6.33101300 & -0.20587500 & -3.86249400 \\
\hline & 4.83243600 & 0.73886900 & -3.73641900 \\
\hline & 3.24045700 & -0.77314300 & -4.15326400 \\
\hline & 2.41325000 & -2.14906800 & -3.39750200 \\
\hline & 2.46314300 & -0.59046400 & -2.56618700 \\
\hline & 4.94016800 & -2.34636700 & -3.13937100 \\
\hline $\mathrm{H}$ & 4.05445300 & -2.30610200 & -1.60445000 \\
\hline & 7.12745400 & 0.64324800 & 0.07663500 \\
\hline $\mathrm{H}$ & -3.74700800 & 1.06056400 & -2.83036100 \\
\hline $\mathrm{H}$ & -6.51955700 & 1.82067400 & -0.40230500 \\
\hline 1 & -4.91792200 & 1.36887200 & -0.19037000 \\
\hline 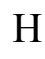 & -6.12186400 & 2.96666900 & -2.34934000 \\
\hline 1 & -4.17936800 & 4.41761900 & -2.14307600 \\
\hline $\mathrm{H}$ & -4.84166800 & 4.13482600 & -0.50643000 \\
\hline П & -10.09189400 & -5.48972700 & -5.87758000 \\
\hline $\mathrm{H}$ & -8.97685900 & -4.53558900 & -2.77329200 \\
\hline $\mathrm{H}$ & -8.43890000 & -5.89873300 & -3.57981900 \\
\hline $\mathrm{H}$ & -9.17764200 & -3.26143600 & -4.71370400 \\
\hline 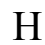 & -6.57206400 & -4.84576500 & -5.01553800 \\
\hline $\mathrm{H}$ & -7.00230100 & -3.49164800 & -6.03685600 \\
\hline $\mathrm{H}$ & -7.98666000 & -1.57031000 & -3.15437400 \\
\hline $\mathrm{H}$ & -6.01194500 & -0.44637900 & -1.96040800 \\
\hline $\mathrm{H}$ & -3.19567500 & -0.89938900 & -1.68903200 \\
\hline $\mathrm{H}$ & -1.37488200 & -2.33008300 & -2.58397500 \\
\hline $\mathrm{H}$ & -1.83965400 & -4.11692400 & -4.24396100 \\
\hline $\mathrm{H}$ & -4.16604900 & -4.55228400 & -5.02813300 \\
\hline $\mathrm{H}$ & 6.97405100 & 1.98495800 & 5.09693000 \\
\hline $\mathrm{H}$ & 2.51695200 & 0.02191600 & 8.17918000 \\
\hline $\mathrm{H}$ & 4.16384600 & -0.26407000 & 8.81080000 \\
\hline $\mathrm{H}$ & 3.01567900 & 0.79227200 & 9.68830700 \\
\hline $\mathrm{H}$ & 1.99213200 & 2.26730700 & 7.18921600 \\
\hline $\mathrm{H}$ & 3.07826300 & 3.55178800 & 7.29046000 \\
\hline $\mathrm{H}$ & 4.62553300 & 2.21298200 & 8.58049900 \\
\hline $\mathrm{H}$ & 3.38848400 & 2.11109600 & 5.31086800 \\
\hline $\mathrm{H}$ & 4.68842400 & 0.23669800 & 3.98773000 \\
\hline $\mathrm{H}$ & 4.84296100 & 1.56586100 & 2.15134600 \\
\hline $\mathrm{H}$ & 3.66256600 & 2.46371300 & 3.11154800 \\
\hline $\mathrm{H}$ & 5.54723200 & 4.03381800 & 3.87229700 \\
\hline $\mathrm{H}$ & 6.64186300 & 3.19916800 & 2.74805600 \\
\hline $\mathrm{H}$ & 5.07287400 & 4.21824700 & -0.74775800 \\
\hline $\mathrm{H}$ & 6.42500400 & 3.36353300 & 0.04716400 \\
\hline & 4.76821500 & 2.68543600 & 0.11905500 \\
\hline
\end{tabular}




$\begin{array}{lrrr}\mathrm{H} & -2.83978100 & 6.45596500 & 0.80601200 \\ \mathrm{H} & -0.54849700 & 7.99770500 & 2.43178300 \\ \mathrm{H} & -0.05945700 & 7.01512400 & 1.13529800 \\ \mathrm{H} & -1.94015500 & 9.39410500 & 1.12421600 \\ \mathrm{H} & -0.56701400 & 9.23588200 & -0.85513700 \\ \mathrm{H} & -1.86056300 & 6.50160300 & -1.49078000 \\ \mathrm{H} & -0.74722500 & 7.39840400 & -2.52427300 \\ \mathrm{H} & -0.18705100 & 6.74540100 & -0.97809800 \\ \mathrm{H} & -3.44961400 & 10.59319700 & -0.07297100 \\ \mathrm{H} & -2.12790200 & 11.29107900 & -1.03746600 \\ \mathrm{H} & -3.74355300 & 11.08542000 & -1.74877900 \\ \mathrm{H} & -2.32612000 & 9.27748100 & -2.63138100 \\ \mathrm{H} & -3.49474200 & 8.56220200 & -1.53003600 \\ \mathrm{H} & -2.05522000 & 2.17751400 & 1.32389500 \\ \mathrm{H} & -1.38708500 & 1.18687300 & 2.52530400 \\ \mathrm{H} & -0.60680100 & 4.38972800 & 3.82382900 \\ \mathrm{H} & -0.72272300 & 3.08749500 & 2.75776400 \\ \mathrm{H} & 0.65230300 & 4.79502200 & 2.05475600 \\ \mathrm{H} & 2.03360700 & 5.34300500 & 1.39072000 \\ \mathrm{H} & 1.07339100 & 2.91709800 & 4.06550600 \\ \mathrm{H} & 1.42694600 & 1.34802200 & 4.10487400 \\ \mathrm{H} & -0.18522200 & 1.95699200 & -0.85306900 \\ \mathrm{H} & 1.30034200 & 1.70658300 & 0.10929500 \\ \mathrm{C} & 0.33032300 & -0.09831700 & -0.51884200 \\ \mathrm{C} & 0.71596300 & 1.33209500 & -0.73548200 \\ \mathrm{H} & -0.15452200 & -0.64096000 & -1.34396400 \\ \mathrm{H} & 1.29004800 & 1.43139100 & -1.66818400\end{array}$

LL-ES, $E=-1159.326754$

$\begin{array}{lrrc}\mathrm{C} & 5.81771000 & 1.22187900 & 0.05325400 \\ \mathrm{C} & -2.48832200 & -2.56876700 & -1.69547000 \\ \mathrm{C} & -1.15902000 & -3.03473500 & 3.30779800 \\ \mathrm{C} & -3.94044200 & -2.07738600 & 2.80730400 \\ \mathrm{C} & -2.08484300 & 4.82363400 & -0.73627200 \\ \mathrm{C} & 0.29682900 & 4.66159100 & 1.02390600 \\ \mathrm{C} & 4.48728500 & 1.74073100 & 0.56692400 \\ \mathrm{O} & 4.22998600 & 1.97197000 & 1.78048400 \\ \mathrm{O} & 3.55137100 & 1.86387800 & -0.44353600 \\ \mathrm{~S} & -2.09683500 & -0.90370800 & -2.53098600 \\ \mathrm{C} & -1.48667700 & -2.01148100 & 2.26177000 \\ \mathrm{~S} & -0.07968000 & -1.31360200 & 1.30728500 \\ \mathrm{C} & -2.74905700 & -1.56659700 & 2.01944300 \\ \mathrm{~S} & -3.06809700 & -0.33271600 & 0.69216900 \\ \mathrm{C} & -1.33097900 & 3.54528800 & -0.53467000 \\ \mathrm{~S} & -2.17741100 & 2.05840300 & -1.20509400 \\ \mathrm{C} & -0.22129200 & 3.46848900 & 0.24397000 \\ \mathrm{~S} & 0.58328900 & 1.80989100 & 0.48297000 \\ \mathrm{~W} & -0.98565100 & 0.15370800 & -0.43347100 \\ \mathrm{O} & 2.63876400 & -0.17150200 & 2.63103100 \\ \mathrm{O} & 1.99945400 & -3.69137500 & 0.44443800 \\ & & & \mathrm{~S} 109\end{array}$




$\begin{array}{lrrr}\mathrm{O} & 2.98425100 & -0.70559200 & -1.37734600 \\ \mathrm{O} & 3.61538600 & -1.66683400 & 0.78813700 \\ \mathrm{O} & 0.54583500 & -0.56688600 & -1.64267500 \\ \mathrm{H} & -3.83823400 & -1.85097700 & 3.88819900 \\ \mathrm{H} & -4.87371500 & -1.60195700 & 2.45452700 \\ \mathrm{H} & -0.16712400 & -2.84184700 & 3.75989600 \\ \mathrm{H} & -1.12004000 & -4.06005200 & 2.88162300 \\ \mathrm{H} & -1.41629100 & 5.70631400 & -0.75519700 \\ \mathrm{H} & -2.65182500 & 4.80478400 & -1.68622000 \\ \mathrm{H} & 1.22150600 & 4.40904900 & 1.57486700 \\ \mathrm{H} & -0.45240600 & 5.00038900 & 1.76774000 \\ \mathrm{H} & -1.69291200 & -2.83240500 & -0.97844300 \\ \mathrm{H} & -3.44040000 & -2.46795000 & -1.15157100 \\ \mathrm{H} & 5.99646600 & 1.53774900 & -0.98670000 \\ \mathrm{H} & 5.78247900 & 0.11549500 & 0.08807200 \\ \mathrm{H} & 1.64984000 & -0.24452800 & 2.49110600 \\ \mathrm{H} & 2.95258200 & 0.77200900 & 2.57380700 \\ \mathrm{H} & 3.30189100 & -1.09724000 & 1.60518400 \\ \mathrm{H} & 3.15656700 & -2.58390400 & 0.78054700 \\ \mathrm{H} & 3.31076800 & -1.12506000 & -0.35995000 \\ \mathrm{H} & 3.35674000 & 0.20955900 & -1.48702700 \\ \mathrm{H} & 2.03592100 & -3.97500500 & -0.50539800 \\ \mathrm{H} & 1.17409800 & -3.14056100 & 0.61597500 \\ \mathrm{H} & 1.63843100 & -0.62462900 & -1.51034500 \\ \mathrm{H} & 0.12294400 & -0.96091300 & -2.47346300 \\ \mathrm{H} & 2.57215500 & 2.00863400 & -0.10025600 \\ \mathrm{C} & 2.81587200 & -4.56753200 & -2.95473500 \\ \mathrm{C} & 2.98201400 & -3.38135500 & -2.64729300 \\ \mathrm{H} & 2.67044400 & -5.59527500 & -3.23759600 \\ \mathrm{H} & 3.11563100 & -2.33002400 & -2.35173500 \\ \mathrm{H} & 0.52392800 & 5.52342200 & 0.36323700 \\ \mathrm{H} & -2.82633500 & 4.98906200 & 0.07450600 \\ \mathrm{H} & -2.56446500 & -3.34543300 & -2.48057200 \\ \mathrm{H} & -1.90568600 & -3.04605900 & 4.12412200 \\ \mathrm{H} & -4.06227700 & -3.17443900 & 2.70559900 \\ \mathrm{H} & 6.63240100 & 1.56330100 & 0.71099200\end{array}$

\begin{tabular}{lrrr}
\multicolumn{4}{l}{$\mathbf{L}-\mathbf{T S}, E=-1159.274040$} \\
$\mathrm{C}$ & 5.88197000 & 0.92095800 & 0.37562200 \\
$\mathrm{C}$ & -2.52762800 & -1.89135900 & -2.41485200 \\
$\mathrm{C}$ & -1.39162800 & -3.61313400 & 2.35607500 \\
$\mathrm{C}$ & -4.10690900 & -2.41675900 & 2.04103500 \\
$\mathrm{C}$ & -1.81298200 & 5.02330000 & 0.30675400 \\
$\mathrm{C}$ & 0.49759600 & 4.31728400 & 2.02710700 \\
$\mathrm{C}$ & 5.22602400 & 0.61804200 & -0.96950700 \\
$\mathrm{O}$ & 5.70227000 & 0.87653800 & -2.09816200 \\
$\mathrm{O}$ & 4.01261200 & -0.00931300 & -0.75677800 \\
$\mathrm{~S}$ & -2.00820800 & -0.10039200 & -2.79593600 \\
$\mathrm{C}$ & -1.65239700 & -2.37400400 & 1.55785300
\end{tabular}




$\begin{array}{lccc} & & & \\ \mathrm{S} & -0.18497200 & -1.51784000 & 0.87350100 \\ \mathrm{C} & -2.87716200 & -1.80334100 & 1.40621700 \\ \mathrm{~S} & -3.07504600 & -0.25935100 & 0.42629600 \\ \mathrm{C} & -1.13630600 & 3.68397200 & 0.22751500 \\ \mathrm{~S} & -2.00466800 & 2.44265900 & -0.80268500 \\ \mathrm{C} & -0.05436000 & 3.36894400 & 0.98795300 \\ \mathrm{~S} & 0.70083800 & 1.68787000 & 0.79919400 \\ \mathrm{~W} & -0.90128500 & 0.34422800 & -0.48083000 \\ \mathrm{O} & 2.26595400 & 0.63023000 & 3.19722700 \\ \mathrm{O} & 0.06916900 & -4.36299700 & -0.95343300 \\ \mathrm{O} & 3.57019400 & -2.11749700 & 0.52693300 \\ \mathrm{O} & 2.23229200 & -1.75141300 & 2.60358700 \\ \mathrm{O} & 0.63622600 & -0.22296500 & -1.75919400 \\ \mathrm{H} & -3.97622300 & -2.54626100 & 3.13356200 \\ \mathrm{H} & -4.99214200 & -1.77429500 & 1.88129300 \\ \mathrm{H} & -1.13292900 & -3.38343700 & 3.41277900 \\ \mathrm{H} & -0.54870400 & -4.19854000 & 1.93942200 \\ \mathrm{H} & -1.09982800 & 5.86161400 & 0.16695500 \\ \mathrm{H} & -2.59255300 & 5.11488900 & -0.47118200 \\ \mathrm{H} & 1.57105300 & 4.12776800 & 2.22384100 \\ \mathrm{H} & -0.04262100 & 4.21410600 & 2.99326500 \\ \mathrm{H} & -1.72067700 & -2.42692000 & -1.88700200 \\ \mathrm{H} & -3.42985700 & -1.86854000 & -1.78465900 \\ \mathrm{H} & 6.17440000 & -0.02599000 & 0.86695400 \\ \mathrm{H} & 5.16201400 & 1.43572400 & 1.03449000 \\ \mathrm{H} & 2.02769600 & 0.98759900 & 4.08119600 \\ \mathrm{H} & 1.73873600 & 1.11182900 & 2.43310000 \\ \mathrm{H} & 2.34241700 & -0.71921700 & 2.95629600 \\ \mathrm{H} & 1.27429200 & -1.81725800 & 2.19462800 \\ \mathrm{H} & 2.88304700 & -2.03545400 & 1.80689500 \\ \mathrm{H} & 3.80727500 & -1.13965000 & 0.25654700 \\ \mathrm{H} & 0.78504100 & -3.70468500 & -1.14947600 \\ \mathrm{H} & -0.64822200 & -3.85304900 & -0.49870800 \\ \mathrm{H} & 1.66972300 & -0.92037100 & -1.63452700 \\ \mathrm{H} & 0.20948300 & -0.34610700 & -2.67132700 \\ \mathrm{H} & 3.42900900 & -0.59069100 & -1.51587900 \\ \mathrm{C} & 2.57734000 & -1.95292800 & -1.70612800 \\ \mathrm{C} & 3.04706300 & -2.71993800 & -0.67304900 \\ \mathrm{H} & 2.34562800 & -2.52263700 & -2.62425000 \\ \mathrm{H} & 3.16235600 & -3.81020800 & -0.62454000 \\ \mathrm{H} & 0.40003800 & 5.37225400 & 1.70787100 \\ \mathrm{H} & -2.31160700 & 5.17678800 & 1.28701500 \\ \mathrm{H} & -2.73346900 & -2.40160000 & -3.37493400 \\ & -4.33968200 & -3.41307600 & 1.61163200 \\ \mathrm{H} & & & \\ \mathrm{H} & 6.77727400 & 1.54528700 & 0.22984900\end{array}$

LL-EP1, $E=-1159.318347$

$\begin{array}{llll}\text { C } & 5.82456100 & -1.17671600 & -0.84455500\end{array}$ 


\begin{tabular}{|c|c|c|c|}
\hline $\mathrm{C}$ & -3.38222400 & -0.95917400 & -2.11143700 \\
\hline $\mathrm{C}$ & -2.09541100 & -3.25728100 & 2.36958600 \\
\hline $\mathrm{C}$ & -4.28281200 & -1.23596700 & 2.55090500 \\
\hline $\mathrm{C}$ & -0.00479100 & 5.17134900 & 0.4847130 \\
\hline $\mathrm{C}$ & 2.19991000 & 3.65281000 & 1.76149600 \\
\hline $\mathrm{C}$ & 4.93826200 & 0.03755900 & -0.61505800 \\
\hline $\mathrm{O}$ & 5.31971800 & 1.21530800 & -0.45181800 \\
\hline $\mathrm{O}$ & 3.59100300 & -0.33827500 & -0.61607300 \\
\hline $\mathrm{S}$ & -2.41058600 & 0.59607300 & -2.5588370( \\
\hline $\mathrm{C}$ & -2.06407400 & -1.95114600 & 1.63942700 \\
\hline $\mathrm{S}$ & -0.55949100 & -1.61106900 & 0.65233200 \\
\hline $\mathrm{C}$ & -3.03863500 & -1.00531600 & 1.71449300 \\
\hline $\mathrm{S}$ & -2.86848800 & 0.59721500 & 0.82343100 \\
\hline $\mathrm{C}$ & 0.17607200 & 3.69473500 & 0.27384100 \\
\hline $\mathrm{S}$ & -1.21220100 & 2.88401500 & -0.63058300 \\
\hline $\mathrm{C}$ & 1.19609600 & 3.01582600 & 0.84813600 \\
\hline $\mathrm{S}$ & 1.29570800 & 1.16716800 & 0.63509500 \\
\hline W & -0.80994800 & 0.53976200 & -0.5153760 \\
\hline $\mathrm{O}$ & 2.24434400 & -0.22937900 & 3.16887500 \\
\hline $\mathrm{O}$ & -1.56324000 & -4.31278300 & -1.1128170 \\
\hline $\mathrm{O}$ & 3.11829300 & -2.80095700 & -0.05715800 \\
\hline $\mathrm{O}$ & 1.91627200 & -2.42298600 & 2.12492900 \\
\hline $\mathrm{O}$ & 0.31930900 & -0.14459300 & -1.98754600 \\
\hline $\mathrm{H}$ & -4.03278900 & -1.39822800 & 3.6187550 \\
\hline $\mathrm{H}$ & -4.95142500 & -0.35727700 & 2.4978300 \\
\hline $\mathrm{H}$ & -1.19285600 & -3.39024800 & 3.0030750 \\
\hline $\mathrm{H}$ & -2.11139600 & -4.11189500 & 1.6611380 \\
\hline $\mathrm{H}$ & 0.90791700 & 5.74860300 & 0.23457300 \\
\hline $\mathrm{H}$ & -0.82436600 & 5.55644200 & -0.14935600 \\
\hline $\mathrm{H}$ & 3.23226100 & 3.32336700 & 1.52780700 \\
\hline $\mathrm{H}$ & 2.01130200 & 3.38281200 & 2.82454800 \\
\hline $\mathrm{H}$ & -2.69004400 & -1.75400400 & -1.77805100 \\
\hline $\mathrm{H}$ & -4.07953600 & -0.72475300 & -1.2916760 \\
\hline $\mathrm{H}$ & 5.62629300 & -1.59611700 & -1.8475760 \\
\hline $\mathrm{H}$ & 5.59620900 & -1.96846600 & -0.1104550 \\
\hline $\mathrm{H}$ & 1.84923100 & 0.01392400 & 4.03616800 \\
\hline $\mathrm{H}$ & 1.91617500 & 0.40433100 & 2.40458800 \\
\hline $\mathrm{H}$ & 2.16240300 & -1.51145400 & 2.67951600 \\
\hline $\mathrm{H}$ & 0.95019200 & -2.27827800 & 1.72951400 \\
\hline $\mathrm{H}$ & 2.53539400 & -2.68122000 & 1.30647100 \\
\hline $\mathrm{H}$ & 3.21924500 & -1.82883900 & -0.4050940 \\
\hline $\mathrm{H}$ & -0.95139900 & -3.63128500 & -0.7151010 \\
\hline $\mathrm{H}$ & -2.36674300 & -3.82100900 & -1.4147510 \\
\hline $\mathrm{H}$ & 1.33507200 & -2.13702900 & -2.05487700 \\
\hline $\mathrm{H}$ & -0.18894600 & -0.12135700 & -2.8567700 \\
\hline $\mathrm{H}$ & 2.88863800 & 0.40433300 & -0.3832350 \\
\hline $\mathrm{C}$ & 1.58698000 & -3.20330100 & -1.93407400 \\
\hline $\mathrm{C}$ & 2.42510500 & -3.65220700 & -0.9713360 \\
\hline $\mathrm{H}$ & 1.08323300 & -3.93134100 & -2.5775860 \\
\hline $\mathrm{H}$ & 2.64630300 & -4.70552200 & -0.7698360 \\
\hline
\end{tabular}




$\begin{array}{rrrr}\mathrm{H} & 2.17537700 & 4.75446000 & 1.69308900 \\ \mathrm{H} & -0.27027500 & 5.40726900 & 1.53608800 \\ \mathrm{H} & -3.94051300 & -1.30253300 & -3.00499900 \\ \mathrm{H} & -2.97846100 & -3.34453600 & 3.02664400 \\ \mathrm{H} & -4.86092500 & -2.11500800 & 2.20247900 \\ \mathrm{H} & 6.88165400 & -0.87967600 & -0.77208700\end{array}$

LL-EP2, $E=-1159.345187$

$\begin{array}{lrrc}\mathrm{C} & 5.89706700 & -0.14781600 & -0.85952400 \\ \mathrm{C} & -3.22091100 & -1.60345200 & -1.93702900 \\ \mathrm{C} & -1.49603000 & -3.40203100 & 2.62440300 \\ \mathrm{C} & -4.00086800 & -1.78557900 & 2.75164000 \\ \mathrm{C} & -0.93584700 & 5.15005400 & 0.27635300 \\ \mathrm{C} & 1.51628000 & 4.10817500 & 1.57958200 \\ \mathrm{C} & 4.76480600 & 0.53450300 & -0.12683400 \\ \mathrm{O} & 4.81442600 & 0.76680500 & 1.12544900 \\ \mathrm{O} & 3.69952400 & 0.80689700 & -0.92438500 \\ \mathrm{~S} & -2.56221500 & 0.08485400 & -2.49617900 \\ \mathrm{C} & -1.69946700 & -2.14469500 & 1.83930900 \\ \mathrm{~S} & -0.29232200 & -1.59635700 & 0.80594600 \\ \mathrm{C} & -2.82776000 & -1.38774600 & 1.87941500 \\ \mathrm{~S} & -2.97440300 & 0.14541500 & 0.87299100 \\ \mathrm{C} & -0.49333400 & 3.72245000 & 0.12151700 \\ \mathrm{~S} & -1.73469600 & 2.63250900 & -0.69037800 \\ \mathrm{C} & 0.64056400 & 3.25437500 & 0.69921400 \\ \mathrm{~S} & 1.04290700 & 1.44247100 & 0.55612700 \\ \mathrm{~W} & -0.94992700 & 0.38866000 & -0.47010800 \\ \mathrm{O} & 2.87536400 & -0.02834900 & 2.63668600 \\ \mathrm{O} & -0.71636800 & -4.37305400 & -0.93559200 \\ \mathrm{O} & 3.81561400 & -2.58810600 & -0.46816100 \\ \mathrm{O} & 2.53895300 & -2.25374100 & 1.70174500 \\ \mathrm{O} & 0.22589700 & -0.29710300 & -1.93662000 \\ \mathrm{H} & -3.71373800 & -1.83655000 & 3.82074700 \\ \mathrm{H} & -4.81722800 & -1.04540900 & 2.66391000 \\ \mathrm{H} & -0.56996400 & -3.35291900 & 3.23369000 \\ \mathrm{H} & -1.39088000 & -4.28346200 & 1.95789700 \\ \mathrm{H} & -0.12108000 & 5.87048300 & 0.06199200 \\ \mathrm{H} & -1.77176800 & 5.37732800 & -0.41033900 \\ \mathrm{H} & 2.58227600 & 3.81539600 & 1.50717700 \\ \mathrm{H} & 1.22762500 & 4.00821200 & 2.64800800 \\ \mathrm{H} & -2.40953700 & -2.19880300 & -1.48225000 \\ \mathrm{H} & -4.00661800 & -1.44776600 & -1.18136400 \\ \mathrm{H} & 5.74675100 & -1.23785600 & -0.73889500 \\ \mathrm{H} & 6.86085300 & 0.12757800 & -0.40201800 \\ \mathrm{H} & 3.68973300 & 0.41392700 & 2.20221900 \\ \mathrm{H} & 2.06437300 & 0.50421400 & 2.34827600 \\ \mathrm{H} & 2.76507100 & -1.24076700 & 2.21288100 \\ \mathrm{H} & 1.53252500 & -2.27256800 & 1.51808600 \\ \mathrm{H} & 3.07344300 & -2.48992800 & 0.82761100 \\ \mathrm{H} & 1.91114500 & -2.79694100 & -3.19192800 \\ & & & \mathrm{~s} \\ & & & \end{array}$




$\begin{array}{lrrr}\mathrm{H} & -0.58505500 & -3.52301600 & -0.41917600 \\ \mathrm{H} & -1.39600500 & -4.19225500 & -1.62975600 \\ \mathrm{H} & 1.40482200 & -1.57295400 & -1.97248400 \\ \mathrm{H} & -0.32515200 & -0.39726600 & -2.77693100 \\ \mathrm{H} & 2.82902300 & 1.14004400 & -0.44431200 \\ \mathrm{C} & 1.96735700 & -2.57843800 & -2.10960100 \\ \mathrm{C} & 3.36740900 & -2.45180800 & -1.67167300 \\ \mathrm{H} & 1.37919800 & -3.31953200 & -1.53767700 \\ \mathrm{H} & 4.11454400 & -2.15794900 & -2.44340400 \\ \mathrm{H} & 1.44286800 & 5.17857900 & 1.31392600 \\ \mathrm{H} & -1.29667500 & 5.35561900 & 1.30570800 \\ \mathrm{H} & -3.63141000 & -2.13364600 & -2.81918400 \\ \mathrm{H} & -2.33620700 & -3.60027300 & 3.31250700 \\ \mathrm{H} & -4.41547600 & -2.77262000 & 2.46486700 \\ \mathrm{H} & 5.88589700 & 0.10325700 & -1.93147000\end{array}$

